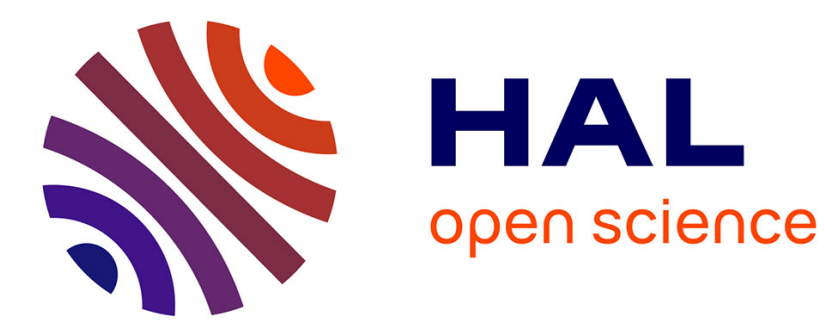

\title{
Simulation of cold spray particle deposition process
} Jing Xie

\section{- To cite this version:}

Jing Xie. Simulation of cold spray particle deposition process. Mechanics of materials [physics.classph]. INSA de Lyon, 2014. English. NNT: 2014ISAL0044 . tel-01153225

\section{HAL Id: tel-01153225 \\ https://theses.hal.science/tel-01153225}

Submitted on 19 May 2015

HAL is a multi-disciplinary open access archive for the deposit and dissemination of scientific research documents, whether they are published or not. The documents may come from teaching and research institutions in France or abroad, or from public or private research centers.
L'archive ouverte pluridisciplinaire HAL, est destinée au dépôt et à la diffusion de documents scientifiques de niveau recherche, publiés ou non, émanant des établissements d'enseignement et de recherche français ou étrangers, des laboratoires publics ou privés. 


\title{
THÈSE
}

\section{SIMULATION OF COLD SPRAY PARTICLE DEPOSITION PROCESS}

\author{
Présentée devant \\ l'Institut National des Sciences Appliquées de Lyon \\ pour obtenir \\ le GRADE DE DOCTEUR
}

École doctorale :

Mécanique, Énergétique, Génie Civil, Acoustique

Spécialité :

MÉCANIQUE - GÉNIE MÉCANIQUE - GÉNIE CIVIL

par

\section{Jing XIE}

thèse soutenue le 27 Mai 2014 devant la Commission d'examen

Jury

VINCENT GUIPONT

GHISLAIN MONTAVON

PHILIPPE BERTRAND

ALAIN COMBESCURE

BERNARD NORMAND

KAZUHIRO OGAWA

DANIEL NÉLIAS

HÉLÈNE WALTER-LE BERRE

YUJI ICHIKAWA

STÉPHANE KNITTEL

VINCENT ROBIN

ENSMP, Paris
UTBM, Belfort
ENISE, Saint-Etienne
INSA, Lyon
INSA, Lyon
Tohoku Univ., Sendai
INSA, Lyon
INSA, Lyon
Tohoku Univ., Sendai
SNECMA, Corbeil
AREVA NP, Lyon

Chercheur (HDR)

Professeur

Maître de Conférences (HDR)

Professeur

Professeur

Professeur

Professeur

Maître de Conférences

Professeur Adjoint

Ingénieur de Recherche

Expert
Rapporteur

Rapporteur

Examinateur

Examinateur

Examinateur

Examinateur

Directeur de thèse

Co-encadrant

Invité

Invité

Invité 
Cette thèse est accessible à l'adresse : http://theses.insa-lyon.fr/publication/2014ISAL0044/these.pdf (C) [J. Xie], [2014], INSA de Lyon, tous droits réservés 
INS4 Direction de la Recherche - Ecoles Doctorales - Quinquennal 2011-2015

\begin{tabular}{|c|c|c|}
\hline SIGLE & ECOLE DOCTORALE & NOM ET COORDONNEES DU RESPONSABLE \\
\hline CHIMIE & $\begin{array}{l}\text { CHIMIE DE LYON } \\
\text { http://www.edchimie-lyon.fr } \\
\text { Sec : Renée EL MELHEM } \\
\text { Bat Blaise Pascal } 3^{e} \text { etage } \\
0472438046 \\
\text { Insa : R. GOURDON }\end{array}$ & $\begin{array}{l}\text { M. Jean Marc LANCELIN } \\
\text { Université de Lyon - Collège Doctoral } \\
\text { Bât ESCPE } \\
43 \text { bd du } 11 \text { novembre } 1918 \\
69622 \text { VILLEURBANNE Cedex } \\
\text { Tél : 04.72.43 13 95 } \\
\text { directeur@edchimie-lyon.fr }\end{array}$ \\
\hline E.E.A. & \begin{tabular}{|l|} 
ELECTRONIQUE, \\
ELECTROTECHNIQUE, AUTOMATIQUE \\
http://edeea.ec-lyon.fr \\
Sec : M.C. HAVGOUDOUKIAN \\
eea@ec-lyon.fr
\end{tabular} & $\begin{array}{l}\text { M. Gérard SCORLETTI } \\
\text { Ecole Centrale de Lyon } \\
36 \text { avenue Guy de Collongue } \\
69134 \text { ECULLY } \\
\text { Tél : 04.72.1860.97 Fax : } 0478433717 \\
\text { Gerard.scorletti@ec-lyon.fr }\end{array}$ \\
\hline E2M2 & $\begin{array}{l}\text { EVOLUTION, ECOSYSTEME, } \\
\text { MICROBIOLOGIE, MODELISATION } \\
\text { http://e2m2.universite-lyon.fr } \\
\text { Sec : Safia AIT CHALAL } \\
\text { Bat Darwin - UCB Lyon } 1 \\
\text { 04.72.43.28.91 } \\
\text { Insa : H. CHARLES }\end{array}$ & $\begin{array}{l}\text { Mme Gudrun BORNETTE } \\
\text { CNRS UMR 5023 LEHNA } \\
\text { Université Claude Bernard Lyon } 1 \\
\text { Bât Forel } \\
43 \text { bd du } 11 \text { novembre } 1918 \\
69622 \text { VILLEURBANNE Cédex } \\
\text { Té1: 06.07.53.89.13 } \\
\text { e2m2@, univ-lyon1.fr }\end{array}$ \\
\hline EDISS & $\begin{array}{l}\text { INTERDISCIPLINAIRE SCIENCES- } \\
\text { SANTE } \\
\text { http://www.ediss-lyon.fr } \\
\text { Sec: Safia AIT CHALAL } \\
\text { Hôpital Louis Pradel - Bron } \\
0472684909 \\
\text { Insa: M. LAGARDE } \\
\text { Safia.ait-chalal@univ-lyon1.fr }\end{array}$ & $\begin{array}{l}\text { Mme Emmanuelle CANET-SOULAS } \\
\text { INSERM U1060, CarMeN lab, Univ. Lyon } 1 \\
\text { Bâtiment IMBL } \\
11 \text { avenue Jean Capelle INSA de Lyon } \\
696621 \text { Villeurbanne } \\
\text { Tél : 04.72.68.49.09 Fax :04 } 72684916 \\
\underline{\text { Emmanuelle.canet@univ-lyon } 1 . f r}\end{array}$ \\
\hline INFOMATHS & $\begin{array}{l}\text { INFORMATIQUE ET } \\
\text { MATHEMATIQUES } \\
\text { http://infomaths.univ-lyon1.fr } \\
\text { Sec :Renée EL MELHEM } \\
\text { Bat Blaise Pascal } \\
3^{e} \text { etage } \\
\text { infomaths@univ-lyon1.fr }\end{array}$ & $\begin{array}{l}\text { Mme Sylvie CALABRETTO } \\
\text { LIRIS - INSA de Lyon } \\
\text { Bat Blaise Pascal } \\
7 \text { avenue Jean Capelle } \\
69622 \text { VILLEURBANNE Cedex } \\
\text { Tél : 04.72. 43. 80. 46 Fax 04 } 72431687 \\
\text { Sylvie.calabretto@insa-lyon.fr }\end{array}$ \\
\hline Matériaux & $\begin{array}{l}\text { MATERIAUX DE LYON } \\
\text { http://ed34.universite-lyon.fr } \\
\text { Sec : M. LABOUNE } \\
\text { PM : } 71.70 \text {-Fax : } 87.12 \\
\text { Bat. Saint Exupéry } \\
\text { Ed.materiaux@insa-lyon.fr }\end{array}$ & $\begin{array}{l}\text { M. Jean-Yves BUFFIERE } \\
\text { INSA de Lyon } \\
\text { MATEIS } \\
\text { Bâtiment Saint Exupéry } \\
7 \text { avenue Jean Capelle } \\
\text { 69621 VILLEURBANNE Cedex } \\
\text { Tél : 04.72.43 83 18 Fax 04 } 72438528 \\
\text { Jean-yves.buffiere@insa-lyon.fr } \\
\end{array}$ \\
\hline MEGA & $\begin{array}{l}\text { MECANIQUE, ENERGETIQUE, GENIE } \\
\text { CIVIL, ACOUSTIQUE } \\
\text { http://mega.universite-lyon.fr } \\
\text { Sec : M. LABOUNE } \\
\text { PM : 71.70-Fax : } 87.12 \\
\text { Bat. Saint Exupéry } \\
\text { mega@insa-lyon.fr }\end{array}$ & $\begin{array}{l}\text { M. Philippe BOISSE } \\
\text { INSA de Lyon } \\
\text { Laboratoire LAMCOS } \\
\text { Bâtiment Jacquard } \\
25 \text { bis avenue Jean Capelle } \\
69621 \text { VILLEURBANNE Cedex } \\
\text { Tél:04.72.43.71.70 Fax : } 0472437237 \\
\text { Philippe.boisse@insa-lyon.fr }\end{array}$ \\
\hline ScSo & \begin{tabular}{|l}
$\frac{\text { ScSo* }}{\text { http: } / / \text { recherche.univ-lyon2.fr/scso/ }}$ \\
Sec : Viviane POLSINELLI \\
$\quad$ Brigitte DUBOIS \\
Insa : J.Y. TOUSSAINT
\end{tabular} & $\begin{array}{l}\text { Mme Isabelle VON BUELTZINGLOEWEN } \\
\text { Université Lyon } 2 \\
86 \text { rue Pasteur } \\
69365 \text { LYON Cedex } 07 \\
\text { Tél : 04.78.77.23.86 Fax : 04.37.28.04.48 } \\
\text { viviane.polsinelli@univ-lyon2.fr }\end{array}$ \\
\hline
\end{tabular}


Cette thèse est accessible à l'adresse : http://theses.insa-lyon.fr/publication/2014ISAL0044/these.pdf (C) [J. Xie], [2014], INSA de Lyon, tous droits réservés 


\section{All the art of living lies in a fine mingling of letting go and holding on.}

- Henry Ellis 
Cette thèse est accessible à l'adresse : http://theses.insa-lyon.fr/publication/2014ISAL0044/these.pdf (C) [J. Xie], [2014], INSA de Lyon, tous droits réservés 


\section{Abstract}

Cold spray is a rapidly developing coating technology for depositing materials in the solid state. The cold spray particle deposition process was simulated by modeling the high velocity impacts of spherical particles onto a flat substrate under various conditions. We, for the first time, proposed the Couple Eulerian Lagrangian (CEL) numerical approach to solve the high strain rate deformation problem. The capability of the CEL numerical approach in modeling the Cold Spray deposition process was verified through a systematic parameter study, including impact velocity, initial particle temperature, friction coefficient and materials combination. The simulation results by using the CEL numerical approach agree with the experimental results published in the literature. Comparing with other numerical approaches, which are Lagrangian, ALE and SPH, the CEL analyses are generally more accurate and more robust in higher deformation regimes.

Besides simulating the single particle impact problem, we also extended our study into the simulation of multiple impacts. A FCC-like particles arrangement model that inspired by the crystal structure was built to investigate the porosity rate and residual stress of deposited particles under various conditions. We observed not only the 3D profiles of voids, but also their distributions and developments during different procedures. Higher impact velocity and higher initial temperature of particles are both of benefit to produce a denser cold spray coating. The compressive residual stresses existed in the interface between the particle and substrate is mainly caused by the large and fast plastic deformation. Another simplified model for multiple impacts was created for the simulation of surface erosion. A severe surface erosion is the result of a high impact velocity, a high friction coefficient and a low contact angle.

Two element failure models suitable for high-strain-rate dynamic problems were introduced in this study. One is shear failure model driven by Johnson-Cook plastic yielding; another one is called tensile failure model, which the material fails when the mean stress reaches the ultimate tensile strength. For a ductile material as Copper, it followed two fracture modes in our study, which are tensile failure mode and shear failure mode. The former one mainly occurred beneath the substrate surface and the periphery of substrate craters, nevertheless the latter one was found predominately at the surface of craters. Four steps were found during the propagation of crack : void formation; crack formation; crack growth ; coalescence and failure. A simple criterion equation was derived based on the simulation results for predicting the initiation of damage, which the erosion velocity $v_{\text {ero }}$ is a function of contact angle $\theta$ and erosion velocity for normal impact $v_{\pi / 2}$. The equivalent plastic strain could also be a parameter for identifying the onset of damage, identified as being 1.042 for Copper in our study.

KEY WORDS: Cold Spray, CEL, multiple impacts, Porosity rate, Residual stress, Erosion rate, Fracture 
Cette thèse est accessible à l'adresse : http://theses.insa-lyon.fr/publication/2014ISAL0044/these.pdf (C) [J. Xie], [2014], INSA de Lyon, tous droits réservés 


\section{Résumé}

La projection à froid est une technologie en plein essor pour le dépôt de matériaux à l'état solide. Le procédé de dépôt des particules par pulvérisation à froid est simulé par la modélisation de l'impact à haute vitesse de particules sphériques sur un substrat plat dans diverses conditions. Pour la première fois, nous proposons une approche numérique par couplage Euler-Lagrange (CEL) afin de résoudre ce problème à haute vitesse de déformation. Les capacités de l'approache numérique CEL pour la modélisation du processus de dépôt de projection à froid sont évaluées par une étude paramétrique de : la vitesse d'impact, la température initiale des particules, le coefficient de frottement et le choix des matériaux. Les résultats de la simulation à l'aide de l'approche numérique CEL sont en accord avec les résultats expérimentaux publiés dans la littérature. La méthode CEL est généralement plus précise et plus robuste dans des régimes de déformations élevées que d'autres méthodes numériques de type Lagrangienne, ALE ou SPH.

Outre la simulation du problème de l'impact unique, nous étendrons également notre étude à la simulation des impacts multiples. Un nouveau modèle d'empilement de type CFC, inspiré de la structure cristalline, est construit afin d'étudier le taux de porosité des particules déposées et les contraintes résiduelles dans le matériau de substrat pour diverses conditions. Nous pouvons observer non seulement la goémétrie 3D de porosités, mais aussi leur répartition et leur évolution pendant les impacts successifs. Pour les particules, une vitesse d'impact et une température initiale élevées, sont des avantages pour produire des revêtements denses par projection à froid. Des contraintes résiduelles de compression existent à l'interface entre les particules et le substrat. Ces dernières sont causées par les grandes amplitudes et vitesses de déformation plastique induites par le procédé. Un second modèle moins complexe pour la modélisation de l'impact multiple oblique a été créé afin de simuler l'érosion de surface. Une forte érosion de surface est le résultat : d'une plus grande vitesse d'impact, d'un coefficient de frottement élevé et d'un angle de contact réduit.

Deux modèles d'endommagement particulièrement adaptés pour les problèmes à forte vitesse de déformation sont introduits dans cette étude. L'un est le modèle de rupture par cisaillement plastique de Johnson-Cook (mode 2); l'autre est un modèle d'endommagement en traction (mode 1), où le matériau disparaît lorsque la contrainte moyenne atteint la striction. Pour un matériau ductile comme le cuivre, il y a deux modes de rupture : le mode 1 de traction et le mode 2 de rupture par cisaillement. Le premier survient principalement en dessous de la surface du substrat et à la périphérie de impacts, tandis que le second intervient de manière prédominante à la surface des impacts. On observe quatre étapes lors de la propagation des fissures : la formation de porosités, de fissures, la croissance de ces dernières, puis une dernière étape de coalescence et rupture. Un critère simple, où la vitesse d'érosion $v_{\text {ero }}$ est fonction de l'angle de contact $\theta$ et de la vitesse critique d'érosion lors d'un impact de vitesse normale $v_{\pi / 2}$, est proposé sur la base des résultats des simulations afin de prédire l'initiation de l'endommagement. La déformation plastique équivalente est également un paramètre clef pour identifier l'initiation de 
l'endommagement, une valeur critique de 1,042 a été trouvée dans notre étude pour le cuivre.

MOTS CLÉS: Cold Spray/projection à froid, CEL, impacts multiples, taux de porosité, contraintes résiduelles, taux d'érosion, rupture 


\section{Contents}

$\begin{array}{ll}\text { Contents } & \text { i }\end{array}$

List of Figures $\quad$ v

List of Tables $\quad$ ix

$\begin{array}{ll}\text { Introduction } & 1\end{array}$

1 Previous researches on the cold spray deposition process 3

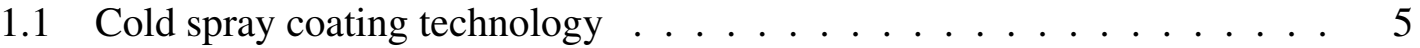

1.1.1 Coating formation process ............. 5

1.1.2 Advantages and disadvantages ................. 5

1.1.3 Bonding mechanism . . . . . . . . . . . . 6

1.1.4 Application ................... 8

1.2 Numerical approach . . . . . . . . . . . . . . . . . 9

1.2.1 Lagrangian . . . . . . . . . . . . . . . . 9

1.2.2 Arbitrary Lagrangian Eulerian . . . . . . . . . . . . . . . . 9

1.2.3 Smoothed Particle Hydrodynamics . . . . . . . . . . . . . . . . . 10

1.2.4 Coupled Eulerian Lagrangian . . . . . . . . . . . . . . . . 10

1.3 Previous studies . . . . . . . . . . . . . . . . 11

1.3.1 Simulation of cold spray . . . . . . . . . . . . . 11

1.3.2 Effect of parameters ........................... 14

1.3.3 Experimental results . . . . . . . . . . . . . . 17

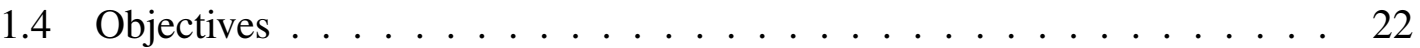

2 Feasibility of numerical approach $\quad \mathbf{2 5}$

2.1 Problem description . . . . . . . . . . . . . . . . . . . . . 27

2.2 Basic assumptions . . . . . . . . . . . . . . . . 27

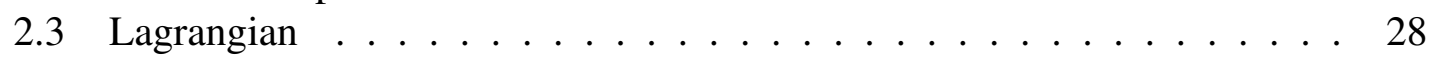

2.3.1 Model description . . . . . . . . . . . . . . . 28

2.3.2 Results and discussion . . . . . . . . . . . . . . 34

2.4 Arbitrary Lagrangian Eulerian . . . . . . . . . . . . . . . 36

2.4 .1 Model description . . . . . . . . . . . . . . 36

2.4 .2 Results and discussion . . . . . . . . . . . . 38 
2.5 Smoothed Particle Hydrodynamics . . . . . . . . . . . . . . . . 40

2.5.1 Model description . . . . . . . . . . . . . . . . . 44 40

2.5.2 Results and discussion . . . . . . . . . . . . . . . 43

2.6 Coupled Eulerian Lagrangian . . . . . . . . . . . . . . . . . . . 44

2.6.1 Model description . . . . . . . . . . . . . . . . . 44

2.6.2 Results and discussion . . . . . . . . . . . . 47

2.7 Discussion . . . . . . . . . . . . . . . . . . . . . 48

2.7.1 Comparison of four numerical approaches . . . . . . . . . . . 48

2.7.2 Typical impact behaviors of cold sprayed particle and substrate . . 50

2.8 Summary . . . . . . . . . . . . . . . . . . . . . 54

3 Simulation of single impact

3.1 Simulation outline . . . . . . . . . . . . . . . . 57

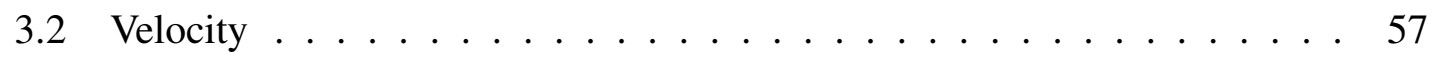

3.2 .1 Deformation shape ....................... 57

3.2.2 Evolution of the strain and temperature .......... . . 59

3.3 Temperature . . . . . . . . . . . . . . . . 60

3.3.1 Deformation shape .................... 60

3.3.2 Evolution of the strain and temperature .......... 61

3.4 Friction coefficient . . . . . . . . . . . . . . 63

3.4 .1 Deformation shape . . . . . . . . . . . . 63

3.4.2 Evolution of the strain and temperature . . . . . . . . 63

3.5 Material . . . . . . . . . . . . . . . . . . 64

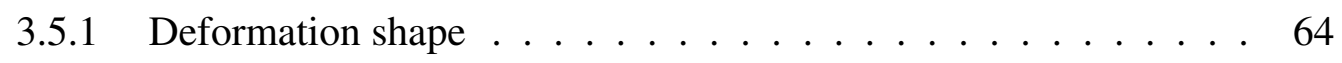

3.5.2 Evolution of the strain and temperature . . . . . . . . 65

3.6 Discussion . . . . . . . . . . . . . . . . 66

3.6.1 Energy dissipation and restitution coefficient ........ 66

3.7 Summary . . . . . . . . . . . . . . . . . . 69

4 Simulation of multiple impacts $\quad 71$

4.1 Simulation outline . . . . . . . . . . . . . . . . 72

4.2 Deformation shape ..................... 73

4.3 Porosity rate . . . . . . . . . . . . . . . . . . . . . . . . . . . . . . . 74

4.4 Residual stress . . . . . . . . . . . . . . . . . 77

4.5 Summary . . . . . . . . . . . . . . . . . 79

5 Simulation of erosion $\quad 81$

5.1 Simulation outline . . . . . . . . . . . . . . . . 83

5.1.1 Dynamic failure model . . . . . . . . . . . . . 83

5.2 Oblique impact . . . . . . . . . . . . . . . . 85

5.2 .1 Deformation shape . . . . . . . . . . . . 85

5.2.2 Evolution of the strain and temperature . . . . . . . . 86

5.3 Surface erosion . . . . . . . . . . . . . . . . 87

ii 
5.3.1 Contact angle . . . . . . . . . . . . . . 88

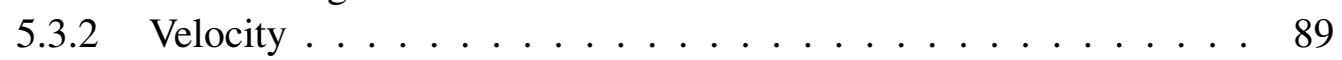

5.3 .3 Friction coefficient . . . . . . . . . . . . . 990

5.3 .4 Multiple impacts . . . . . . . . . . . . . . . . 91

5.4 Discussion . . . . . . . . . . . . . . . . . . 91

5.4 .1 Fracture ........................... 91

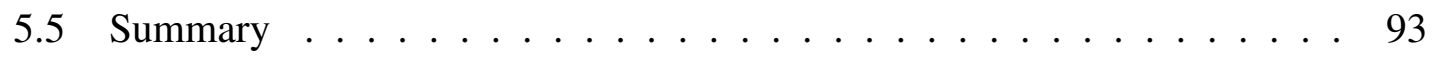

$\begin{array}{lr}\text { Conclusions } & 95\end{array}$

Appendix A: Significance of the substrate size in simulations of the normal im$\begin{array}{ll}\text { pact of a sphere with a half-space. } & 99\end{array}$

Appendix B: The temperature-dependent material property for Aluminum and Copper

Appendix C: Comparison of Analysis Procedures 105

$\begin{array}{ll}\text { Appendix D: Contact pair algorithm in ABAQUS/Explicit } & 107\end{array}$

Appendix E: ALE adaptive mesh controls

$\begin{array}{ll}\text { Appendix F: Finite element conversion to SPH particles } & 113\end{array}$

Appendix G: Assigning materials to Eulerian part 121

$\begin{array}{lr}\text { Appendix H: Modeling surface erosion } & 123\end{array}$

$\begin{array}{ll}\text { Appendix I: Progressive damage and failure } & 125\end{array}$

$\begin{array}{ll}\text { Appendix J: Damage initiation criterion } & 131\end{array}$

$\begin{array}{ll}\text { Bibliography } & 135\end{array}$ 


\section{List of Figures}

1.1 The schematic diagram of cold spray apparatus. . . . . . . . . . . 5

1.2 The stages of coating formation in the cold spray deposition process [STE 02]. 5

1.3 Microstructural changes across the interfacial zone. (a) Dendrites inside the intermetallic inclusion. Microstructures of the base plates in areas directly adhering to the intermetallic inclusion: (b) elongated subgrains inside the aluminum sheet and (c) ?ne grains inside copper sheet. TEM bright ?eld images in the ND-TD section [HEN 13] . . . . . . . . . . 6

1.4 The structures of the cross sections of the samples for three different values of Detonation velocity (D) and at the same thickness of explosive charge ? $5 \mathrm{~mm}$ : (a) $\mathrm{D}=3.19 \mathrm{~km} / \mathrm{s}$; (b) $\mathrm{D}=3.95 \mathrm{~km} / \mathrm{s}$; (c) $\mathrm{D}=5.26 \mathrm{~km} / \mathrm{s}$

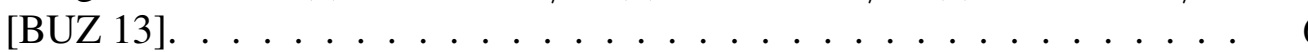

1.5 (a) Typical stress-strain curves in a normal strainhardening material ('Isothermal'), an adiabatically softened material ('Adiabatic') and in a material undergoing an adiabatic shear localization ('Localization'); (b) schematics of the uniform and the localized simple shears [CHA 05] . . . . . . . 8

1.6 (a) Low-magnification TEM image of interfacial microstructures between as-sprayed Ni coatings and Al-6061-T6 alloy substrate in a kinetic spray process. HREM images of boxed region-1 (b) and region-2 (c) in (a) [XIO 08].

1.7 The schematic diagram (a) of mechanical interlocking [GRU 03] and EDS image (b) of $\mathrm{Cu}$ (particle)/Al(substrate) [CHA 05] . . . . . . . . . . . . 8

1.8 Computed air and particle velocities as function of distance along the nozzle [STE 99]. . . . . . . . . . . . . . . . . . . . 15

1.9 Computed air and particle temperatures as function of distance along the nozzle [STE 99].

1.10 Different splat morphologies observed by SEM as a function of positions from the center of the particle jet: (a) near the center, (b) intermediate position and (c) near the rim.

1.11 Schematic diagram of the dependence of relative deposition efficiency on spray angle [LI 05]. . . . . . . . . . . . . . . . .

1.12 Etched Cross section micrographs of coatings obtained at a particle velocity of (a) $363 \mathrm{~m} / \mathrm{s}$ (b) $403 \mathrm{~m} / \mathrm{s}$ and (c) $469 \mathrm{~m} / \mathrm{s}$ [CHA 11]. . . . . . . . 18 
1.13 Porosity formation in cold sprayed microstructure for gas temperatures of (a) $300{ }^{\circ} \mathrm{C}$, (b) $420{ }^{\circ} \mathrm{C}$ and (c) $630{ }^{\circ} \mathrm{C}$ [ZAH 06]. . . . . . . . . . . 18

1.14 Effect of particle size distribution on porosity formation in cold spray process [ZAH 06].

1.15 Comparison of through-thickness residual stress distributions measured by the modified layer-removal technique for compacts produced from gas atomized and direct-reduction $\mathrm{Cu}$ powders [MCC 00] . . . . . . . . . . . 20

1.16 Measurement (symbols) and model fit (solid lines) of the through thickness in-plane stress distributions for (a) $\mathrm{Cu} / \mathrm{Cu}$ sample, (b) $\mathrm{Cu} / \mathrm{Al}$ sample, (c) $\mathrm{Al} / \mathrm{Cu}$ sample and (d) Al/Al sample [LUZ 11] . . . . . . . . . . . . 20

1.17 Residual stress in as-received specimens coated with Al7075 and pure Al [GHE 12]. . . . . . . . . . . . . . . . . 20

1.18 Temperature and curvature graphs of Ti/Carbon steel coating, illustrating the concepts of evolving, deposition, thermal and final residual stresses [SUH 13].

1.19 The erosive failure behavior of brittle and ductile materials: (a) erosion on brittle materials; (b) erosion on ductile materials [WAN 08] . . . . . . 21

1.20 Crater shape in (a) ductile and (b) brittle materials [RUF 79, AQU 01] . . . 21

1.21 Longitudinal section of the impact crater left in mild steel when hit by hardened steel ball $\mathrm{R}=4.75 \mathrm{~mm}, \alpha=30^{\circ}, v_{0}=141 \mathrm{~m} / \mathrm{s}$ : (a) material removal, (b) microphoto of special zone in the black rectangle indicated at (a) [HUT 76].

1.22 SEM observations for craters of Aluminum obtained by the impact tests of WC balls at impact angles of (a) $20^{\circ}$, (b) $30^{\circ}$, (c) $40^{\circ}$, (d) $60^{\circ}$ and (e) $90^{\circ}$ at an impact velocity of $100 \mathrm{~m} / \mathrm{s}$ [OKA 03 ] . . . . . . . . . .

1.23 A photograph of a crater caused by WC ball impact at an impact velocity of $2500 \mathrm{~m} / \mathrm{s}$ at $30^{\circ}$ for Aluminium [OKA 05c].

1.24 Cross-sectional surface of Aluminium at impact velocities of (a) $100 \mathrm{~m} / \mathrm{s}$, (b) $1320 \mathrm{~m} / \mathrm{s}$ and (c) $2270 \mathrm{~m} / \mathrm{s}$ [OKA 05c]. . . . . . . . . . . .

1.25 Scanning electron micrograph taken from periphery of erosion scar showing ductile erosion mechanisms for (a) 316 stainless steel eroded at 90 $\mathrm{m} / \mathrm{s}$ for a dwell time of $180 \mathrm{~s}, 30.4 \mu \mathrm{m}$ powder at $15^{\circ}$; (b) Stellite 12 eroded at $130 \mathrm{~m} / \mathrm{s}$ for a dwell time of $120 \mathrm{~s}, 30.4 \mu \mathrm{m}$ powder at $15^{\circ}$; (c) 316 stainless steel eroded at $130 \mathrm{~m} / \mathrm{s}$ for a dwell time of $180 \mathrm{~s}, 6.9 \mu \mathrm{m}$ powder at $15^{\circ}[\mathrm{AKB} 12] \ldots \ldots . \ldots \ldots 23$

2.1 SEM image of $\mathrm{Al}$ feedstock powder . . . . . . . . . . . . . 27

2.2 Schematic diagram of 2D axisymmetric model. . . . . . . . . . . 28

2.3 Schematic diagram of surface-to-surface contact. . . . . . . . . . . 33

2.4 Schematic diagram of boundary condition of 2D axisymmetry model. . . 33

2.5 Schematic diagram of initial conditions of velocity and temperature. . . . 34

2.6 Mesh structure of the 2D axisymmetry model. . . . . . . . . . . . . . 34 
2.7 Contours of (a) equivalent plastic strain and (b) temperature of Al/Al by using the Lagrangian numerical approach at $700 \mathrm{~m} / \mathrm{s}$ - aborted at $19 \mathrm{~ns}$. . 35

2.8 Element distortion of (a) Al particle and (b) Al substrate by using the Lagrangian numerical approach at $700 \mathrm{~m} / \mathrm{s} \ldots \ldots . \ldots 35$

2.9 Time histories of (a) equivalent plastic strain (PEEQ) and (b) temperature of Al/Al by using the Lagrangian numerical approach at $700 \mathrm{~m} / \mathrm{s}$. . . . 36

2.10 Schematic diagram of the ALE adaptive mesh domain. . . . . . . . . . 36

2.11 Schematic diagrams of detection and deactivation of geometric features. . 37

2.12 Contours of (a) equivalent plastic strain and (b) temperature of Al/Al by using the ALE numerical approach at $700 \mathrm{~m} / \mathrm{s} \ldots \ldots$. . . . . . . 38

2.13 Time histories of (a) equivalent plastic strain (PEEQ) and (b) temperature of Al/Al by using the ALE numerical approach at $700 \mathrm{~m} / \mathrm{s} \ldots \ldots 39$

2.14 Schematic diagram of 3D SPH model. . . . . . . . . . . . . . . 40

2.15 Schematic diagram of transformation of C3D8R element to PC3D elements. 42

2.16 Schematic diagram of kernel interpolate function. . . . . . . . . . . . . . 42

2.17 Schematic diagram of cube centred at the PC3D element. . . . . . . . . . 42

2.18 Contour of equivalent plastic strain of $\mathrm{Al} / \mathrm{Al}$ by using the SPH numerical approach at $700 \mathrm{~m} / \mathrm{s} \ldots \ldots \ldots \ldots . \ldots \ldots 4$

2.19 Time histories of (a) equivalent plastic strain (PEEQ) of $\mathrm{Al} / \mathrm{Al}$ and (b) stress of substrate by using the SPH approach at $700 \mathrm{~m} / \mathrm{s} \ldots \ldots$. . . . . . 44

2.20 Schematic diagram of 3D CEL model. . . . . . . . . . . . . . . 45

2.21 Boundary conditions for the 3D CEL model. . . . . . . . . . . . . . . 46

2.22 Contours of (a) volume average equivalent plastic strain of $\mathrm{Al}$ particle and (b) equivalent plastic strain of Al substrate by using the CEL numerical approach at $700 \mathrm{~m} / \mathrm{s} \ldots \ldots \ldots$. . . . . . . . . . . . . 48

2.23 Contours of (a) volume average temperature of $\mathrm{Al}$ particle and (b) temperature of Al substrate by using the CEL numerical approach at $700 \mathrm{~m} / \mathrm{s} . \quad 48$

2.24 Time histories of (a) equivalent plastic strain (PEEQ) and (b) temperature of Al/Al by using the CEL approach at $700 \mathrm{~m} / \mathrm{s} \ldots \ldots$. . . . . . . . . . 48

2.25 Time histories of normalized displacement of specific nodes by using the ALE approach. . . . . . . . . . . . . . . .

2.26 Time histories of (a) normalized kinetic energy and (b) normalized displacement of substrate center by using the four approaches at $700 \mathrm{~m} / \mathrm{s}$. . .

2.27 Time histories of (a) stable time increment and (b) artificial strain energy by using the four approaches at $700 \mathrm{~m} / \mathrm{s} \ldots \ldots \ldots$. . . . . . . 50

2.28 Evolution of Von Mises stress of $\mathrm{Al} / \mathrm{Al}$ at $500 \mathrm{~m} / \mathrm{s}$. (a) $5 \mathrm{~ns}$; (b) $10 \mathrm{~ns}$; (c) $20 n s$; (d) $30 n s ;$ (e) $40 n s$; (f) $60 n s . \ldots \ldots \ldots 1$

2.29 Evolution of mean stress of $\mathrm{Al} / \mathrm{Al}$ at $500 \mathrm{~m} / \mathrm{s}$. (a) $5 \mathrm{~ns}$; (b) $10 \mathrm{~ns}$; (c) 20

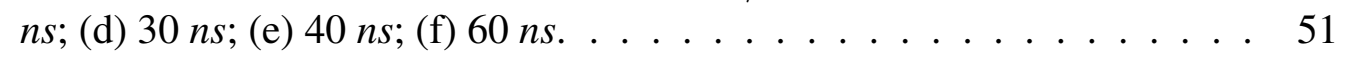

2.30 Evolution of equivalent plastic strain (PEEQ) of Al/Al at $500 \mathrm{~m} / \mathrm{s}$. (a) 5 $n s$; (b) $10 n s$; (c) $20 n s$; (d) $30 n s$; (e) $40 n s$; (f) $60 n s . \ldots 52$

2.31 Evolution of strain rate along the horizontal direction of contact surface of (a) Al particle and (b) Al substrate at $500 \mathrm{~m} / \mathrm{s} \ldots \ldots 52$ 
2.32 Evolution of (a) contact pressure (CPRESS) and (b) frictional shear stress (CSHEAR) of Al substrate impacted by an Al particle at $500 \mathrm{~m} / \mathrm{s}$. . . . 53

2.33 Evolution of temperature (TEMP) along the horizontal direction of contact surface of (a) Al particle and (b) Al substrate at $500 \mathrm{~m} / \mathrm{s}$. . . . . . . 53

2.34 Evolution of (a) contact force (CFORCE) and (b) normalized displacement of Al substrate impacted by an Al particle at $500 \mathrm{~m} / \mathrm{s}$. . . . . . . . 53

2.35 Deformation ratio and the position related to the maximum contact force of Al substrate impacted by an $\mathrm{Al}$ particle at $500 \mathrm{~m} / \mathrm{s} \ldots \ldots$. . . . . . 54

3.1 Schematic diagrams of path 1 and path $2 \ldots \ldots \ldots$. . . . . . . 57

$3.2 \mathrm{SEM}$ images of Al/Al at impact velocity of (a) $700 \mathrm{~m} / \mathrm{s}$, (b) $780 \mathrm{~m} / \mathrm{s}$ and

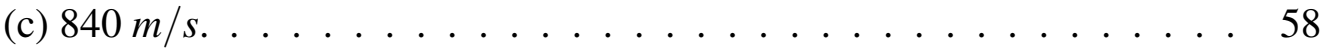

3.3 Simulation profiles of Al/Al at impact velocity of (a) $700 \mathrm{~m} / \mathrm{s}$, (b) 780 $m / s$ and (c) $840 \mathrm{~m} / \mathrm{s} \ldots \ldots \ldots \ldots$. . . . . . . . . . . . 59

3.4 Time histories of (a) equivalent plastic strain (PEEQ) and (b) temperature (TEMP) of Al/Al by using the CEL numerical approach at $700 \mathrm{~m} / \mathrm{s}, 780$ $m / s$ and $840 \mathrm{~m} / \mathrm{s} \ldots \ldots \ldots \ldots \ldots \ldots \ldots \ldots$

3.5 Developments of equivalent plastic strain (PEEQ) along the path 1 of $\mathrm{Al} / \mathrm{Al}$ by using the CEL numerical approach at (a) $700 \mathrm{~m} / \mathrm{s}$ and (b) 840 $m / s$.

3.6 Developments of temperature (TEMP) along the path 1 of Al/Al by using the CEL numerical approach at (a) $700 \mathrm{~m} / \mathrm{s}$ and (b) $840 \mathrm{~m} / \mathrm{s}$. . . . . . . .

3.7 Equivalent plastic strain (PEEQ) along the (a) path 1 and (b) path 2 of $\mathrm{Al} / \mathrm{Al}$ by using the CEL numerical approach at $700 \mathrm{~m} / \mathrm{s}, 780 \mathrm{~m} / \mathrm{s}$ and $840 \mathrm{~m} / \mathrm{s} \ldots \ldots \ldots \ldots \ldots \ldots \ldots \ldots$

3.8 Temperature (TEMP) along the (a) path 1 and (b) path 2 of Al/Al by using the CEL numerical approach at $700 \mathrm{~m} / \mathrm{s}, 780 \mathrm{~m} / \mathrm{s}$ and $840 \mathrm{~m} / \mathrm{s}$.

3.9 (a) SEM image and (b) simulation profile of $\mathrm{Cu} / \mathrm{Al}$ with an initial particle temperature of $873 \mathrm{~K}$.

3.10 Time histories of (a) equivalent plastic strain (PEEQ) and (b) temperature (TEMP) of $\mathrm{Cu} / \mathrm{Al}$ by using the CEL numerical approach at the initial particle temperatures of $473 K, 673 K$ and $873 K \ldots \ldots \ldots$ $\mathrm{Cu} / \mathrm{Al}$ by using the CEL numerocal approach at the initial particle temperatures of (a) $473 \mathrm{~K}$ and (b) $873 \mathrm{~K} \ldots \ldots \ldots 2$

3.12 Developments of temperature (TEMP) along the path 1 of $\mathrm{Cu} / \mathrm{Al}$ by using the CEL numerical approach at the initial particle temperatures of (a) 473 $K$ and (b) $873 K$.

3.13 Equivalent plastic strain (PEEQ) along the (a) path 1 and (b) path 2 of $\mathrm{Cu} / \mathrm{Al}$ by using the CEL numerical approach at the initial particle temperatures of $473 K, 673 K$ and $873 K$ 
3.14 Temperature (TEMP) along the (a) path 1 and (b) path 2 of $\mathrm{Cu} / \mathrm{Al}$ by using the CEL numerical approach at the initial particle temperatures of $473 \mathrm{~K}$, $673 K$ and $873 K$.

3.15 Simulation profiles of $\mathrm{Cu} / \mathrm{Cu}$ with friction coefficient of (a) 0.2 and (b) 0.5 by Li. et al [LI 09a] using the Lagrangian numerical approach. . . . . 63

3.16 Simulation profiles of $\mathrm{Cu} / \mathrm{Cu}$ with friction coefficients of (a) 0.2 and (b) 0.5 by using the CEL numerical approach. . . . . . . . . . . . . . . 64

3.17 Time histories of (a) equivalent plastic strain (PEEQ) and (b) temperature (TEMP) of $\mathrm{Cu} / \mathrm{Cu}$ by using the CEL numerical approach with friction coefficients of $0.2,0.3$ and $0.5 \ldots \ldots$. . . . . . . . . . . . 64

3.18 Time histories of friction energy (ALLFD) divided by internal energy (ALLIE) of $\mathrm{Cu} / \mathrm{Cu}$ by using the CEL numerical approach with friction coefficients of $0.2,0.3$ and $0.5 \ldots \ldots$. . . . . . . . . . . . 64

3.19 Developments of equivalent plastic strain (PEEQ) along the path 1 of $\mathrm{Cu} / \mathrm{Cu}$ by using the CEL numerical approach with friction coefficients

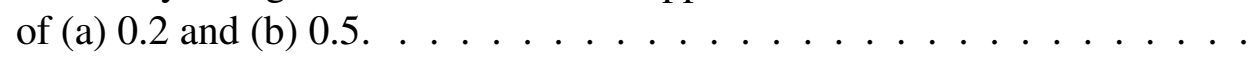

3.20 Developments of temperature (TEMP) along the path 1 of $\mathrm{Cu} / \mathrm{Cu}$ by using the CEL numerical approach with friction coefficients of (a) 0.2 and (b) 0.5. 65

3.21 Equivalent plastic strain (PEEQ) along the (a) path 1 and (b) path 2 of $\mathrm{Cu} / \mathrm{Cu}$ by using the CEL numerical approach with friction coefficients of

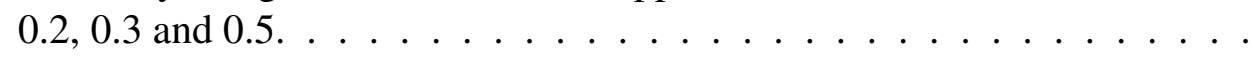

3.22 Temperature (TEMP) along the (a) path 1 and (b) path 2 of $\mathrm{Cu} / \mathrm{Cu}$ by using the CEL numerical approach with friction coefficients of $0.2,0.3$ and 0.5

3.23 (a) SEM image and (b) simulation profile of $\mathrm{Al} / \mathrm{Cu}$ at impact velocity of $500 \mathrm{~m} / \mathrm{s}$

3.24 Time histories of (a) equivalent plastic strain (PEEQ) and (b) temperature (TEMP) of $\mathrm{Al} / \mathrm{Al}, \mathrm{Cu} / \mathrm{Cu}, \mathrm{Al} / \mathrm{Cu}$ and $\mathrm{Cu} / \mathrm{Al}$ by using the $\mathrm{CEL}$ numerical approach at $500 \mathrm{~m} / \mathrm{s} \ldots \ldots \ldots \ldots \ldots$

3.25 Developments of (a) equivalent plastic strain (PEEQ) and (b) temperature (TEMP) along the path 1 of $\mathrm{Al} / \mathrm{Cu}$ by using the CEL numerical approach at $500 \mathrm{~m} / \mathrm{s}$.

3.26 Time histories of normalized kinetic energy by using the CEL numerical approach at $500 \mathrm{~m} / \mathrm{s}$. (a) $0-60 \mathrm{~ns}$; (b) $40-60 \mathrm{~ns}$.

3.27 Equivalent plastic strain (PEEQ) along the (a) path 1 and (b) path 2 of $\mathrm{Al} / \mathrm{Al}, \mathrm{Cu} / \mathrm{Cu}, \mathrm{Al} / \mathrm{Cu}$ and $\mathrm{Cu} / \mathrm{Al}$ by using the $\mathrm{CEL}$ numerical approach at $500 \mathrm{~m} / \mathrm{s} \ldots \ldots \ldots \ldots \ldots \ldots \ldots$

3.28 Temperature (TEMP) along the (a) path 1 and (b) path 2 of $\mathrm{Al} / \mathrm{Al}, \mathrm{Cu} / \mathrm{Cu}$, $\mathrm{Al} / \mathrm{Cu}$ and $\mathrm{Cu} / \mathrm{Al}$ by using the CEL numerical approach at $500 \mathrm{~m} / \mathrm{s}$. . . 68

3.29 Rebound velocities of (a) Al particle and (b) Al substrate at $500 \mathrm{~m} / \mathrm{s}$. . . 68 
3.30 Time histories of normalized kinetic energy of Al/Al by using the CEL numerical approach at $500 \mathrm{~m} / \mathrm{s}$. ALLKE - Kinetic energy; ALLIE - Total strain energy; ALLPD - Energy dissipated by rate-independent and ratedependent plastic deformation; ALLSE - Recoverable strain energy. . . .

3.31 Restitution coefficients of (a) different impact velocities, (b) different initial particle temperatures, (c)different friction coefficient and (d) different material combinations.

4.1 Schematic diagrams of three kinds of cubic unit cell, which are (a) simple cubic, (b) body center cubic and (c) face center cubic. . . . . . . . . . . .

4.2 Schematic diagram of the combination of two FCC particles arrangement models. . . . . . . . . . . . . . . . 73

4.3 Schematic diagram of the 3D CEL model with quarter symmetry. . . . . . 73

4.4 Landing location for a particle range between 5 and $60 \mu \mathrm{m}$ for a flat substrate, placed at a standoff distance of $10 \mathrm{~mm}$ [SAM 07] . . . . . . . . . 74

4.5 Secondary electron micrographs of the cross-section of (a) Al/Al and (b) $\mathrm{Cu} / \mathrm{Cu} \ldots \ldots \ldots \ldots \ldots 74$

4.6 Simulation profiles of (a) $\mathrm{Al} / \mathrm{Al}$ and (b) $\mathrm{Cu} / \mathrm{Cu}$ by using the $\mathrm{FCC}$ particles arrangement model. . . . . . . . . . . . . . . . 74

4.7 Simulation contours of pores for (a) $\mathrm{Al} / \mathrm{Al}$ and (b) $\mathrm{Cu} / \mathrm{Cu}$ by using the FCC particles arrangement model. . . . . . . . . . . . . . . 75

4.8 Schematic diagrams of (a) deposited particles and (b) cubic volume part for calculating porosity rate. . . . . . . . . . . 76

4.9 Simulation contours of pores for different process conditions of impact case $\mathrm{Cu} / \mathrm{Al}$. (a) case 4 - $600 \mathrm{~m} / \mathrm{s}, 473 \mathrm{~K}$; (b) case 5 - $650 \mathrm{~m} / \mathrm{s}, 473 \mathrm{~K}$; (c) case 6 - $600 \mathrm{~m} / \mathrm{s}, 523 \mathrm{~K}$; (d) case 7 - $650 \mathrm{~m} / \mathrm{s}, 523 \mathrm{~K} \ldots \ldots . . . . .76$

4.10 Simulation contours of pores for different process conditions of impact case $\mathrm{Cu} / \mathrm{Al}$. (a) case 4 - $600 \mathrm{~m} / \mathrm{s}, 473 \mathrm{~K}$; (b) case 5 - $650 \mathrm{~m} / \mathrm{s}, 473 \mathrm{~K}$; (c) case $6-600 \mathrm{~m} / \mathrm{s}, 523 \mathrm{~K}$; (d) case $7-650 \mathrm{~m} / \mathrm{s}, 523 \mathrm{~K} \ldots \ldots$. . . . . .

4.11 (a) Crack initiation along ASB in AISI 4340 steel subjected to high velocity impact [BAS 08], and (b) Void accumulation of ductile fracture in round notched bars of high strength steel [BEN 10]. . . . . . . . . . .

4.12 Simulation results of the through thickness mean stress distributions for (a) $\mathrm{Al} / \mathrm{Al}$, (b) $\mathrm{Al} / \mathrm{Cu}$, (c) $\mathrm{Cu} / \mathrm{Cu}$ and (d) $\mathrm{Cu} / \mathrm{Al} \ldots \ldots . . . . . . . .78$

4.13 Simulation results of the through thickness mean stress distributions for $\mathrm{Cu} / \mathrm{Al}$. (a) case $4-600 \mathrm{~m} / \mathrm{s}, 473 \mathrm{~K}$; (b) case 5 - $650 \mathrm{~m} / \mathrm{s}, 473 \mathrm{~K}$; (c) case $6-600 \mathrm{~m} / \mathrm{s}, 523 \mathrm{~K}$; (d) case $7-650 \mathrm{~m} / \mathrm{s}, 523 \mathrm{~K}$. . . . . . . . . . 79

5.1 Schematic diagram of contact angle. . . . . . . . . . . . . 83

5.2 Schematic diagrams of (a) one half model and (b) multiple oblique impacts model. . . . . . . . . . . . . . . . . 83

5.3 Schematic diagram of path $3 \ldots \ldots \ldots \ldots$. . . . . . . . . . 84

5.4 SEM images of $\mathrm{Cu} / \mathrm{Cu}$ with contact angle of (a) $50^{\circ}$ and (b) $70^{\circ} \ldots \ldots 5$ 
5.5 Simulation profiles of $\mathrm{Cu} / \mathrm{Cu}$ with contact angle of (a) $50^{\circ}$ and (b)70.$\quad$. 86

5.6 The simulation contours of equivalent plastic strain (PEEQ) of $\mathrm{Cu}$ substrate impacted by $\mathrm{Cu}$ particle with contact angle of $30^{\circ}$ (a,b), $50^{\circ}$ (c,d) and $70^{\circ}(\mathrm{e}, \mathrm{f})$.

5.7 The simulation contours of temperature (TEMP) of the $\mathrm{Cu}$ substrate impacted by a $\mathrm{Cu}$ particle with contact angle of $30^{\circ}(\mathrm{a}, \mathrm{b}), 50^{\circ}(\mathrm{c}, \mathrm{d})$ and $70^{\circ}$

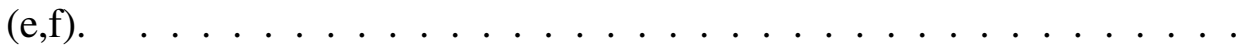

5.8 Time histories of the (a) equivalent plastic strain (PEEQ) and (b) temperature (TEMP) of the $\mathrm{Cu}$ substrate by using the CEL numerical approach with contact angle of $30^{\circ}, 50^{\circ}$ and $70^{\circ}$.

5.9 Development of the equivalent plastic strain (PEEQ) along the path 3 of the $\mathrm{Cu}$ substrate by using the CEL numerical approach with contact angle of (a) $30^{\circ}$ and (b) $70^{\circ}$.

5.10 Development of the temperature (TEMP) along the path 3 of the $\mathrm{Cu}$ substrate by using the CEL numerical approach with contact angle of (a) $30^{\circ}$ and (b) $70^{\circ}$.

5.11 Equivalent plastic strain (PEEQ) and temperature (TEMP) along the path 3 of the $\mathrm{Cu}$ substrate by using the CEL numerical approach with contact angle of $30^{\circ}, 50^{\circ}$ and $70^{\circ}$.

5.12 Simulation profiles of surface erosion of $\mathrm{Cu}$ substrate impacted by $\mathrm{Cu}$ particle with contact angle of (a) $30^{\circ}$, (b) $50^{\circ}$ and (c) $70^{\circ}$.

5.13 The evolution of contact surface of $\mathrm{Cu}$ substrate impacted by a $\mathrm{Cu}$ particle with contact angle of $30^{\circ}$. (a) $36 \mathrm{~ns}$; (b) $42 \mathrm{~ns}$; (c) $48 \mathrm{~ns}$; (c) $60 \mathrm{~ns} . \quad \ldots .89$

5.14 Simulation results of erosion rate and indentation versus contact angle. . . 89

5.15 Impact of the $\mathrm{Cu}$ substrate by a $\mathrm{Cu}$ particle at $500 \mathrm{~m} / \mathrm{s}$ with various contact angles. (a) Time history of normalized kinetic energy; (b) Time history of normalized temperature rise. . . . . . . . . . . . . . 90

5.16 Simulation results of erosion rate and indentation versus impact velocity. . 90

5.17 Impact of the $\mathrm{Cu}$ substrate by a $\mathrm{Cu}$ particle at various impact velocities with contact angle of $50^{\circ}$. (a) Time history of normalized kinetic energy;

(b) Time history of normalized temperature rise. . . . . . . . . . . . . 90

5.18 Simulation results of erosion rate and indentation versus friction coefficient. 91

5.19 Impact of the $\mathrm{Cu}$ substrate by a $\mathrm{Cu}$ particle at $500 \mathrm{~m} / \mathrm{s}$ with various friction coefficient. (a) Time history of normalized kinetic energy; (b) Time history of normalized temperature rise. . . . . . . . . . . .

5.20 Impact of the $\mathrm{Cu}$ substrate by multiple $\mathrm{Cu}$ particle at $500 \mathrm{~m} / \mathrm{s}$ with contact
angle of $50^{\circ}$. (a) Deformation shape of particles; (b)Deformation shape of substrate.

5.21 The evolution of contact domain of $\mathrm{Cu}$ substrate impacted by multiple $\mathrm{Cu}$ particle at $500 \mathrm{~m} / \mathrm{s}$. (a) $12 \mathrm{~ns}$; (b) $32 \mathrm{~ns}$; (c) $76 \mathrm{~ns}$; (d) $100 \mathrm{~ns} . . . .$. . . .

5.22 The schematic diagrams of (a) tensile failure mode and (b) shear failure mode. 
5.23 The crack propagation within $\mathrm{Cu}$ substrate impacted by multiple $\mathrm{Cu}$ particles at $500 \mathrm{~m} / \mathrm{s}$. (a) $12 \mathrm{~ns}$; (b) $32 \mathrm{~ns}$; (c) $76 \mathrm{~ns}$; (d) $100 \mathrm{~ns}$. . . . . . . . . 92

AA.1 Time histories of (a) displacement at the particle center, (b) velocity at the particle center, (c) temperature at the particle center and (d) kinetic energy of particle. . . . . . . . . . . . . . . . . 100

AC.1 Simulated contours of PEEQ (a) and TEMP (b) of Al/Al using the DynamicExplicit including adiabatic heating procedure. . . . . . . . . . . 106

AC.2 Simulated contours of PEEQ (a) and TEMP (b) of Al/Al using the Dynamic Temp-Disp Explicit procedure. . . . . . . . . . . . . . . 106

AC.3 Time histories of (a) PEEQ and (b) TEMP of Al/Al by using two different analysis procedures at $500 \mathrm{~m} / \mathrm{s} \ldots \ldots \ldots$. . . . . . . . . . . . . . 106

AD.1 Schematic diagram of basic Coulomb friction model. . . . . . . . . . . 107

AD.2 Schematic diagram of master surface penetrations into the slave surface. . 109

AD.3 Schematic diagram of finite sliding formulation. . . . . . . . . . . . . 109

AD.4 Schematic diagrams of (a) global search and (b) local search. . . . . . . 110

AE.1 Schematic diagram of relocation of a node during a mesh sweep. . . . . . 111

AE.2 Schematic diagram of enforcing a spatial mesh constraint. . . . . . . . . 112

AE.3 Schematic diagram of second-order advection. . . . . . . . . . . . . . 112

AG.1 Procedure for using the volume fraction tool. . . . . . . . . . . . . 121

AH.1 Topology of an eroding contact surface. . . . . . . . . . . . . . . . 123

AI.1 A typical uniaxial stress-strain response of a ductile metal specimen. . . . 126

AI.1 Impact of $\mathrm{Al}$ substrate by multiple $\mathrm{Cu}$ particles at $500 \mathrm{~m} / \mathrm{s}$ with contact angle of $50^{\circ}$. (a) Deformation profile of particles by using the progressive damage and failure model; (b) Deformation profile of substrate by using the progressive damage and failure model. . . . . . . . . . . . 128

AI.2 Impact of Al substrate by multiple $\mathrm{Cu}$ particles at $500 \mathrm{~m} / \mathrm{s}$ with contact angle of $50^{\circ}$. (a) Deformation profile of particles by using the dynamic failure model; (b) Deformation profile of substrate by using the dynamic failure model. . . . . . . . . . . . . . . . . . . . 129 


\section{List of Tables}

1.1 Influence of temperature on the deposition of $\mathrm{Al}$ particles impinging with high velocities onto substrates of $\mathrm{Cu}$ and steel [KLI 05] . . . . . . . . . 16

2.1 Material properties of Aluminum used in the CEL analysis. . . . . . . . 30

2.2 The influences of frequency and intensity on the simulation results at 700

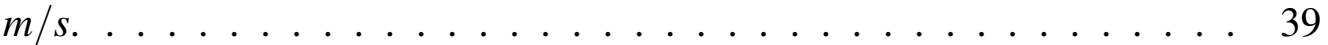

2.3 Comparison of computational cost by using four approaches. . . . . . . 50

3.1 Calculation plan for investigating the effects of parameters. . . . . . . . . 57

3.2 Material properties of Aluminum and Copper used in the CEL simulation. 58

3.3 Schematic illustration of the effects of parameters including Velocity, Temperature and Friction coefficient. . . . . . . . . . . . . . . . . 69

4.1 Calculation plan for investigating multiple impacts. . . . . . . . . . . 72

4.2 Estimated impact parameters based on the simulations of CEL numerical approach. ......................... 75

4.3 comparison of predicted volume porosity rate and experiment results. . . 77

5.1 Calculation plan for the simulation of oblique impact. . . . . . . . . . 83

5.2 Material properties of copper used in the simulation. . . . . . . . . . 85

C.1 Schematic illustration of effects of parameters. . . . . . . . . . . . 96

C.2 Material properties of aluminum and copper used in the simulation. . . . 128 


\section{List of Tables}

xiv

Cette thèse est accessible à l'adresse : http://theses.insa-lyon.fr/publication/2014ISAL0044/these.pdf (C) [J. Xie], [2014], INSA de Lyon, tous droits réservés 


\section{Introduction}

This cold spray project is based on a very close collaboration between the LaMCoS laboratory of INSA de Lyon and the Fracture and Reliability Research Institute of TOHOKU university of Japan under the framework of the Elyt Laboratory. This project focuses on the cold spray coating process and its numerical simulation, combining experimental and modeling competences. The primary work of French side is numerical simulation of cold spray particle deposition process, which is the work I performed in this thesis.

Cold spray as a coating technology was initially developed in the mid-1980s at the Institute for Theoretical and Applied Mechanics of the Siberian Division of the Russian Academy of Science in Novosibirsk. The Russian scientists successfully deposited a wide range of pure metals, metallic alloys, polymers, and composites onto a variety of substrate materials, and they demonstrated that very high coating deposition rates are attainable using the cold spray process. Today, a variety of cold spray research is being conducted at institutions in dozens of locations world wide, and cold spray is increasingly being used in a number of industries, including aerospace, energy, and military.

Traditional thermal spray coating forming process involves heating the particle material to very high temperatures, often higher than the melting temperature. Owing to the high operating temperatures, the presence of porosity, oxides and residual tensile stress in the thermal spray coating are inevitable. These imperfections can significantly degrade the mechanical, electrical and thermal properties of coating. Thus limitations of the traditional thermal spray process have been the cause of thermally induced failure in engineering components.

The distinguishing features of cold spray include the ability to form dense deposits with extremely low oxygen content, free of residual tensile stresses, grain growth, recrystallization zones, and phase changes. Certain materials may even experience grain refinement at the nanometer scale. Because adhesion of the metal powder to the substrate and deposited material is achieved in the solid state, the characteristics of cold spray deposits are quite unique, making cold spray suitable for depositing a wide range of traditional and advanced materials on many types of substrate materials, especially in non-traditional applications that are sensitive to the temperature of the process. The cold spray coating technology and preceding researches on it are described in detail in Chapter 1.

The impact velocity of cold spray particles ranges between $300 \mathrm{~m} / \mathrm{s}$ and $1200 \mathrm{~m} / \mathrm{s}$, and the common contact time is about tens of nanoseconds. How to simulate the material deformation behaviors with high strain rate (up to $10^{8}$ to $10^{9} s^{-1}$ ), therefore, is the 
first scientific challenge we met in this study. In Chapter 2, details of the four numerical approaches - Lagrangian, Arbitrary Lagrangian Eulerian (ALE), Smoothed Particle Hydrodynamics (SPH), Coupled Eulerian Lagrangian (CEL) - used for solving this problem are outlined. The feasibility of each numerical approach is verified through a simple case, which is an Aluminum particle of $25 \mu \mathrm{m}$ impacting on the Aluminum substrate at impact velocity of $700 \mathrm{~m} / \mathrm{s}$.

The bonding of particles in cold spray is presumed to be the result of extensive plastic deformation and related phenomena at the interface. Despite the adiabatic shear instability is generally accepted to be responsible for successful bonding, it has not yet become clear what are the essential bonding mechanisms between particle and substrate. The key parameters for successful bonding are impact velocity and temperature. High velocity is necessary for optimal deposition efficiency and packing density. At low temperature, the particle oxidation is avoided, thus the cold spray coatings are more durable with better bond strength. Besides, the velocity and temperature are also important parameters which affect the porosity rate of coating and residual stress. What is the influence of the friction coefficient on the mutual contact between particle and substrate? The materials of coating and substrate could be divided into four groups based on the concept of hardness, which are hard/hard, soft/soft, hard/soft and soft/hard. Different material combinations have different deformation behaviors. Chapters 3 through 4 consider the effects of these parameters in detail with the single impact model and multiple impacts model using the Coupled Eulerian Lagrangian method.

Simulation of successful deposition process is necessary, simulation of surface erosion induced by extremely high velocity or low contact angle is also considered in this study. The Shear failure and Tensile failure models are first introduced in Chapter 5 in order to simulate the surface erosion of substrate materials. In this Chapter, the influence of contact angle, velocity and friction coefficient on the volume wear rate are demonstrated, and a preliminary investigation on the fracture propagation of the substrate is given. 


\title{
Chapter 1
}

\section{Previous researches on the cold spray deposition process}

\author{
A brief description of cold spray coating technology is \\ presented, as well as the four numerical methods used for \\ modeling high speed impact problem. This chapter intends to \\ review some previous simulation works mostly concerning the \\ influences of cold spray parameters, which are velocity, \\ temperature, and contact angle, on the deposition behavior of \\ particle materials. This chapter also includes a description of \\ experimental results of cold spray focusing on the porosity \\ rate, residual stress and erosion damage.
}

\section{Contents}

1.1 Cold spray coating technology $\ldots \ldots \ldots \ldots \ldots \ldots$

1.1.1 Coating formation process $\ldots \ldots \ldots \ldots$

1.1.2 Advantages and disadvantages . . . . . . . . . . . . . 5

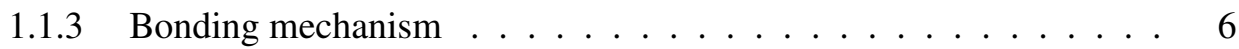

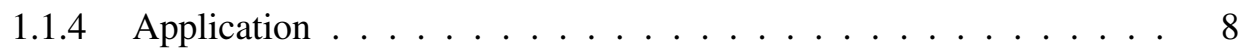

1.2 Numerical approach $\ldots \ldots \ldots \ldots \ldots \ldots$

1.2 .1 Lagrangian . . . . . . . . . . . . . . . . 9

1.2.2 Arbitrary Lagrangian Eulerian _... . . . . . . . . . . . 9 
1.2.3 Smoothed Particle Hydrodynamics . . . . . . . . . . . . . . . . 10

1.2.4 Coupled Eulerian Lagrangian _. . . . . . . . . . . . . . 10

1.3 Previous studies $\ldots \ldots \ldots \ldots \ldots \ldots \ldots$

1.3.1 Simulation of cold spray $\ldots \ldots \ldots \ldots \ldots \ldots$

1.3.2 Effect of parameters . . . . . . . . . . . . . . 14

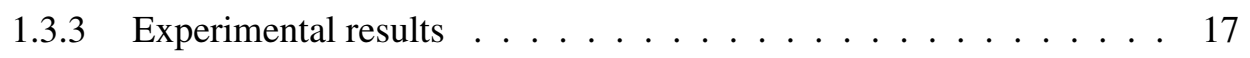

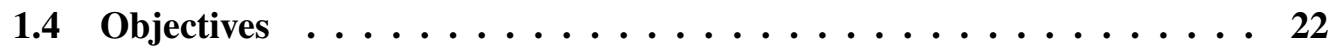




\subsection{Cold spray coating technology}

\subsubsection{Coating formation process}

Cold spray is a process whereby metal powder particles are utilized to form a dense coating by means of ballistic impingement upon a suitable substrate. The metal powders range in particle size from 5 to $100 \mu \mathrm{m}$ and are accelerated by injection into a high velocity stream of gas. The high velocity gas stream is generated through the expansion of a pressurized, preheated gas through a converging-diverging nozzle. The pressurized gas is expanded to supersonic velocity, with an accompanying decrease in pressure and temperature. The powder particles, initially carried by a separate gas stream, are injected into the nozzle either prior to the throat or downstream of the throat. The particles are then accelerated by the main nozzle gas flow and are impacted onto a substrate after exiting the nozzle. Upon impact, the solid particles deform and create a bond with the substrate. As the process continues, particles continue to impact the substrate and form bonds with the deposited material, resulting in an uniform coating with very little porosity and high bond strength. FIG.1.1 shows a schematic diagram of cold spray apparatus, and FIG.1.2 gives a clearer view on the stages of coating formation in the cold spray deposition process [STE 02]. The term 'cold spray' has been used to describe this process due to the relatively low temperatures $\left(-100\right.$ to $\left.+100{ }^{\circ} \mathrm{C}\right)$ of the expanded gas stream that exits the nozzle.

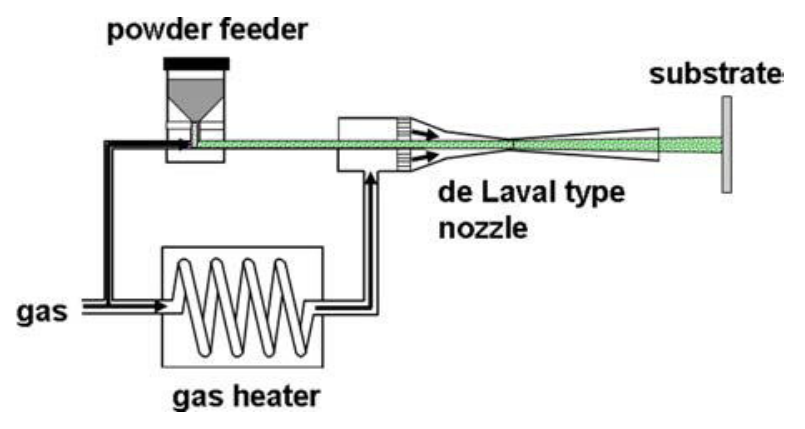

Figure 1.1: The schematic diagram of cold spray apparatus.

\subsubsection{Advantages and disadvantages}

As with any other materials processing technique, the cold spray process has its own advantages and disadvantages. The main advantage of the cold spray process is that it is a solid-state process, which results in many unique coating characteristics. The main disadvantage arises due to the plastic deformation process, which leads to a loss of ductility of the coating. Some advantages and disadvantages are displayed below [CHA 07].

Advantages:

- High deposition efficiency, high deposition rate, high density, high bond strength, high conductivity, high corrosion resistance, high strength and hardness; 


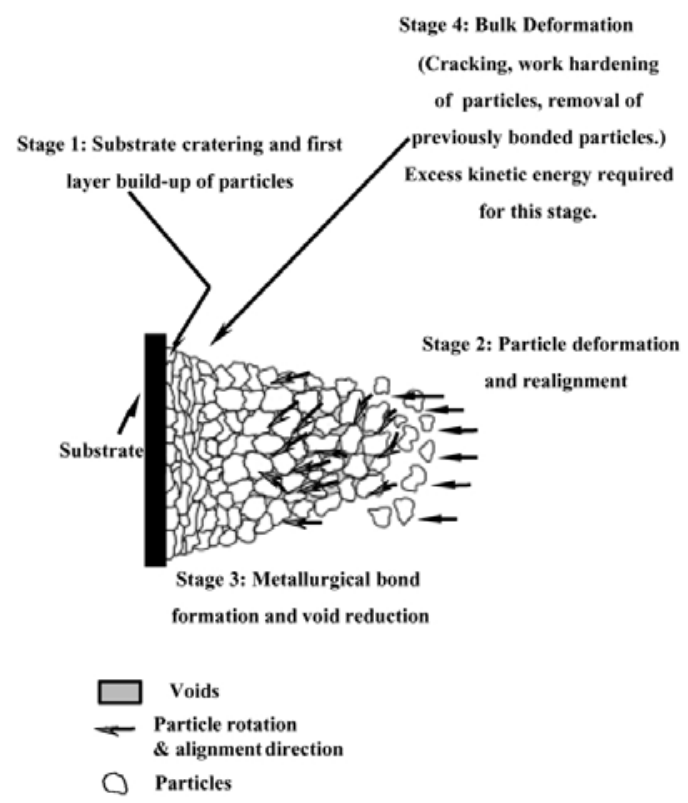

Figure 1.2: The stages of coating formation in the cold spray deposition process [STE 02].

- No or little masking, no grit blast, no phase changes, no oxidation, no grain growth;

- Flexibility in substrate-coating selection;

- Minimum thermal input to the substrate;

- Compressive residual stresses;

- Ultra-thick coatings.

\section{Disadvantages:}

- Pure ceramics and some alloys (such as work-hardening alloys) can not be processed;

- Cold-sprayed coatings over ceramic substrates show only limited bond strength;

- High quality coatings are produced with expensive helium as the processing gas in order to achieve the velocities necessary for deposition;

- Spraying complex shapes and internal surfaces is difficult.

\subsubsection{Bonding mechanism}

The bonding mechanisms in cold gas spraying can be compared to those in processes such as explosive cladding or shock wave powder compaction due to the bonding is the result of extensive plastic deformation and related phenomena at the interface. In explosive cladding, successful bonding occurs within a certain range of impact angles, impact velocities and materials properties, and it is often manifested by the formation of an out-flowing jet of material at the contact zone [HEN 13]. Within a distance of a few millimetres from the interface, there is a sequence of regions with severe deformation, highly elongated grains, recrystallised grains, and sometimes resolidified microstructures, though melting 
and resolidification are often limited to a thickness of less than a micrometer at the interface (FIG.1.3). In explosive powder compaction, dense solids of materials ranging from metals such as Aluminum, steels and nickel based superalloys, to ceramics can be produced by appropriate selection of shock pressure and duration [BUZ 13]. In analogy with explosive cladding, successful bonding in powder compaction, also, has been related to critical conditions for extensive plastic deformation at the particle/particle interface (FIG.1.4).
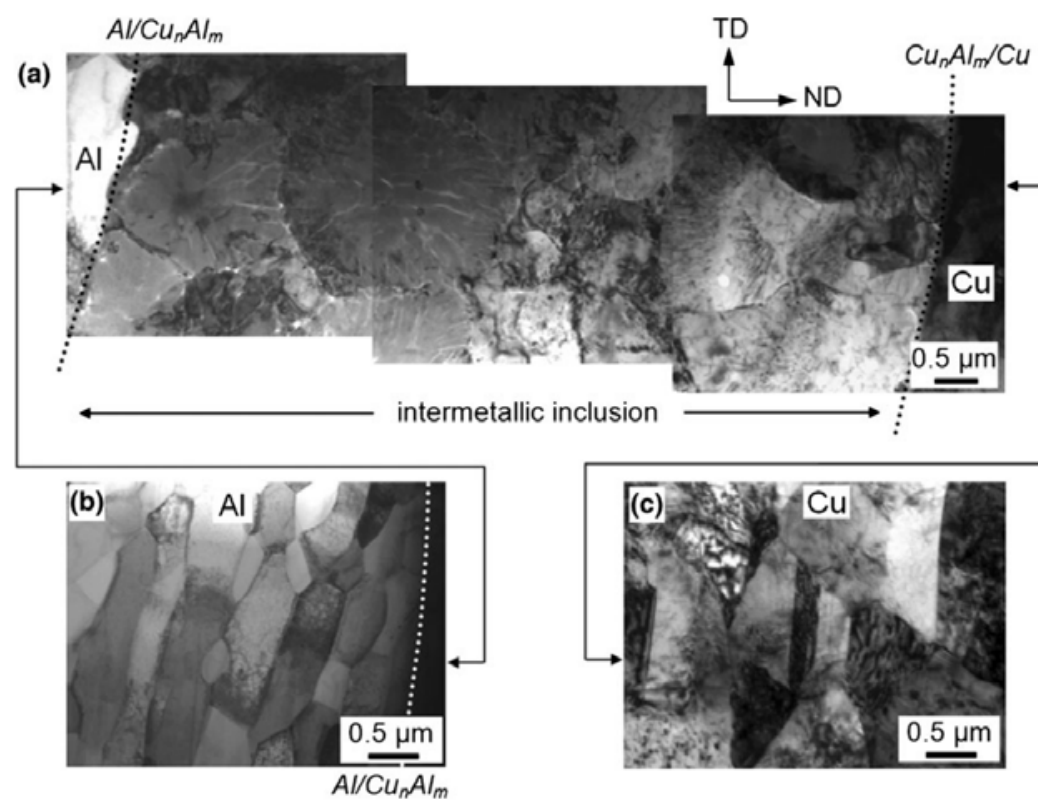

Figure 1.3: Microstructural changes across the interfacial zone. (a) Dendrites inside the intermetallic inclusion. Microstructures of the base plates in areas directly adhering to the intermetallic inclusion: (b) elongated subgrains inside the aluminum sheet and (c) ?ne grains inside copper sheet. TEM bright ?eld images in the ND-TD section [HEN 13].

(a)

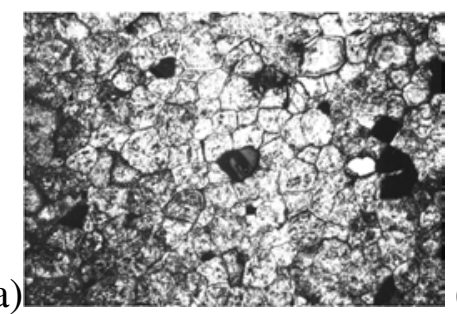

(b)

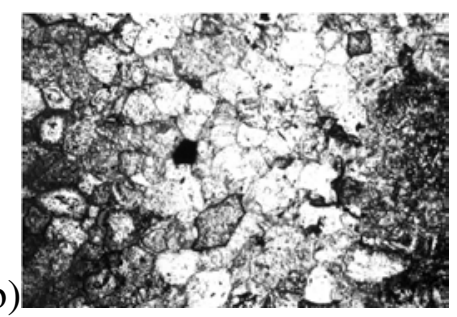

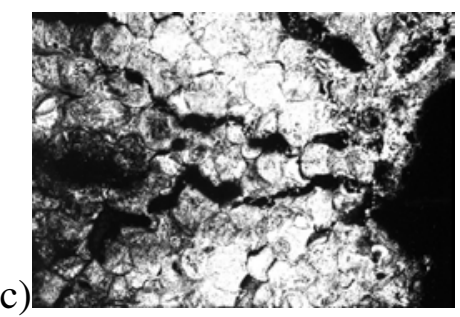

Figure 1.4: The structures of the cross sections of the samples for three different values of Detonation velocity (D) and at the same thickness of explosive charge ? $5 \mathrm{~mm}$ : (a) $\mathrm{D}=3.19 \mathrm{~km} / \mathrm{s}$; (b) $\mathrm{D}=3.95 \mathrm{~km} / \mathrm{s}$; (c) $\mathrm{D}=5.26 \mathrm{~km} / \mathrm{s}$ [BUZ 13].

A number of phenomena - such as interfacial melting, atomic inter-diffusion, plastic deformation [KLI 05], etc. - are now believed not to play a significant role in the particle/substrate bonding during cold spray deposition process. The average kinetic energy 
of depositing particles at impact is typically significantly lower than the energy required to melt the particle/substrate interfacial region, suggesting that particle/substrate bonding is primarily a solid-state process. The lack of melting was directly confirmed through micrographic examination of the cold sprayed materials [GRU 03, OGA 08, LEE 04]. The metal-metal inter-diffusion coefficient at temperatures near the melting point is of the order of $10^{-15}$ to $10^{-13} \mathrm{~m}^{2} / \mathrm{s}$, and for a typical particle/substrate contact time of $40 \mathrm{~ns}$, the atomic inter- diffusion distance is between 0.004 and $0.1 \mathrm{~nm}$. Since this distance is only a fraction of the inter-atomic distance, atomic diffusion at the particle/substrate interface should be excluded as a dominant particle/substrate bonding mechanism. Plastic deformation is generally considered to be a source of heat in the interfacial region due to the energy dissipation. However, as mentioned above, the temperature increase in the interfacial region is not sufficient to produce interfacial melting or to significantly promote atomic inter-diffusion, thus leaving this point plays a relatively minor role in particle/substrate interfacial bonding.

Adiabatic shear instability and the resultant plastic flow localization, however, are the phenomena that are believed to play a major role in the particle/substrate bonding during the cold spray deposition process [ASS 03, GRU 04]. Adhesion requires clean surfaces and relatively high contact pressures to make the surfaces mutually conforming. Adiabatic shear localization and the associated formation of the interfacial jets during cold spraying can be expected to produce clean contacting surfaces. In addition, adiabatic softening of the material in the particle/substrate interfacial region combined with relatively high contact pressures promote formation of mutually conforming contacting surfaces via plastic deformation of the contacting surfaces. Hence, once the conditions for the onset of adiabatic shear localization (and adiabatic softening) are attained at sufficiently high impact particle velocities, the conditions for extensive adhesion of the particle and substrate surfaces are reached resulting in particle/substrate bonding. Typical dynamic stress-strain curves are shown in FIG.1.5(a). For a typical work-hardening material under non-adiabatic conditions, the stress-strain curve (denoted as 'Isothermal' in FIG.1.5(a)) shows a monotonic increase of the flow stress with plastic strain. However, under adiabatic conditions, the plastic strain energy dissipated as heat increases the temperature causing material softening. The rate of strain hardening decreases and the flow stress reaches a maximum value, past which a monotonic decreases in the flow stress with plastic strain takes place (the curve labelled 'Adiabatic' in FIG.1.5(a)). The fluctuations in stress, strain, temperature or microstructure, and the inherent instability of strain softening can give rise to plastic flow (shear) localization. Consequently, the straining and heating in the surrounding material regions practically stop. This, in turn, causes the flow stress to quickly drop to zero (the curve denoted 'Localization' in FIG.1.5(a)). Simple schematics are used to explain this mechanism in FIG.1.5(b). XIONG [XIO 08] reported dynamic amorphization and recrystallization processes of metals upon impact of micron-scaled particles at a high strain rate $10^{9} s^{-1}$ combining adiabatic heating with rapid cooling in a kinetic spray process. At the interface of the particle/substrate, an amorphous zone with a thickness of about $3 \mathrm{~nm}$ was observed (FIG.1.6) after individual particle impact. It is consistent with the mechanism of adiabatic shear instability characteristics in kinetic spray 
process.

CHAMPAGNE's experimental observation of high interfacial bonding strengths [CHA 05] suggests that, in addition to adhesion mechanism discussed earlier, some type of nano/micro length-scale mechanical material mixing/interlocking mechanism may also contribute to particle/substrate bonding, as shown in FIG.1.7(b). Such interfacial roll-ups and vortices may enhance the overall strength of interfacial bonding by at least three ways: (a) by significantly increasing the interfacial area available for adhesion; (b) by producing a fine length-scale mixing of the two materials; and (c) by creating mechanical interlocking between the two materials [GRU 03].

(a)

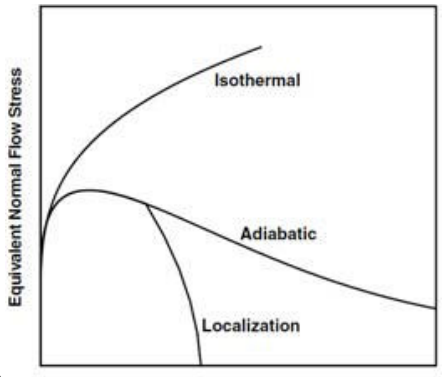

Equivalent Normal Plastic Strain

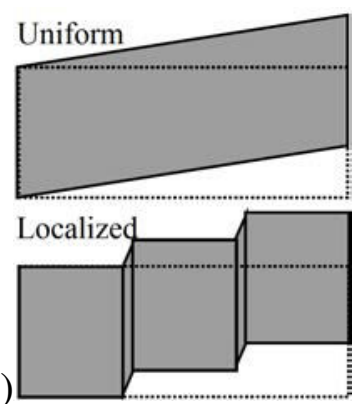

(b)

Figure 1.5: (a) Typical stress-strain curves in a normal strainhardening material ('Isothermal'), an adiabatically softened material ('Adiabatic') and in a material undergoing an adiabatic shear localization ('Localization'); (b) schematics of the uniform and the localized simple shears [CHA 05].

(a)

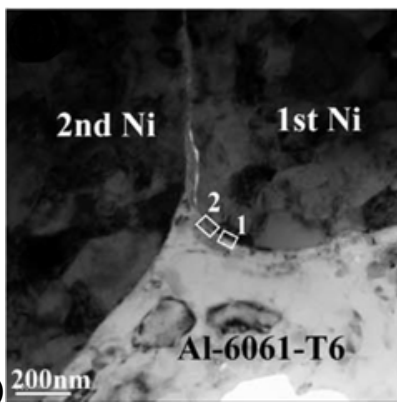

(b)

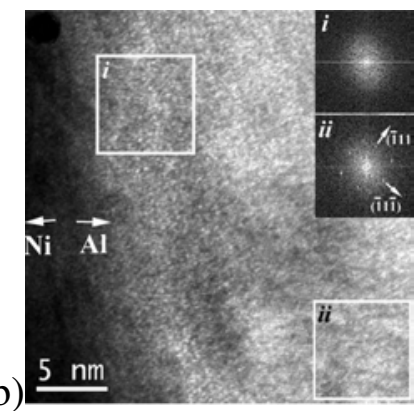

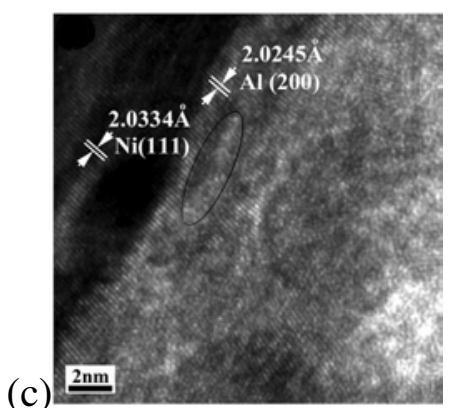

Figure 1.6: (a) Low-magnification TEM image of interfacial microstructures between as-sprayed Ni coatings and Al-6061-T6 alloy substrate in a kinetic spray process. HREM images of boxed region-1 (b) and region-2 (c) in (a) [XIO 08].

\subsubsection{Application}

There are many applications for cold spray coating, some of them are listed as below [MAR 06]:

- Corrosion Resistant Coatings (Zn, Al); 


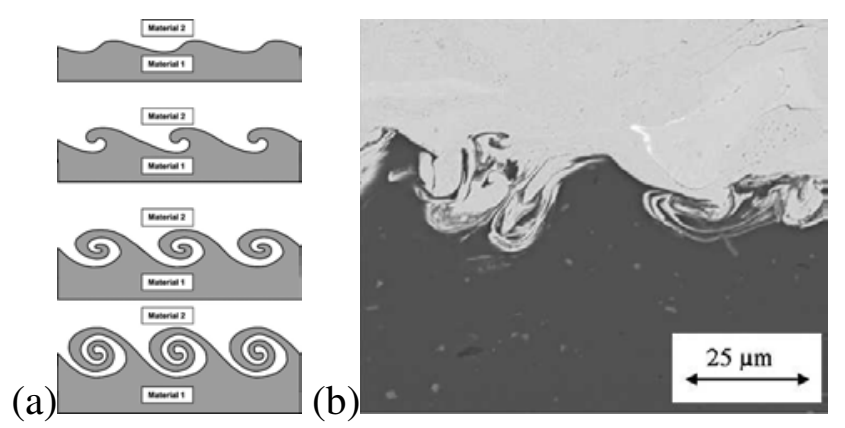

Figure 1.7: The schematic diagram (a) of mechanical interlocking [GRU 03] and EDS image (b) of $\mathrm{Cu}$ (particle)/Al(substrate) [CHA 05].

- Dimensional Restoration and Repair (Ni, Stainless Steel, Ti, Al);

- Wear Resistant Coatings (CrC-NiCr, WC-Co, WCu);

- Portable Units for Field Repair;

- Biomedical: prostheses with improved wear resistance;

- Aerospace: fatigue-resistant coatings;

- Chemical: improved corrosion resistance;

- Mineral processing: improved corrosion and erosion resistance;

- Die casting: extending die life;

- Electronics: creating a heat sink or superconductive, magnestostrictive surfaces;

- Printing: $\mathrm{Cu}$ coating on rollers;

- Oil and gas: improved corrosion resistance;

- Glass: platinum coating.

\subsection{Numerical approach}

Due to their highly transient non-linear dynamic and contact nature, the interactions between particles and the substrate during the cold spray deposition process are very difficult to analyze using experimental ways. Micro-structural and micro-analytical examinations have difficulties in helping to identify the relative contribution of various bonding mechanisms. As a result, computational simulations of the particle/substrate interactions have been an important technique for elucidating the bonding mechanism accompanying cold spray. Consequently, a brief overview is given concerning the fundamental concepts of numerical approach used in this study.

\subsubsection{Lagrangian}

The movement of the continuum is specified as a function of the material coordinates and time, i.e. the nodes of the Lagrangian mesh move together with the material. Conservation of mass is automatically satisfied and material boundaries are clearly defined, and the interface between two parts is precisely tracked and defined. This is ideal for 
following the material motion and deformation in regions of relatively low distortion, and possibly large displacements. The Lagrangian method is more appropriate for representing the solids like structures. The advantages of the Lagrangian numerical approach are computation efficiency and ease of incorporating complex material models. The shortcoming of this approach is that the extremely severe distortions in the deformed region, which could be lead to adverse effects on the accuracy and convergence. These problems can be overcome to a certain extent by applying numerical technique such as rezoning [GRU 04, LI 09b, LI 09a, YIL 11, SHU 09, YIN 10, QIU 11].

\subsubsection{Arbitrary Lagrangian Eulerian}

The Arbitrary Lagrangian Eulerian (ALE) numerical approach combines the features of pure Lagrangian analysis and pure Eulerian analysis. ALE adaptive meshing is a tool that makes it possible to maintain a high quality mesh throughout an analysis, even when large deformation or loss of material occurs, by allowing the mesh to move independently of the material. ALE adaptive meshing does not alter the topology (elements and connectivity) of the mesh. It allows redefining the grid continuously in arbitrary and predefined ways as the calculation proceeds, which effectively provides a continuous rezoning facility. Adaptive meshing is particularly effective for simulations of metal forming processes such as forging, extrusion, and rolling because these types of problems usually involve large amounts of nonrecoverable deformation. Because the final shape of the product can be drastically different from the original shape, a mesh that is optimal for the original product geometry can become unsuitable in later stages of the process when large material deformation leads to severe element distortion and entanglement. Element aspect ratios can also degrade in zones with high strain concentrations. Both of these factors can lead to a loss of accuracy, a reduction in the size of the stable time increment, or even termination of the problem. Compared with Lagrangian, an additional computational step of rezoning is employed, thus the computation cost rises. Moreover, the simualtion results are largely dependent on the choice of parameters, such as remeshing region, frequency, remeshing sweeps per increment, etc.[LI 09b, LI 09a, YIL 11, ASS 03, BAE 08, LI 07b].

\subsubsection{Smoothed Particle Hydrodynamics}

The Smooth Particles Hydrodynamics (SPH) is a meshfree, adaptive, Lagrangian computational approach for problem solving in Computational Continuum Dynamics extended to treat the dynamic response of solids. In the SPH reference frame, the problem domain is represented by a set of non-connected particles which possess unique material properties. The SPH particles are not only interacting mass points but also interpolation points used to calculate the value of physical parameters based on the data from neighboring SPH particles, scaled by a weighting function. Because there is no mesh defined, the SPH numerical approach does not suffer from mesh distortion or tangling in large deformation problems. Material boundaries and interfaces in the SPH are well defined and material separation is naturally handled. The Lagrangian nature, associated with the 
absence of a fixed mesh, is its main strength. Difficulties associated with fluid flow and structural problems involving large deformations and free surfaces are solved in a relatively natural way. Fluid sloshing, wave engineering, ballistics, spraying (as in paint spraying), gas flow, and fragmentation followed by secondary impacts are a few examples of application of SPH numerical approach. Because the SPH numerical approach requires a sort of particles in order to locate current neighboring particles, this makes the computation time per cycle more expensive than mesh based Lagrangian techniques [YIN 10, LI 10b, MAN 12, MAN 11a, GUI 11].

\subsubsection{Coupled Eulerian Lagrangian}

In a traditional Lagrangian analysis the nodes are fixed within the material, and elements deform as the material deforms. Lagrangian elements are always $100 \%$ full of a single material, so the material boundary coincides with an element boundary. By contrast, in an Eulerian analysis the nodes are fixed in space, and material flows through elements that do not deform. Eulerian elements may not always be $100 \%$ full of material, many may be partially or completely void. The Eulerian material boundary must, therefore, be computed during each time increment and generally does not correspond to an element boundary. Eulerian material can interact with Lagrangian elements through Eulerian-Lagrangian contact; simulations that include this type of contact are often referred to as coupled Eulerian Lagrangian (CEL) analysis [SMO 11, QIU 11]. In this analysis, material is tracked as it flows through the mesh by computing its Eulerian Volume Fraction (EVF) within each element. By definition, if a material completely fills an element, its volume fraction is one; if no material is present in an element, its volume fraction is zero. Eulerian elements may simultaneously contain more than one material. If the sum of all material volume fractions in an element is less than one, the remainder of the element is automatically filled with void. Void material has neither mass nor strength [DAS11]. The CEL numerical approach allows complex fluid-structure interaction problems including large displacements and deformations of the structure, to be solved in a single numerical simulation. It is hard to understand the history of material deformation due to the nature of Eulerian analysis, as well as to identify the interface between two parts (Eulerian-Eulerian contact or Eulerian-Lagrangian contact).

\subsection{Previous studies}

\subsubsection{Simulation of cold spray}

ASSADI [ASS 03] of Tarbiat Modarres University (Iran) used the ABAQUS/Explicit version 6.2-1 to analyse the deformation of particles upon impact. Analyses were performed by using an axisymmetric, and a three-dimensional model of double impacts, with various combinations of calculation settings concerning element type, initial and adaptive meshing, contact interaction, etc. Adaptive meshing was used initially to cope 
with large deformations near the contact surfaces. This was done with the objective of preserving initial mesh grading, combined with the built-in second-order advection and half-index shift momentum advection methods of ABAQUS/Explicit. In most cases the frequency of remeshing was kept lower than one in every 50 increments. Based on the modelling results, the bonding of particles can be attributed to adiabatic shear instability which occurs at the particle/substrate or particle/particle interfaces at high velocities. The modelling also shows a very non-uniform development of strain and temperature at the interface, suggesting that this bonding is confined to a fraction of the interacting surfaces.

LI [LI 09b] of Northwestern Polytechnical University (China) conducted an examination of cold spray particle impacting behavior using the ABAQUS/Explicit with a Lagrangian formulation for typical $\mathrm{Cu}$ material (OFHC). Owing to the axisymmetric characteristic of normal impact process, a 2D symmetrical model was used, and the substrate was taken as a cylinder. The radius and height of the substrate were taken to be five times larger than the particle diameter. The particle/substrate interaction was implemented by using the surface-to-surface contact (Explicit) formulation available in ABAQUS. Some important setting factors involving in the simulations - Solution procedure, Hourglass control, Element distortion control, Adaptive meshing, Interfacial friction, Meshing size, Material Damage - were investigated. Through the systematic investigation of the effects of the simulation settings on the output in modeling the impacting behavior of cold spray particles, the feasibility and capability of numerical simulation of particle impacting by the ABAQUS/Explicit program were evaluated. The results showed that the satisfactory output could be obtained with the appropriate settings, but careful regulation of the setting factors is necessary. LI and his team published a series of articles [LI 09a, SHU 09, YIN 10, LI 07a, YIN 11, LI 06] about simulation of cold spray particles deposition process, and this article particularly gives a global and detailed description on modeling techniques.

YILDIRIM [YIL 11] of Northeastern University (United States) carried out a systematic analysis of a single $\mathrm{Cu}$ particle impacting a semi-infinite $\mathrm{Cu}$ substrate for initial impact velocities ranging between $100 \mathrm{~m} / \mathrm{s}$ and $700 \mathrm{~m} / \mathrm{s}$ by using the three different reference frames: Lagrangian reference frame; ALE reference frame; considering material failure in a Lagrangian reference frame. Simulations involving Lagrangian and ALE approaches were done by using a 2D axisymmetric model, where as the simulations involving the Lagrangian approach with material failure was done by using a 3D model with quarter symmetry. The particle and substrate are both assumed to be at room temperature $(293 K)$ prior to impact. Friction coefficient between particle and substrate is taken as 0.3 for all cases. Surface-to-surface contact algorithm is used for the 2D axisymmetric simulations, and the general contact algorithm is used in 3D Lagrangian simulations with material failure. According to his modelling results, the Lagrangian approach with material failure was found effective in describing material behavior under high deformations and preventing excessive distortion of the mesh. Furthermore, it was shown that interfacial outputs such as maximum temperature or maximum plastic strain depend very much on the mesh size. The adaptive remeshing with the ALE approach results in interpolation errors, where decrease in plastic strain in time was observed. 
GHELICHI [GHE 11] of Politecnico di Milano (Italy) developed a totally different 3D numerical model aimed to calculate the critical velocity. The physical bonding has not been modeled and the particles are detached after impacting the substrate. General contact without friction has been regarded as the interaction model between the particle and the substrate. However the deformation of the particles and the consequent substrate profile is quite similar to what happens in real process. The challenging problem of detecting the critical velocity using the discrete output of numerical simulation has been solved applying Wavelet transformation and the second derivative of the physical parameters in Sobolev space.

BAE [BAE 08] of Hanyang University (Korea) divided the different materials into four impact cases according to their physical and mechanical properties, i.e. soft/soft, hard/hard, soft/hard, and hard/soft (particle/substrate). Four-node bilinear axisymmetric quadrilateral mesh elements with reduced integration and hourglass control (CAX4R) from the ABAQUS element library were used, and a surface-to-surface penalty contact algorithm with balanced contact pair formulation was applied between particle and substrate. Individual particle $(25 \mu \mathrm{m})$ impact behaviors of four cases were numerically analyzed and characterized through finely designed micro thermo-mechanical modeling. Based on that analysis, a thermal boost-up zone (TBZ) was theoretically defined and numerically proposed. In the similar cases (soft/soft and hard/hard cases), the ratio between recoverable strain energy and plastic dissipation energy is inversely proportional to the evaluated TBZ at critical velocities. Meanwhile, in the dissimilar cases (soft/hard and hard/soft cases), adiabatic shear instability was always observed at the soft impact counterpart.

OGAWA [MAN 12, MAN 11a] of Tohoku University (Japan) and his research team published a series of articles focusing on the simulation of cold spray particle deposition process using Smoothed Particle Hydrodynamics. In their simulation work, SPH uses the interpolation kernel defined in terms of a cubic B-spline to evaluate these functions by obtaining the kernel estimates. They first introduced adhesion model between the contacting surfaces, by assuming that bonding strengths arise as a result of secondary intermolecular forces at the interface. The interface reaction model was driven by inter-surface traction modeled by the Dugdale-Barenblatt cohesive zone model, and limited to interaction of SPH particles from two different bodies. Meanwhile, this adhesion model was activated upon formation of mutually conforming contact surfaces. The particle deformation behavior modeled by the SPH numerical approach compares fairly well to that modeled by the Eulerian approach which indicates the feasibility of the SPH numerical approach for simulating the impact behavior in cold spraying. The cohesive zone model could be used to describe the particle/substrate interaction correctly.

YIN [YIN 10, LI 10b] of Dalian University of Technology (China) conducted a systematic examination of the normal, oblique and multiple impacts of $\mathrm{Cu}$ particles in cold spraying by using the SPH compared to the Lagrangian, ALE, Eulerian numerical approaches. According to her study, the meshing size and the element type significantly affect the resultant output. Moreover, the particle deformation behaviour calculated by Eulerian formulation is more comparable to the experimental observation than that by 
Lagrangian formulation. Further study on the multi-particle impact process also demonstrates that Eulerian approach is superior to Lagrangian approach. In addition, SPH numerical approach shows that this technique can provide a relatively reasonable result in the particle deformation behavior and the weight of the independent SPH particle exerts limited effects on the resultant output.

SMOJVER [SMO 11] of University of Zagreb (Croatia) predicted the bird strike induced damage in real aeronautical structures using highly detailed finite element models and modern numerical approaches. The problem of soft body (bird) impacts has been tackled by applying the Coupled Eulerian Lagrangian technique. Hydrodynamic response of the bird replacement material is modeled with the equation of state (EOS) materials, which define the volumetric strength of material and pressure to density ratio. The CEL bird model presented in this work has been validated by comparison with Lagrangian bird model and experimental results. He concluded, the improved similarity of impacted plate shape predicted by CEL numerical approach is a direct result of the physically enhanced modeling of fluid-like bird behavior by the CEL bird model. The stability of the analysis is also improved as the CEL bird model does not suffer from significant mesh distortion. The main disadvantage of the CEL model, compared to the Lagrangian bird model, is the much higher computational time due to the need for a very fine mesh of Eulerian elements to accurately simulate the contact with the Lagrangian mesh.

QIU [QIU 11] of Hamburg University of Technology (Germany) investigated the capability of CEL numerical approach on solving the geomechanical problems. The benchmark test of strip footing problem showed that CEL is well suited to deal with problems which cannot be fully solved using FEM. The CEL was applied further to simulate the installation of a pile into subsoil and a ship running aground at an embankment. The comparison between the simulation results and in situ measurement data allows to conclude that the CEL approach is well suited to investigate the influence of pile installation on the surrounding soil or adjacent structures, as well as the interaction between soil and structure with high friction values. In addition, he summarized the quality of the parallelization of the CEL approach was satisfactory.

\subsubsection{Effect of parameters}

Many factors contribute to the formation of cold spray coatings, only a few of them are listed below.

- Powder: Material property, size fractions, morphologies, purity, velocity, temperature, contact angle;

- Substrate: Material property, surface roughness, surface hardness, shape, temperature, native oxide;

- Gas: Type, pressure, temperature;

- Equipment: Nozzle unit, powder feeder, gas heater. 


\subsubsection{Velocity}

The concept of a critical particle velocity for coating formation was developed by Alkhimov, Kosarev, and Papryrin [PAP 06], in the early stages of the process optimization and has been expanded on by various authors. The critical velocity for a given powder is the velocity that an individual particle must attain in order to deposit (or adhere) after impact with the substrate. Generally the critical velocity will be within a range of velocities, since the starting powder feed stock is usually a distribution of particles sizes. Below the range region the particles do not form coatings, and above the range region coatings rapidly build to millimeter or thicker dimensions. Particle velocity is influenced by several factors: main gas temperature, molecular weight of the main gas, main gas pressure, powder carrier gas effects, particle size, residence time in the high-velocity gas flow, particle shape, particle density, and the interaction with the bow shock wave at the substrate surface to name a few.

The relationship that governs the particle velocity inside the nozzle is the coupling of the gas velocity to the particle drag in the gas stream. The equation for the speed of sound $v$ is:

$$
v=\left(\gamma R T / M_{w}\right)^{1 / 2}
$$

where $\gamma$ is the ratio of specific heats (1.4 for air, 1.66 for $\mathrm{He}$ ), $R$ is the gas constant (8314 J/kmolK), $T$ is gas temperature, and $M_{w}$ is the molecular weight of the gas. A convenient way to increase particle velocity (i.e. increase the gas velocity) is to increase the main gas temperature or use a smaller molecular weight gas such as helium.

FIG.1.8 is a one-dimensional computation of the velocities for the main gas and the particles assuming air as the main gas, inlet gas temperature of $527^{\circ} \mathrm{C}$, inlet pressure to be 2.0 $\mathrm{MPa}$, and $\mathrm{Cu}$ as the coating powder [STE 99]. From this figure, one observes that for particles less than $5 \mu \mathrm{m}$, the velocities increase rapidly after passing through the throat, while for larger particles $(>5 \mu \mathrm{m})$ they travel at lower velocities. It is this interaction between the particle and the high velocity gas that allows one to tailor the different spray processes for coating formation.

\subsubsection{Temperature}

The dependence on a critical velocity for coating formation is only one of the critical aspects used to determine the coating formation. Equally important is the particle temperature. The temperature of the gas stream is always below the melting point of the particulate material during cold spray, and the resultant coating and/or freestanding structure is formed in the solid state. Since adhesion of the metal powder to the substrate, as well as the cohesion of the deposited material, is accomplished in the solid state, the characteristics of the cold spray deposit are quite unique in many regards. FIG.1.9 shows the heating of the particles as a function of size and distance from the throat. One notes that the smaller particles $(<5 \mu m)$ has a similar response to that of gas, i.e.particle cooling 


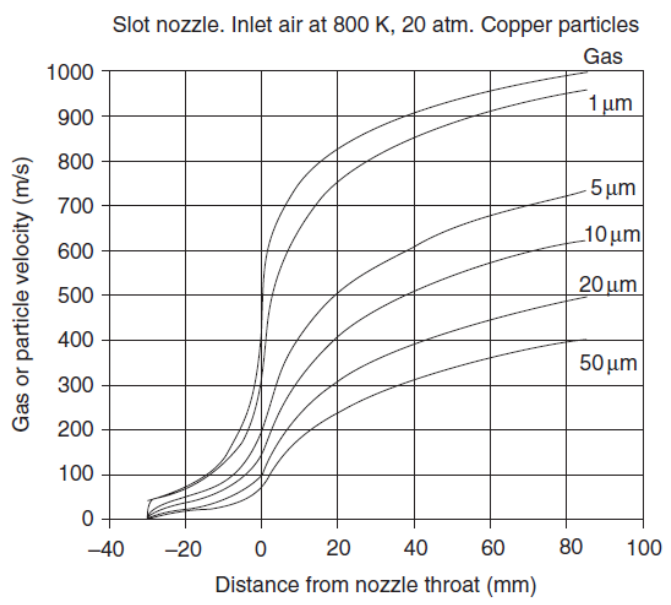

Figure 1.8: Computed air and particle velocities as function of distance along the nozzle [STE 99].

during the trip through the diverging section of the nozzle [STE 99]. Larger particles with lower velocities, however, have higher temperature compared to the smaller ones.

The temperature influences the deposition process of cold spray in different manners, and its influence are summarized in TAB.1.1 by considering the outcomes of experiments [KLI 05]. First, with increasing stagnation temperature the velocity of the process gas is increased, and accordingly also the impact velocity of particles. Once the particles attain sufficient kinetic energy to be transformed into heat energy and strain energy, the coating will be produced successfully layer by layer. Second, it is known, that elastic and plastic properties of materials depend on the temperature. The temperature of the materials can be changed both, by using a higher gas temperature and by preheating the substrate. An increased temperature of the materials could enhance thermal softening which is important for the bonding mechanism.

Velocity and temperature, these two parameters mainly determine whether a particle will produce a coating or not. The particle velocity will determine the amount of kinetic energy available for plastic deformation. The particle temperature will determine the mechanical properties of that particle at the moment of impact. Increasing the particle temperature generally results in a lowering of the critical velocity, while decreasing the particle temperature will generally result in a need for higher particle velocity.

\begin{tabular}{|c|c|c|c|c|}
\hline Case & Gas & Gas heating & Substrate heating & Attachment \\
\hline 1 & air & no & no & no \\
2 & air & yes & no & yes \\
3 & air & no & yes & yes \\
4 & air+helium & no & no & yes \\
\hline
\end{tabular}

Table 1.1: Influence of temperature on the deposition of $\mathrm{Al}$ particles impinging with high velocities onto substrates of $\mathrm{Cu}$ and steel [KLI 05]. 
Slot nozzle. Inlet air at 800 K, 20 atm. Copper particles

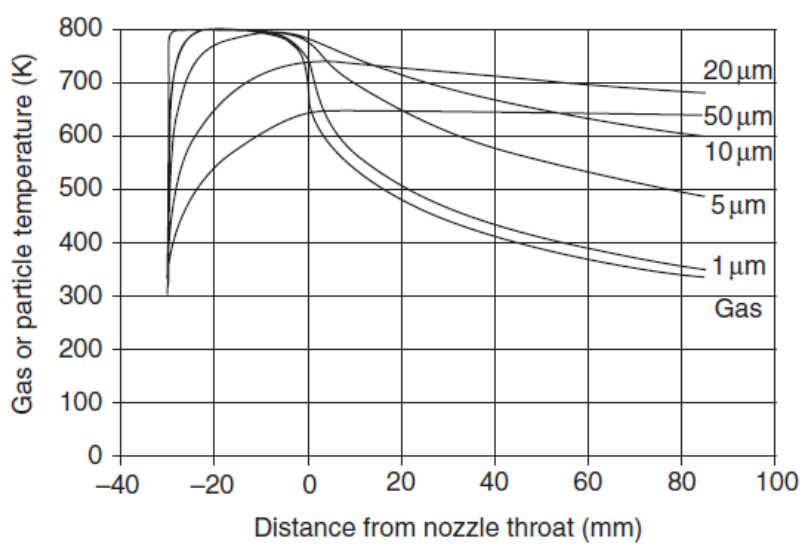

Figure 1.9: Computed air and particle temperatures as function of distance along the nozzle [STE 99].

\subsubsection{Contact angle}

Most investigations on deposition behavior in cold spraying were focused on the normal impacts. Practically, owing to the profile of the substrate and even surface roughness, spray particles may impact at an off-normal angle with respect to the substrate surface. GUETTA et al. [GUE 09] suggested that particle adhesion mechanisms are influenced by particle position. Different splat morphologies [GUE 09] observed by SEM as a function of positions from the center of the particle jet for copper particles with diameter of $10 \mu \mathrm{m}$ is shown in the FIG.1.10. The deformation shape of particle near the rim of particles flow is significant different from that of particles at the center of particles flow. Past researches both on experiments [PAT 08] and simulations [SAM 07, LEE 11] have proven that the strong bow shock was formed on the substrate, a high stagnation pressure zone thus was created near the substrate surface. This high pressure region will result in an off-normal impact on the substrate. The deviation of particle trajectories from the center line can be so strong that some particles may not impact the substrate and are washed away by the gas flow, causing the erosion of substrate under low impact angle circumstances. These phenomena are found to be directly responsible to reduce the process deposition efficiency. Naturally, the normal component of particle velocity will be less than that of vertical impact. The particle and substrate present an asymmetric deformation under oblique impact condition compared with that in normal impact. It was confirmed that the normal component of particle velocity contributes essentially to the deposition of the particle under the off-normal spray condition, while the tangential component of the velocity causes the particle to move along the substrate surface [LI 07a]. The deposition efficiency of particles is decreased owing to the decrease of the normal velocity component [LI 05]. FIG.1.11 shows schematically the change of the relative deposition efficiency with off-normal contact angle, assuming that the minimum particle velocity is higher than the critical velocity at a given spray condition at normal angle. The contact angle can be dived into three typical regions: maximum deposition region, transient region and free of deposition re- 
gion. The maximum deposition region is around the vertical direction and its deposition efficiency reaches nearly $100 \%$. On the contrast, the surface free of deposition extends from zero degree to the critical angle, where the erosion of substrate surface may occur. In the transient region, the deposition efficiency increases from $0 \%$ to $100 \%$, depending on the velocity of the particles.
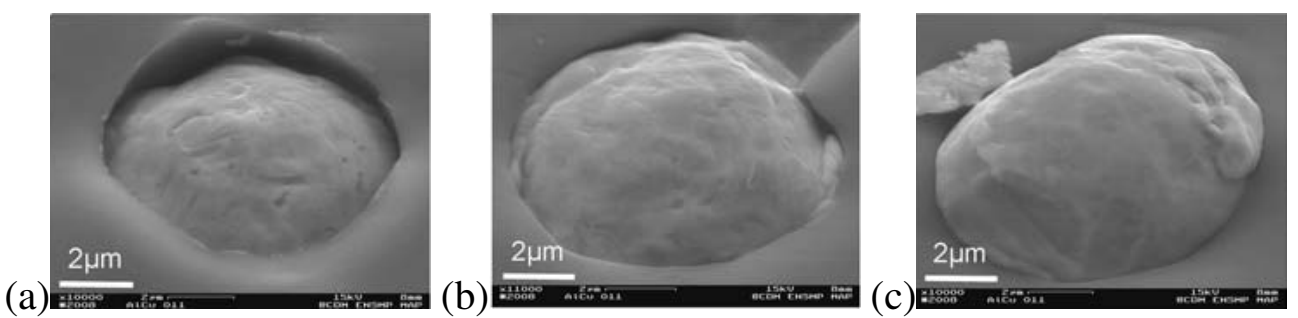

Figure 1.10: Different splat morphologies observed by SEM as a function of positions from the center of the particle jet: (a) near the center, (b) intermediate position and (c) near the rim.

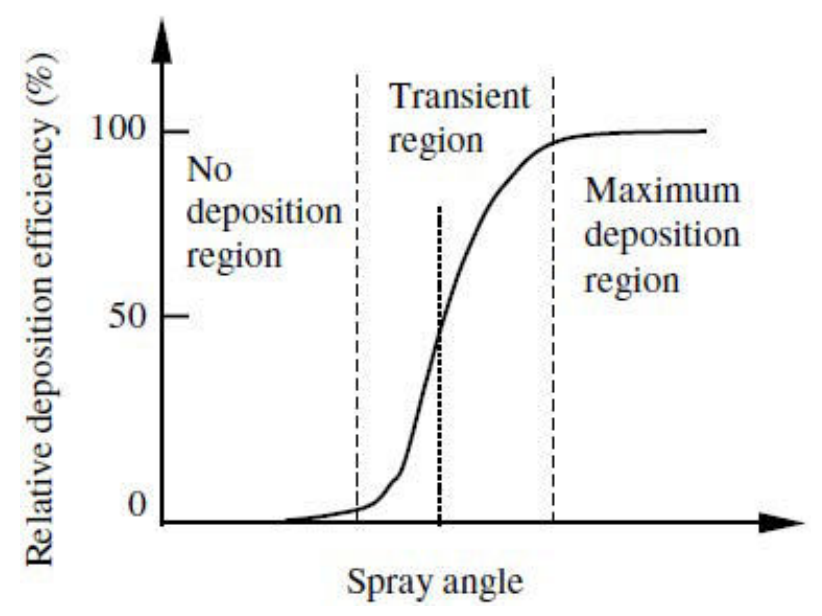

Figure 1.11: Schematic diagram of the dependence of relative deposition efficiency on spray angle [LI 05].

\subsubsection{Experimental results}

\subsubsection{Porosity rate}

Although deposition is successful, inherent porosity limits utilization of the cold spray process in many industrial applications that demand high density, high thermal conductivity, and postspray machining (e.g., mirror-finish surface quality). Particle velocity [VEN 11, CHA 11], particle temperature [ZAH 06, CHA 11, SUD 07], and particle size distribution [ZAH 06, ZAH 09] are the most important processing conditions that affect porosity formation in the coatings. Other deposition parameters, like gas type [ZAH 09], 
pressure [VEN 11, CHA 11] and stand-off distance [ZAH 09, SUD 07] and vacuum treatment [SUD 07], also have influences on the elimination of porosity of coatings.

The etched micrographs of the silver coatings [CHA 11] obtained at particle velocities of $363 \mathrm{~m} / \mathrm{s}, 403 \mathrm{~m} / \mathrm{s}$ and $469 \mathrm{~m} / \mathrm{s}$, foremost indicate the presence of large unbonded areas between adjacent powder particles in coating obtained at $363 \mathrm{~m} / \mathrm{s}$ (FIG.1.12a) unlike in the case of coatings deposited at higher velocities (FIG.1.12b and c). A careful comparison of the splat morphologies in FIG.1.12 also brings out the fact that the deformation of the particles during cold spraying increases with increasing particle velocity as indicated by the increasing aspect ratio of the splats with increasing velocity. The decline in volume fraction of the porosities is most likely due to this large plastic deformation of the particles.

Microstructural observations [ZAH 06] show that an increase in temperature led to a decrease in volume fraction of the porosity in the coating (FIG.1.13). Quantitative image analysis of the micrographs in FIG.1.13 (at least 10 images for each temperature) indicates a decrease in volume fraction of porosity from $0.7 \%$ at $300{ }^{\circ} \mathrm{C}$ to $0.05 \%$ at $420{ }^{\circ} \mathrm{C}$ and $0.005 \%$ at $630{ }^{\circ} \mathrm{C}$. Meanwhile, the average porosity size declined from $45 \mu \mathrm{m}$ at $300{ }^{\circ} \mathrm{C}$ to $2 \mu \mathrm{m}$ at $630{ }^{\circ} \mathrm{C}$. This confirms a larger deformation for particles at high temperatures that results in a decrease in size of the voids and cavities in the coating.

A schematic representation of the particle size distribution effect on porosity formation is presented in FIG.1.14. Three powders are presented: (1) a powder with a small particle size and a narrow particle size distribution; (2) a powder with a mixture of small and large particles that represents a broad particle size distribution; and (3) a large particle size powder with a narrow particle size distribution. In the ideal case (1), small particles achieve the maximum velocity required for particles to significantly deform and eliminate voids between particles. Under the conditions that a mixture of large and small particles deposit, case (2), small particles acquire enough velocity (kinetic energy) to deform significantly. However, the large particles do not considerably deform. This results in a heterogeneous porosity formation with small and large cavities. In the last case (3), large particles may achieve the critical velocity for deposition but with limited plastic deformation. Therefore, the cold spray coating exhibits porosities in the microstructure that may develop due to interparticle gaps and bridging of the particles [ZAH 06].

Besides, experiment results show that using helium as the deposition gas has a significant influence on the elimination of pores, and decreasing pressure from 3 to $2 \mathrm{MPa}$ led to an increase in volume fraction of porosity by more than two orders of magnitude from 0.005 to $1.1 \%$. The volume fraction of porosity decreased with a decrease in the distance between the nozzle and the substrate.

\subsubsection{Residual stress}

Typically, all these three components will be present in the conventional thermal sprayed coatings, which are quenching, thermal and peening stresses.

- Quenching stress The temperature drop in the solidification of molten particles upon impact with the substrate leads to quenching stresses because the contraction 
(a)

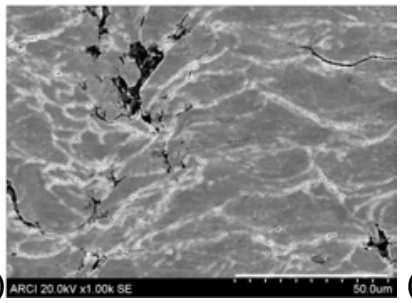

(b)

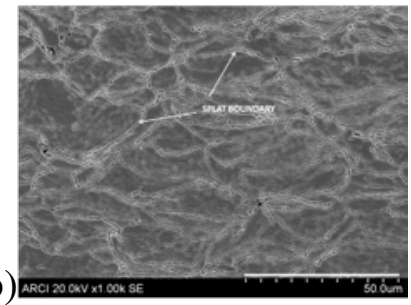

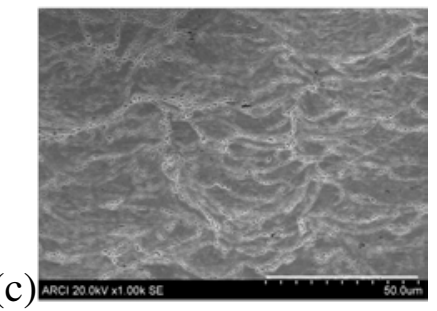

Figure 1.12: Etched Cross section micrographs of coatings obtained at a particle velocity of (a) $363 \mathrm{~m} / \mathrm{s}$ (b) $403 \mathrm{~m} / \mathrm{s}$ and (c) $469 \mathrm{~m} / \mathrm{s}$ [CHA 11].
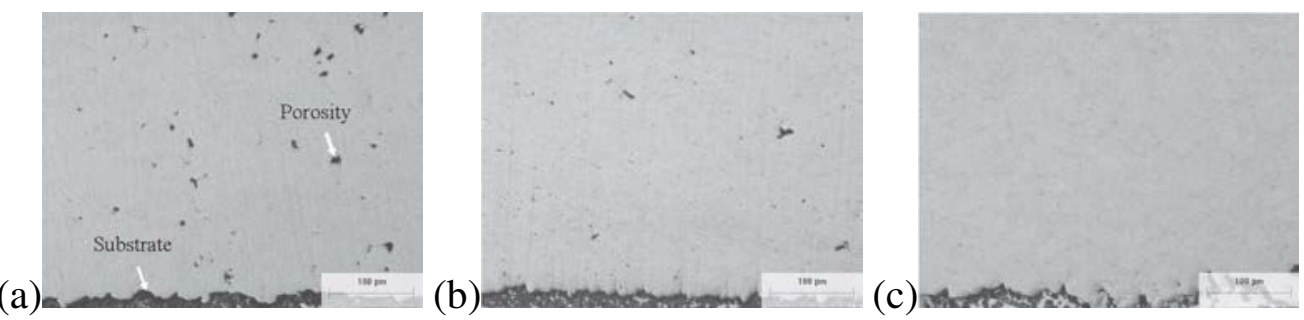

Figure 1.13: Porosity formation in cold sprayed microstructure for gas temperatures of (a) $300{ }^{\circ} \mathrm{C}$, (b) $420{ }^{\circ} \mathrm{C}$ and (c) $630{ }^{\circ} \mathrm{C}$ [ZAH 06].

is restricted by the adherence to the substrate.

- Thermal stress When the substrate-coating system is cooled down to ambient temperature after the deposition, a thermal mismatch stress is generated. The nature of the thermal stress (tensile, neutral or compressive) is determined by the difference in the thermal expansion coefficients $(\triangle \mathrm{CTE})$ of the coating and the substrate.

- Peening stress In high velocity cold spray systems, a peening stress can be introduced during the deposition due to plastic deformation of the substrate and/or previously deposited coating material.

The combined effect of quenching and peening is known as evolving stress, and represents a stress developed when a layer of material is deposited in the substrate-coating system. Compressive evolving stress indicates that the peening effect is more dominant, and the tensile mode indicates that the quenching effect is more dominant. The magnitude of the quenching, thermal and peening stress is dependent on [SUH 13]:

- The temperature difference before and after the impact of the particle with the substrate;

- The temperature difference of the substrate-coating system during and after the spraying;

- The ability of the substrate and coating to plastically deform;

- The working harden upon impact of the particle materials.

The thermal stress may cause peeling and delamination of coating. This problem is compounded when the substrate material is different from the coating material, however, it could be minimized or eliminated when cold spray is used. Nevertheless, a considerable compressive stress arises during the cold spray deposition process. A state of compressive 


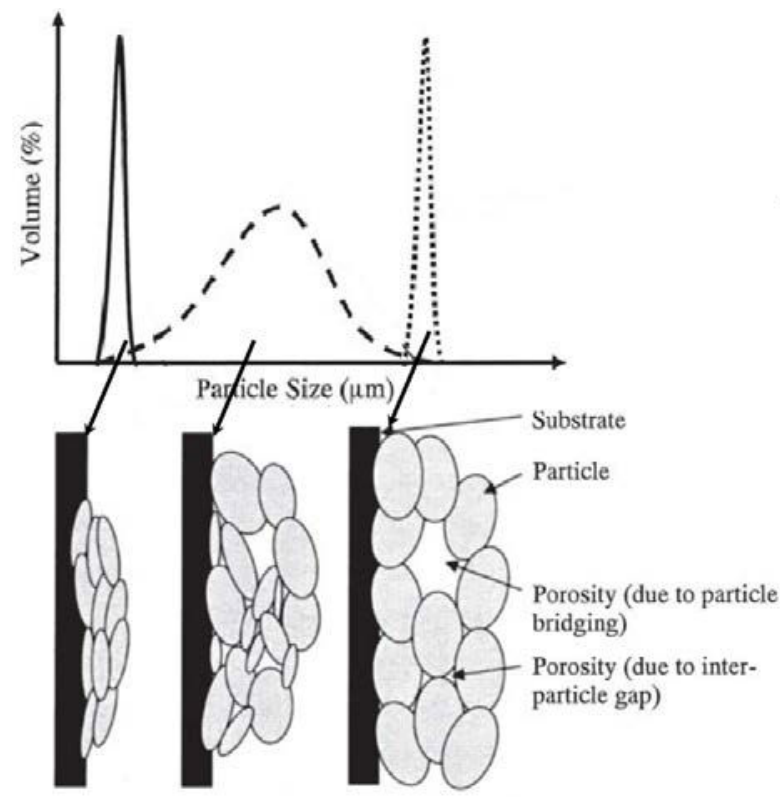

Figure 1.14: Effect of particle size distribution on porosity formation in cold spray process [ZAH 06].

residual stress on the surface is generally thought to be beneficial for fatigue resistance.

Several methods including theoretical models and experimental methods have been used for residual stress measurement and estimation of cold spray coatings.

TSUI and CLYNE [TSU 97a, TSU 97b, TSU 97c] developed a model to predict the residual stress distribution in progressively deposited thermal spray coatings. This is based on the concept of a misfit strain, caused by either the deposition stress (e.g. due to quenching of splats accompanying the rapid solidification and cooling process of droplets coalescing on the developing layer in thermal spraying) or by differential thermal contraction between substrate and coating during cooling.

MCCUNE [MCC 00] determined the composite residual stress in the coating layers and substrate by using the modified layer removal technique. For these determinations, the as-deposited layers were mechanically ground to produce a planar surface, free of any waviness associated with irregularities of the deposition and having a final thickness of approximately $2 \mathrm{~mm}$. The residual stress distribution as a function of depth from the planarized outer surface was measured for $\mathrm{Cu} / \mathrm{Cu}$ coating system displayed in FIG.1.15. The deposit layer produced from direct-reduction $\mathrm{Cu}$ showed a characteristic compressive region in the surface of the substrate with a maximum compressive stress of approximately $60 \mathrm{MPa}$ near the substrate interface. In the case of the gas-atomized $\mathrm{Cu}$ compact, the interfacial compressive region extended into the coating to a greater extent with a maximum compressive stress adjacent to the interface of approximately $120 \mathrm{MPa}$.

LUZIN [LUZ 11] used the neutron diffraction method to study the in-plane residual stress profiles of $\mathrm{Cu}$ and $\mathrm{Al}$ cold spray coatings as shown in FIG.1.16. Both $\mathrm{Cu}$ coatings show a significant compressive residual stress at the surface around 50-80 $\mathrm{MPa}$, while the 
surface residual stress of the $\mathrm{Al}$ coatings is less than $10 \mathrm{MPa}$. The overall stress profile of the two $\mathrm{Cu}$ coatings is similar, regardless of the substrate material, and the same as $\mathrm{Al}$ coatings.

GHELICHI [GHE 12] studied the state of residual stresses using XRD analysis. It was performed using an AST X-Stress $3000 \mathrm{X}$-ray diffractometer $\mathrm{Cr}$ Ka radiation, irradiated area of $1 \mathrm{~mm}$ diameter, $\sin ^{2}(\Psi)$ method, diffraction angle $(2 \theta)$ of $139^{\circ}$ corresponding to lattice plane (311) scanned between $-45^{\circ}$ and $45^{\circ}$ ). Residual stress results for the as-received specimens coated with Al7075 and pure Al are presented in FIG.1.17. Compressive residual stress in the interface between the substrate and the deposited material can be observed in both cases.

SUHONEN [SUH 13] firstly introduced a curvature-based approach, where the substrate-coating system deflection and temperature are monitored throughout the coating deposition process, to measure the in situ residual stress of several cold spray materials. Typical ICP-sensor (an in situ coating property sensor) curvature and temperature curves with explanations of the stress component are presented in FIG.1.18. The evolving and deposition stresses were monitored from the start to the end of the actual coating deposition, the thermal stresses from the end of the deposition to the cooling down of the coating-substrate system to room temperature, and the final residual stress state was given as the sum of the stresses generated.

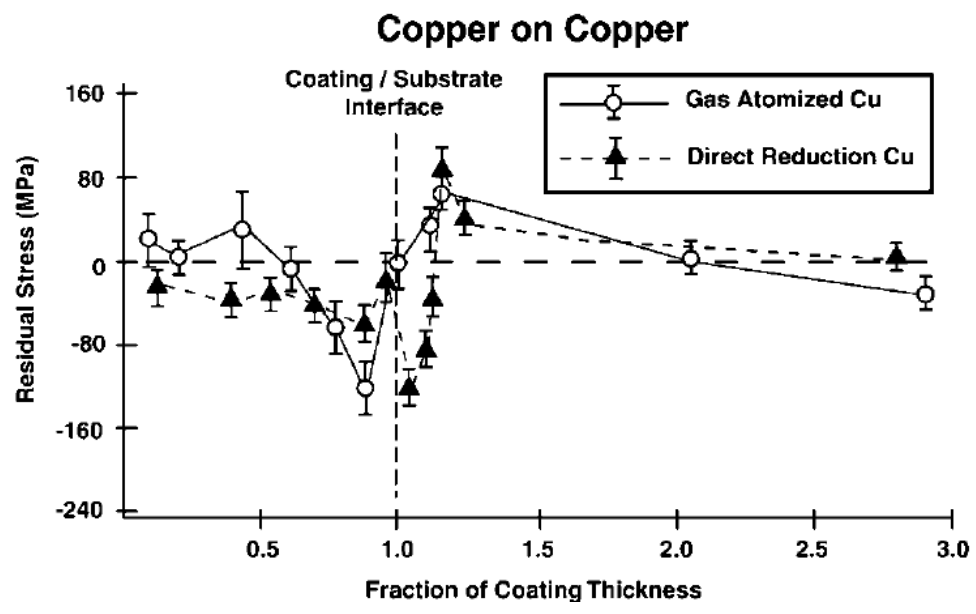

Figure 1.15: Comparison of through-thickness residual stress distributions measured by the modified layer-removal technique for compacts produced from gas atomized and direct-reduction $\mathrm{Cu}$ powders [MCC 00].

\subsubsection{Erosion damage}

HUTCHINGS defined erosion as 'an abrasive wear process in which the repeated impact of small particles entrained in a moving fluid against a surface results in the removal of material from that surface' [WAN 08]. For ductile materials such as metals and alloys, materials removal is caused by the micro-cutting and micro-ploughing of the solid parti- 
(a)

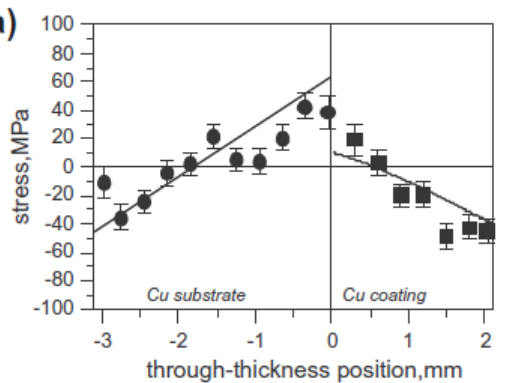

(c)

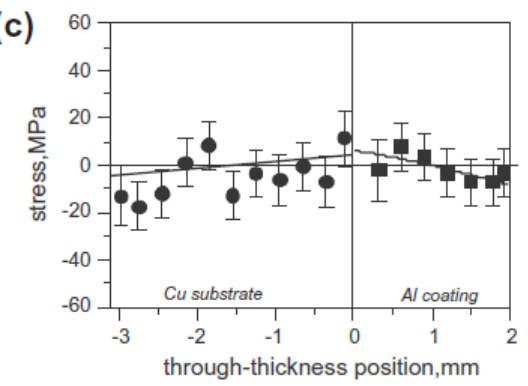

(b)

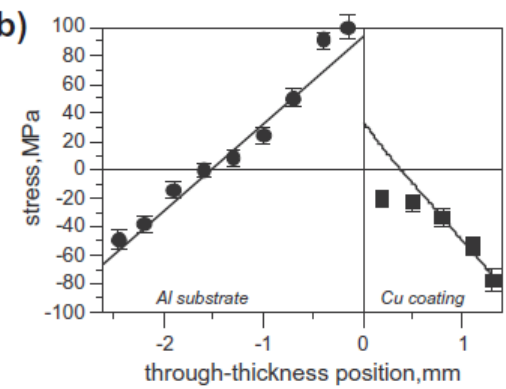

(d)

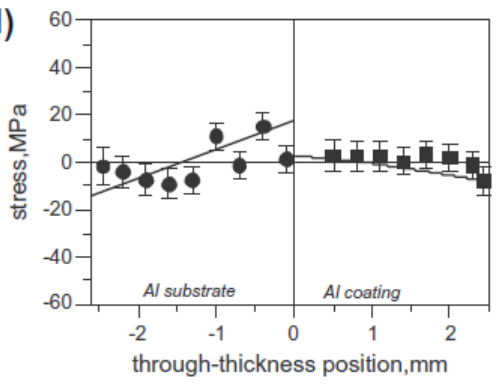

Figure 1.16: Measurement (symbols) and model fit (solid lines) of the through thickness in-plane stress distributions for (a) $\mathrm{Cu} / \mathrm{Cu}$ sample, (b) $\mathrm{Cu} / \mathrm{Al}$ sample, (c) $\mathrm{Al} / \mathrm{Cu}$ sample and (d) $\mathrm{Al} / \mathrm{Al}$ sample [LUZ 11].

cles seen in FIG.1.19(a). For brittle materials such as ceramics, the energy transfer from solid particles to the surface of the target materials. This process will induce the material deformation, crack initiation and propagation, materials pieces detachment as FIG.1.19(b) shows. The corresponding experiment results are shown in FIG.1.20.

It was found that wear rate depends mainly on the velocity of erodent particles and on the angle between the target surface and particle trajectory (contact angle). Further, it was revealed how other parameters (i.e. particle size and shape, their concentration, abrasive to target hardness ratio and the effect of liquid and solid additives) can change the nature and result of the process of erosion.

A lot of researches about the erosive wear have been done by Lebedev, Finnie [FIN 60], Bitter [BIT 63], Nepomnyashchy, Abramov, Peter, Beckmann and Gotzmann [KLE 07]. In Ref [KLE 07], the author pointed out that is the Beckmann's and Gotzmann's theory that provides the best possible coincidence with the experimental data. The theory [BEC 81] assumes that before removing the wear product from the metal surface, a certain amount of energy necessary for shear deformation on the surface must be accumulated. Micrographs taken by HUTCHINGS et al. [HUT 76] provide a vivid illustration of the shear deformation on erosion (FIG.1.21(b)).

OKA [OKA 93, OKA 03, OKA 05a, OKA 05c, OKA 05b, OKA 09] conducts a series of experimental study on the erosion damage since the 90s of 20th century. He found that the impact angle dependence was roughly consistent with the maximum principal shearing strain and erosion damage data. The surface topography of the impact craters suggested that depth, contact area and volume of indentation are affected by the particle 


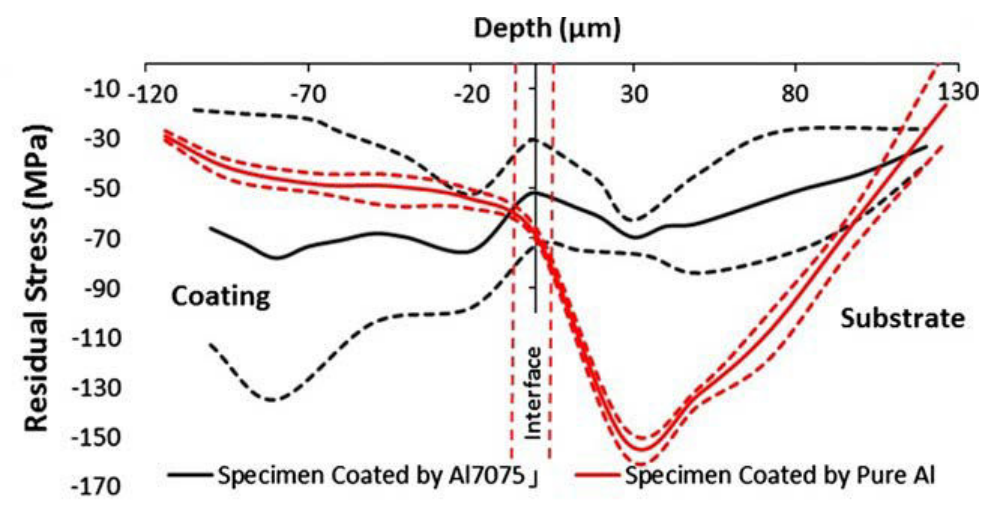

Figure 1.17: Residual stress in as-received specimens coated with Al7075 and pure Al [GHE 12].

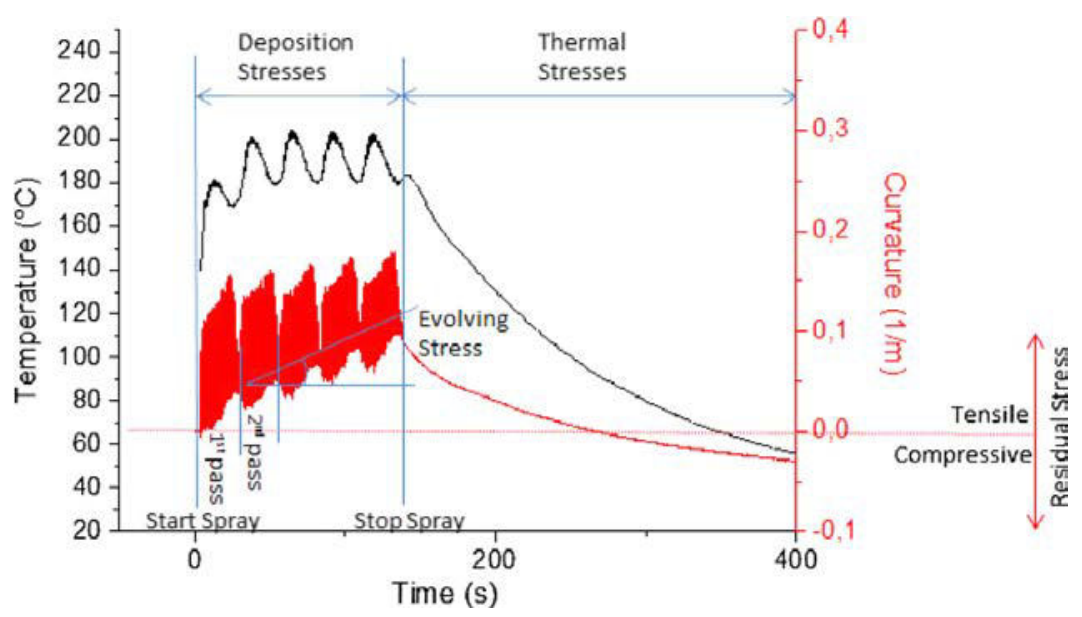

Figure 1.18: Temperature and curvature graphs of Ti/Carbon steel coating, illustrating the concepts of evolving, deposition, thermal and final residual stresses [SUH 13].

density and the hardness of both particle and target material. It is concluded that the origin of erosion is probably attributed to the conjoint actions of high plastic strains followed by subsequent removal and the cutting process caused by particle impact. FIG.1.22 shows SEM observations for craters of Aluminum obtained by the impact tests of WC balls at impact angles of $20^{\circ}, 30^{\circ}, 40^{\circ}, 60^{\circ}$ and $90^{\circ}$ at an impact velocity of $100 \mathrm{~m} / \mathrm{s}$. Considering the damage behaviour caused by hypervelocity particle impact, he concludes that a single particle impact could remove large material with a lower contact angle at a hypervelocity, as illustrate in FIG.1.23. Based on the impact velocity and diameter ration, which is defined as indentation diameter divided by the particle diameter, he proposes three types of crater shapes as shown in FIG.1.24. The crater in Type I is simply formed along the round projectile until the half of the projectile is embedded in the material. The crater in Type II is formed deeply into the material with the same diameter as the projectile, as the impact velocity increases. The crater in Type III exhibits the complicated cross-sectional shape because of the fracture of the projectile. 


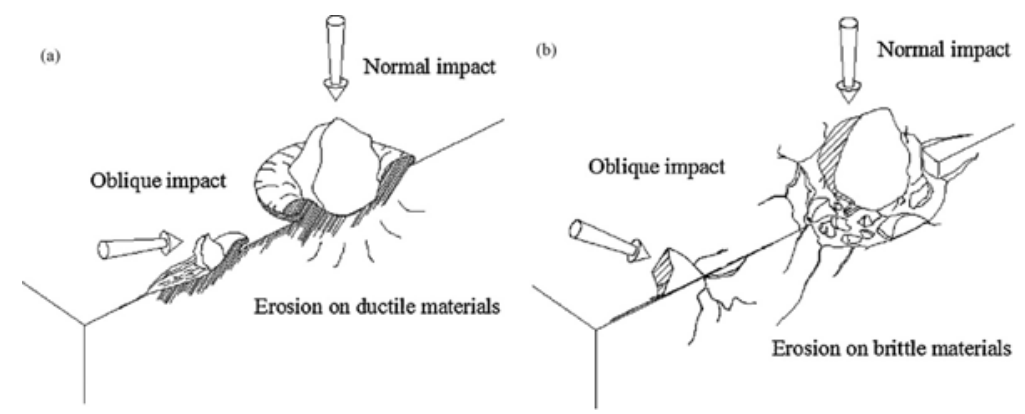

Figure 1.19: The erosive failure behavior of brittle and ductile materials: (a) erosion on brittle materials; (b) erosion on ductile materials [WAN 08].

(a)

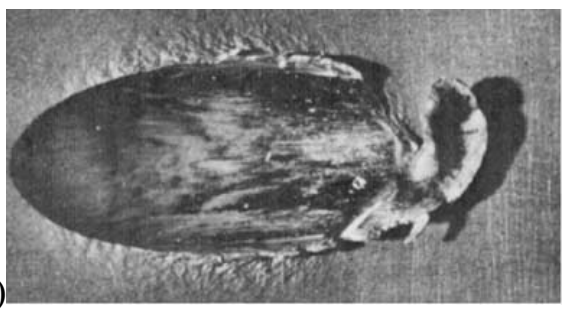

(b)

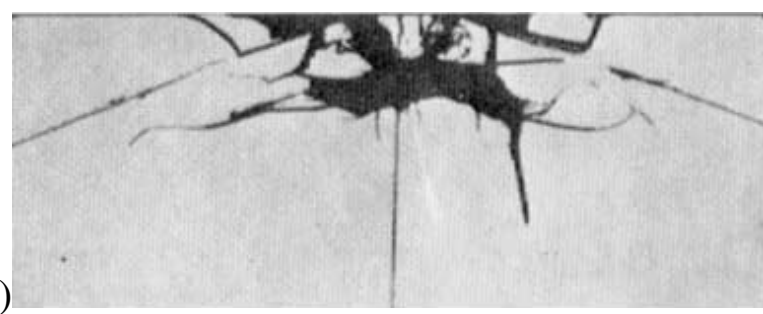

Figure 1.20: Crater shape in (a) ductile and (b) brittle materials [RUF 79, AQU 01].

AKBARZADEH [AKB 12] gives a global view of the erosion phenomenon using magnetite material. He investigates 12 metals with two different particle sizes $(6.9 \mu \mathrm{m}$ and $30.4 \mu \mathrm{m}$ ), two different velocities $(90 \mathrm{~m} / \mathrm{s}$ and $130 \mathrm{~m} / \mathrm{s}$ ), and six different impingement angles $\left(15^{\circ}, 30^{\circ}, 45^{\circ}, 60^{\circ}, 75^{\circ}\right.$ and $\left.90^{\circ}\right)$. Most of the tested materials exhibited ductile erosive behaviour, with maximum erosion rates occurring at shallow impingement angles between $15^{\circ}$ and $30^{\circ}$. The erosion rate depends on the kinetic energy of the particles. The most erosion resistant material was found to be the tungsten carbide (WC), and the least erosion resistant materials were nickel plated A1018 carbon steel. An interesting observation is that the erosion rates for the $6.9 \mu \mathrm{m}$ powder were between 1.7 and 1.8
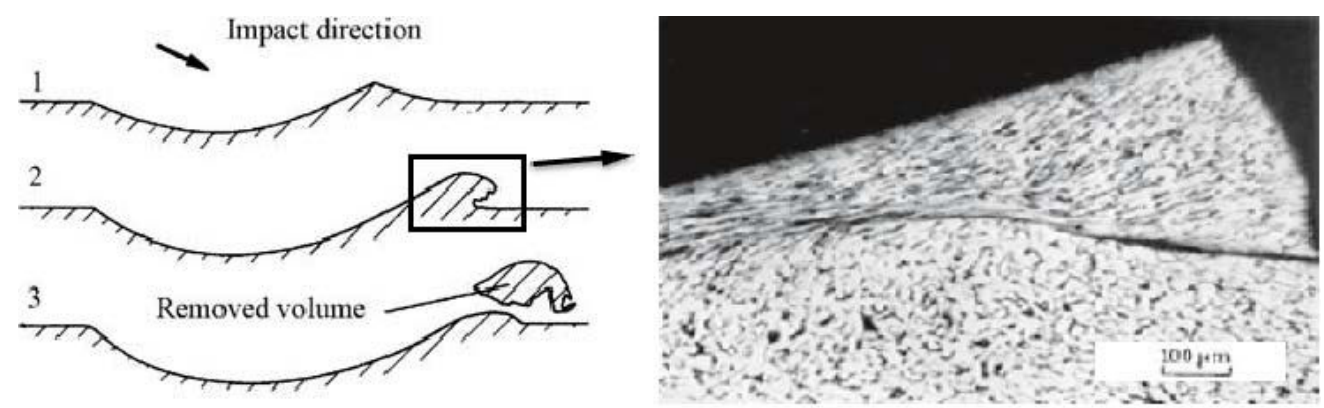

Figure 1.21: Longitudinal section of the impact crater left in mild steel when hit by hardened steel ball $\mathrm{R}=4.75 \mathrm{~mm}, \alpha=30^{\circ}, v_{0}=141 \mathrm{~m} / \mathrm{s}:$ (a) material removal, (b) microphoto of special zone in the black rectangle indicated at (a) [HUT 76]. 


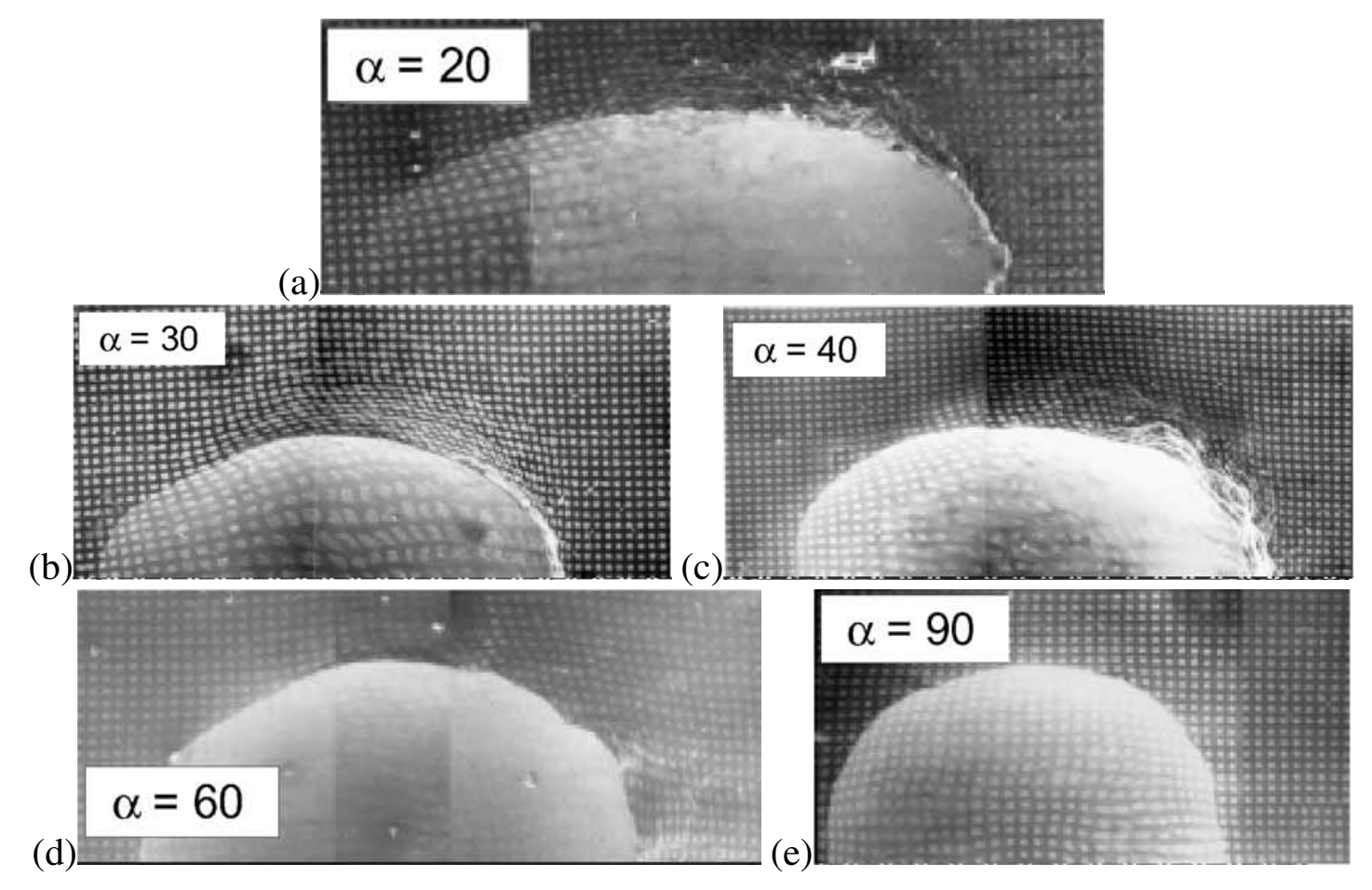

Figure 1.22: SEM observations for craters of Aluminum obtained by the impact tests of WC balls at impact angles of (a) $20^{\circ}$, (b) $30^{\circ}$, (c) $40^{\circ}$, (d) $60^{\circ}$ and (e) $90^{\circ}$ at an impact velocity of $100 \mathrm{~m} / \mathrm{s}$ [OKA 03 ].

times higher than the corresponding rates for the $30.4 \mu \mathrm{m}$ powder. He explains this could be attributed to the greater surface hardening during blasting with the larger particles. Some scanning electron micrographs of erosion scars are displayed in FIG.1.25.

\subsection{Objectives}

The objective of this study is to investigate the deposition process of cold sprayed particle onto the substrate through numerical methods. In chapter 2 , the cold spray deposition process is simulated by modeling the impact of spherical Aluminum particle onto Aluminum substrate using four numerical approaches descried above. In this chapter, the aim is to explore the feasibility and reliability of each numerical approach when solving the high speed impact problem. Thus the details of four numerical approaches are outlined and the capability of each approach is summarized in the last section of chapter 2. Understanding the bonding mechanism between the particle and substrate is the principal goal of chapter 3. Successful bonding depends on several key parameters, their effects on the contact between particle and substrate are investigated by using a single impact model. Based on the simulation results of single particle impact, the multi-particle impact is also simulated using Coupled Eulerian Lagrangian numerical approach in chapter 4. This is the preliminary step for simulating the coating formation process. The impor- 


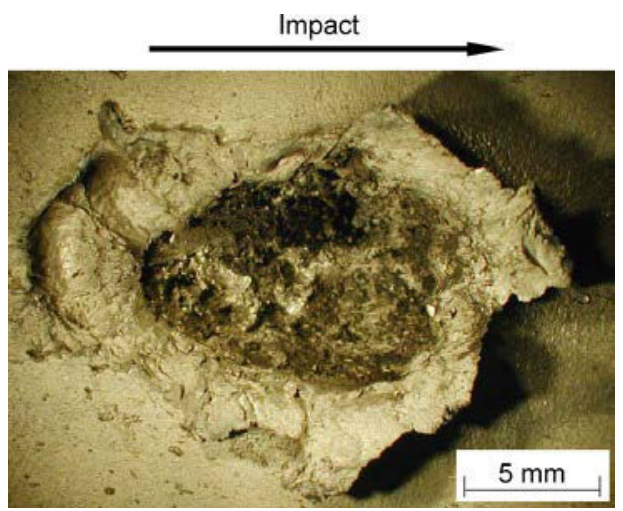

Figure 1.23: A photograph of a crater caused by WC ball impact at an impact velocity of $2500 \mathrm{~m} / \mathrm{s}$ at $30^{\circ}$ for Aluminium [OKA 05c].

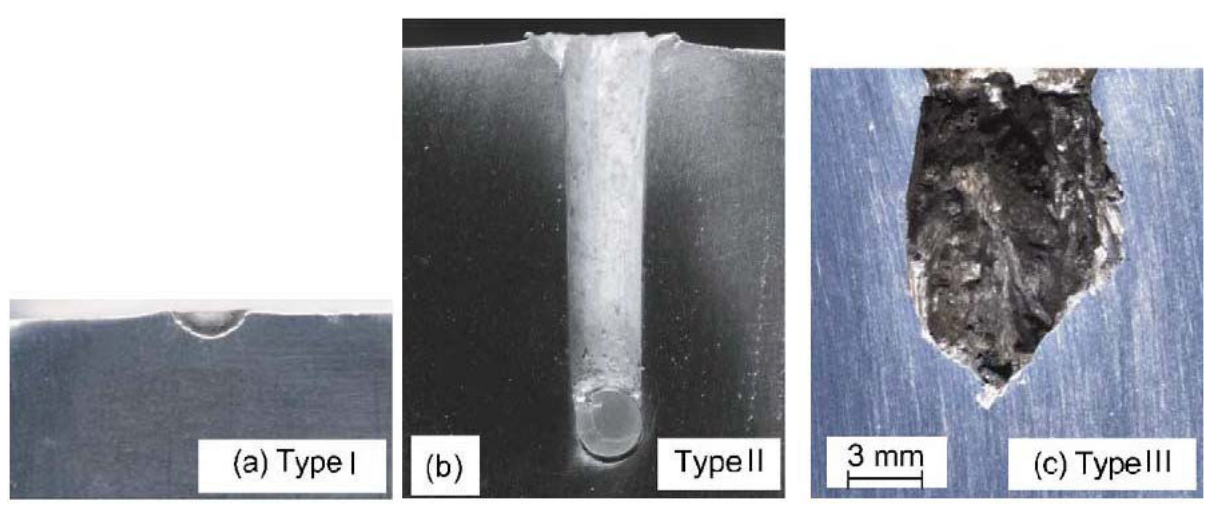

Figure 1.24: Cross-sectional surface of Aluminium at impact velocities of (a) $100 \mathrm{~m} / \mathrm{s}$, (b) $1320 \mathrm{~m} / \mathrm{s}$ and (c) $2270 \mathrm{~m} / \mathrm{s}$ [OKA 05c].

tant object of chapter 4 is to estimate and analyze the porosity rate and internal residual stress within the coating, which affect the coating performances. One of the applications is the simulation of erosion, if the material model combined with material damage model, which is Johnson-Cook damage model in our case. In fact, this phenomenon could possibly happen under the extreme cold spray situation of high velocity or low contact angle impact. 

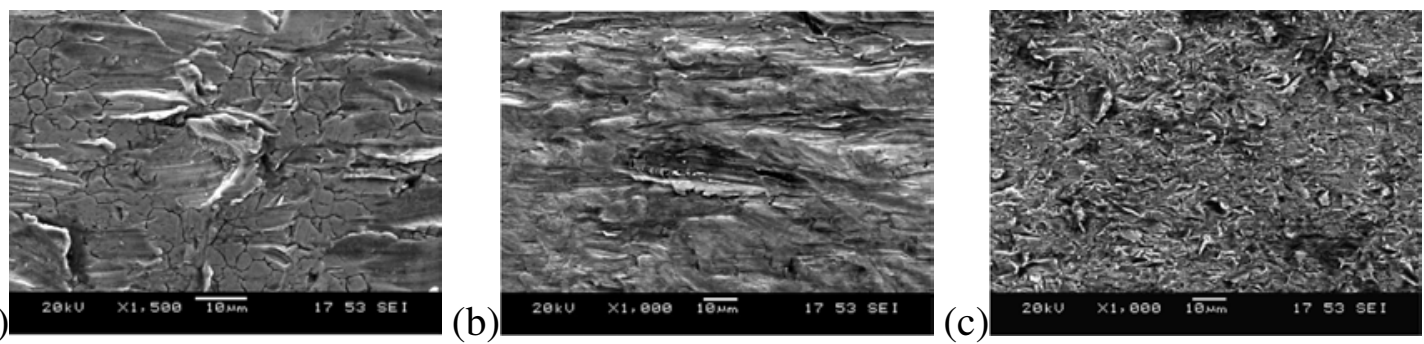

Figure 1.25: Scanning electron micrograph taken from periphery of erosion scar showing ductile erosion mechanisms for (a) 316 stainless steel eroded at $90 \mathrm{~m} / \mathrm{s}$ for a dwell time of $180 \mathrm{~s}, 30.4 \mu \mathrm{m}$ powder at $15^{\circ}$; (b) Stellite 12 eroded at $130 \mathrm{~m} / \mathrm{s}$ for a dwell time of 120 $s, 30.4 \mu \mathrm{m}$ powder at $15^{\circ}$; (c) 316 stainless steel eroded at $130 \mathrm{~m} / \mathrm{s}$ for a dwell time of 180 $s, 6.9 \mu m$ powder at $15^{\circ}$ [AKB 12]. 


\section{Chapter 2}

\section{Feasibility of numerical approach}

This chapter starts with the basic assumptions for modeling the cold spray particle impact on the substrate. Some aspects

involved in these four numerical approaches for contact/impact problem, including the model dimension, material model, analysis procedure, contact algorithm, boundary condition and mesh, are discussed in detail subsequently. The simulation results of deformation behavior of material, and the outputs of stress, strain, temperature, indentation and energy, are analyzed in detail. This chapter concludes with the comparison of four numerical approaches for illustrating their computation capabilities and costs, and a discussion about the typical impact behaviors of cold sprayed particle and substrate.

\section{Contents}

2.1 Problem description . . . . . . . . . . . . . . . . 27

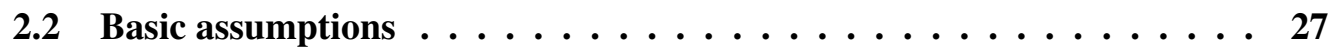

2.3 Lagrangian ....................... 28

2.3.1 Model description . . . . . . . . . . . . . . . . . 28

2.3.2 Results and discussion . . . . . . . . . . . . . . . . 34

2.4 Arbitrary Lagrangian Eulerian . . . . . . . . . . . . . . . 36

2.4 .1 Model description $\ldots \ldots \ldots \ldots$ 
2.4 .2 Results and discussion $\ldots \ldots \ldots \ldots$. . . . . . . . . . 38

2.5 Smoothed Particle Hydrodynamics . . . . . . . . . . . . . . . . 40

2.5.1 Model description . . . . . . . . . . . . . . . . . . . 40

2.5.2 Results and discussion . . . . . . . . . . . . . . . . . 43

2.6 Coupled Eulerian Lagrangian $\ldots \ldots \ldots$. . . . . . . . . . 44

2.6 .1 Model description . . . . . . . . . . . . . . . . . . . 44

2.6 .2 Results and discussion . . . . . . . . . . . . . . . . . 47

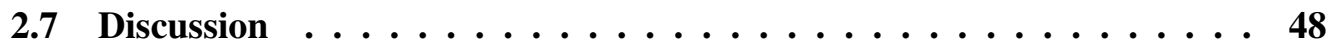

2.7.1 Comparison of four numerical approaches . . . . . . . . . . . 48

2.7.2 Typical impact behaviors of cold sprayed particle and substrate . . . 50

2.8 Summary . . . . . . . . . . . . . . . . . . . 54 


\subsection{Problem description}

This example simulates the normal impact of a deformable Aluminum (Al) spherical particle onto a deformable Aluminum ( $\mathrm{Al}$ ) substrate. Critical velocity of $\mathrm{Al} / \mathrm{Al}$ (particle/substrate) is estimated to be $766 \mathrm{~m} / \mathrm{s}$ [GRU 04] by using shear localization analysis. The experimental value [MAN 11b] from our collaborators is around $780 \mathrm{~m} / \mathrm{s}$. The predicted value of critical velocity by using the Finite Element Analysis (FEA) is slightly higher than the experimental value, therefore, the impact velocities of $700 \mathrm{~m} / \mathrm{s}$ is chosen for analysis in this chapter. The particle size is chosen to correspond to the mean particle size in the cold spray process: $D_{p}=25 \mu \mathrm{m}$. The static dry friction coefficient $\mu_{s}$ of Al/Al is 1.05 [RAM 04]. At low velocities the friction is independent of the relative surface velocity, but at higher velocities the coefficient of friction decreases. For cold spray impact, the particle surface and substrate surface begin to slide relative to each other. The kinetic friction coefficient $\mu_{k}$ should be used instead of $\mu_{s}$ and is generally lower than $\mu_{s}$ for the same material. In this chapter the friction coefficient is set to be 0.3 for all the impacts [YIL 11]. The initial temperature of particle is assumed to be $300 \mathrm{~K}$ for the simple consideration [BAE 08], as well as for the substrate. The effects of velocity, temperature and friction coefficient will be discussed in detail in the next chapter. All the simulations are performed by using the ABAQUS/Explicit version 6.11-2, and the simulation time is fixed at $60 \mathrm{~ns}$ which is enough to observe the whole contact process [LI 09b, LI 09a].

\subsection{Basic assumptions}

The assumptions in creating the numerical model are:

- The shape of Al particle is allowed to be spherical according to the SEM observation (see FIG.2.1);

- Compared with the size of particles, the substrate dimension is a half-space, the cylinder is chosen to represent the substrate in order to keeping the computation as efficient as possible;

- This high pressure region of gas flow near the interface will result in an off-normal impact of particle on the substrate due to the bow shock effect, but in this chapter, we only consider the normal impact without rotation of particle, and the oblique impact will be discussed in chapter 4;

- For the first step of this work, the adhesion model is not implemented in the numerical model. 


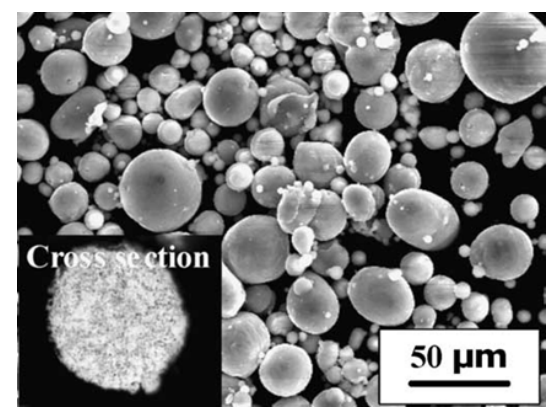

Figure 2.1: SEM image of $\mathrm{Al}$ feedstock powder

\subsection{Lagrangian}

\subsubsection{Model description}

\subsubsection{Geometry}

Because of geometrical and loading symmetries, only half the sphere and half the substrate are considered. FIG.2.2 shows the schematic diagram of 2D axisymmetric model, where the letters $R$ and $H$ refer to the radius and height separately and subscripts $p$ and $s$ stand for particle and substrate. In Finite Element (FE) simulations of the normal impact of a sphere with a half space, on one hand, the substrate should be large enough to eliminate the constrains of boundary conditions; on the other hand, it should not be unnecessarily large, which may increase the computation cost. To achieve this, for the high impact velocity considered in this study, the substrate is modeled as a cylinder having a radius $R_{s}$ and height $H_{s} 10$ times of particle radius $R_{p}$, in order to ensure that the reflecting waves from the substrate bottom and far edges reach the impact zone only after the rebound of particle, as depicted in FIG.2.2. More detailed information about the influence of the substrate size in simulations of the normal impact of a sphere with a half-space could be found in Appendix A.

\subsubsection{Material}

Thermal response is described by specific heat, thermal conductivity. Material properties for thermal response are entered into ABAQUS as temperature dependent, and the data is taken from MPDB software (version 7. 01 demo, 2003, JAHM Software, Inc.) and displayed in the Appendix B completely. List of material properties [BAE 08, YIN 11, MAN 11b] used in simulations is given in TAB.??, at room temperature.

Mie-Grüneisen Equation of State The elastic response of the materials is assumed to follow a linear elasticity model: Mie-Grüneisen equation of state (EOS). In ABAQUS [DAS11, chap.24], the pressure $P$ is determined as a function of the density $\rho$ and the specific energy (internal energy per mass unit) $E_{m}$ : 


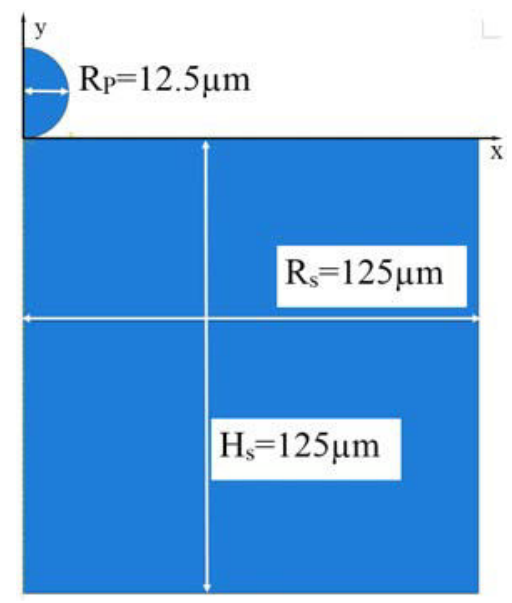

Figure 2.2: Schematic diagram of 2D axisymmetric model.

$$
p-p_{H}=\Gamma \rho\left(E_{m}-E_{H}\right)
$$

Where $P_{H}$ and $E_{H}$ are the Hugoniot pressure and specific energy - functions of the density only, and $\Gamma$ is the Grüneisen ratio defined as:

$$
\Gamma=\Gamma_{0} \frac{\rho_{0}}{\rho}
$$

Where $\Gamma_{0}$ is a material constant and $\rho_{0}$ is the reference density.

The Hugoniot energy, $E_{H}$, is related to the Hugoniot pressure by:

$$
E_{H}=\frac{P_{H} \eta}{2 \rho_{0}}
$$

Where $\eta=1-\rho_{0} / \rho$ is the nominal volumetric compressive strain. Elimination of $\Gamma$ and $E_{H}$ from the above equations yields:

$$
P=P_{H}\left(1-\frac{\Gamma_{0} \eta}{2}\right)+\Gamma_{0} \rho_{0} E_{m}
$$

The equation of state and the energy equation represent coupled equations for pressure and internal energy. ABAQUS/Explicit solves these equations simultaneously at each material point. A common fit to the Hugoniot data is given by:

$$
P_{H}=\frac{\rho_{0} c_{0}^{2} \eta}{(1-s \eta)^{2}}
$$

Where $c_{0}$ and $s$ define the linear relationship between the linear shock velocity, $U_{s}$, and the particle velocity, $U_{p}$, as follows:

$$
U_{s}=c_{0}+s U_{p}
$$


With the above assumptions the linear $U_{s}-U_{p}$ Hugoniot form is written as:

$$
P=\frac{\rho_{0} c_{0}^{2} \eta}{(1-s \eta)^{2}}\left(1-\frac{\Gamma_{0} \eta}{2}\right)+\Gamma_{0} \rho_{0} E_{m}
$$

Where $\rho_{0} c_{0}^{2}$ is equivalent to the elastic bulk modulus at small nominal strains.

Johnson-Cook plasticity model Johnson-Cook hardening is a particular type of isotropic hardening where the static yield stress [ $\mathrm{JOH} 83], \sigma$, is expressed as:

$$
\sigma=\left[A+B \varepsilon^{n}\right]\left[1+C \ln \dot{\varepsilon}^{*}\right]\left[1-T^{* m}\right]
$$

Where $\varepsilon$ is the equivalent plastic strain, $\dot{\varepsilon}^{*}=\dot{\varepsilon} / \dot{\varepsilon}_{0}$ is the dimensionless plastic strain rate for $\dot{\varepsilon}_{0}=1.0 \mathrm{~s}^{-1}$ and $T^{*}=\left(T-T_{0}\right) /\left(T_{m}-T_{0}\right)$ is the homologous temperature. $n$ is the working hardening exponent and $A, B, C$ and $m$ are material constants. $T$ is the temperature in Kelvin, $T_{m}$ is the melting temperature of the material and $T_{0}$ is a reference temperature. The expression in the first set of brackets gives the stress as a function of strain for $\dot{\varepsilon}^{*}=1$ and $T^{*}=0$. The expressions in the second and third sets of brackets represent the effects of strain rate and temperature, respectively. The values for the material constants are also given in TAB.2.1.

We must be very cautious that, the Johnson-Cook plasticity model is more suitable for describing the material behaviors which has the strain rate ranging $10^{3}$ to $10^{5} s^{-1}$; instead, for the material used in the cold spray technology, it may undergo the strain rate up to $10^{7} s^{-1}$. Although more complicated models may indeed give more accurate descriptions of material behavior, and various models may give better descriptions for various materials. The computational codes, in many instances, cannot readily incorporate complicated and diverse models. That is the limitation of this model, thus we would like to obtain the first-hand experimental data of materials in the future in order to perfect our simulation. SHROT [SHR 11] proposed an inverse method of identifying the JohnsonCook parameters from machining experiments. The Levenberg-Marquardt Algorithm was used to re-identify the material parameters by looking at the Chip-morphology and the Cutting force evolution. It seems a good and less expensive way to solve this problem.

\subsubsection{Step}

The analysis of cold spray particle impact onto the substrate accounted for strain hardening, strain-rate hardening, thermal softening, and heating due to frictional, plastic and viscous dissipation. This impact problem includes significant heating due to inelastic deformation of the material which, in turn, changes the material properties. For such case the thermal and mechanical solutions must be obtained simultaneously rather than sequentially. ABAQUS/Expicit provides the Fully coupled thermal-stress analysis and coupled temperature-displacement elements for this purpose. The comparison of different analysis procedures including Dynamic-Explicit including adiabatic heating analysis, Dynamic Temperature-Displacement Explicit analysis, are presented in Appendix C, which give more information about the capacity of each analysis procedure. 


\begin{tabular}{|c|c|}
\hline Properties (Unit) & Aluminum \\
\hline Density, $\rho\left(\mathrm{kg} / \mathrm{m}^{3}\right)$ & $2.7 \times 10^{3}$ \\
Shear modulus, $G(\mathrm{GPa})$ & 27 \\
Thermal conductivity, $\lambda(\mathrm{W} / \mathrm{m} \cdot \mathrm{K})$ & 237.2 \\
Specific heat, $\mathrm{c}(\mathrm{J} / \mathrm{kg} \cdot \mathrm{K})$ & 898.2 \\
Inelastic heat fraction, & 0.9 \\
Sound velocity, $c_{0}(\mathrm{~m} / \mathrm{s})$ & 5386 \\
Slope in $U_{s}$ versus $U_{p}, s$ & 1.339 \\
Grüneisen coefficient, $\Gamma_{0}$ & 1.97 \\
Yield stress, $A(\mathrm{MPa})$ & 148.4 \\
Hardening constant, $B(\mathrm{MPa})$ & 345.5 \\
Hardening exponent, $n$ & 0.183 \\
Strain rate constant, $C$ & 0.001 \\
Thermal softening exponent, $m$ & 0.895 \\
Melting temperature, $T_{m}(K)$ & 916 \\
Reference temperature, $T_{0}(K)$ & 293 \\
\hline
\end{tabular}

Table 2.1: Material properties of Aluminum used in the CEL analysis.

Governing equations The explicit dynamic analysis procedure is based upon the implementation of an explicit integration rule together with the use of diagonal ('lumped') element mass matrices. The equations of motion for the body are integrated using the explicit central-difference integration rule:

$$
\begin{aligned}
& \dot{\mu}_{(i+1)}^{N}=\dot{\mu}_{\left(i-\frac{1}{2}\right)}^{N}+\frac{\Delta t_{i+1}+\Delta t_{i}}{2} \ddot{\mu}_{(i)}^{N}, \\
& \mu_{(i+1)}^{N}=\mu_{(i)}^{N}+\Delta t_{i+1} \dot{\mu}_{\left(i+\frac{1}{2}\right)}^{N}
\end{aligned}
$$

Where $\mu^{N}$ is a degree of freedom (a displacement or rotation component) and the subscript $i$ refers to the increment number in an explicit dynamic step. The central-difference integration operator is explicit in the sense that the kinematic state is advanced using known values of $\dot{\mu}_{\left(i-\frac{1}{2}\right)}^{N}$ and $\ddot{\mu}_{(i)}^{N}$ from the previous increment.

The explicit integration rule is quite simple but by itself does not provide the computational efficiency associated with the explicit dynamic procedure. The key to the computational efficiency of the explicit procedure is the use of diagonal element mass matrices because the accelerations at the beginning of the increment are computed by:

$$
\ddot{\mu}_{(i)}^{N}=\left(M^{N J}\right)^{-1}\left(P_{(i)}^{J}-I_{(i)}^{J}\right)
$$

Where $M^{N J}$ is the mass matrix, $P^{J}$ is the applied load vector, and $I^{J}$ is the internal force vector. A lumped mass matrix is used because its inverse is simple to compute and because the vector multiplication of the mass inverse by the inertial force requires only $n$ 
operations, where $n$ is the number of degrees of freedom in the model. The explicit procedure requires no iterations and no tangent stiffness matrix. The internal force vector, $I^{J}$, is assembled from contributions from the individual elements such that a global stiffness matrix need not be formed.

In ABAQUS/Explicit the heat transfer equations are integrated using the explicit forward-difference time integration rule:

$$
\theta_{(i+1)}^{N}=\theta_{(i)}^{N}+\Delta t_{i+1} \dot{\theta}_{(i)}^{N}
$$

where $\theta^{N}$ is the temperature at node $N$ and the subscript $i$ refers to the increment number in an explicit dynamic step. The forward-difference integration is explicit in the sense that no equations need to be solved when a lumped capacitance matrix is used. The current temperatures are obtained using known values of $\dot{\theta}_{(i)}^{N}$ from the previous increment. The values of $\dot{\theta}_{(i)}^{N}$ are computed at the beginning of the increment by:

$$
\dot{\theta}_{(i)}^{N}=\left(C^{N J}\right)^{-1}\left(P_{(i)}^{J}-F_{(i)}^{J}\right)
$$

where $C^{N J}$ is the lumped capacitance matrix, $P^{J}$ is the applied nodal source vector, and $F^{J}$ is the internal flux vector.

Stability The explicit procedure integrates through time by using many small time increments. The central-difference and forward-difference operators are conditionally stable. The stability limit for both operators (with no damping in the mechanical solution response) is obtained by choosing:

$$
\Delta t \leqslant \min \left(\frac{2}{\omega_{\max }}, \frac{2}{\lambda_{\max }}\right)
$$

where $\omega_{\max }$ is the highest frequency in the system of equations of the mechanical solution response and $\lambda_{\max }$ is the largest eigenvalue in the system of equations of the thermal solution response.

An approximation to the stability limit for the forward-difference operator in the thermal solution response is given by:

$$
\Delta t \approx \frac{L_{\min }^{2}}{2 \alpha}
$$

where $L_{\min }$ is the smallest element dimension in the mesh and $\alpha=\frac{k}{\rho c}$ is the thermal diffusivity of the material. The parameters $k, \rho$, and $c$ represent the material's thermal conductivity, density, and specific heat, respectively.

Two types of estimates are used to determine the stability limit: element-by-element for both the thermal and mechanical solution responses and global for the mechanical solution response. The element-by-element estimate is determined using the smallest time increment size due to the thermal and mechanical solution responses in each element, and 
selected as the estimate in this study. The element-by-element estimate is conservative; it will give a smaller stable time increment than the true stability limit, which is based upon the maximum frequency of the entire model. In general, constraints such as boundary conditions and kinematic contact have the effect of compressing the eigenvalue spectrum, and the element-by-element estimates do not take this into account.

Time incrementation The time increment used in an analysis must be smaller than the stability limits of the central- and forward-difference operators. Failure to use such a time increment will result in an unstable solution. When the solution becomes unstable, the time history response of solution variables, such as displacements, will usually oscillate with increasing amplitudes. The total energy balance will also change significantly.

ABAQUS/Explicit has two strategies for time incrementation control: fully automatic time incrementation (where the code accounts for changes in the stability limit) and fixed time incrementation. The default time incrementation scheme in ABAQUS/Explicit is fully automatic and it is used in this study.

\subsubsection{Interaction}

ABAQUS/Explicit provides two algorithms for modeling contact interactions. The General contact algorithm allows very simple definitions of contact with very few restrictions on the types of surfaces involved. The Contact pair algorithm has more restrictions on the types of surfaces involved and often requires more careful definition of contact; however, it allows some interaction behaviors that currently are not available with the general contact algorithm. For 2D surfaces, the contact pair algorithm must be used although the general contact algorithm is more powerful and allows for simpler contact definitions. More information about the Contact pair algorithm are displayed in Appendix D.

The definition of a contact pair interaction in ABAQUS/Explicit consists of specifying:

- The surfaces that interact with one another;

- The contact property models;

- The contact formulation;

- The contact constraint enforcement method.

The two surfaces involved in the surface-to-surface contact are pointed out in FIG.2.3, $A S U R F$ and BSURF. BSURF represents the outer spherical surface of particle, meanwhile $A S U R F$ represents a circular flat surface of substrate with radius of $4 \mathrm{R}=50 \mu \mathrm{m}$.

The contact pressure-overclosure relationship used in this study is referred to as the 'Hard' contact contact model. The classical isotropic Coulomb friction model is used for modeling the friction relationship between particle and substrate.

For two element-based deformable surfaces contact each other, the balanced masterslave weighting contact algorithm is used. Due to the nonlinear geometric effect of particle, one chooses the Finite sliding formulation, which is the more general and allows any arbitrary motion of the surfaces. 


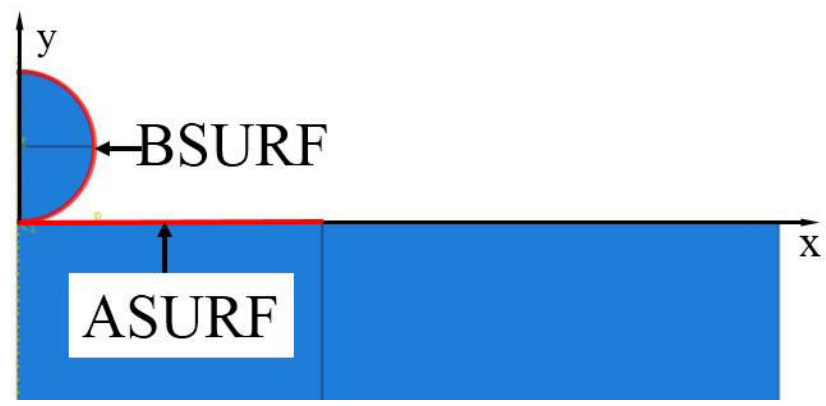

Figure 2.3: Schematic diagram of surface-to-surface contact.

Contact pairs in ABAQUS/Explicit use kinematic enforcement by default, but penalty enforcement is specified for this study. A small penetration between particle and substrate is therefore allowed.

\subsubsection{Boundary conditions}

The following boundary condition 'types' are available in ABAQUS/Explicit:

- XSYMM Symmetry about a plane $X=$ constant $(U 1=U R 2=U R 3=0)$;

- YSYMM Symmetry about a plane $Y=$ constant $(U 2=U R 1=U R 3=0)$;

- ZSYMM Symmetry about a plane $Z=$ constant $(U 3=U R 1=U R 2=0)$;

- ENCASTRE Fully built-in $(U 1=U 2=U 3=0)$;

- PINNED Pinned $(U 1=U 2=U 3=U R 1=U R 2=U R 3=0)$.

The boundary conditions used in this study are shown in FIG.2.4.

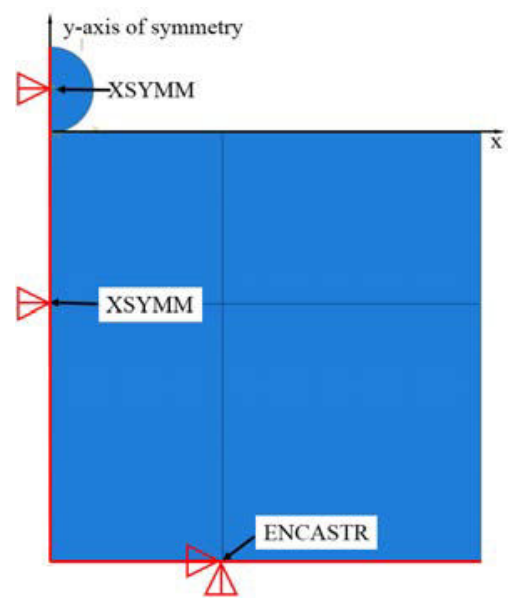

Figure 2.4: Schematic diagram of boundary condition of 2D axisymmetry model. 


\subsubsection{Initial conditions}

The value of impact velocity and temperature are specified in the predefined field during the analysis, as illustrated in FIG.2.5 .

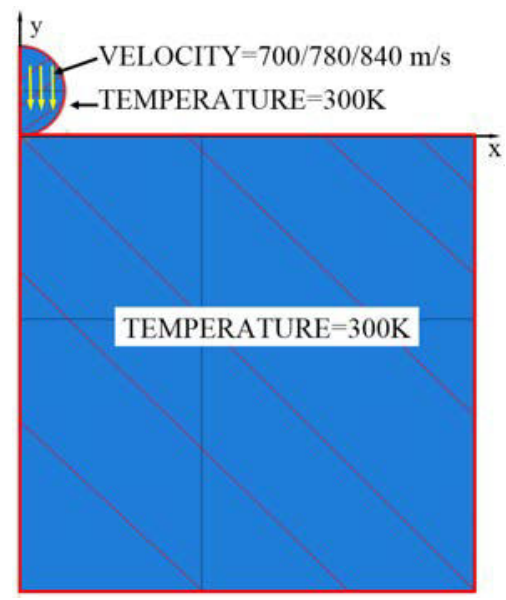

Figure 2.5: Schematic diagram of initial conditions of velocity and temperature.

\subsubsection{Mesh}

Element type The element type CAX4RT (a 4-node thermally coupled axisymmetric quadrilateral, bilinear displacement and temperature, reduced integration, viscoelastic hourglass control ) is used in this study.

In first-order axisymmetric quadrilateral element, the strain operator provides constant volumetric strain throughout the element. This constant strain prevents mesh 'locking' when the material response is approximately incompressible. Second-order elements are excluded in this study because they provide higher accuracy in ABAQUS/Standard than first-order elements for 'smooth' problems without involving complex contact conditions, impact, or severe element distortions. Only reduced-integration first-order elements are available for quadrilateral elements in ABAQUS/Explicit; the elements with reduced integration are also referred to as uniform strain or centroid strain elements with hourglass control. Reduced integration reduces running time, especially in three dimensions.

Mesh control The structured meshing technique is applied to whole region that has been assigned the Quad element shape option. The mesh algorithm of minimize the mesh transition is used in order to reduce mesh distortion when the mesh moves from a coarse mesh to a fine mesh.

Mesh size In this study, the mesh size of $0.5 \mu m\left(1 / 50 D_{p}\right)$ is used in the particle and 4R $\times 4 \mathrm{R}$ substrate region for obtaining more accurate simulation results. The mesh structure could be seen in FIG.2.6 in details. 


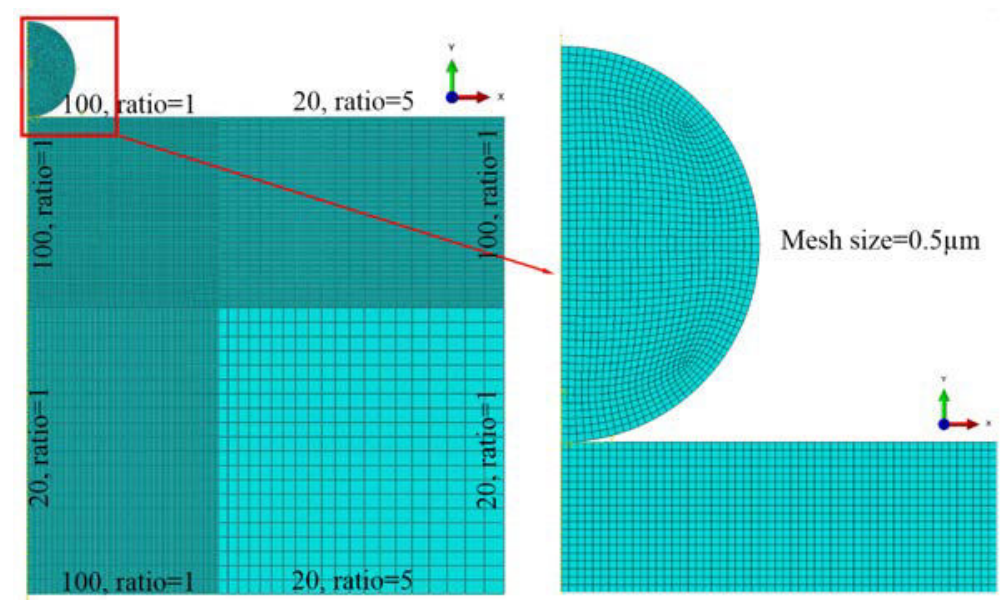

Figure 2.6: Mesh structure of the 2D axisymmetry model.

\subsubsection{Results and discussion}

FIG.2.7a shows the distribution of equivalent plastic strain for Al particle and $\mathrm{Al}$ substrate at impact velocity of $700 \mathrm{~m} / \mathrm{s}$. The calculation could not finish completely, and stops at $19 \mathrm{~ns}$. It can be observed that the maximum plastic strain is located at the interface between particle and substrate. The Lagrangian approach predicts that at this given impact velocity of $700 \mathrm{~m} / \mathrm{s}$, the spherical particle is flatten and a crater is generated in the flat substrate. Intensive deformation is observed at the outer region of the interface between the $\mathrm{Al}$ particle and the $\mathrm{Al}$ substrate indicated by the grey color, where the equivalent plastic strain exceeds the value of 2.5 .

FIG.2.7b shows the distribution of temperature for $\mathrm{Al}$ particle and $\mathrm{Al}$ substrate with $700 \mathrm{~m} / \mathrm{s}$ impact velocity after $19 \mathrm{~ns}$ simulation time. The cold spray process can be seen as adiabatic [ASS 03]. As the temperature of material is related to the plastic deformation, and since the particle undergoes large amount of deformation with a maximum strain close to the surface, it is obvious that the maximum temperature should be found nearby the interface.
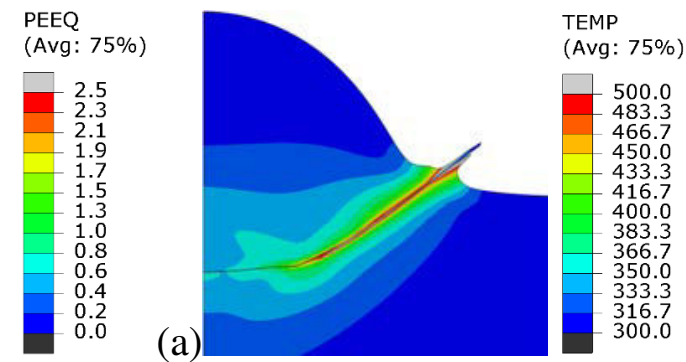

(b)

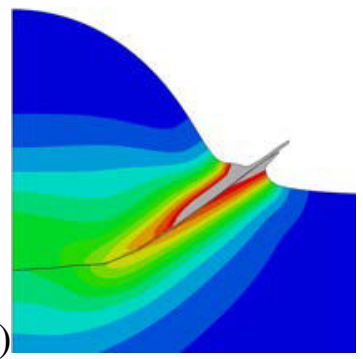

Figure 2.7: Contours of (a) equivalent plastic strain and (b) temperature of $\mathrm{Al} / \mathrm{Al}$ by using the Lagrangian numerical approach at $700 \mathrm{~m} / \mathrm{s}$ - aborted at $19 \mathrm{~ns}$.

The outer region of the interface between the Al particle and the Al substrate deformed 
into a small and thin jet-region since $5 \mathrm{~ns}$. This material jet with linear edges becomes much sharper and longer along with the contact time increasing as shown in FIG.2.8. Take the element 515 of particle as a example, which has the maximum X-direction displacement, it is prolonged more than 15 times along the X-direction. Both the particle and the substrate participate in the forming of the jet. It leads to convergence difficulties, resulting in the calculations fail permanently. The excessive mesh distortion is of significant concern in this result.

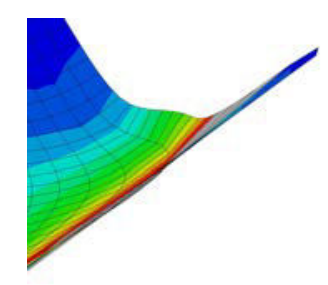

(a) (b)

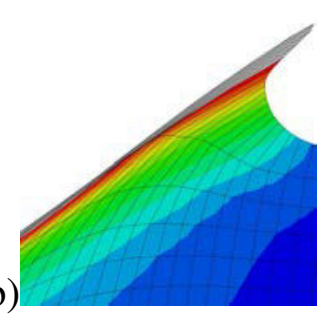

Figure 2.8: Element distortion of (a) Al particle and (b) Al substrate by using the Lagrangian numerical approach at $700 \mathrm{~m} / \mathrm{s}$.

In the subsequent analysis, there is a so-called key element at the lower surface of the particle or the upper surface of the substrate which experiences the highest value of equivalent plastic strain or temperature. The evolution of the equivalent plastic strain for the key element - located at the particle/substrate interface - is shown in FIG.2.9a. The results show that at the given impact velocity $(700 \mathrm{~m} / \mathrm{s})$, the equivalent plastic strain increases changes monotonically during the contact time, eventually reaching a plateau. The contact at the beginning is drastic, the strain rate is quite high between $2 n s$ and $5 n s$, reaching $10^{6} s^{-1}$. After that, the strain rate decreases, but still stays at the level of $10^{5} s^{-1}$. The particle side undergoes larger plastic strain than that of substrate side, thus obtains higher temperature as the FIG.2.9b shows. A sharp temperature rise of particle is observed after a dwell time ranging between $5 \mathrm{~ns}$ and $15 \mathrm{~ns}$, followed by the abortion of calculation. Comparing the FIG.2.9a and FIG.2.9b, it is clear to conclude that the distortion of particle element $(\mathrm{PEEQ}=0)$ leads to the sudden unreasonable change of temperature.

\subsection{Arbitrary Lagrangian Eulerian}

\subsubsection{Model description}

All the model description including geometry, material, analysis procedure, interaction, boundary and initial conditions, element type, mesh structure and outputs are exactly the same as model descriptions described in section 2.3.1, one could see section 2.3.1 for details. The subsequent subsections describe the various options that can be used with adaptive meshing. More detailed information about the ALE adaptive mesh controls are displayed in Appendix E. 
(a)

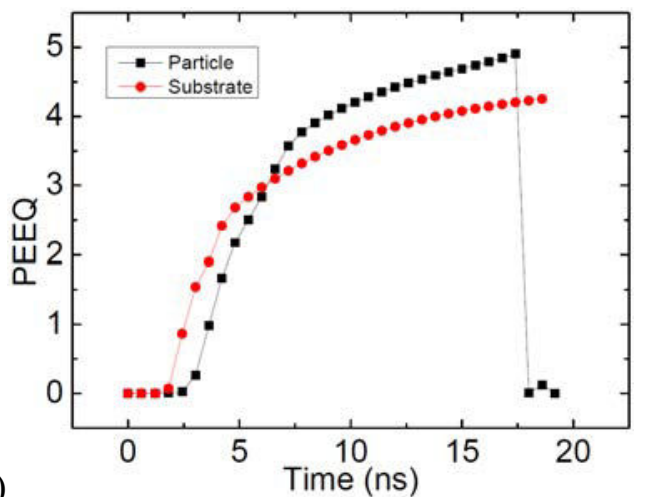

(b)

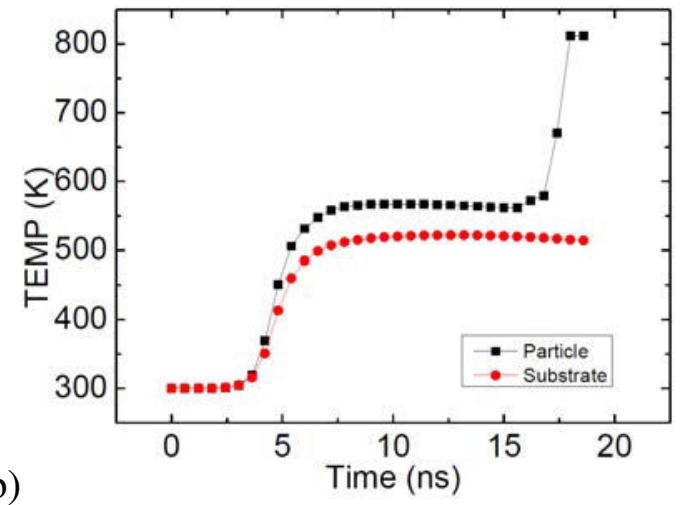

Figure 2.9: Time histories of (a) equivalent plastic strain (PEEQ) and (b) temperature of $\mathrm{Al} / \mathrm{Al}$ by using the Lagrangian numerical approach at $700 \mathrm{~m} / \mathrm{s}$.

\subsubsection{Adaptive meshing domain}

Adaptive meshing can be applied to an entire model or to individual parts of a model. Lagrangian adaptive mesh domains are usually used to analyses transient problems with large deformations. A Lagrangian adaptive mesh domain is created as FIG.2.10 shows, where surrounded by the red lines, so that the domain as a whole will follow the material originally inside it, which is the proper physical interpretation for most structural analyses. Defining adaptive mesh domains that cover only a fraction of the entire problem domain will reduce the cost proportionally. On the boundary of a Lagrangian domain the mesh will follow the material in the direction normal to the boundary, so that the mesh covers the same material domain at all times.

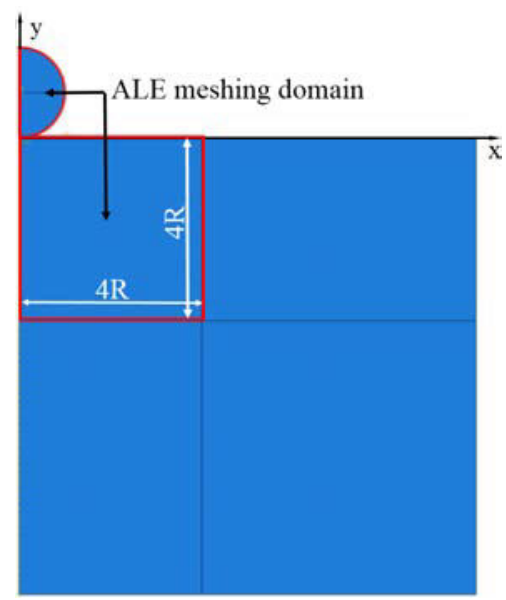

Figure 2.10: Schematic diagram of the ALE adaptive mesh domain. 


\subsubsection{Frequency and intensity}

In most cases the frequency of adaptive meshing is the parameter that most affects the mesh quality and the computational efficiency of adaptive meshing. A typical adaptive mesh application with Lagrangian boundaries will require adaptive meshing every 5 to 100 increments.

In an adaptive meshing increment, a new, smoother mesh is created by sweeping iteratively over the adaptive mesh domain. During each mesh sweep, nodes in the domain are relocated - based on the current positions of neighboring nodes and elements - to reduce element distortion. In a typical sweep a node is moved a fraction of the characteristic length of any element surrounding the node. Increasing the number of sweeps increases the intensity of adaptive meshing in each adaptive meshing increment.

The default frequency for adaptive meshing is 10 , and the default number of mesh sweeps is 1 . The default values are usually adequate for low to moderate rate dynamic problems and for quasi-static process simulations undergoing moderate deformation. If the frequency or number of mesh sweeps is too low, excess element distortion may cause the analysis to terminate before the mesh is adapted; or, if a solution can be obtained, it may not be as accurate as the solution that could be obtained with a higher quality mesh. For high - rate impact problems undergoing large amounts of deformation, it may be necessary to increase the frequency of adaptive meshing or the number of mesh sweeps.

\subsubsection{Meshing smoothing}

For adaptive mesh domains with Lagrangian boundary regions, the objective of the mesh smoothing methods is to minimize mesh distortion while improving element aspect ratios, at the expense of diffusing initial mesh gradation. The uniform mesh smoothing objective is recommended for problems with moderate to large overall deformation.

The conventional forms of the basic smoothing methods do not perform well in highly distorted domains. To ensure the robustness of adaptive meshing, ABAQUS/Explicit uses geometrically enhanced forms of the basic smoothing algorithms (Enhanced algorithm based on evolving element geometry).

If an adaptive mesh domain has no Eulerian boundary regions, then the mesh sweeps are based on current nodal locations (Current deformed position), which account for material motion accumulated since the last adaptive mesh increment. This approach is generally the best for Lagrangian problems that undergo large overall deformation.

\subsubsection{Boundary region smoothing}

Geometric features are identified initially as edges on boundary regions where the angle between the normals on adjacent element faces is greater than the initial geometric feature angle, $\theta_{I}\left(0^{\circ} \leq \theta_{I} \leq 180^{\circ}\right)$, see FIG.2.11.

During each mesh sweep in an adaptive mesh increment, nodes along a geometric edge are positioned by applying the basic smoothing methods. The nodes are constrained to lie along the discrete geometric edge unless the angle forming the geometric edge 

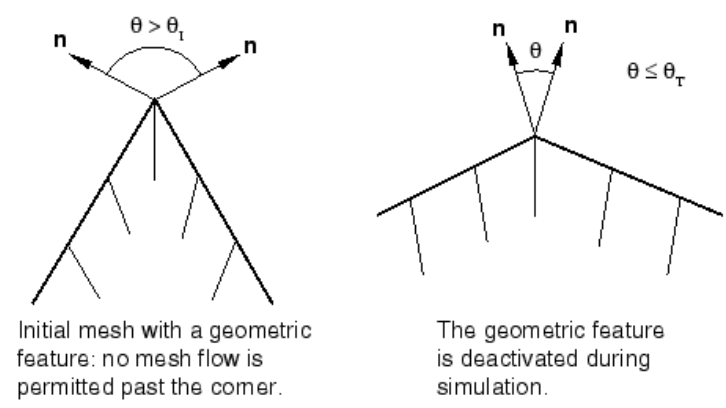

Figure 2.11: Schematic diagrams of detection and deactivation of geometric features.

becomes less than the transition feature angle, $\theta_{T}\left(0^{\circ} \leq \theta_{T} \leq 180^{\circ}\right)$. If the angle across the geometric edge becomes less than $\theta_{T}=30^{\circ}$, the boundary surface is considered to be flattened sufficiently for the feature to be deactivated, and the mesh is allowed to slide freely over the material (unconstrained by the deactivated geometric edge). Geometric corners are allowed to flatten in a similar fashion. This logic allows great flexibility in mesh adaptation while preserving geometric features in the model.

\subsubsection{Advection}

Element and material state variables must be transferred from the old mesh to the new mesh in each advection sweep. The number of variables to be advected depends on the material model and element formulation; however, stress, history variables, density, and internal energy are always solution variables.

Second-order advection is used for all adaptive mesh domains. Nodal velocities are computed on a new mesh by first advecting momentum, then using the mass distribution on the new mesh to calculate the velocity field. Advecting momentum directly ensures that momentum is conserved properly in the adaptive mesh domain during remapping.

The half-index shift method is computationally more intensive, but it may result in less wave dispersion for some problems. This method first shifts each of the nodal momentum variables from the nodes surrounding an element to the element center. The shifted momentum variables are then advected from the old mesh to the new mesh by the same second-order algorithms used for advecting element variables, providing momentum variables at the center of the new elements. Finally, the momentum variables at the element centers in the new mesh are shifted back to the nodes.

\subsubsection{Results and discussion}

The same single normal impact problem is solved by using the ALE approach, FIG.2.12 shows the distribution of equivalent plastic strain and temperature of $\mathrm{Al} / \mathrm{Al}$ at $700 \mathrm{~m} / \mathrm{s}$ after $60 \mathrm{~ns}$ simulation time. There is no excessive mesh distortion by using the ALE numerical approach. The material jet at the outer region of interface appears smoother instead of thin and acute, noting that the default values of Frequency 10 and 
Remeshing sweeps per increment 1 are applied to the calculation. The gap between particle and substrate is due to the lack of adhesion model. The particle rebounds after the contact time of $30 \mathrm{~ns}$. It is shown that the outer edge side of the particle/substrate interface experiences a higher temperature than the center of the impact due to the localization of the plastic deformation within a region close to the interface.
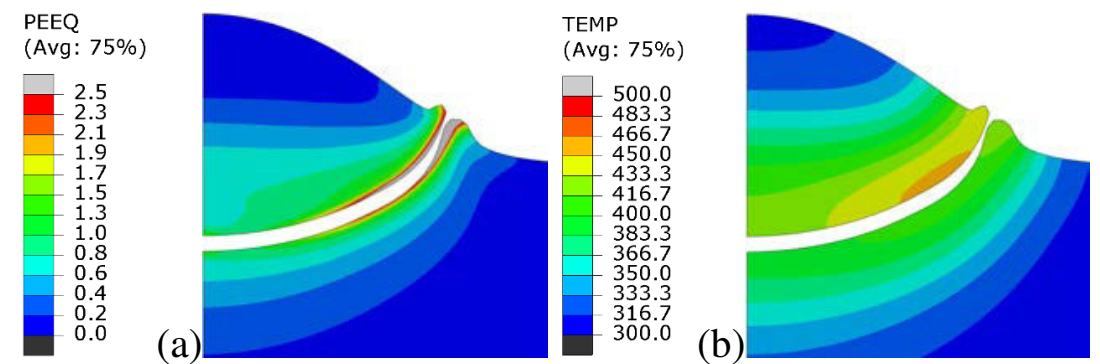

Figure 2.12: Contours of (a) equivalent plastic strain and (b) temperature of $\mathrm{Al} / \mathrm{Al}$ by using the ALE numerical approach at $700 \mathrm{~m} / \mathrm{s}$.

The evolution of the equivalent plastic strain for the key element is shown in FIG.2.13a for both the particle side and substrate side. It shows a slowly decreasing of the equivalent plastic strain over time after reaching the peak value quickly. In fact, however, the history of equivalent plastic strain should be another way in a pure Lagrangian analysis, which means the equivalent plastic strain increases monotonically, eventually reaching a plateau. This unrealistic behavior is attributed to interpolation errors caused by the adaptive meshing algorithm in the remapping phase and to the presence of high straingradients in the vicinity of the interface [YIL 11]. When an adaptive meshing is used with some similarities with the CEL approach, integration points and material points start to deviate. Therefore the motion of the mesh on the interior of an adaptive mesh domain represents the composite effects of the material motion and adaptive meshing. Since element integration point variables do not necessarily represent values at the same material point throughout the step, the output at elements and nodes must be different from that in a pure Lagrangian analysis.

The temperature at the point where the maximum is found is plotted versus time in FIG.2.13b. A drastic temperature rise is observed after a dwell time ranging between $5 \mathrm{~ns}$ and $10 n s$, followed by a gradual drop along with the simulation time prolonged. The temperature of particle is higher than that of substrate due to the larger plastic deformation of particle. It should be mentioned that the melting temperature is never reached. Note that the friction energy plays a minor part in the internal energy, which reaches the maximum value of $8 \%$ at the moment of $2.5 \mathrm{~ns}$ and keeps below $0.62 \%$ during the whole process.

The simulation results and computational cost are influenced by the frequency and intensity. The choice of parameter value affects the analysis processes whether success or fail. For example, if the frequency equals to 15 , and the intensity remains 1 , the calculation is aborted unexpectedly. Moreover, the simulation results obtained from the ALE numerical approach are not unique, the different choice leads to different deformation ratios and predicted value of maximum equivalent plastic strain/temperature of particle 
(a)

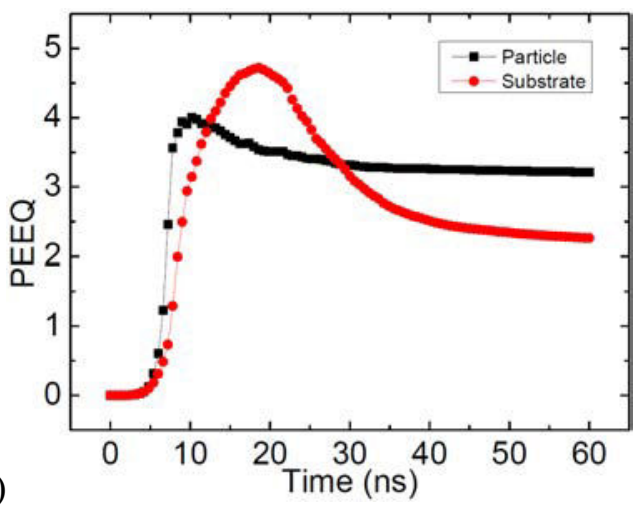

(b)

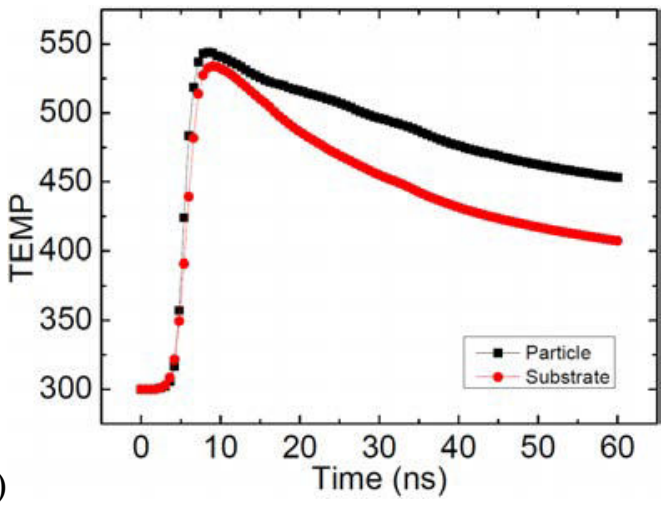

Figure 2.13: Time histories of (a) equivalent plastic strain (PEEQ) and (b) temperature of Al/Al by using the ALE numerical approach at $700 \mathrm{~m} / \mathrm{s}$.

as the ТАВ.2.2 illustrates. Compressed height of particle divided by its deformed diameter is the deformation ratio. It is generally less expensive to increase the number of mesh sweeps slightly before increasing the frequency, as long as the number of advection sweeps remains small. Increasing the number of mesh sweeps from 1 to 5 (case 1 , case 2 ), the computational time even reduces $8.3 \%$ at impact velocity of $700 \mathrm{~m} / \mathrm{s}$; however, increasing the frequency from 10 to 15 (case 2, case 3 ), the computation cost increases $1 \%$.

\begin{tabular}{|c|c|c|c|}
\hline Case & Frequency & Intensity & Deformation ratio \\
\hline 1 & 10 & 1 & $46.48 \%$ \\
2 & 10 & 5 & $47.09 \%$ \\
3 & 15 & 5 & $46.42 \%$ \\
\hline Case & PEEQ $_{\text {pmax }}$ & TEMP $_{\text {pmax }}(K)$ & Relative CPU Time \\
\hline 1 & 4.669 & 543.6 & 1.000 \\
2 & 5.444 & 573.7 & 0.917 \\
3 & 5.843 & 556.9 & 0.926 \\
\hline
\end{tabular}

Table 2.2: The influences of frequency and intensity on the simulation results at $700 \mathrm{~m} / \mathrm{s}$.

\subsection{Smoothed Particle Hydrodynamics}

\subsubsection{Model description}

Smoothed particle hydrodynamics ( $\mathrm{SPH})$ is a numerical method that is part of the larger family of meshless (or mesh-free) methods. For these methods you do not define nodes and elements as you would normally define in a finite element analysis; instead, only a collection of points are necessary to represent a given body. In smoothed particle hydrodynamics these nodes are commonly referred to as particles or pseudo-particles 
[MAN 11c, CAL 11, MAU 08b]. Note that these particles are different from the powder particles used to produce the Cold Spray coating.

Smoothed particle hydrodynamics is a fully Lagrangian modeling scheme permitting the discretization of a prescribed set of continuum equations by interpolating the properties directly at a discrete set of points distributed over the solution domain without the need to define a spatial mesh. All conservation equations, including conservation of mass, conservation of momentum and conservation of energy are defined with Lagrangian form. The material changes over time due to the interaction between the current pseudo-particle and the neighboring pseudo-particles, and at each time step, the field approximation is done based on the local distribution of neighboring particles. At its core, the method is not based on discrete particles (spheres) colliding with each other in compression or exhibiting cohesive-like behavior in tension as the word particle might suggest. Rather, it is simply a clever discretization method of continuum partial differential equations [CAL 13, MAU 08a, COM 08].

The Al properties are exactly the same as the material of $\mathrm{Al}$ described in section 2.3.1.2, one could see section 2.3.1.2 for details.

\subsubsection{Geometry}

The smoothed particle hydrodynamic approach is not implemented for twodimensional elements. FIG.2.14 shows the pertinent dimensions of 3D model for the impact particle and substrate. Only the particle part is modeled in the SPH reference frame due to its tremendous deformation comparing to the substrate, whereas the substrate is still in the Lagrangian reference frame.

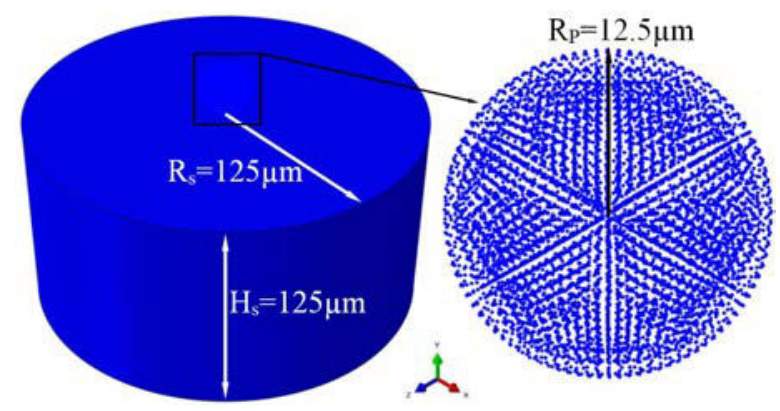

Figure 2.14: Schematic diagram of 3D SPH model.

\subsubsection{Step}

The PC3D element used for the SPH analysis does not support the coupled mechanical - thermal analysis, thus the Explicit dynamic stress/displacement procedure is performed. The explicit central-difference operator satisfies the dynamic equilibrium equations at the beginning of the increment, $t$; the accelerations calculated at time $t$ are used to advance 
the velocity solution to time $t+\Delta t / 2$ and the displacement solution to time $t+\Delta t$. One could see the equations 2.9 and 2.10 for details.

\subsubsection{Interaction}

The contact interaction of particle and substrate is the same as any contact interaction between a node-based surface (associated with the PC3D elements) and an element-based surface. Both general contact and contact pairs can be used. All interaction types and formulations available for contact involving a node-based surface are allowed, including cohesive behavior. The default contact thickness for SPH pseudo-particle is the same value specified as the characteristic length on the section 2.5.1.6 definition; thus, for contact purposes, SPH pseudo-particles behave as spheres with radii equal to the characteristic length, and this concept will be explained in section 2.5.1.6 below.

\subsubsection{Boundary conditions}

The bottom surface of substrate is constrained by the PINNED boundary condition $(U 1=U 2=U 3=U R 1=U R 2=U R 3=0)$.

\subsubsection{Initial conditions}

The impact velocity of $700 \mathrm{~m} / \mathrm{s}$ is applied to the particle part in the predefined field module.

\subsubsection{Mesh}

The substrate uses the C3D8R element type with distortion control and enhanced hourglass control as discussed before, while the particle uses the unique available PC3D element type in ABAQUS/Explicit for the SPH analysis.

Element type The smoothed particle hydrodynamic approach is implemented via the formulation associated with PC3D elements. These 1-node elements are simply a means of defining pseudo - particles in space that model a particular body or bodies, as FIG.2.14 illustrates. These 1-node elements utilize existing functionality in ABAQUS to reference element-related features such as materials, initial conditions, distributed loads, and visualization. There are no hourglass or distortion control forces associated with PC3D elements. These elements do not have faces or edges associated with them.

One could define these elements in a similar fashion as one would define point masses. The coordinates of these points lie either on the surface or in the interior of the body being modeled, similar to the nodes of a body meshed with brick elements. For more accurate results, one should strive to space the nodal coordinates of these PC3D elements as uniformly as possible in all directions.

An alternative to directly defining PC3D elements, which also the method used in this study, is to define conventional continuum finite element types C3D8R and automatically 
convert them to PC3D elements at the beginning of the analysis using solidtosph.py, as presented in Appendix F. A schematic diagram of this transformation process is shown in FIG.2.15.

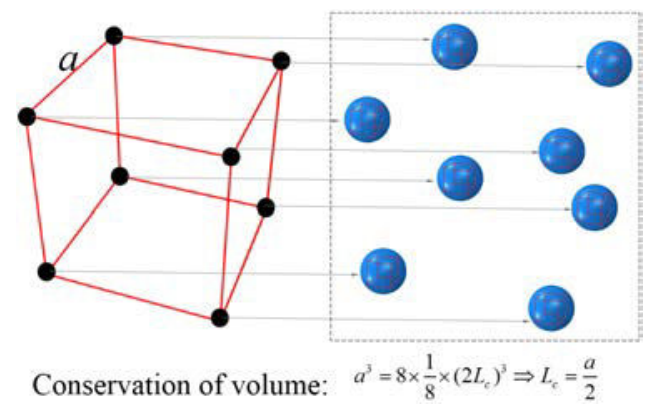

Figure 2.15: Schematic diagram of transformation of C3D8R element to PC3D elements.

SPH kernel function The smoothed particle hydrodynamic approach implemented in ABAQUS/Explicit uses a cubic spline as the interpolation polynomial in the following form:

$$
W^{3 D}(v, h)=\frac{1}{\pi h^{3}} \begin{cases}\left(1-\frac{3}{2} v^{2}+\frac{3}{4} v^{3}\right), & 0 \leq v \leq 1 \\ \frac{1}{4}(2-v)^{3}, & 1 \leq v \leq 2 \\ 0 . & \text { otherwise }\end{cases}
$$

Where $h$ is the smoothing length determining the number of closest neighbors included in the summations and $v=\left|\mathbf{r}_{i}-\mathbf{r}_{j}\right| / h$, as the FIG.2.16 illustrates. It is based on the classical smoothed particle hydrodynamic theory as outlined in the reference [SWE 94].

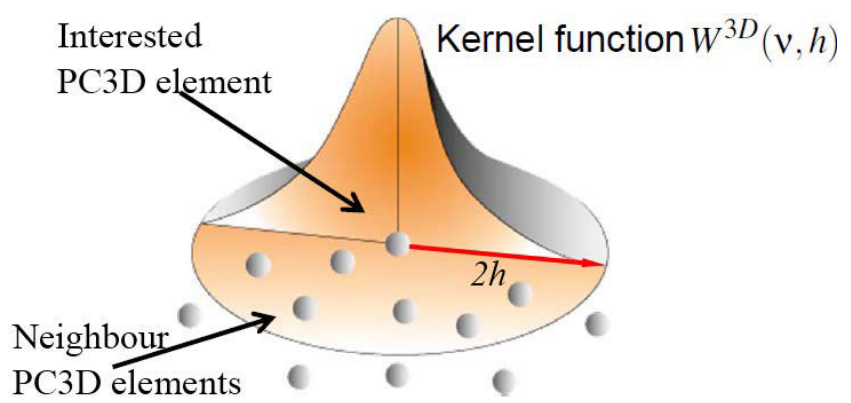

Figure 2.16: Schematic diagram of kernel interpolate function.

Characteristic length There is currently no capability to automatically compute the volume associated with these elements. Hence, one need to supply a characteristic length $L_{c}$ that will be used to compute the element volume, which in turn is used to compute 
the mass associated with the PC3D element. It is assumed that the nodes are distributed uniformly in space and that each node element is associated with a small cube centered at the element (see FIG.2.17). When stacked together, these cubes will fill the overall volume of the body with some minor approximation at the free surface of the body. The characteristic length is half the length of the cube side .

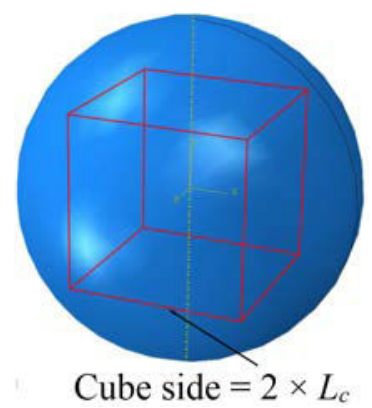

Figure 2.17: Schematic diagram of cube centred at the PC3D element.

From a practical perspective, one could use the half-distance between two nodes of C3D8R element as the characteristic length, according to the transformation pattern of C3D8R elements to PC3D elements (see FIG.2.15), which is $0.625 \mu m$ in this study. When the finite element model is created at the first step, the C3D8R elements are distributed uniformly at the space in order to get accurate simulation results. If the mesh size of 0.5 $\mu m$ is chosen as discussed before, then there will be 1250000 elements in the particle part. It increases the computation cost dramatically. For the time consuming reason, the mesh size has to be $1.25 \mu \mathrm{m}$ in this study. Alternatively, if one knows the mass and density of the part, you can compute the volume of the part and divide it by the total number of particles in the part to obtain the volume of the small cube associated with each particle. Half of the cubic root of this small volume is a reasonable characteristic length for this particle set.

Smoothing length calculation Even though particle elements are defined in the model using one node per element, the smoothed particle hydrodynamic approach computes contributions for each element based on neighboring elements that are within a sphere of influence. The radius of this sphere of influence is referred to in the literature as the smoothing length. The smoothing length is independent of the characteristic length discussed above and governs the interpolation properties of the method. By default, the smoothing length is computed automatically in ABAQUS/Explicit. As the deformation progresses, elements move with respect to each other and, hence, the neighbors of a interested element can (and typically do) change. Every increment ABAQUS/Explicit recomputes this local connectivity internally and computes kinematic quantities (such as normal and shear strains, deformation gradients, etc.) based on contributions from this cloud of particles centered at the particle of interest. Stresses are then computed in a similar fashion as for reduced-integration brick elements, which are in turn used to compute element 
nodal forces for the particles in the cloud based on the smoothed particle hydrodynamic formulation.

By default, ABAQUS/Explicit computes a smoothing length at the beginning of the analysis such that the average number of particles associated with an element is roughly between 30 and 50. The smoothing length is kept constant during the analysis. Therefore, the average number of particles per element can either decrease or increase during the analysis depending on whether the average behavior in the model is expansive or compressive, respectively. If the analysis is mostly compressive in nature, the total number of particles associated with a given element might exceed the maximum allowed and the analysis will be stopped. By default, the maximum number of allowed particles associated with one element is 140 .

Smoothed particle hydrodynamic domain A rectangular region is computed at the beginning of the analysis as the bounding box within which the particles will be tracked. This fixed rectangular box is $10 \%$ larger than the overall dimensions of the whole model, and it is centered at the geometric center of the model. As the analysis progresses, if a PC3D element is outside this box, it behaves like a free-flying point mass and does not contribute to smoothed particle hydrodynamic calculations. If the particle reenters the box at a later stage, it is once again included in the calculations.

\subsubsection{Results and discussion}

The deformation pattern predicted by using SPH approach is dissimilar to the deformation pattern discussed in the above two sections. The particle does not have the material jet around the contact zone, and it is embedded deeper in the substrate as shown in FIG.2.18. Intensive plastic deformation is observed at the region surrounding the contact center of $\mathrm{Al}$ substrate indicated by the grey color, where the equivalent plastic strain exceeds the value of 2.5 .

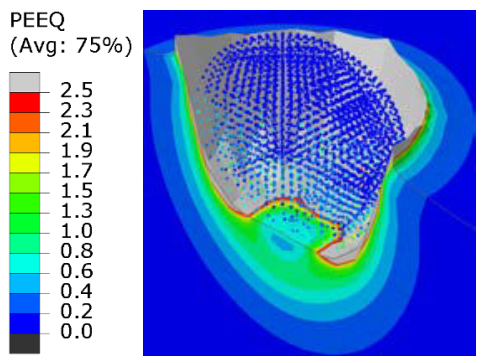

Figure 2.18: Contour of equivalent plastic strain of $\mathrm{Al} / \mathrm{Al}$ by using the $\mathrm{SPH}$ numerical approach at $700 \mathrm{~m} / \mathrm{s}$.

The evolution of the maximum plastic strain along $60 \mathrm{~ns}$ is shown in FIG.2.19a. It can be observed that, the strain of particle tends to an horizontal asymptote, whereas the maximum plastic strain of substrate continues to increase after $30 \mathrm{~ns}$, and this is not reasonable. Under high impact velocity and thus high strain rate, the excessive deformation 
would create a viscous-type resistance which hinder further deformation. This effect is related to the second term of the Johnson-Cook strain-stress law which accounts for strain rate hardening. However, the evolution of the stress is in a different way as FIG.2.19b shows. If considering the thermal softening, the resistance of material to shear flow is general low when the temperature of substrate increases. This means that by approaching the melting temperature, the material loses its shear strength and undergoes excessive deformation for any amount of impose shear stress. However, this is a isotherm analysis, and the temperature of whole system keeps the constant value of $300 \mathrm{~K}$. Moreover, no damage material model is added in the material. This abnormal behavior of substrate surface material may due to the numerical limitations and errors. Much higher equivalent plastic strain is clearly observed using the Dynamic-Explicit procedure. With the Dynamic-Temp-Disp-Explicit procedure, obvious heat conduction from the interface to inner particle and substrate is conducted. Although the Dynamic-Explicit procedure is computationally efficient for the analysis of large models with relatively short response times and for the analysis of extremely discontinuous events, fully coupled thermal-stress analysis is needed when the stress analysis is dependent on the temperature distribution and the temperature distribution depends on the stress solution. For this case, the thermal and mechanical solutions must be obtained simultaneously rather than sequentially.

(a)

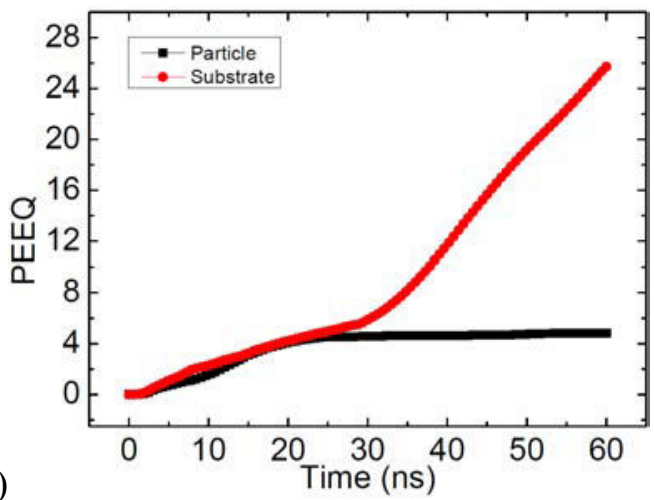

(b)

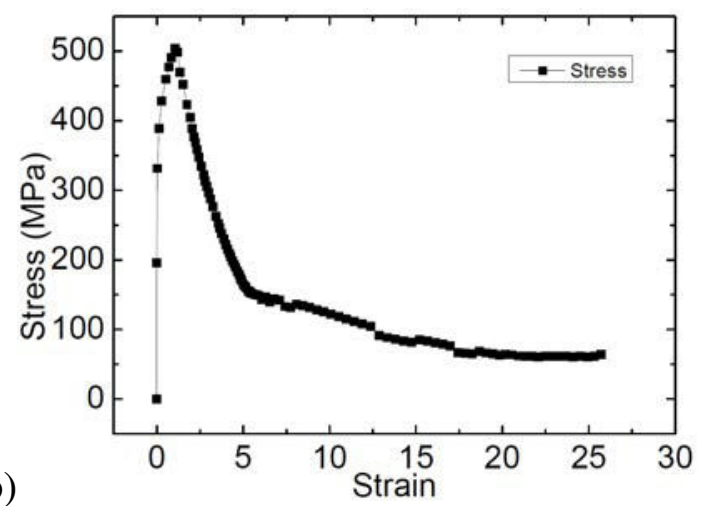

Figure 2.19: Time histories of (a) equivalent plastic strain (PEEQ) of $\mathrm{Al} / \mathrm{Al}$ and (b) stress of substrate by using the SPH approach at $700 \mathrm{~m} / \mathrm{s}$.

\subsection{Coupled Eulerian Lagrangian}

\subsubsection{Model description}

For CEL numerical approach, one also performs the fully coupled thermal-stress analysis and coupled temperature-displacement elements EC3D8RT (Eulerian domain) and C3D8RT (Lagrangian domain) are used for this purpose. 


\subsubsection{Geometry}

The Eulerian approach is implemented only for three-dimensional elements, considering the axial symmetry, the one-fourth model is created. FIG.2.20 shows the pertinent dimensions for the Eulerian domain and Lagrangian domain.

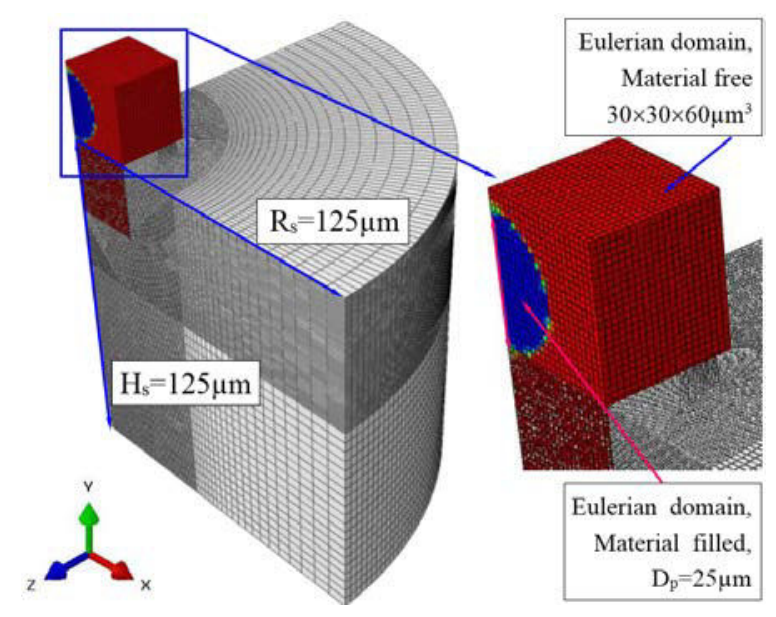

Figure 2.20: Schematic diagram of 3D CEL model.

\subsubsection{Eulerian volume fraction}

The Eulerian implementation in ABAQUS/Explicit is based on the volume-of-fluid method. In this method, material is tracked as it flows through the mesh by computing its Eulerian Volume Fraction (EVF) within each element. By definition, if a material completely fills an element, its volume fraction is one; if no material is present in an element, its volume fraction is zero. Eulerian elements may simultaneously contain more than one material. If the sum of all material volume fractions in an element is less than one, the remainder of the element is automatically filled with 'void' material. Void material has neither mass nor strength.

\subsubsection{Interaction}

The general contact algorithm also enforces contact between Eulerian materials and Lagrangian surfaces. This algorithm automatically compensates for mesh size discrepancies to prevent penetration of Eulerian material through the Lagrangian surface.

The Eulerian-Lagrangian contact formulation is based on an enhanced immersed boundary method. In this method the Lagrangian structure occupies void regions inside the Eulerian mesh. The contact algorithm automatically computes and tracks the interface between the Lagrangian structure and the Eulerian materials. A great benefit of this method is that there is no need to generate a conforming mesh for the Eulerian domain. In fact, a simple regular grid of Eulerian elements often yields the best accuracy. 
If the Lagrangian body is initially positioned inside the Eulerian mesh, the Lagrangian body pushes material out of the Eulerian elements that it passes through during the analysis, and they become filled with void. Similarly, Eulerian material flowing toward the Lagrangian body is prevented from entering the underlying Eulerian elements. This formulation ensures that two materials never occupy the same physical space.

If the Lagrangian body is initially positioned outside the Eulerian mesh, at least one layer of void Eulerian elements must be present at the Eulerian mesh boundary. This creates a free surface on the Eulerian material inside the Eulerian mesh boundary and provides a source for void material to replace Eulerian material that is driven out of interior elements.

Eulerian-Lagrangian contact also supports failure and erosion in the Lagrangian body. Lagrangian element failure can open holes in a surface through which Eulerian material may flow. When modeling erosion of a solid Lagrangian body, the interior faces of the solid body must be included in the contact surface definition, see chapter 5 for details.

Eulerian-Lagrangian contact constraints are enforced using a penalty method, where the default penalty stiffness parameter is automatically maximized subject to stability limits.

Eulerian-Lagrangian contact supports thermal interactions when using coupled temperature-displacement Eulerian element EC3D8RT in a dynamic coupled thermalstress analysis.

\subsubsection{Load}

A gravity load is applied to the entire model, which including Eulerian and Lagrangian parts, through acceleration due to gravity of $9.8 \mathrm{~m} / \mathrm{s}^{2}$ in the normal direction.

\subsubsection{Boundary conditions}

By default, Eulerian material can flow freely into and out of the Eulerian domain through mesh boundaries. One can use prescribed velocity or acceleration conditions on Eulerian nodes to control material flow. Prescribed velocity or acceleration is implemented in an Eulerian frame, so material velocity will reach the prescribed value as the material passes the Eulerian node. The boundary conditions for Eulerian and Lagrangian parts are shown in FIG.2.21.

\subsubsection{Initial conditions}

One can apply initial conditions to Eulerian nodes and elements in the same way that they are used for Lagrangian nodes and elements. In addition, most Eulerian analyses require the initialization of Eulerian material.

By default, all Eulerian elements are initially void. To fill an Eulerian element, one must define an initial volume fraction, which relates directly the initial topology of Eulerian material, for each available material instance. Material is filled until a volume fraction of 1.0 is reached; any excess material is ignored. For elements that are partially filled or 


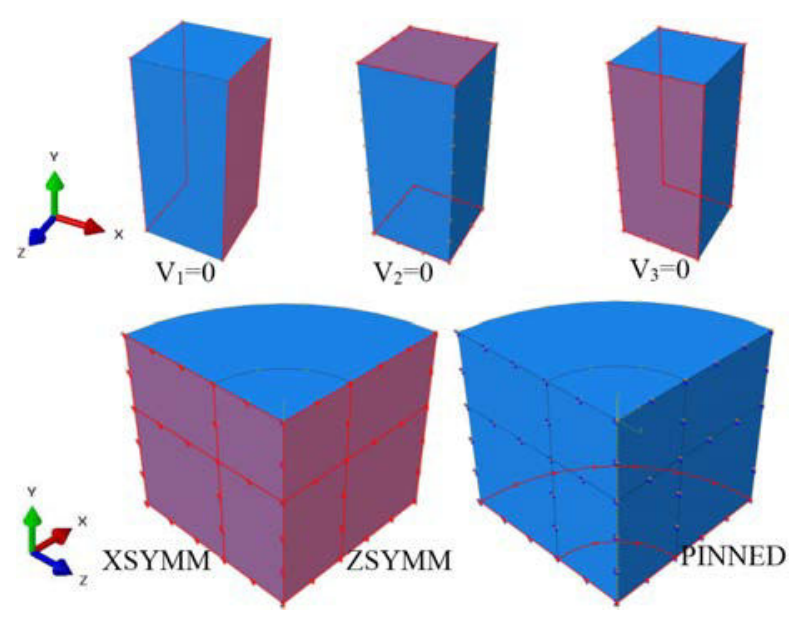

Figure 2.21: Boundary conditions for the 3D CEL model.

filled with multiple materials, the exact geometric composition of the material within the element is not known; ABAQUS interpolates the material volume fractions from adjacent elements to estimate the material boundaries within the element. The initial conditions apply only at the beginning of an analysis; during the analysis the materials deform according to the applied loads, and the volume fractions are recalculated accordingly.

Assigning materials to Eulerian part instances The volume fraction tool creates discrete fields specifically for use in material assignment predefined fields by performing a Boolean comparison between an Eulerian part instance and a second part instance (the reference part instance) that intersects the Eulerian instance. The comparison determines where the two part instances overlap, then assigns each element in the Eulerian instance a volume fraction based on the percentage of the element that is also occupied by the reference instance. The volume fraction is specified as a decimal between zero and one. The discrete field that is created by the volume fraction tool can be used to assign material instances to the Eulerian part instance. About how to use the volume fraction tool, one could see Appendix G for detailed information.

\subsubsection{Mesh}

The Eulerian method is implemented in the multi-material thermally coupled element type EC3D8RT. The underlying mechanical response formulation of these elements is based on the Lagrangian C3D8R element with extensions to allow multiple materials and to support the Eulerian transport phase. The formulation applies the same strain to each material in the element, then allows the stress and other state data to evolve independently within each material. These stresses are combined using volume fraction data to create element averaged values, which are integrated to obtain nodal forces. Similarly, the thermal response formulation for the thermally coupled element is based on the Lagrangian element C3D8RT with the extension to allow multiple materials with different thermal 
properties and to support temperature advection. All the materials have the same temperature, and the thermal properties are volume averaged before being used in solving one single heat transfer equation for the multi-material model. The Eulerian elements use viscous hourglass control.

\subsubsection{Results and discussion}

The CEL method permits to avoid problems of excessive mesh distortion and unrealistic deformed shape which is a straightforward result of the physically enhanced modeling of fluid-like particle by the CEL particle model, as shown in FIG.2.22. Noting that the material jet still occurs at the substrate surface, but it does not affect the completion of calculation. The results of the Eulerian analysis (FIG.2.22a and FIG.2.23a) should be interpreted differently than those with a Lagrangian method. Any result based on nodal displacements is meaningless in an Eulerian model because the Eulerian part is fixed and rigid. Therefore, the equivalent plastic strain is computed as a volume fraction weighted average of all materials in the element, and definitely this volume average value is significantly lower than that of the Lagrangian analysis with and without adaptive remeshing. This explanation also applies to the temperature distribution of particle as FIG.2.23a shows.

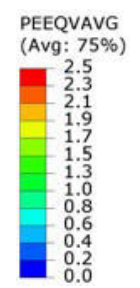

(a)
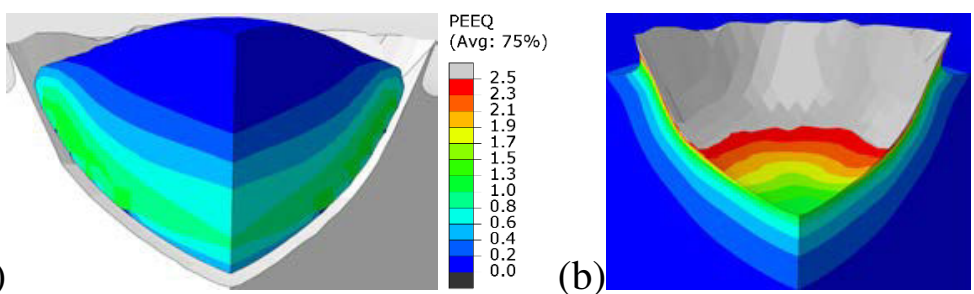

Figure 2.22: Contours of (a) volume average equivalent plastic strain of Al particle and (b) equivalent plastic strain of $\mathrm{Al}$ substrate by using the CEL numerical approach at 700 $\mathrm{m} / \mathrm{s}$.
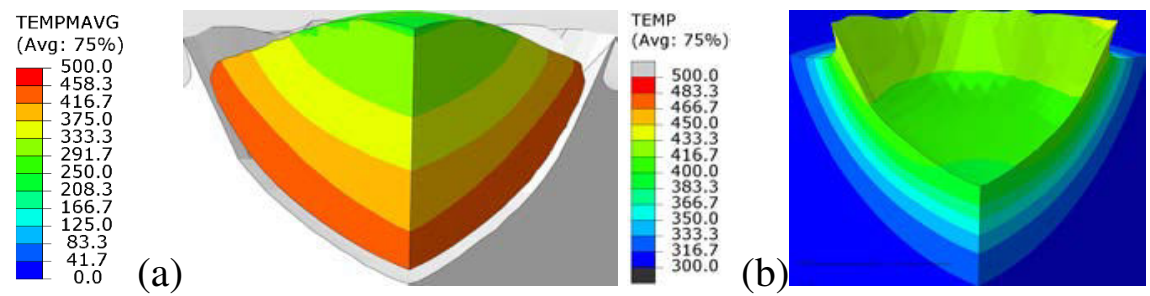

Figure 2.23: Contours of (a) volume average temperature of $\mathrm{Al}$ particle and (b) temperature of Al substrate by using the CEL numerical approach at $700 \mathrm{~m} / \mathrm{s}$.

FIG.2.24 shows the development of plastic strain and temperature in the key elements at the interface which undergoes the highest amount of deformation/temperature. For substrate, the plastic strain increases rapidly at a rate of $10^{6} s^{-1}$, before reaching its final 
value of about 5.5 it shows a further increase of strain at about $30 \mathrm{~ns}$. For the particle, first, the volume average of plastic strain is significantly lower than that of substrate; second, the time history of temperature is not the true time history of materials, but the time history of one integration point; third, when the materials have not pass the Eulerian mesh or have left the Eulerian mesh, the values become zero. It is hard to trace the history behavior of materials, and that's one of the shortcomings of CEL approach.

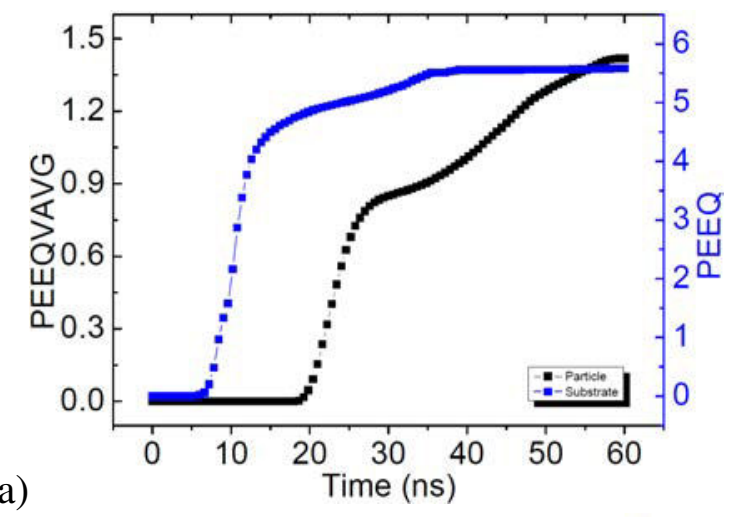

(a)

(b)

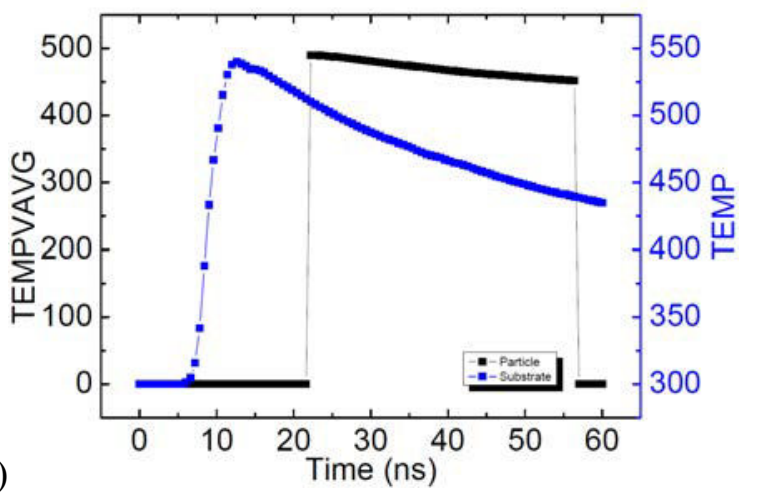

Figure 2.24: Time histories of (a) equivalent plastic strain (PEEQ) and (b) temperature of $\mathrm{Al} / \mathrm{Al}$ by using the CEL approach at $700 \mathrm{~m} / \mathrm{s}$.

\subsection{Discussion}

\subsubsection{Comparison of four numerical approaches}

The evolution of normalized nodal displacement - Displacement $/ D_{p}$ by using the ALE approach are show in FIG.2.25. Results for four distinct nodes, which are at the top (node 4), center (node 3) and bottom (node 2) of the particle, and the initial contact point of the substrate (node 8), are given. It can be seen from FIG.2.25 that, comparing the displacement history of bottom, center and top of particle at $700 \mathrm{~m} / \mathrm{s}$, the difference between the displacement at the top and at the center of the particle is much smaller than the gap between displacements at the center and at the bottom of the particle, which implies that 
during the impact the larger deformation is mainly localized at the bottom half of particle. After about $40 \mathrm{~ns}$, the separation of particle from substrate starts based on the difference between the displacement at bottom of particle (red curve) and displacement at contact point of substrate (black curve). The nodal displacement at the initial contact point of substrate (indentation of substrate) does not recover back to zero after separation of particle from the plastically deformed substrate, indicating the permanent plastic indentation occurs in the substrate.

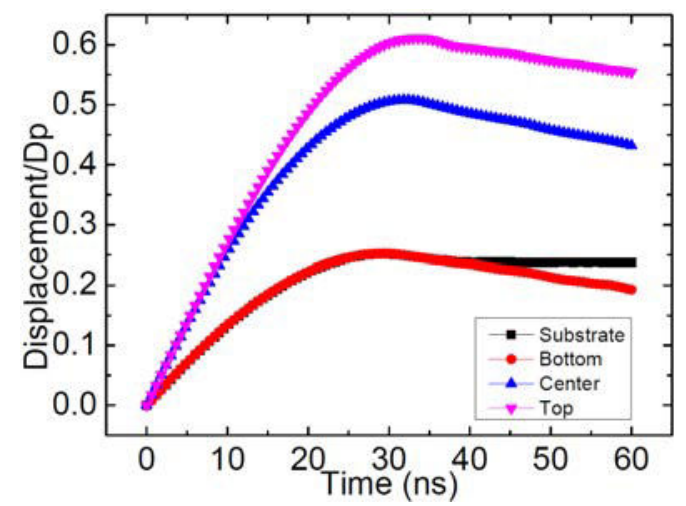

Figure 2.25: Time histories of normalized displacement of specific nodes by using the ALE approach.

The time histories of normalized kinetic energy by using the four approaches are shown in FIg.2.26a. The rebound kinetic energy is much less than the initial kinetic energy, which more than $98 \%$ kinetic energy is dissipated into internal energy. The rebound kinetic energy has slightly $0.3 \%$ increase since $30 \mathrm{~ns}$, and this energy match the recoverable elastic energy. Although the calculation in Lagrangian reference frame is aborted earlier, the trend of normalized kinetic energy at first $20 \mathrm{~ns}$ still agrees well with ALE and CEL cases. The SPH approach has a clear deviation from the other three approaches due to the different analysis procedure used.

The time histories of normalized indentation by using four approaches are shown in FIG.2.26b. Again, Lagrangian and ALE and CEL obtain accordant tendency. The substrate has a $1 \%$ recoverable displacement until the end of calculation. Without considering the thermal softening, the SPH particle is stiffer than the other three particles, and thus the substrate undergoes deeper indentation.

A comparison of the stable time increment time histories for the four analysis cases is shown in FIg.2.27a. The Lagrangian approach has the smallest stable time increment as a result of the large changes in element aspect ratio. With adaptive meshing technique, the stable time increment increases at the last half the analysis process. Without considering the thermal solution response on the stability of SPH approach, the stability limit is only decided by the mechanical response, and thus higher than the other three cases. For CEL approach, without any mass scaling, the stable time increment for the problem is observed to reduce dramatically at the beginning, and then keep a relatively constant value till the completion of calculation. 
(a)

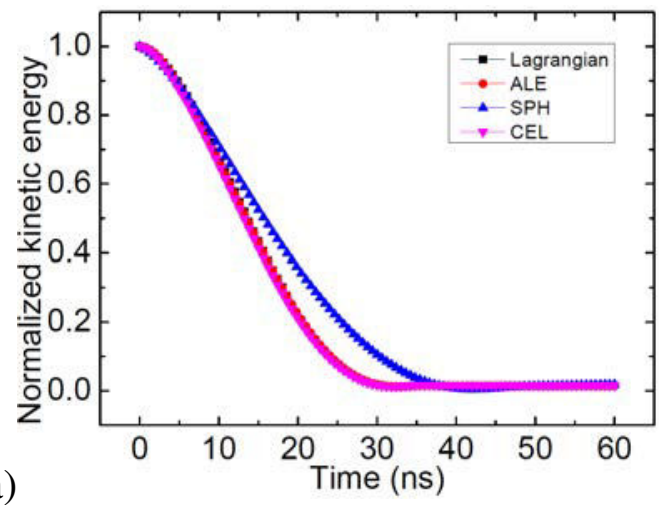

(b)

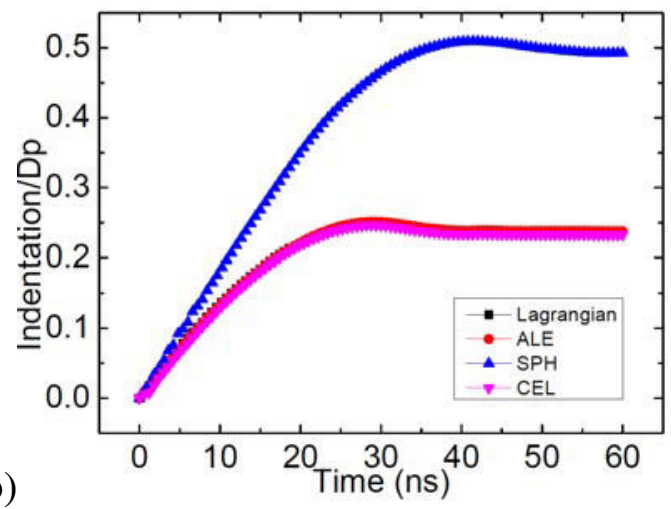

Figure 2.26: Time histories of (a) normalized kinetic energy and (b) normalized displacement of substrate center by using the four approaches at $700 \mathrm{~m} / \mathrm{s}$.

'Artificial' strain energy associated with constraints is used to remove singular modes (such as hourglass control). If the artificial strain energy is excessive, too much strain energy may be going into controlling hourglassing deformation. To determine what amount of artificial energy is considered excessive, one could compare the artificial strain energy (ALLAE) to the other internal energy (ALLIE) as shown in FIG.2.27b. As a general rule, the ratio of ALLAE to ALLIE should be less than 5\%. Neglecting the initial ratios close to 0.00 second, which is basically noise generated by dividing a very small number by another, all the ratios of these four approaches are lower than $4 \%$. The inherent hourglass control is sufficient to prevent hourglass from being a problem. The mesh size is fine enough to stop the propagation of zero-energy modes, which potentially produce incorrect results.

(a)

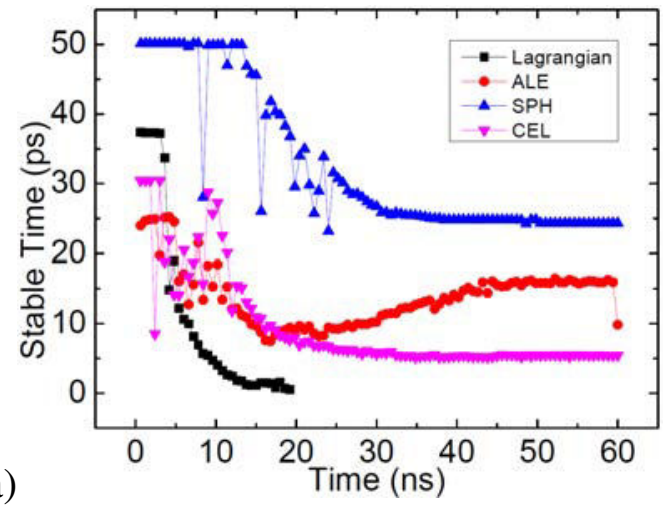

(b)

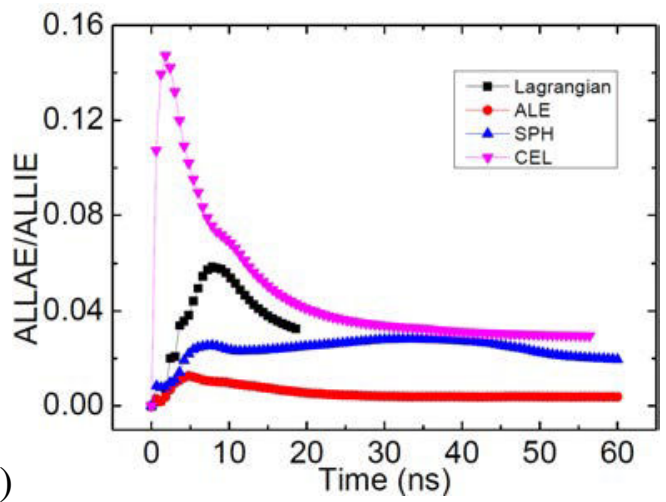

Figure 2.27: Time histories of (a) stable time increment and (b) artificial strain energy by using the four approaches at $700 \mathrm{~m} / \mathrm{s}$.

The relative computational costs of these four approaches are listed in TAB.2.3. The pure Lagrangian analysis is most efficiency, and the SPH approach is rather time consuming. 


\begin{tabular}{|c|c|c|c|c|c|c|}
\hline $\begin{array}{l}\text { Analysis } \\
\text { case }\end{array}$ & $\begin{array}{l}\text { Element } \\
\text { type of } \\
\text { Particle }\end{array}$ & $\begin{array}{l}\text { Element } \\
\text { quan- } \\
\text { tity of } \\
\text { Particle }\end{array}$ & $\begin{array}{l}\text { Element } \\
\text { type of } \\
\text { Substrate }\end{array}$ & $\begin{array}{l}\text { Element } \\
\text { quantity } \\
\text { of Sub- } \\
\text { strate }\end{array}$ & $\begin{array}{l}\text { CPU } \\
\text { Time (s) }\end{array}$ & $\begin{array}{l}\text { Relative } \\
\text { Cost per } \\
\text { Incre- } \\
\text { ment per } \\
\text { Element }\end{array}$ \\
\hline Lagrangian & CAX4RT & 1050 & CAX4RT & 14400 & 87.9 & 1.00 \\
\hline ALE & CAX4RT & 1050 & CAX4RT & 14400 & 58 & 1.15 \\
\hline SPH & PC3D & 7989 & C3D8R & 255000 & 599.2 & 1.62 \\
\hline CEL & EC3D8RT & 18000 & C3D8RT & 613100 & 5849.3 & 1.56 \\
\hline
\end{tabular}

Table 2.3: Comparison of computational cost by using four approaches.

\subsubsection{Typical impact behaviors of cold sprayed particle and substrate}

\subsubsection{Deformation shape}

In the whole process, the deformation changes from purely elastic to elastic-plastic then to fully plastic, which is the common phenomena for most engineering materials in contact.

- Elastic phase: the deformations of both contacting bodies are elastic and the solution of Hertz theory holds.

- Elastic-plastic phase: this is a transitional phase from purely elastic to fully plastic when neither plastic nor elastic strains dominate over the other. It begins once yield occurs in the body with a lower yield strength and terminates by the fully plastic phase. The yield will be first developed inside the body at some distance from the center of the contact surface and a small amount of plastic flow occurs within the larger elastic hinterland. As the load and contact pressure continue to be increased, the plastic zone grows.

- Fully plastic phase: this becomes dominant once the plastic zone reaches the surface and the material surrounding the contact area undergoes plastic deformation.

The case of $\mathrm{Al} / \mathrm{Al}$ with impact velocity of $500 \mathrm{~m} / \mathrm{s}$ is selected for studying the impact behaviors of cold sprayed particle and substrate. The impact time of Al particle is divided into two parts: the deformation up to maximum compression which last $24.6 \mathrm{~ns}$, followed by the rebound. The evolution of the Von Mises stress by using the Lagrangian numerical approach at various instants during $60 \mathrm{~ns}$ is shown in FIG.2.28.

For Al material, the flow stress increases as a result of plastic deformation and working hardening. As the FIG.2.28a to FIG.2.28c show, the deformation process during the contact induces an increase in the Von Mises stress, which the stress wave propagates to the boundary of substrate. The stress distribution in FIG.2.28d indicates that the particle has rebounded, but the two bodies are still in contact. The unloading process leads the substrate has two lower strength regions beneath the contact center. These two regions disappear over time as the FIg.2.28e and FIg.2.28f show. At $40 n s$, the particle has already completely detached from the substrate, and the inside of particle also appears the lower strength region (FIG.2.28e). A clear gap between particle and substrate is seen 
at the end of calculation (FIG.2.28f), and the residual stresses remain inevitably in the particle and substrate after the impact loading has been removed.

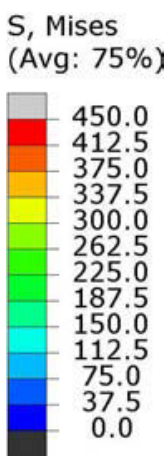

S, Mises (Avg: $75 \%$ )

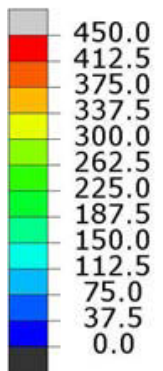
0.0 (a) 4
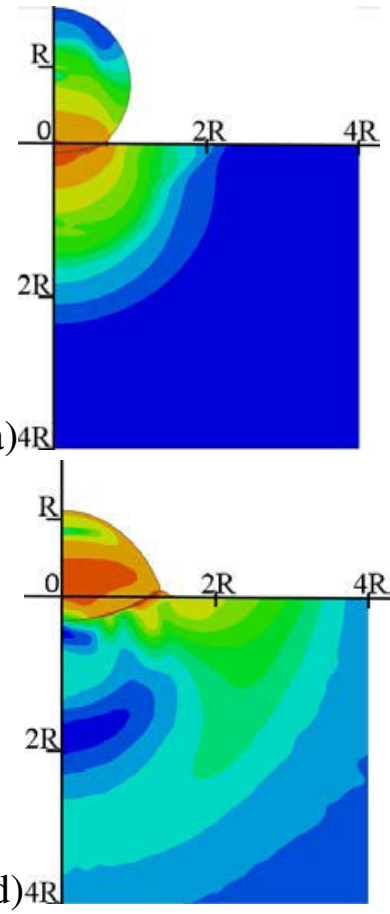

(b)

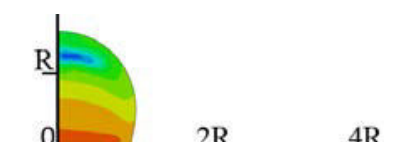

(e) 4
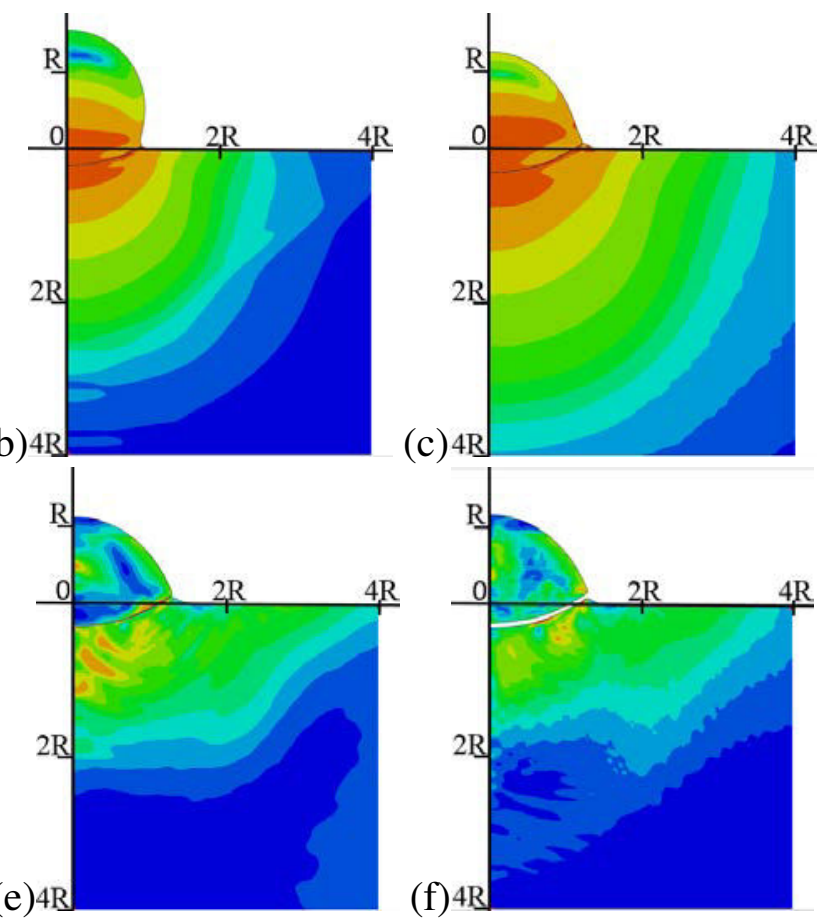

(f) 4

(a) $5 n s$; (b) $10 n s$; (c) $20 n s$; (d) $30 n s$; (e) $40 n s$; (f) $60 n s$

The evolution of the mean stress, in ABAQUS which called PRESSURE, at various instants during $60 n s$ is shown in FIG.2.29. Note that the positive stresses represent the compressive stresses in ABAQUS/Explicit, and thus the negative ones represent the tensile stresses. At $5 n s$, the high velocity impact has already induced a large compressive stress region near the contact area. This compressive stress region expands over time, meanwhile, the tensile stress region within the upper particle contracts over time. After $10 \mathrm{~ns}$, the tensile stresses also occur beneath the substrate surface at a depth of $3 \mathrm{R}$ on the axis of symmetry. This tensile stress region becomes larger and larger over time, but never reaches to the substrate surface until the particle rebounds from the substrate. As the FIG.2.29f shows, at the position of the maximum equivalent plastic strain situated and the edge of contact, the tensile stress region occurs. However, these tensile stress regions are localized, from contact center to the depth of $2 \mathrm{R}$ it still remains a large compressive region. This compressive residual stress is good for improving fatigue resistance of material, and that will be discussed in chapter 4 in detail.

The corresponding evolution of equivalent plastic strain are shown in FIG.2.30. The results presented in FIG.2.30 show that the outer contact region undergoes severer plastic deformation than that of the center of contact region. During the contact process, the contact region is larger and larger based on the motion of maximum equivalent plastic strain (FIG.2.30a to FIG.2.30c), and the piling-up of substrate surface material around the 


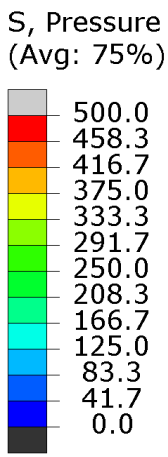

$\mathrm{S}$, Pressure (Avg: 75\%)

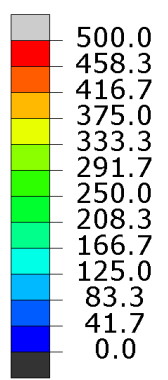

(d)

(a) $4 \mathrm{R}$

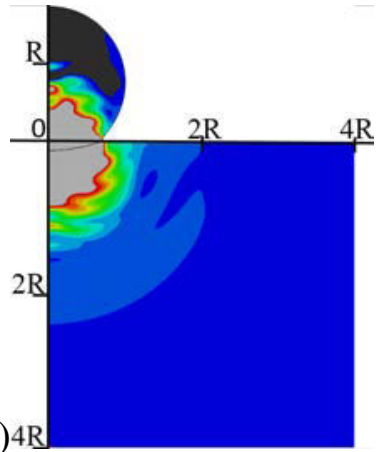

(b) 4
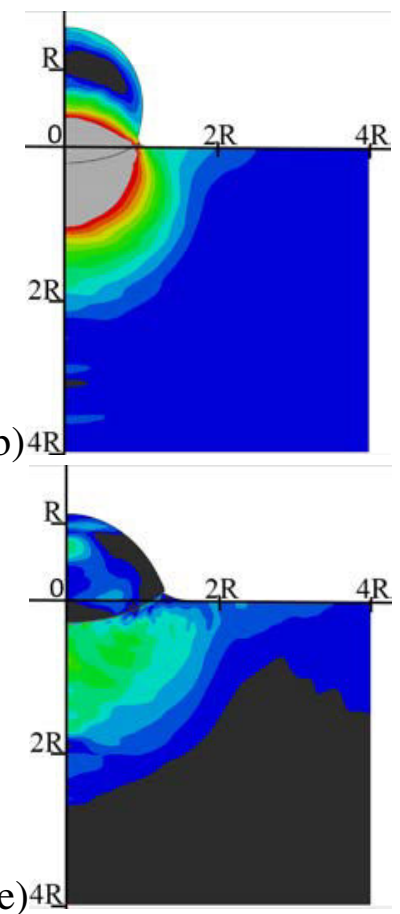

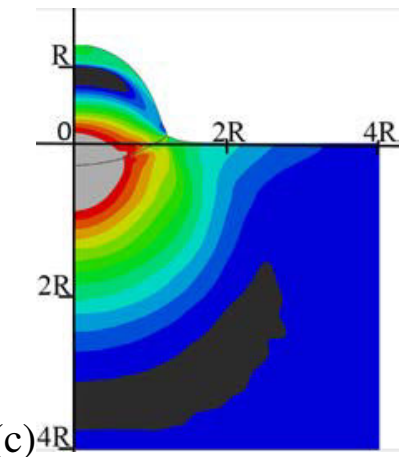

$(\mathrm{c}) 4$

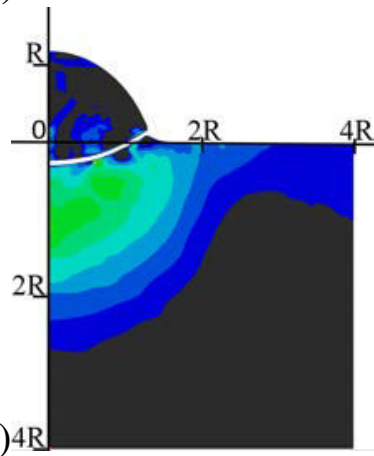

Figure 2.29: Evolution of mean stress of $\mathrm{Al} / \mathrm{Al}$ at $500 \mathrm{~m} / \mathrm{s}$. (a) $5 \mathrm{~ns}$; (b) $10 \mathrm{~ns}$; (c) $20 \mathrm{~ns}$; (d) $30 n s$; (e) $40 n s$; (f) $60 n s$.

contact region become more and more apparently. The contact region does not change too much after the rebounding of particle (FIG.2.30d to FIG.2.30f), due to permanent plastic deformation is occurred both in the particle and substrate and the recoverable elastic energy is relatively small compared to the plastic deformation energy. The deformation is occurred mostly in the bottom of particle and substrate surface region with a few micrometers of depth, therefore, the deformation is localized due to the higher impact loading and shorter contact time. It can be seen from Fig.2.30d to Fig.2.30f that the overall plastic strain distributions during the restitution process are essentially identical, which qualitatively implies that the process of the restitution is essentially elastic.

The strain rates along the horizontal direction of contact surface of particle and substrate at various instance are presented in FIG.2.31. It is clear to see that the higher strain rate up to $0.5 \times 10^{6} s^{-1}$ occurs at $5 \mathrm{~ns}$ for both the particle and substrate. Then it decreases below $1 \times 10^{5} s^{-1}$ after $15 n s$, and reaches the lower limit at $30 n s$. Note that at $30 n s$ the strain rates do not become zero, actually, they still have the values of about $10^{3} s^{-1}$, and the strain rate of particle is still higher than that of substrate. Even the strain rate is very high, it is just situated at very local area rather than whole contact area.

\subsubsection{Contact pressure}

FIG.2.32a shows the contact pressure distribution along the horizontal direction of substrate surface at various instants during the impact case of Al/Al with $500 \mathrm{~m} / \mathrm{s}$. A 

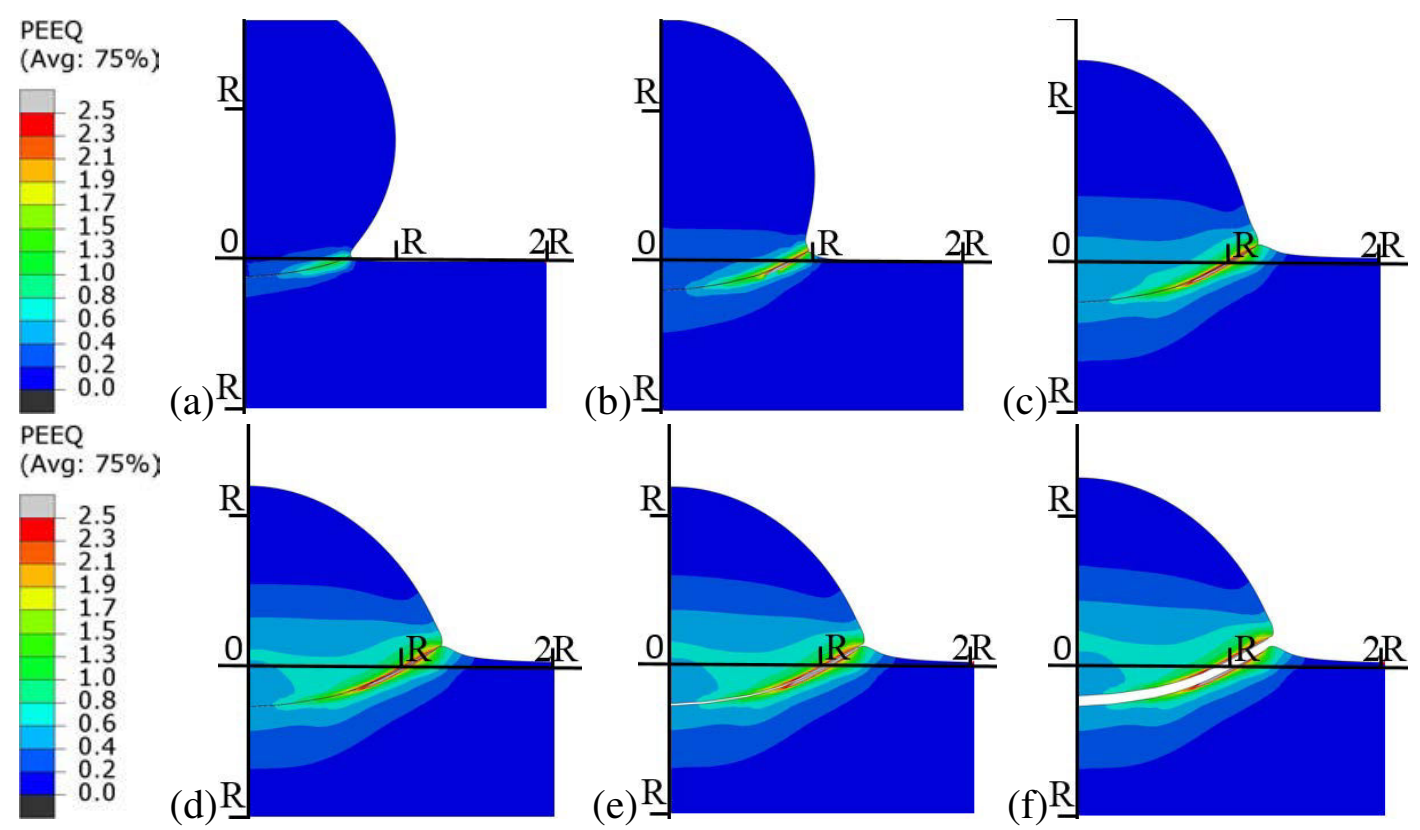

Figure 2.30: Evolution of equivalent plastic strain (PEEQ) of $\mathrm{Al} / \mathrm{Al}$ at $500 \mathrm{~m} / \mathrm{s}$. (a) $5 \mathrm{~ns}$; (b) $10 n s$; (c) $20 n s$; (d) $30 n s$; (e) $40 n s$; (f) $60 n s$.

flattening of the pressure distribution is apparent since $10 \mathrm{~ns}$, and the pressure decreases over time and become zero for all over the contact area at $40 \mathrm{~ns}$. The peak of pressure distribution appears at $20 \mathrm{~ns}$ where is located in the edge of contact area. The rebounding of particle starts from the contact center, and then extends to the edge of contact area, so this peak does not disappear until the whole body of particle is completely separated from the substrate.

The evolution of shear stress along the horizontal direction of substrate surface is shown in FIG.2.32b at various instants. The direction of shear stress switches after 10 $n s$, this may due to the different temperature distribution along the two sides of contact surface as the FIG.2.33 shows. It is clearly seen that after $10 \mathrm{~ns}$, the temperature along the particle side is significantly higher than that of substrate side, which means the Al material of particle near the contact area becomes relatively softer than the Al material of substrate in the other side of contact surface, and thus relative motion between the particle and substrate occurs. When the rebounding of particle happens, comparing the curve of $20 n s$ and $30 n s$ the shear stress increases over the time, and the maximum shear stress is always occurred in the position of the edge of contact area.

\subsubsection{Contact force - displacement relationship}

The contact force-displacement relation is crucial for understanding the impact behavior of elastic-plastic particles. The analytic derivation of the contact force-displacement relation should be based on the determination of both the contact pressure distribution and the relative approach and the contact radius. For fully plastic contact with finite plastic 

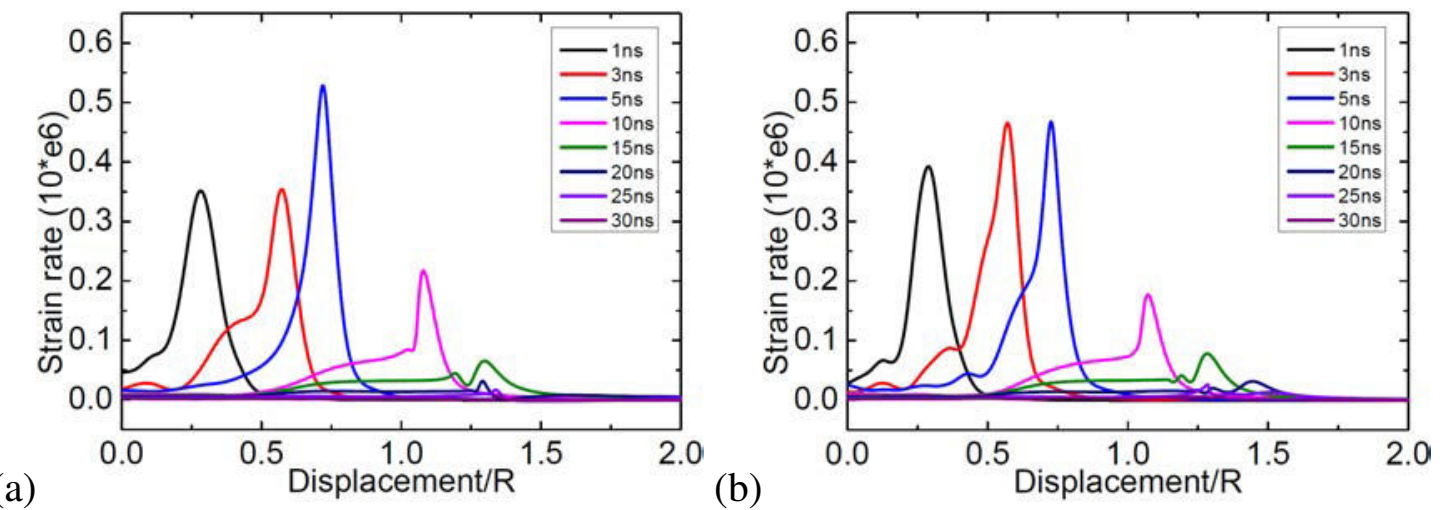

Figure 2.31: Evolution of strain rate along the horizontal direction of contact surface of (a) Al particle and (b) Al substrate at $500 \mathrm{~m} / \mathrm{s}$.

(a)

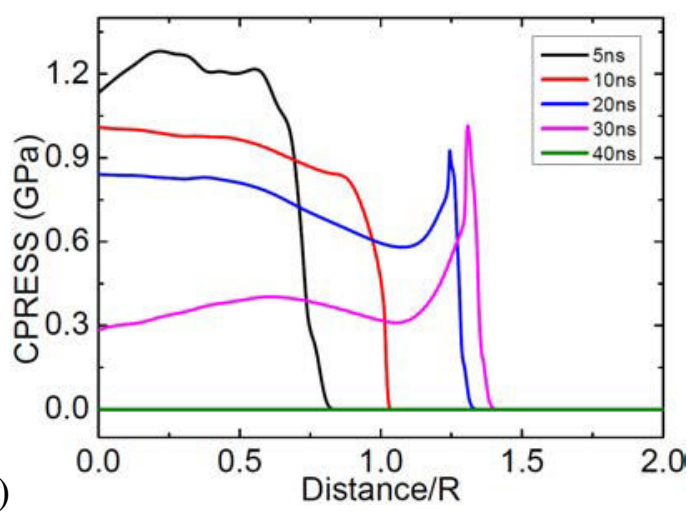

(b)

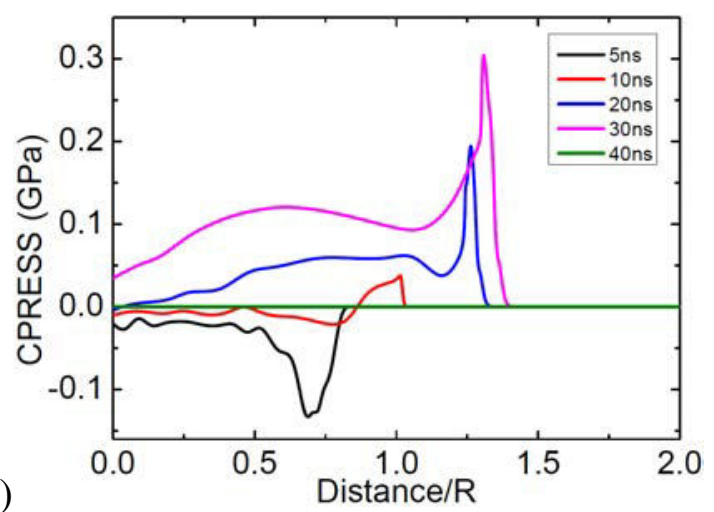

Figure 2.32: Evolution of (a) contact pressure (CPRESS) and (b) frictional shear stress (CSHEAR) of Al substrate impacted by an $\mathrm{Al}$ particle at $500 \mathrm{~m} / \mathrm{s}$.

deformation region, like cold spray impact, the mean contact pressure no longer remains constant, instead, it decreases with the increases contact size as the FIG.2.32a shows. The solution for this case becomes more complicated and has not been solved yet. Thus here, only the evolution of contact force applied at the substrate surface and the contour of contact surface are presented in FIG.2.34.

During the loading process $(0-24.6 \mathrm{~ns})$, the maximum contact force increases over time, and the position related to the peak value moves far away from the contact center, which implies the expanding of contact area. The contact force applied in the area where around the contact center decreases after $20 n s$ even the two bodies are still in contact with each other; but it drops to zero fast since the rebounding of particle commences. During the unloading process, the peak value of contact force decreases $38.52 \%$ but the position of that only change $3.56 \%$ following the enlarging direction. Assuming the $R_{c}$ is the radius of contact area, then the maximum contact force appears between $0.65 R_{c}$ and $0.73 R_{c}$ at various instants as FIG.2.35 shows.

The results presented in FIG.2.34b do not only show the expanding of contact surface 

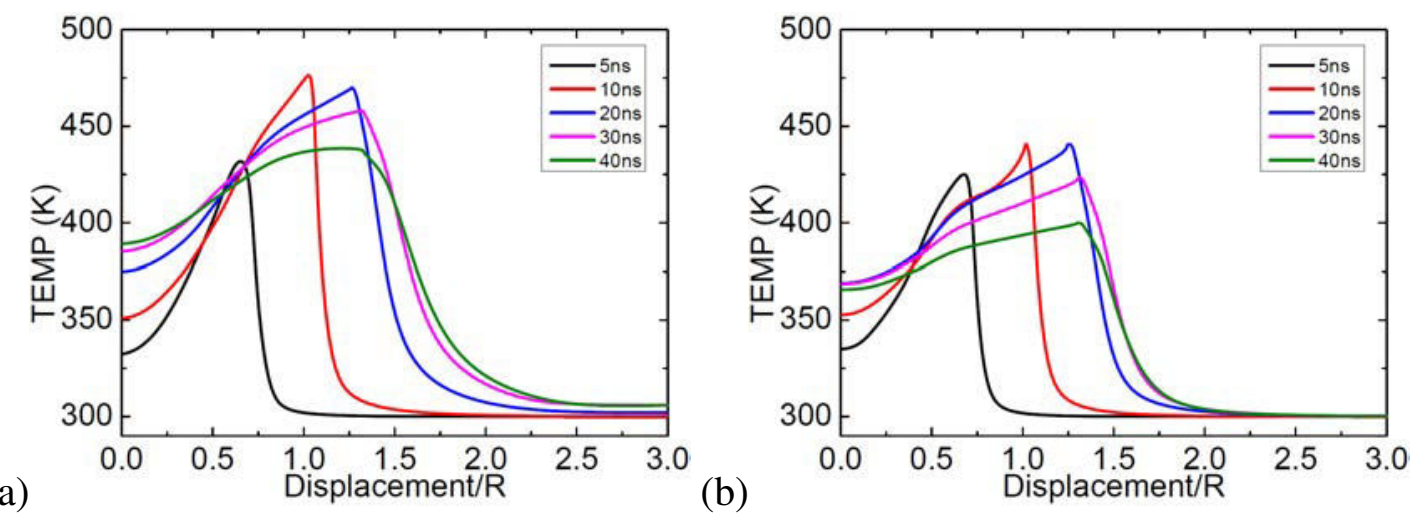

Figure 2.33: Evolution of temperature (TEMP) along the horizontal direction of contact surface of (a) Al particle and (b) Al substrate at $500 \mathrm{~m} / \mathrm{s}$.

along the radius direction, but also that along the depth direction. The deformation ratio - $R_{c} / h$ - presented in FIG.2.35 shows that the deformation is highly non linear. $h$ is the indentation depth of substrate. Due to the first instance of impact, the contact surface component along the horizontal direction moves faster than that of longitudinal direction, thus at $5 n s$, it has the highest value of 7.4. Then the deepen rate is slightly higher than the widening rate, thus the deformation ratio decreases over time. When the particle completely separated from the substrate, the indentation of contact center recovers about $3.8 \%$, which contribute slightly to rise of deformation ratio at $30 \mathrm{~ns}$.

(a)

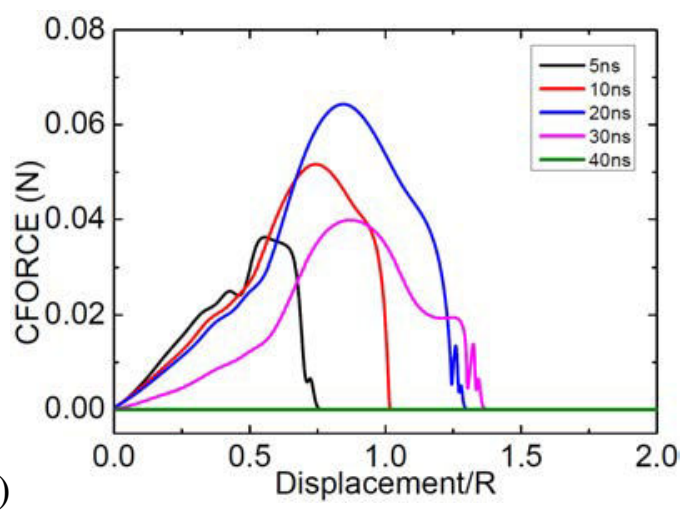

(b)

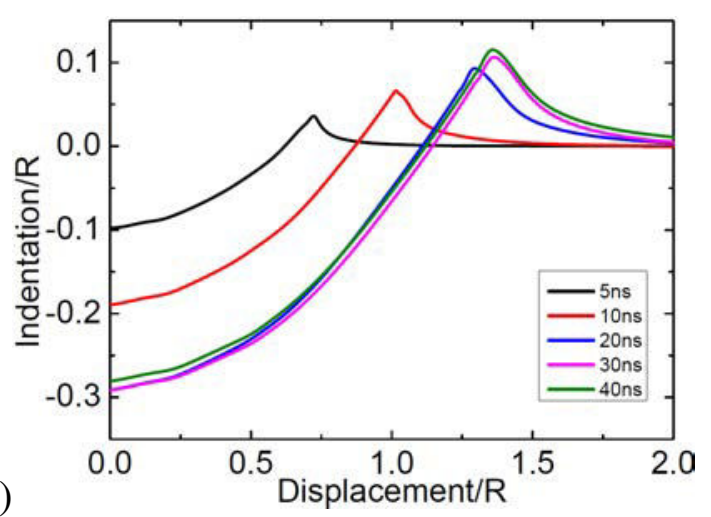

Figure 2.34: Evolution of (a) contact force (CFORCE) and (b) normalized displacement of $\mathrm{Al}$ substrate impacted by an $\mathrm{Al}$ particle at $500 \mathrm{~m} / \mathrm{s}$.

\subsection{Summary}

The Lagrangian approach is computational efficient and permits to incorporate complex material models. But the mesh structure could become severely distorted in the 


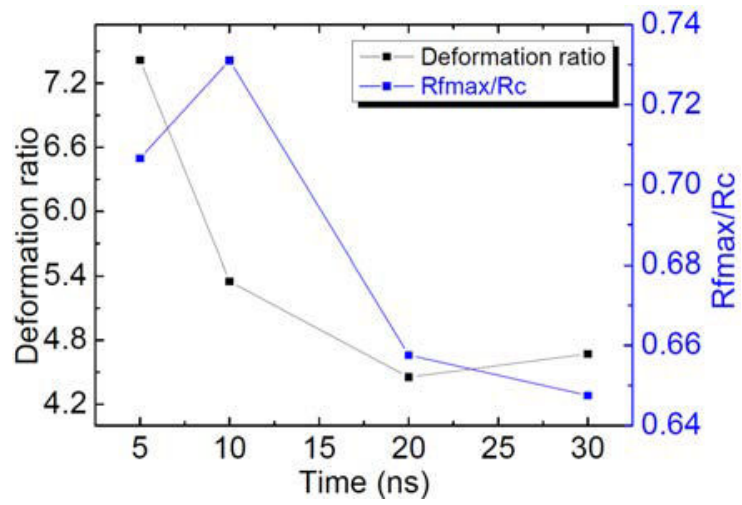

Figure 2.35: Deformation ratio and the position related to the maximum contact force of $\mathrm{Al}$ substrate impacted by an $\mathrm{Al}$ particle at $500 \mathrm{~m} / \mathrm{s}$.

deformed region, which can lead to numerical backside effects on the convergence and accuracy.

The ALE approach could reduce or eliminate difficulties caused by severe mesh distortion and allows a calculation to continue efficiently. However, an additional computation cost occurs due to the adaptive meshing. It should be used very cautiously, and or should be very careful with the choice of parameters (Frequency and Intensity, etc.).

For the SPH approach, they are less accurate in general than Lagrangian finite element analyses. Smoothed particle hydrodynamic analyses are effective for applications involving extreme deformation. For example, tracking fragments from primary impacts through a large volume until secondary impact occurs can be very expensive in a coupled Eulerian-Lagrangian analysis but comes at almost no additional cost in a smoothed particle hydrodynamic analysis.

In higher deformation regimes Coupled Eulerian-Lagrangian analyses are generally more accurate and more robust. Hence we choose it for the investigation of next step, the simulation results that will be presented in chapter 3 to chapter 5 are all obtained by using the CEL numerical approach.

The phenomena characteristics of a typical cold spray impact are shown in the last section of this chapter, illustrating the deformation mechanisms of cold sprayed particle and substrate. The conforming contact between particle and substrate makes the size of contact area is comparable with the significant dimensions of the particle body. The deformation process is dynamic with a high strain rate, and the piling up or sink up of materials is too significant to be neglected, thus it is difficult to obtain an analytic solution of contact stress using a quasi-static approach. Only the evolution of mean stress, strain rate, contact pressure, contact shear stress, contact force were shown in order to achieve a qualitative understanding about cold spray contact process. 


\section{Chapter 3}

\section{Simulation of single impact}

The impact can generally be categorized into normal impact and oblique impact, according to whether the direction of initial velocity coincides with the normal direction of the initial contact plane. Simulation results are presented first for normal impact in this chapter, and that for oblique impact will be presented in Chapter 5. The capability of the CEL numerical approach in modeling the Cold Spray deposition process is verified through a systematic parametric study, including impact velocity, initial particle temperature, friction coefficient and materials. The simulation results by using the CEL numerical approach are compared with the experimental results published in the literature. The analysis of equivalent plastic strain and temperature under the different circumstances allows us to deeply understand phenomena involved in high velocity impact phenomenon. In order to study the relationship between kinetic energy and deposition, the restitution coefficient is discussed at the end of this chapter.

\section{Contents}

3.1 Simulation outline $\ldots \ldots \ldots \ldots \ldots \ldots \ldots$

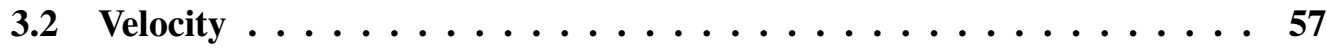


3.2.1 Deformation shape . . . . . . . . . . . . . . . 57

3.2.2 Evolution of the strain and temperature . . . . . . . . . . 59

3.3 Temperature ......................6 60

3.3.1 Deformation shape . . . . . . . . . . . . . . . 60

3.3.2 Evolution of the strain and temperature . . . . . . . . . . 61

3.4 Friction coefficient $\ldots \ldots \ldots \ldots \ldots \ldots$

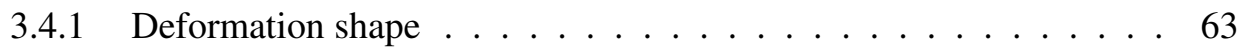

3.4 .2 Evolution of the strain and temperature . . . . . . . . . 63

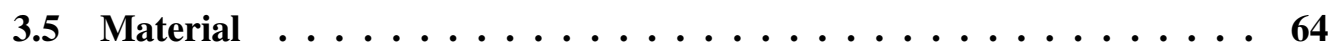

3.5.1 Deformation shape . . . . . . . . . . . . . 64

3.5.2 Evolution of the strain and temperature . . . . . . . . . . 65

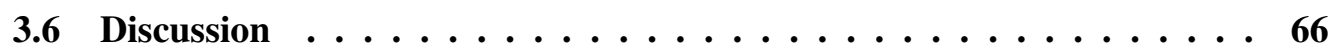

3.6.1 Energy dissipation and restitution coefficient $\ldots \ldots \ldots 66$

3.7 Summary . . . . . . . . . . . . . . . . . 69 


\subsection{Simulation outline}

Investigation of particle impact on the substrate surface is a key problem whose solution could help to understand the mechanisms of coating formation. This chapter pays main attention to the interaction of the so-called 'single particle' with the substrate surface. During impact, the deformation behavior of the contacting body generally depends on the nature of solid bodies, such as elastic, elastic-perfectly plastic, elastic-plastic with work hardening, visco-elastic and visco-plastic solids. In this study, we only consider the impact of elastic-plastic with isotropic work hardening. ТАВ.3.1 gives the brief outline of the parametric study, focusing on the parameters of impact velocity, initial temperature of the particle, friction coefficient and materials. The geometry, interaction, analysis procedure, boundary conditions and mesh are the same as discussed in the previous chapter. The materials [KIN 10] used in this chapter are displayed in TAB.3.2 and Appendix B. There are two paths, which path 1 along the X-direction and path 2 along the Y-direction, for observing the evolution of plastic strain and temperature as illustrated in FIG.3.1.

\begin{tabular}{|l|c|c|l|l|}
\hline Section & Parameter & Size $(\mu m)$ & Material & Velocity $(m / s)$ \\
\hline Sec. 3.2 & Velocity & 25 & $\mathrm{Al} / \mathrm{Al}$ & $700-780-840$ \\
Sec. 3.3 & Temperature & 25 & $\mathrm{Cu} / \mathrm{Al}$ & 600 \\
Sec. 3.4 & Friction coefficient & 20 & $\mathrm{Cu} / \mathrm{Cu}$ & 500 \\
Sec. 3.5 & Material & 20 & $\mathrm{Al} / \mathrm{Al}-\mathrm{Cu} / \mathrm{Cu}-$ & 500 \\
& & & $\mathrm{Al} / \mathrm{Cu}-\mathrm{Cu} / \mathrm{Al}$ & \\
\hline Section & Temperature $(K)$ & Contact angle $\left(^{\circ}\right)$ & Friction & Reference \\
\hline Sec. 3.2 & 300 & 90 & 0.3 & [MAN 11c] \\
Sec. 3.3 & $473-673-873$ & 90 & 0.3 & [KIN 10] \\
Sec. 3.4 & 300 & 90 & $0.2-0.3-0.5$ & [LI 09a] \\
Sec. 3.5 & 300 & 90 & 0.3 & [KIN 08] \\
\hline
\end{tabular}

Table 3.1: Calculation plan for investigating the effects of parameters.

\subsection{Velocity}

\subsubsection{Deformation shape}

The deformation behaviors of $\mathrm{Al}$ impacted particle at different impact velocities obtained from experiments [MAN 11b] and modeled by CEL approach are shown in FIG.3.2 and FIG.3.3, respectively.

Johnson[JOH 83] suggested the non-dimensional parameter $\left(\rho V^{2} / Y_{d}\right)$ as a way to characterize the impact between two metallic bodies. Physically, $\rho V^{2}$ can be interpreted as the stagnation pressure of the particles flow seen as a fluid jet and $Y_{d}$ as the strength of the substrate. In a typical case in this study, that pure $\mathrm{Al}$ particle impacts on the pure $\mathrm{Al}$ 


\begin{tabular}{|c|c|c|}
\hline Properties $(\mathrm{Unit})$ & Aluminum & Copper \\
\hline Density, $\rho\left(\mathrm{kg} / \mathrm{m}^{3}\right)$ & $2.7 \times 10^{3}$ & $8.9 \times 10^{3}$ \\
Shear modulus, $\mathrm{G}(\mathrm{GPa})$ & 27 & 44.7 \\
Thermal conductivity, $\lambda(\mathrm{W} / \mathrm{m} \cdot \mathrm{K})$ & 237.2 & 386.5 \\
Specific heat, $\mathrm{c}(\mathrm{J} / \mathrm{kg} \cdot \mathrm{K})$ & 898.2 & 383 \\
Sound velocity, $\mathrm{c}_{0}(\mathrm{~m} / \mathrm{s})$ & 5386 & 3940 \\
Slope in $U_{s}$ versus $U_{p}, s$ & 1.339 & 1.489 \\
Grüneisen coefficient, $\Gamma_{0}$ & 1.97 & 2.02 \\
Yield stress, $\mathrm{A}(\mathrm{MPa})$ & 148.4 & 90 \\
Hardening constant, $\mathrm{B}(\mathrm{MPa})$ & 345.5 & 292 \\
Hardening exponent, $n$ & 0.183 & 0.31 \\
Strain rate constant, $C$ & 0.001 & 0.025 \\
Thermal softening exponent, $m$ & 0.895 & 1.09 \\
Melting temperature, $T_{m}(K)$ & 916 & 1356 \\
Reference temperature, $T_{0}(\mathrm{~K})$ & 293 & 298 \\
\hline
\end{tabular}

Table 3.2: Material properties of Aluminum and Copper used in the CEL simulation.

substrate with $700 \mathrm{~m} / \mathrm{s}$, the value of this parameter is around 8.915 , which means the hydrodynamics behaviors of particle emerge. It can be observed that the deformed shape of the particle is well reproduced by the CEL numerical approach, which is a straightforward result of the physically enhanced modeling of fluid-like particle behavior by the Eulerian numerical method. The deformation contour by using the CEL numerical approach at $840 \mathrm{~m} / \mathrm{s}$ (FIG.3.3c) does not show the concave shape as FIG.3.2c presented, this may due to the simplified initial conditions used in the simulation. For obtaining higher impact velocity, the particle must be accelerated by higher temperature gas. The side effect of this action is that both the particle and substrate obtain higher initial temperature rather than $300 \mathrm{~K}$. The particle is too small to be measured the exact impact velocity, thus either the diameters of $25 \mu \mathrm{m}$ or the impact velocity of $840 \mathrm{~m} / \mathrm{s}$ are just the mean values. Even one could measure the impact velocity, the numerical model is still idealized compared to the complexity of experimental situations. Note that in this model, the adhesion model is not included. In general, the higher the impact velocity, the larger the deformation of particle, and the deeper the crater of substrate.

\subsubsection{Evolution of the strain and temperature}

Often in the literature the researchers pay more attention to the particle or the interface than to the substrate. Actually, the substrate is also sensitive to the change of the impact velocity. The time histories of the equivalent plastic strain and temperature within the substrate during $60 n s$ after impact are shown in FIG.3.4. It can be observed that, the maximum plastic strain continues to increase after $40 \mathrm{~ns}$. This could be attributed to a change of the deformation regime from plastic to viscous flow at high impact velocities. In the present analysis, this effect is related to the third term of the Johnson-Cook strain- 


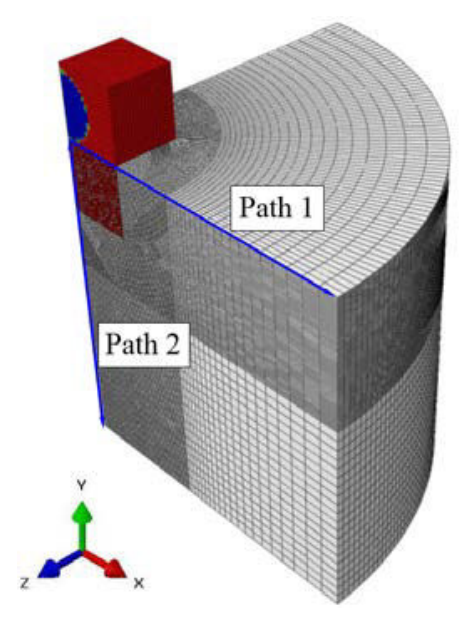

Figure 3.1: Schematic diagrams of path 1 and path 2 .

(a)

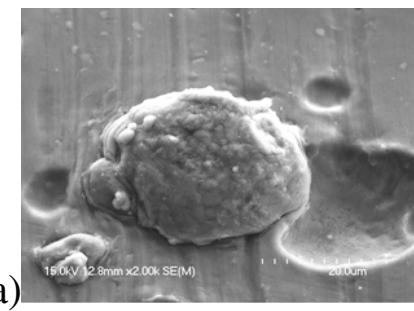

(b)

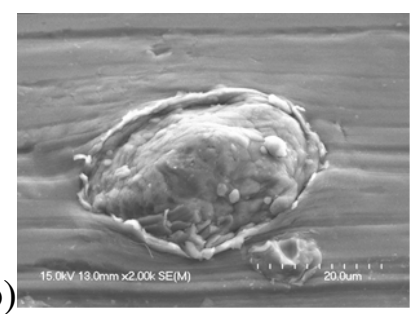

(c)

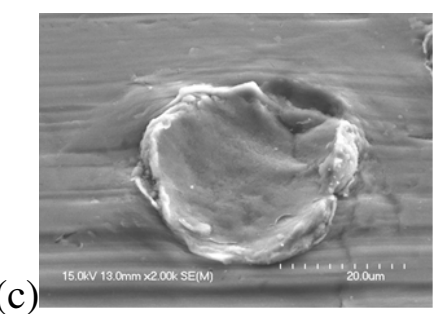

Figure 3.2: SEM images of Al/Al at impact velocity of (a) $700 \mathrm{~m} / \mathrm{s}$, (b) $780 \mathrm{~m} / \mathrm{s}$ and (c) $840 \mathrm{~m} / \mathrm{s}$.

stress law with accounts for thermal softening.

Because of the high strain rate $\left(10^{5}-10^{7} s^{-1}\right)$ occurred between $5 n s$ and $10 n s$, the temperature has a sharp increase during the same time period. After that, the substrate surface cools down with a lower cooling rate of $1.5 \times 10^{9} \mathrm{~K} / \mathrm{s}$. The general trend is, the higher the impact velocity, the higher the plastic strain rate, the higher the equivalent plastic strain and the higher the temperature of the substrate.

The interpretation of the results of modeling has so far been based on the development of field variables on selected nodes/elements in the interface. FIG.3.11 and FIG.3.12 show the equivalent plastic strain and temperature along the Path 1 on the substrate surface corresponding to various times and impact velocities, respectively. The equivalent plastic strain increases monotonically from the contact center up to a maximum value. When the equivalent plastic strain reaches the maximum value, its corresponding distance is the limit radius of contact area. The plots indicate contact area between particle and substrate grows with the progress of impact. The curve of $40 \mathrm{~ns}$ almost coincides with the curve of $60 n s$, which implies the contact area changes very little after $40 \mathrm{~ns}$. Even the contact areas keep stable after $40 \mathrm{~ns}$, the temperature does not stop decreasing, and a flattening distribution of temperature occurs as the green and dark blue curves showed in both two figures. Note that the temperature of contact center does not increase over time, it reaches 
(a)

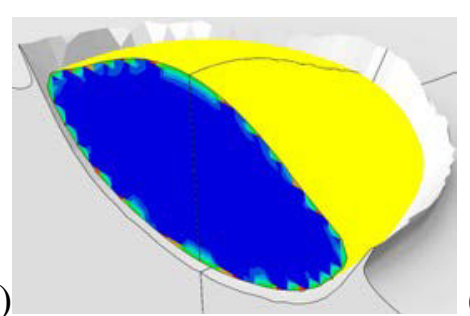

(b)

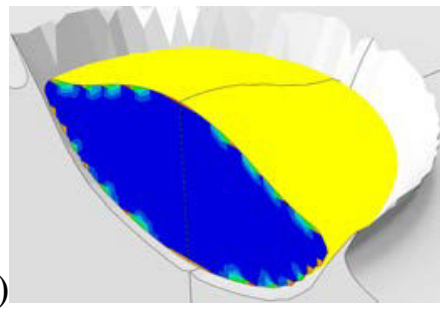

(c)

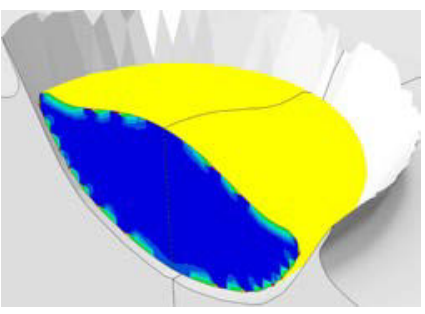

Figure 3.3: Simulation profiles of $\mathrm{Al} / \mathrm{Al}$ at impact velocity of (a) $700 \mathrm{~m} / \mathrm{s}$, (b) $780 \mathrm{~m} / \mathrm{s}$ and (c) $840 \mathrm{~m} / \mathrm{s}$.

(a)

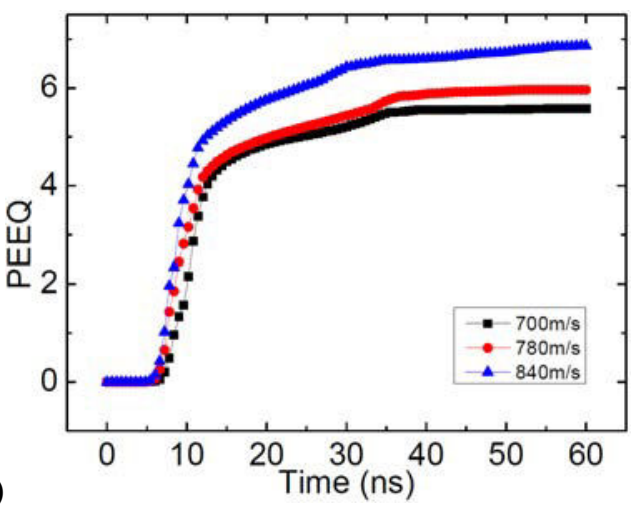

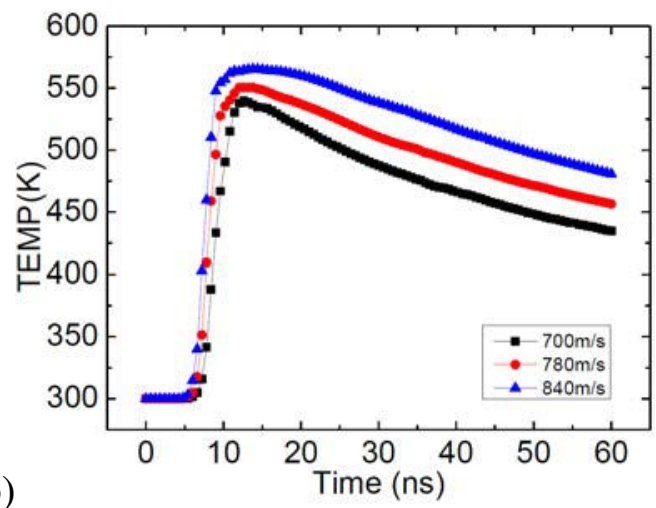

(b)

Figure 3.4: Time histories of (a) equivalent plastic strain (PEEQ) and (b) temperature (TEMP) of Al/Al by using the CEL numerical approach at $700 \mathrm{~m} / \mathrm{s}, 780 \mathrm{~m} / \mathrm{s}$ and 840 $\mathrm{m} / \mathrm{s}$.

the peak value at around $30 \mathrm{~ns}$, then it decreases about $15 \%$ for $700 \mathrm{~m} / \mathrm{s}$, and $12.7 \%$ for $840 \mathrm{~m} / \mathrm{s}$.

The corresponding calculated profiles of the equivalent plastic strain and temperature along the Path 1 and Path 2 with different impact velocities are shown in FIG.3.7 and FIG.3.8, respectively. It can be seen that the overall plastic strain and temperature distributions with different impact velocities are essentially identical. The impact velocity has a larger influence on the substrate surface, rather than the substrate depth. It is clear that greater maximum equivalent plastic strains and maximum temperature are generated during the impact at higher impact velocities. Meanwhile, the distance corresponding the maximum values moves to right along the increasing of impact velocities, which means the extent of contact area increases. Quantitatively, when the impact velocity increases from $700 \mathrm{~m} / \mathrm{s}$ to $840 \mathrm{~m} / \mathrm{s}$, the maximum equivalent plastic strain increases $12.83 \%$, and the maximum temperature increases $9.59 \%$, and the projected contact area increases $23.8 \%$. 
(a)

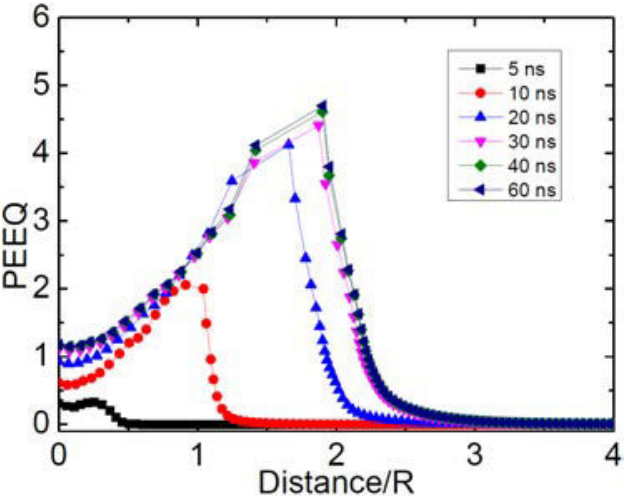

(b)

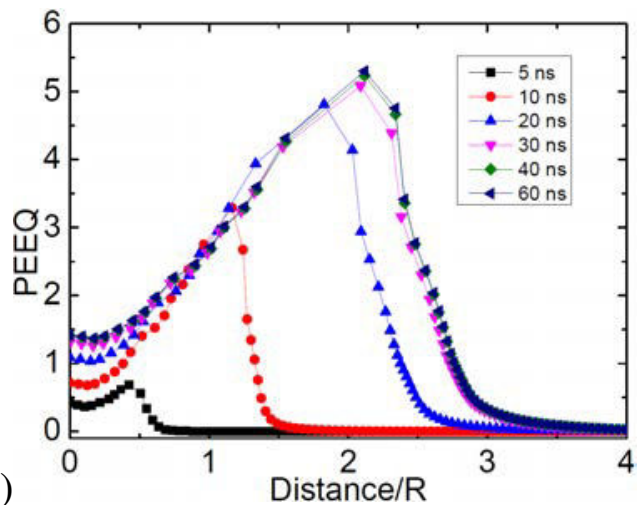

Figure 3.5: Developments of equivalent plastic strain (PEEQ) along the path 1 of $\mathrm{Al} / \mathrm{Al}$ by using the CEL numerical approach at (a) $700 \mathrm{~m} / \mathrm{s}$ and (b) $840 \mathrm{~m} / \mathrm{s}$.

(a)

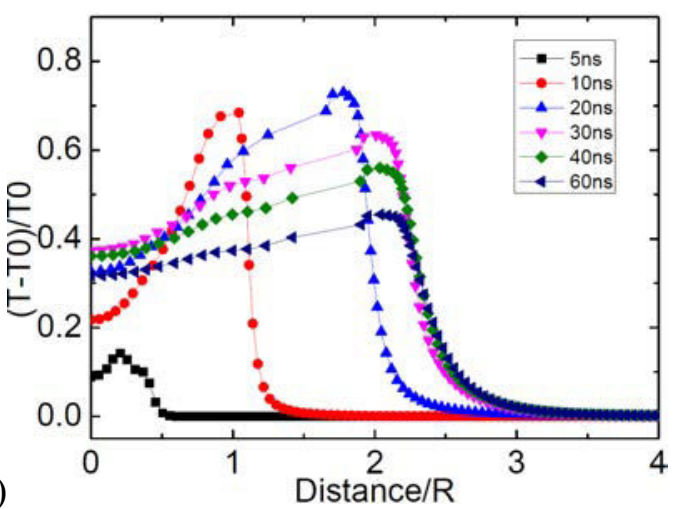

(b)

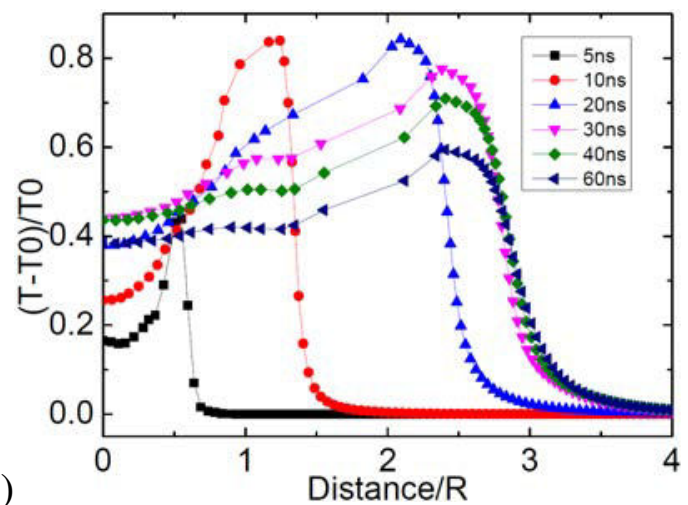

Figure 3.6: Developments of temperature (TEMP) along the path 1 of $\mathrm{Al} / \mathrm{Al}$ by using the CEL numerical approach at (a) $700 \mathrm{~m} / \mathrm{s}$ and (b) $840 \mathrm{~m} / \mathrm{s}$.

\subsection{Temperature}

\subsubsection{Deformation shape}

The deformation behaviors of $\mathrm{Cu}$ impacted particle with an initial temperature of $873 \mathrm{~K}$ obtained from experiment [KIN 10] and modeled by CEL approach are shown in FIG.3.9.

With large penetration depth, the exposed section of the crater wall is deep and nearvertical. Modeling shows that, at an impact velocity of $600 \mathrm{~m} / \mathrm{s}$, the particle expands and flattens perpendicular to the impact direction. Since the resistance of the material to shear flow collapses when the temperature tends to the melting point ( $904 K$ for Al), a better adherence or interface bonding may be expected when the initial particle temperature of $873 \mathrm{~K}$ is chosen. Unlike the $\mathrm{Al} / \mathrm{Al}$ deformation shapes, there is no gap between the particle and substrate as shown in FIG.3.9b, even without considering the adhesion model. With such high initial temperature of $873 \mathrm{~K}$, the reaction between the particle and substrate had occurred intermittently along the interface based on the KING's experiments [KIN 10]. In 
(a)

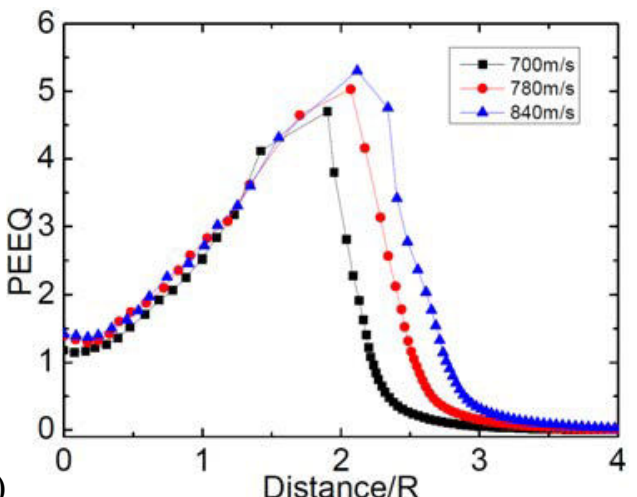

(b)

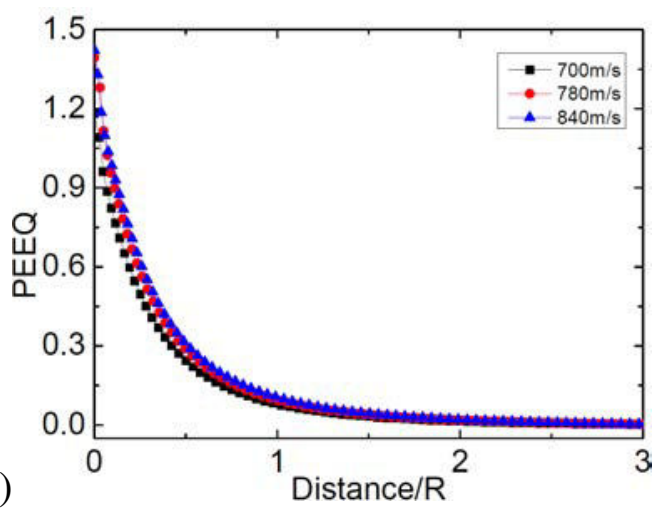

Figure 3.7: Equivalent plastic strain (PEEQ) along the (a) path 1 and (b) path 2 of Al/Al by using the CEL numerical approach at $700 \mathrm{~m} / \mathrm{s}, 780 \mathrm{~m} / \mathrm{s}$ and $840 \mathrm{~m} / \mathrm{s}$.

(a)

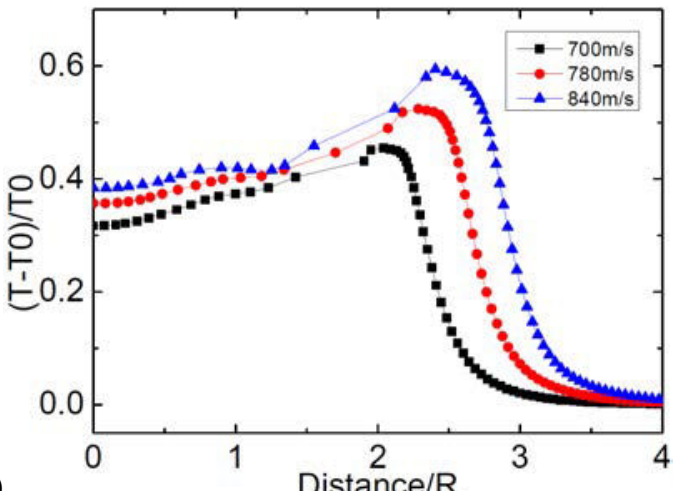

(b)

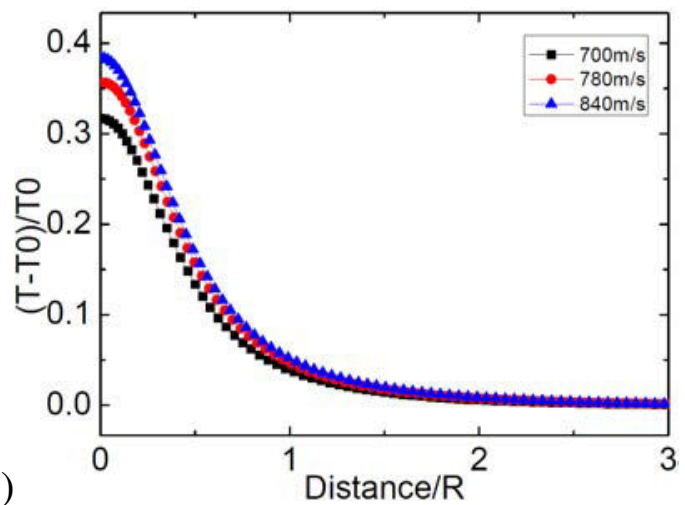

Figure 3.8: Temperature (TEMP) along the (a) path 1 and (b) path 2 of $\mathrm{Al} / \mathrm{Al}$ by using the CEL numerical approach at $700 \mathrm{~m} / \mathrm{s}, 780 \mathrm{~m} / \mathrm{s}$ and $840 \mathrm{~m} / \mathrm{s}$.

some places of interface there is an intermetallic zone up to $\backsim 50 \mathrm{~nm}$ in thickness, whose composition was close to that of stoichiometric $\mathrm{CuAl}_{2}(54.1 w t . \% \mathrm{Cu}: 45.9 w t . \% \mathrm{Al})$, which may indicate a limited amount of diffusion of $\mathrm{Cu}$ beyond the intermetallic zone into the Al. To reproduce this reaction, one needs a more accurate and sophisticated interface model. However, in the current case, due to the limit of interface model, the deformation shape of outer region of particle closing to the crater wall of substrate is slightly different from the experimental result.

\subsubsection{Evolution of the strain and temperature}

The time histories of the equivalent plastic strain and temperature within the substrate during $60 \mathrm{~ns}$ after impact are shown in FIG.3.10. The rise of the plastic strain after initial contact of $15 \mathrm{~ns}$ can be attributed to the thermal softening. Near the condition for thermal softening, the resistance of material to shear flow is generally low, which means the material could undergo excessive deformation for a few amount of imposed shear 

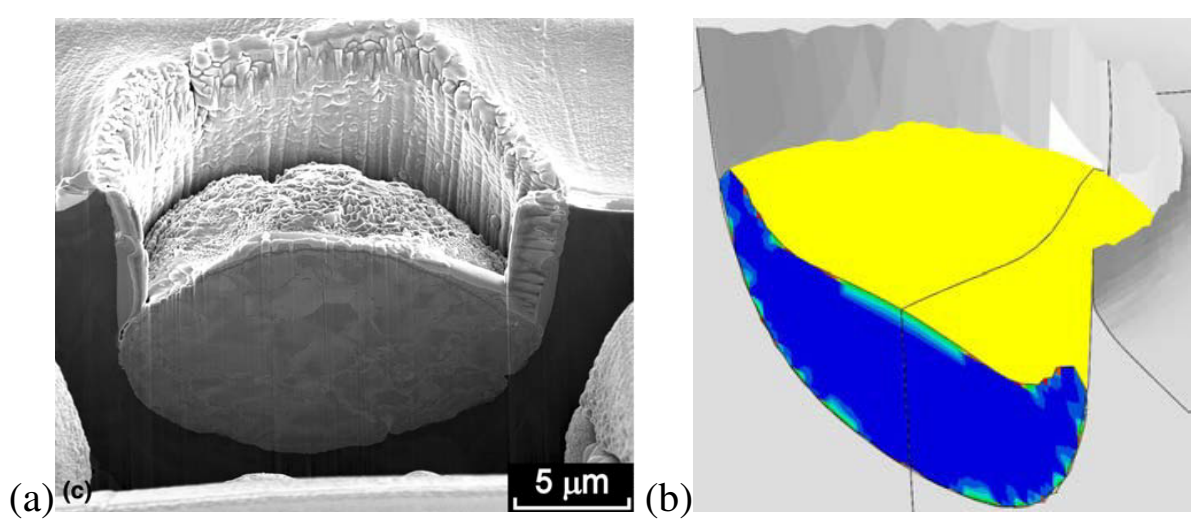

Figure 3.9: (a) SEM image and (b) simulation profile of $\mathrm{Cu} / \mathrm{Al}$ with an initial particle temperature of $873 \mathrm{~K}$.

stress by approaching the melting temperature. That's why a higher initial temperature leads to a higher equivalent plastic strain (blue curve in the FIG.3.11a). Even the initial particle temperature increases $200 \mathrm{~K}$, the substrate does not increase $200 \mathrm{~K}$. On the contrary, it only increases $\backsim 5 K$, which is the straightforward results of adiabatic heating.

(a)

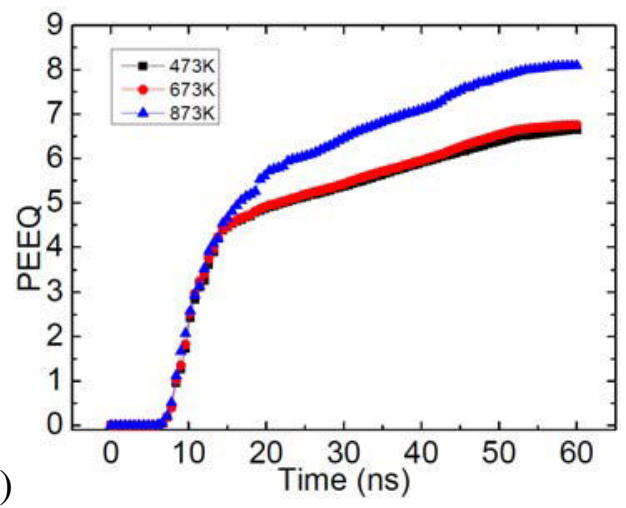

(b)

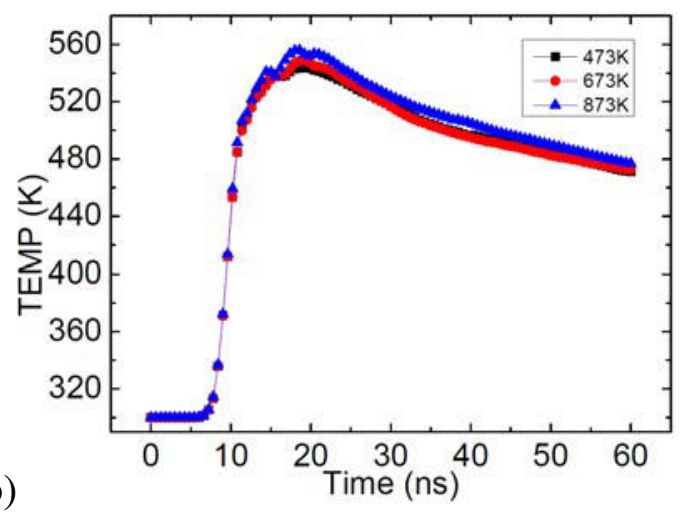

Figure 3.10: Time histories of (a) equivalent plastic strain (PEEQ) and (b) temperature (TEMP) of $\mathrm{Cu} / \mathrm{Al}$ by using the CEL numerical approach at the initial particle temperatures of $473 K, 673 K$ and $873 K$.

FIG.3.11 and FIG.3.12 show the equivalent plastic strain and temperature along the Path 1 on the substrate surface corresponding to various times, respectively. The maximum equivalent plastic strain continues to rise during the whole calculation time of 60 $n s$, and the difference of equivalent plastic strain between two observation moments becomes smaller and smaller. After a short time of initial contact, the temperature increases about $80 \%$, and the higher temperature is situated in very local area. The temperature of contact center increase monotonically, but the temperature near the edge of contact area decreases slowly after reaching the peak value. At $60 n s$, the temperature distribution along the substrate crater is roughly uniform.

The corresponding calculated profiles of the equivalent plastic strain and tempera- 
(a)

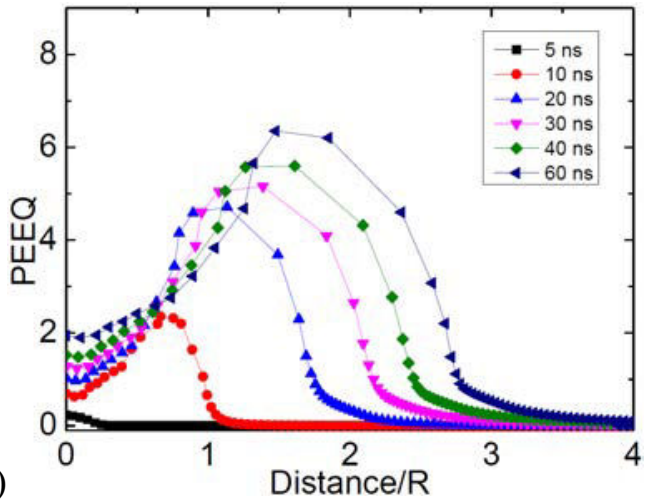

(b)

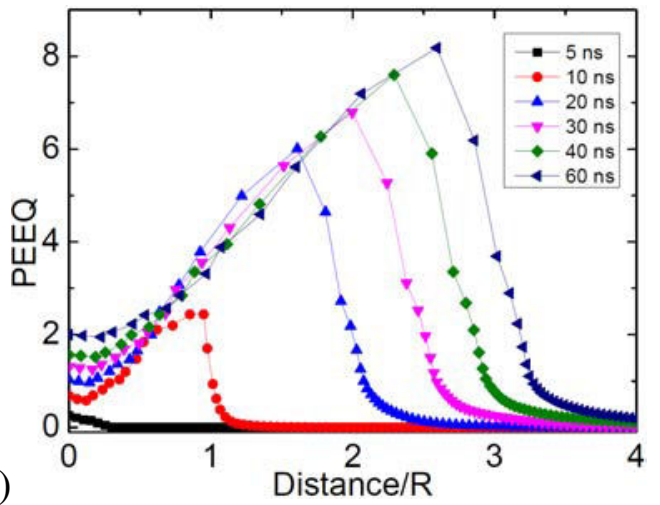

Figure 3.11: Developments of equivalent plastic strain (PEEQ) along the path 1 of $\mathrm{Cu} / \mathrm{Al}$ by using the CEL numerocal approach at the initial particle temperatures of (a) $473 K$ and (b) $873 \mathrm{~K}$.

(a)

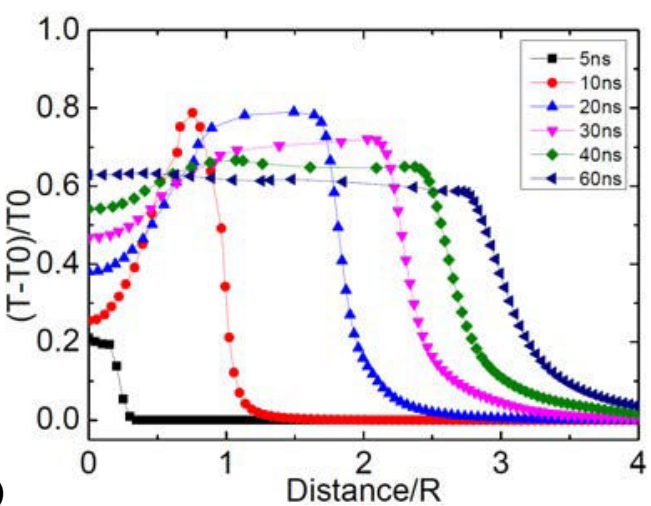

(b)

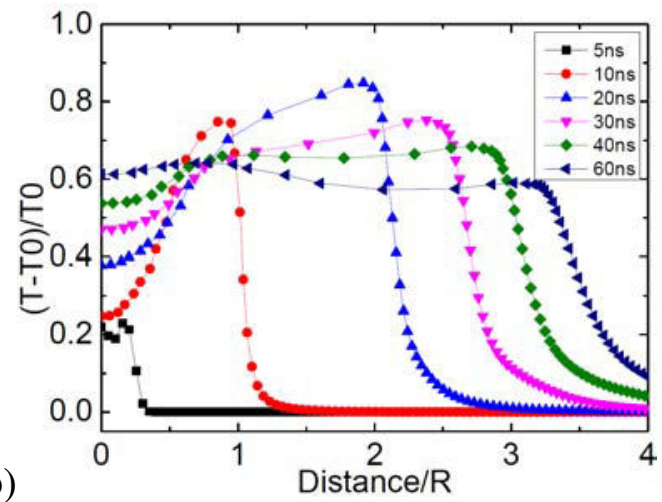

Figure 3.12: Developments of temperature (TEMP) along the path 1 of $\mathrm{Cu} / \mathrm{Al}$ by using the CEL numerical approach at the initial particle temperatures of (a) $473 K$ and (b) 873 K.

ture along the Path 1 and Path 2 with different initial particle temperatures are shown in FIG.3.13 and FIG.3.14, respectively. The temperature has a larger influence on the substrate surface where directly contacts with particle, that is probably due to the fact that in the present simulations only the temperature of the particle is changed and the substrate temperature remain $300 \mathrm{~K}$. The evolution along the depth of substrate change very few no matter which initial temperature of particle. When the initial particle temperature increases from $473 \mathrm{~K}$ to $673 \mathrm{~K}$, the maximum plastic strain and temperature within the substrate surface increase $11.53 \%$ and $0.19 \%$, respectively; when the initial particle temperature increase from $673 \mathrm{~K}$ to $873 \mathrm{~K}$, however, the plastic strain and temperature increase $30.99 \%$ and $0.37 \%$, respectively. The higher the temperature, the more significant the influence of temperature.

Commercial cold spray systems heat the gas to temperature up to 800-1100 $\mathrm{K}$ depending on the technology used. Some researchers have suggested that local particle melting must occur at the particle/substrate interface due to the high impact energy in a 
process similar to explosion welding [LI 10a, GUE 09]. Conversely, others have reported no melting at the interface and have attributed adhesion uniquely to the intense plastic deformation under a very high-localized pressure and related phenomena at the interfaces [GRU 03, LEE 04]. For Cu particle with an initial temperature of $873 \mathrm{~K}$, the maximum temperature is $964 K$, which is well below the melting temperature of $1356 K$. Interfacial melting does not happen for the particle with an initial temperature of $473 \mathrm{~K}$ and $673 \mathrm{~K}$ as well, which the temperature is always below the melting temperature during the whole process of calculation. Moreover, the temperature of Al substrate is below the melting temperature of $904 \mathrm{~K}$ for all the situations. The temperature at the contact zone caused by the adiabatic shear instability can achieve a value higher than the melting point of spray materials, promoting the formation of a thin melted layer. However, for the $\mathrm{Cu} / \mathrm{Al}$ cases studied in this section, no such thin melted layer or localized region is found.

The fundamental models of heat transfer are Advection, Conduction/Diffusion, Convection and Radiation. We have not considered the influence of carrier gas and the interaction between the gas and particles, as well as the interaction between gas and substrate. Thus, thermal conduction and thermal radiation are two dominant heat transfer forms in our study. For clarifying, there is no thermal conductance between particle and the substrate; instead, the heat of particle generated by the plastic deformation is only conducted inside itself, and the same as the substrate. All matter with a temperature greater than absolute zero emits thermal radiation. The thermal radiation is more significant when the temperature of material is higher. Taking the thermal radiation into account will definitely bring the more reasonable results, especially for the coupled thermal-stress analysis, and that's the work we are eager to do in the next step.

(a)

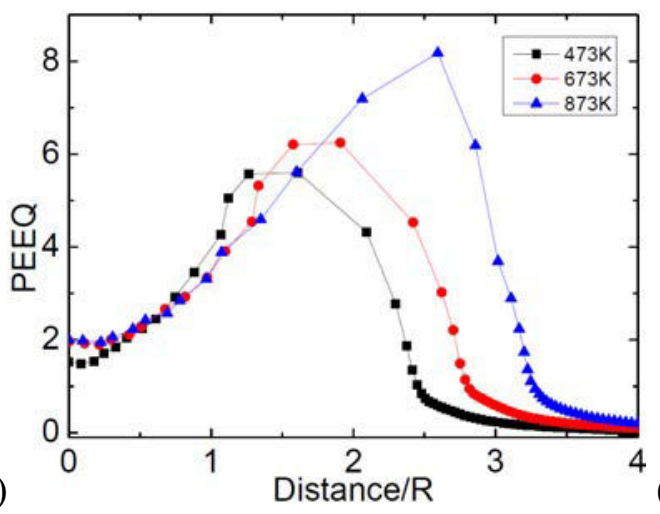

(b)

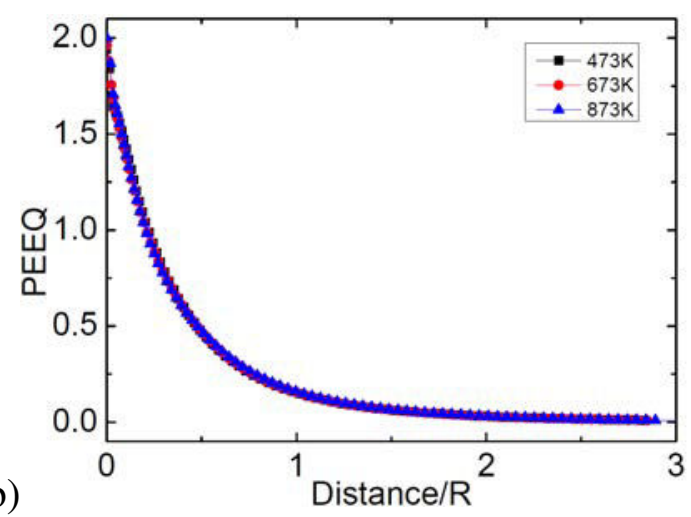

Figure 3.13: Equivalent plastic strain (PEEQ) along the (a) path 1 and (b) path 2 of $\mathrm{Cu} / \mathrm{Al}$ by using the CEL numerical approach at the initial particle temperatures of $473 \mathrm{~K}, 673 \mathrm{~K}$ and $873 \mathrm{~K}$. 

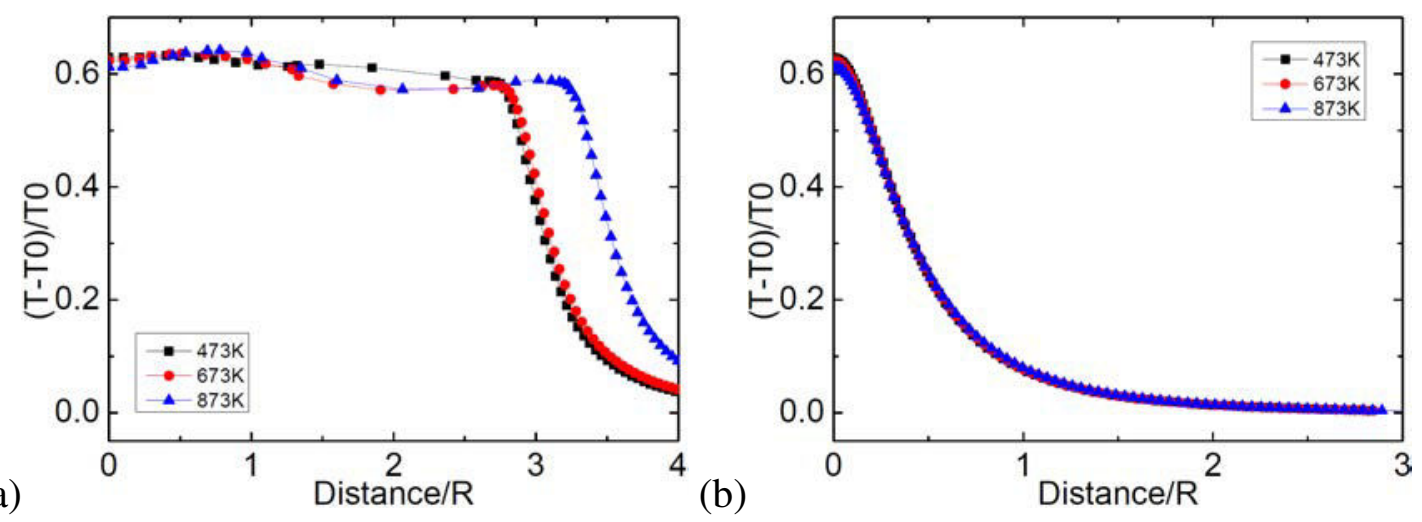

Figure 3.14: Temperature (TEMP) along the (a) path 1 and (b) path 2 of $\mathrm{Cu} / \mathrm{Al}$ by using the CEL numerical approach at the initial particle temperatures of $473 \mathrm{~K}, 673 \mathrm{~K}$ and 873 $K$.

\subsection{Friction coefficient}

\subsubsection{Deformation shape}

The deformation behaviors of $\mathrm{Cu}$ impacted particle at different friction coefficient obtained from literature [LI 09a] and modeled by CEL approach are shown in FIG.3.15 and FIG.3.16, respectively. Since there are no usable experimental results concerning the effect of friction coefficient on the deposition behaviors of cold spray particles, the simulation results published in the literature are chosen. Basically, the friction coefficient has a less significant influence on the deformation shape of $\mathrm{Cu}$ particle and $\mathrm{Cu}$ substrate.

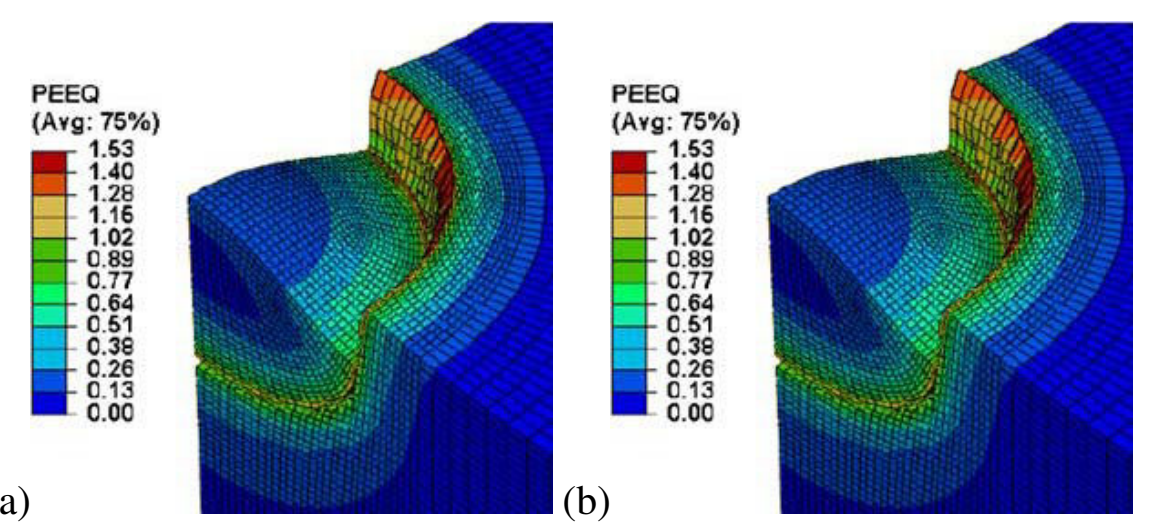

Figure 3.15: Simulation profiles of $\mathrm{Cu} / \mathrm{Cu}$ with friction coefficient of (a) 0.2 and (b) 0.5 by Li. et al [LI 09a] using the Lagrangian numerical approach. 
(a)

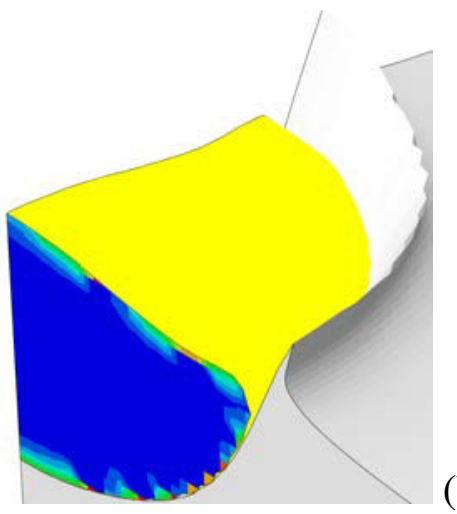

(b)

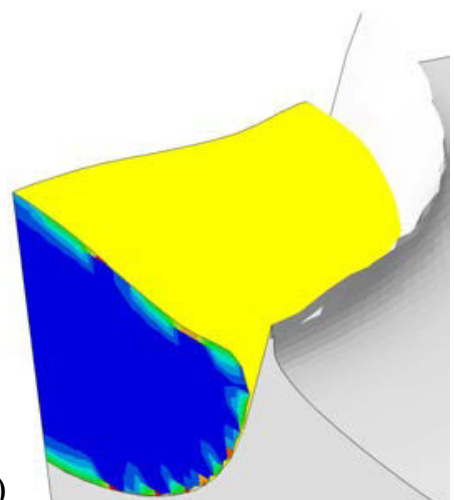

Figure 3.16: Simulation profiles of $\mathrm{Cu} / \mathrm{Cu}$ with friction coefficients of (a) 0.2 and (b) 0.5 by using the CEL numerical approach.

\subsubsection{Evolution of the strain and temperature}

The time histories of the equivalent plastic strain and temperature within the substrate during $60 n s$ after impact are shown in FIG.3.17. The equivalent plastic strain increases faster with a strain rate of $6 \times 10^{6} s^{-1}$ between $2 n s$ and $10 n s$, and then increases slowly with a strain rate of $1 \times 10^{5} \mathrm{~s}^{-1}$ till the end of calculation. The higher the friction coefficient, the higher the equivalent plastic strain of the substrate surface; however, the friction energy contributes very little to the rise of temperate of substrate. When the friction coefficient increases from 0.2 to 0.5 , the maximum temperature increase only slightly $0.96 \%$, and the friction energy divided by the internal energy is less than $8 \%$ for all the cases as displays in the FIG.3.18. The friction has a more significant influence at the initial contact, and after $35 n s$ it only accounts for less than $1 \%$ of internal energy. The friction energy decreases along with the rise of friction coefficient. Increasing friction coefficient leads to a sharp decrease of sliding distance, which results in the reducing of friction energy.

(a)

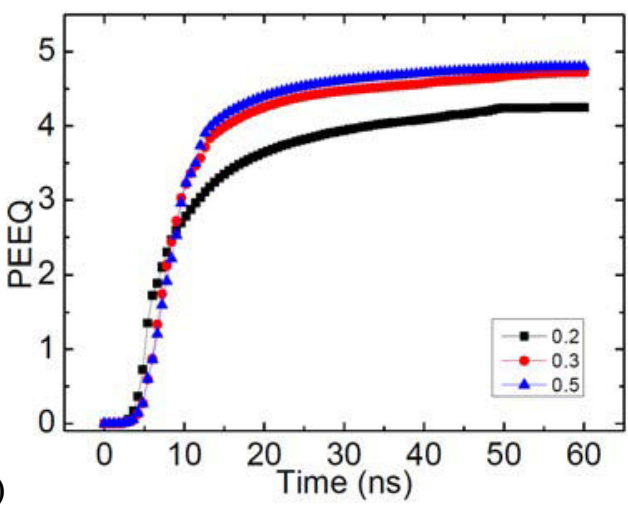

(b)

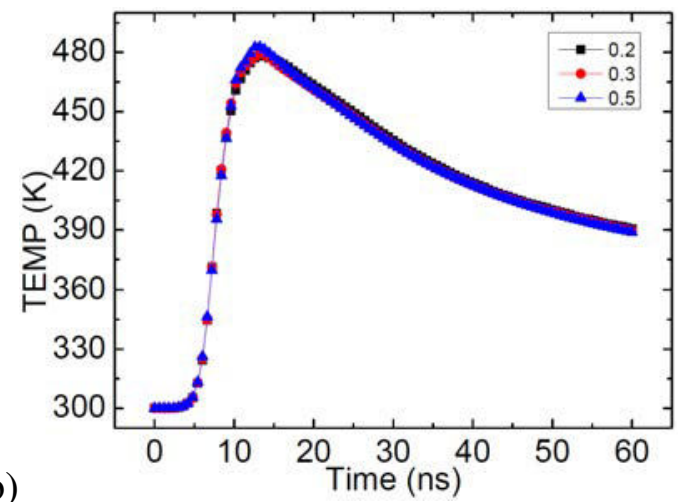

Figure 3.17: Time histories of (a) equivalent plastic strain (PEEQ) and (b) temperature (TEMP) of $\mathrm{Cu} / \mathrm{Cu}$ by using the CEL numerical approach with friction coefficients of 0.2 , 0.3 and 0.5 . 


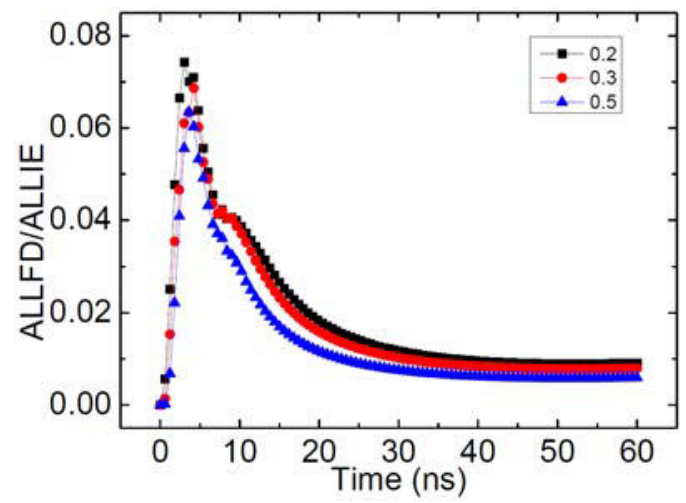

Figure 3.18: Time histories of friction energy (ALLFD) divided by internal energy (ALLIE) of $\mathrm{Cu} / \mathrm{Cu}$ by using the CEL numerical approach with friction coefficients of $0.2,0.3$ and 0.5 .

FIG.3.19 and FIG.3.20 show the equivalent plastic strain and temperature along the Path 1 on the substrate surface corresponding to various times and friction coefficients, respectively. The corresponding calculated profiles of the equivalent plastic strain and temperature along the Path 1 and Path 2 with different friction coefficient are shown in FIG.3.21 and FIG.3.22, respectively. Comparing to the other two parameters discussed in the previous sections, the effect of friction coefficient is relatively small. Therefore, in the following chapter, a small friction coefficient of 0.3 is used in modeling multiple particle impact because it may be appropriate in practice with a little friction.

(a)

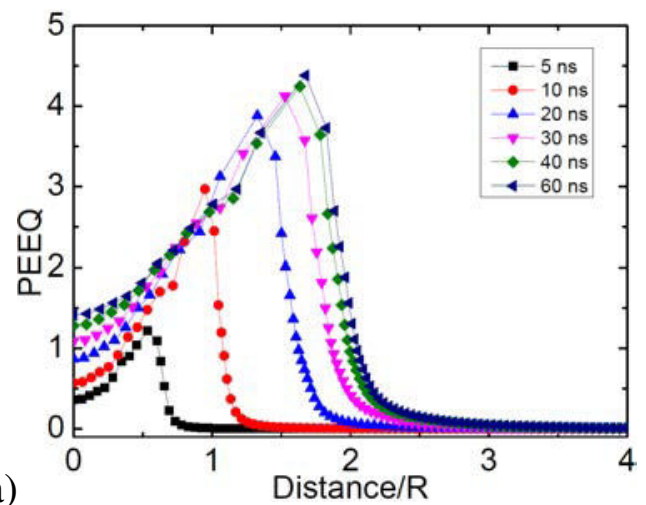

(b)

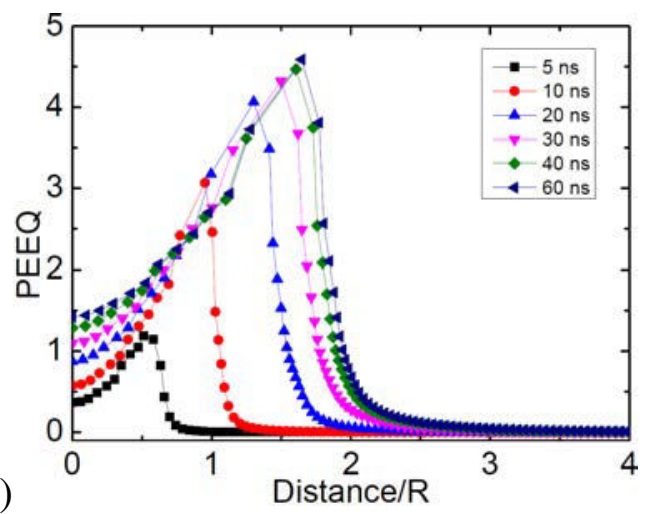

Figure 3.19: Developments of equivalent plastic strain (PEEQ) along the path 1 of $\mathrm{Cu} / \mathrm{Cu}$ by using the CEL numerical approach with friction coefficients of (a) 0.2 and (b) 0.5 . 

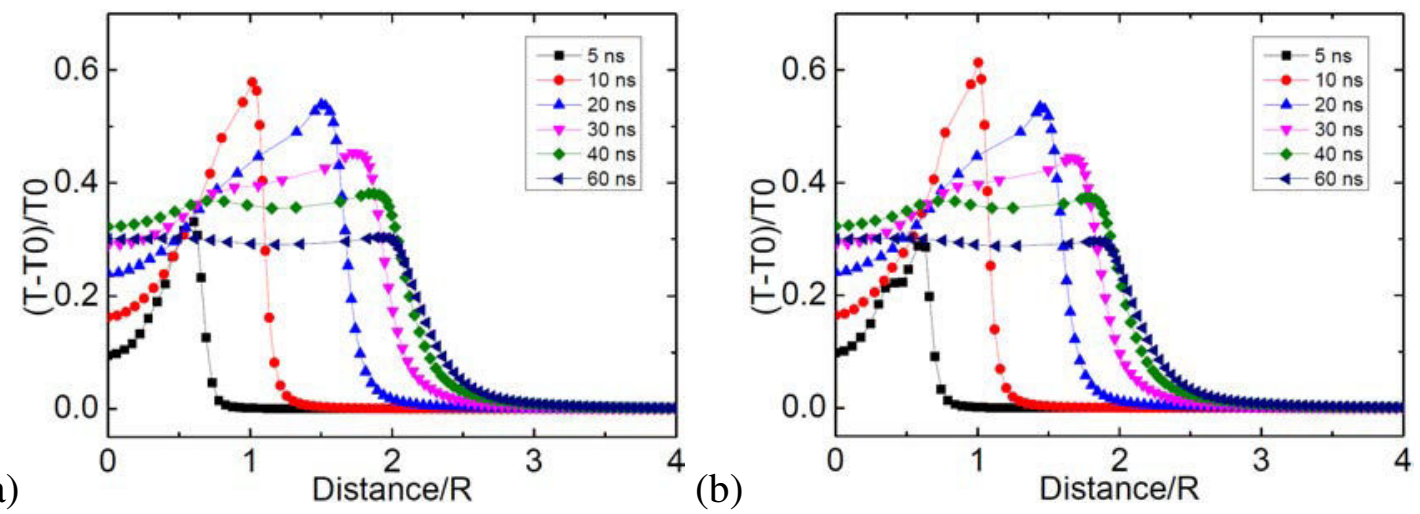

Figure 3.20: Developments of temperature (TEMP) along the path 1 of $\mathrm{Cu} / \mathrm{Cu}$ by using the CEL numerical approach with friction coefficients of (a) 0.2 and (b) 0.5 .

(a)

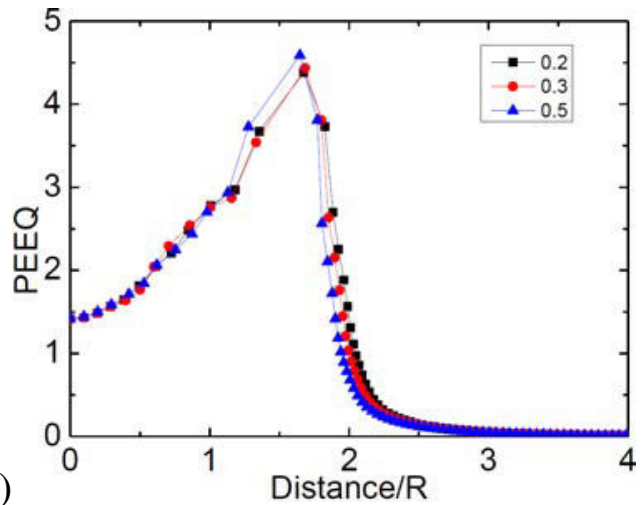

(b)

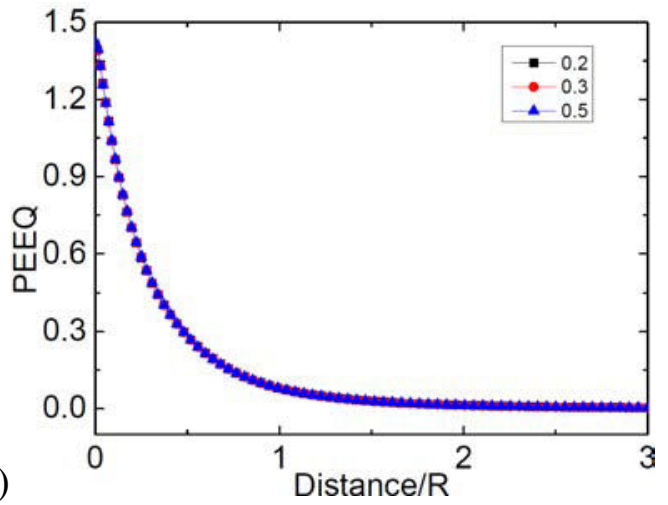

Figure 3.21: Equivalent plastic strain (PEEQ) along the (a) path 1 and (b) path 2 of $\mathrm{Cu} / \mathrm{Cu}$ by using the CEL numerical approach with friction coefficients of $0.2,0.3$ and 0.5 .

\subsection{Material}

\subsubsection{Deformation shape}

Material of $\mathrm{Cu}$ and $\mathrm{Al}$ could be classified into four impact cases, according to their physical and mechanical properties. The Vickers hardness of $\mathrm{Cu}$ is $369 \mathrm{MPa}$, and 167 $M P a$ for Al. Therefore, these four impact cases are Al/Al (soft/soft), Cu/Al (hard/soft), $\mathrm{Cu} / \mathrm{Cu}$ (hard/hard) and $\mathrm{Al} / \mathrm{Cu}$ (soft/hard). The deformation shapes of the former three cases have been showed in the above sections, here in this section, only the deformation behaviors of Al impacted particle at impact velocity of $500 \mathrm{~m} / \mathrm{s}$ obtained from experiment [KIN 08] and modeled by CEL approach are shown in FIG.3.23. The case of $\mathrm{Al} / \mathrm{Cu}$, which is dissimilar combination, reveal quite different deformation behaviors as compared to the previously mentioned similar cases $(\mathrm{Al} / \mathrm{Al}$ and $\mathrm{Cu} / \mathrm{Cu})$. The initial kinetic energy of the particle is mostly dissipated into plastic deformation of the relatively soft counterpart. FIG.3.23b shows a flattened particle with a very slightly deformed substrate which agrees well with the KING's experiment results. It is worth to recall that in the case 

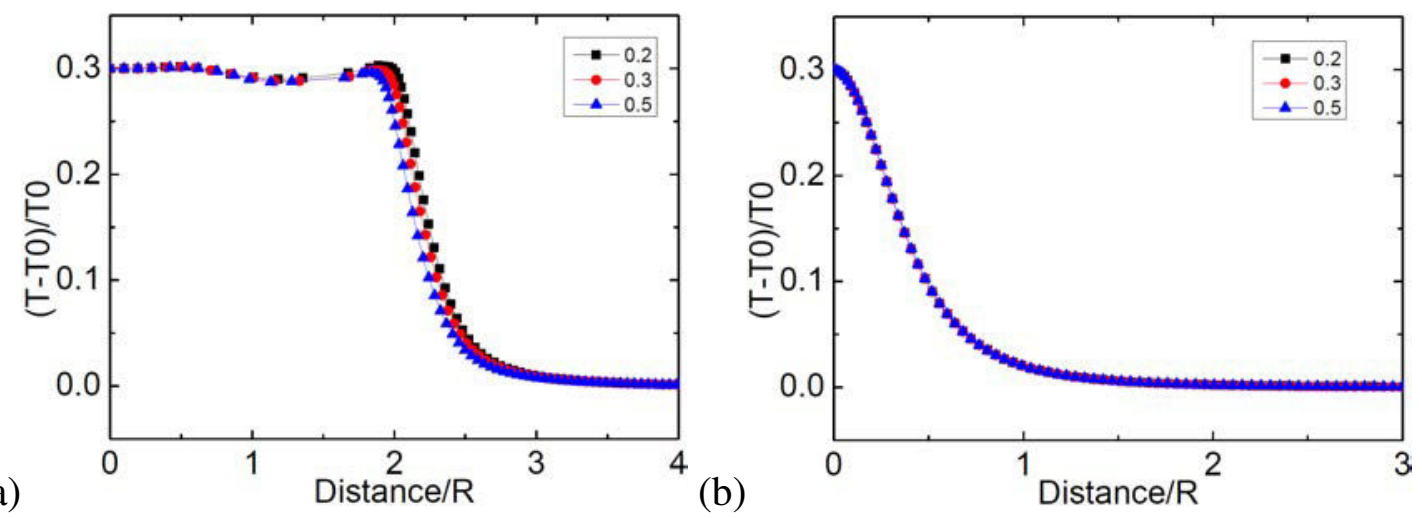

Figure 3.22: Temperature (TEMP) along the (a) path 1 and (b) path 2 of $\mathrm{Cu} / \mathrm{Cu}$ by using the CEL numerical approach with friction coefficients of 0.2, 0.3 and 0.5.

of $\mathrm{Al} / \mathrm{Al}$ (FIG.3.3), relatively large deformation is observed as compared to the $\mathrm{Cu} / \mathrm{Cu}$ case (FIG.3.16). This can be attributed mainly to the relatively low density and high heat capacity of $\mathrm{Al}$ as compared to $\mathrm{Cu}$. The simulation result of $\mathrm{Cu} / \mathrm{Al}$ (FIG.3.9b) demonstrates a deeply penetrated substrate with a less deformed particle. Taking into account the different physical and mechanical properties between particle and the substrate, the results of critical velocity are understandable, which the soft substrate needs less kinetic energy to deform than hard substrate.
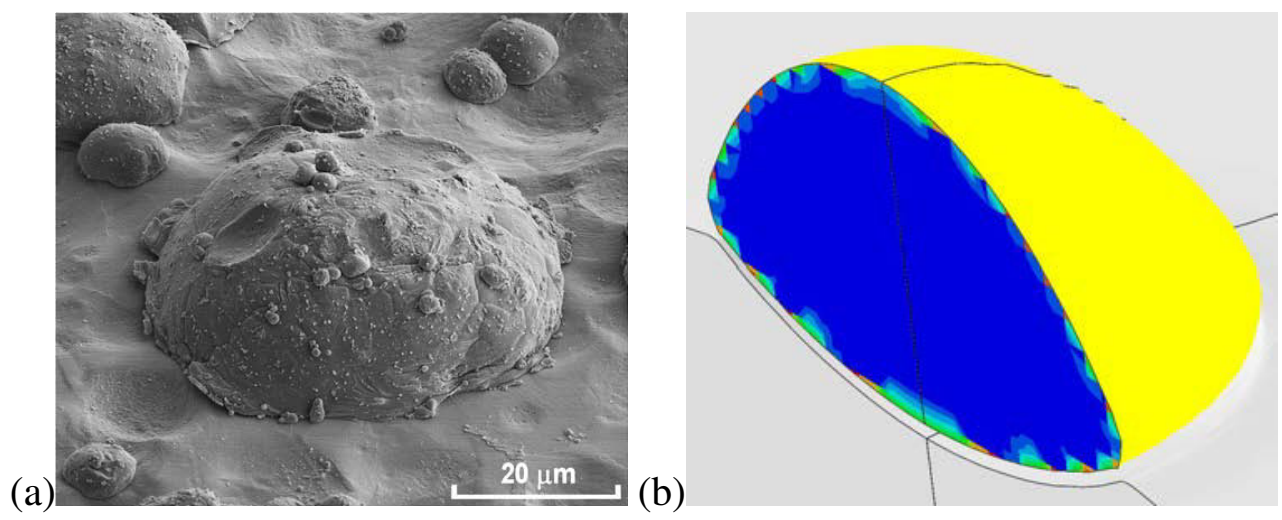

Figure 3.23: (a) SEM image and (b) simulation profile of $\mathrm{Al} / \mathrm{Cu}$ at impact velocity of 500 $\mathrm{m} / \mathrm{s}$.

\subsubsection{Evolution of the strain and temperature}

The time histories of the equivalent plastic strain and temperature within the substrate during $60 n s$ after impact are shown in FIG.3.24. The density of $\mathrm{Cu}$ is higher than that of $\mathrm{Al}$, thus the initial kinetic energy of $\mathrm{Cu}$ particle is higher with the same radius and same impact velocity, and the $\mathrm{Cu}$ particle undergoes significantly larger amount of plastic deformation (FIG.3.24a), and much higher temperature is achieved accordingly (FIG.3.24b). 
The material of particle predominantly affects the deposition process and deformation than that of the substrate. The $\mathrm{Cu}$ particle impact cases $(\mathrm{Cu} / \mathrm{Cu}$ and $\mathrm{Cu} / \mathrm{Al})$ have similar development of plastic strain even their substrate material are dissimilar.

(a)

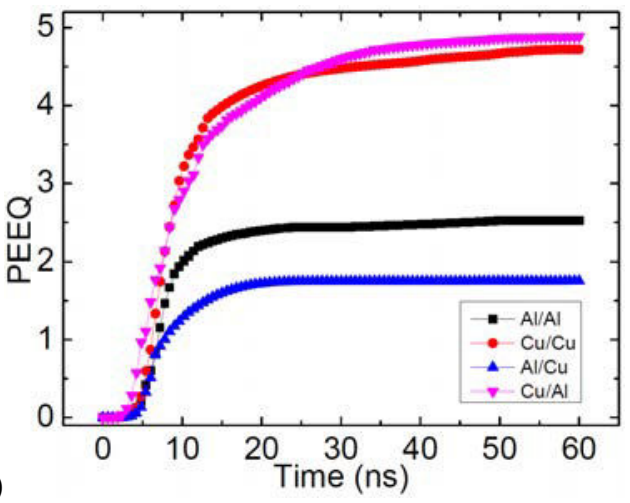

(b)

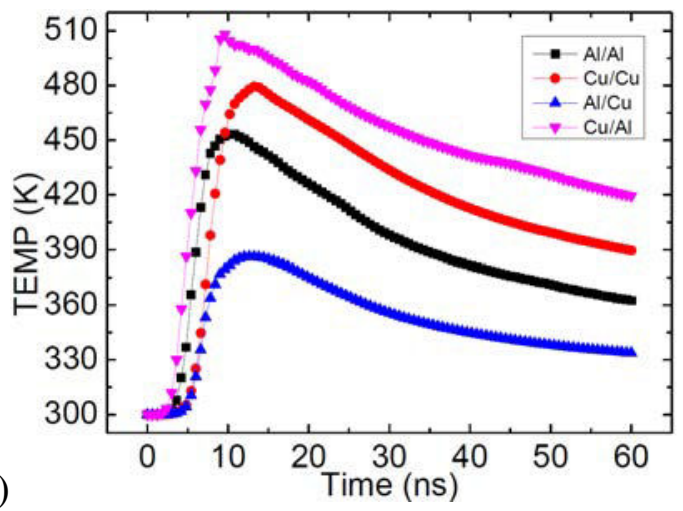

Figure 3.24: Time histories of (a) equivalent plastic strain (PEEQ) and (b) temperature (TEMP) of $\mathrm{Al} / \mathrm{Al}, \mathrm{Cu} / \mathrm{Cu}, \mathrm{Al} / \mathrm{Cu}$ and $\mathrm{Cu} / \mathrm{Al}$ by using the $\mathrm{CEL}$ numerical approach at 500 $m / s$.

FIG.3.25a and FIG.3.25b show the equivalent plastic strain and temperature along the Path 1 on the substrate surface corresponding to various times, respectively. Because these results of $\mathrm{Al} / \mathrm{Al}, \mathrm{Cu} / \mathrm{Cu}$ and $\mathrm{Cu} / \mathrm{Al}$ have already showed before, here only the results of $\mathrm{Al} / \mathrm{Cu}$ are given. Not only the plastic deformation of substrate of $\mathrm{Al} / \mathrm{Cu}$ is significantly lower than the other three ones, but also the contact time is shorter. The contact time is $25 n s$ for $\mathrm{Al} / \mathrm{Al}, 27 n s$ for $\mathrm{Al} / \mathrm{Cu}, 47 n s$ for $\mathrm{Cu} / \mathrm{Al}$ and $53 n s$ for $\mathrm{Cu} / \mathrm{Cu}$ indicated in FIG.3.26(a). Critical velocities for $\mathrm{Al} / \mathrm{Al}$ and $\mathrm{Al} / \mathrm{Cu}$ are estimated as $766 \mathrm{~m} / \mathrm{s}$ and 634 $\mathrm{m} / \mathrm{s}$ [GRU 04], respectively. The impact velocity of $500 \mathrm{~m} / \mathrm{s}$ is below its critical velocity, the $\mathrm{Al}$ particle rebounds after a short contact time. Critical velocities for $\mathrm{Cu} / \mathrm{Al}$ and $\mathrm{Cu} / \mathrm{Cu}$ are estimated as $507 \mathrm{~m} / \mathrm{s}$ and $571 \mathrm{~m} / \mathrm{s}$ [GRU 04], respectively. Closing to the critical velocity, the $\mathrm{Cu}$ particle has a sufficient time to contact and deform. As shown in FIG.3.26(b), the developments of kinetic energy after rebounding are not monotonically, actually they damp periodically, which implies the vibration of particle may eventually stop if the calculation time is long enough or due to the second impacts of following particles.

The corresponding calculated profiles of the equivalent plastic strain and temperature along the Path 1 and Path 2 with different material combinations are shown in FIG.3.27 and FIG.3.28, respectively. Due to the high kinetic energy and sufficient contact time, the projected contact area of $\mathrm{Cu}$ particles $(\mathrm{Cu} / \mathrm{Cu}$ and $\mathrm{Cu} / \mathrm{Al})$ is almost 4 times as that of $\mathrm{Al}$ particles. Besides, the equivalent plastic strains tend to zero when the radius of substrate exceeds $4 \mathrm{R}$ and the depth of substrate exceeds $2 \mathrm{R}$. The temperature distributions are also limited within this cylinder with a radius of $4 \mathrm{R}$ and a height of $2 \mathrm{R}$. Therefore, the dimensions of this model are enough to investigate the single impact problem following the principles of accuracy and efficiency. 
(a)

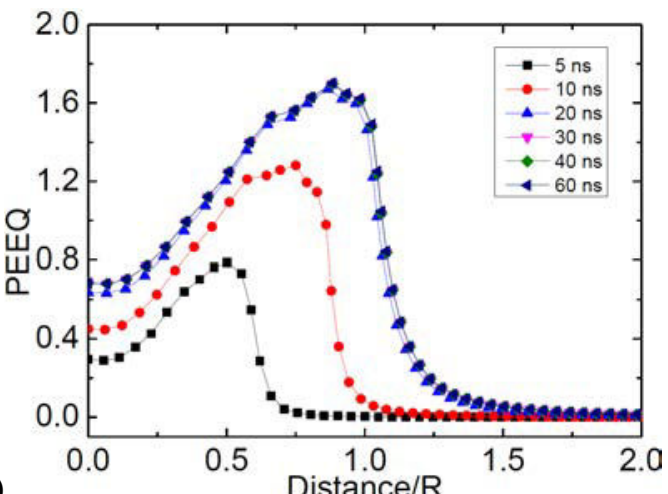

(b)

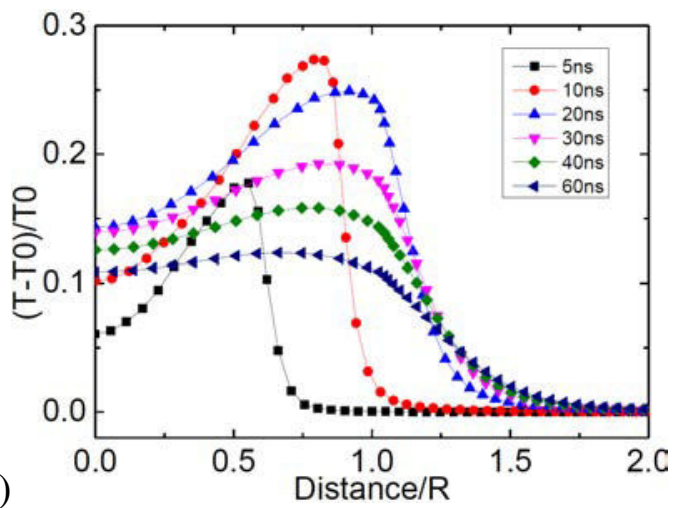

Figure 3.25: Developments of (a) equivalent plastic strain (PEEQ) and (b) temperature (TEMP) along the path 1 of $\mathrm{Al} / \mathrm{Cu}$ by using the CEL numerical approach at $500 \mathrm{~m} / \mathrm{s}$.

(a)

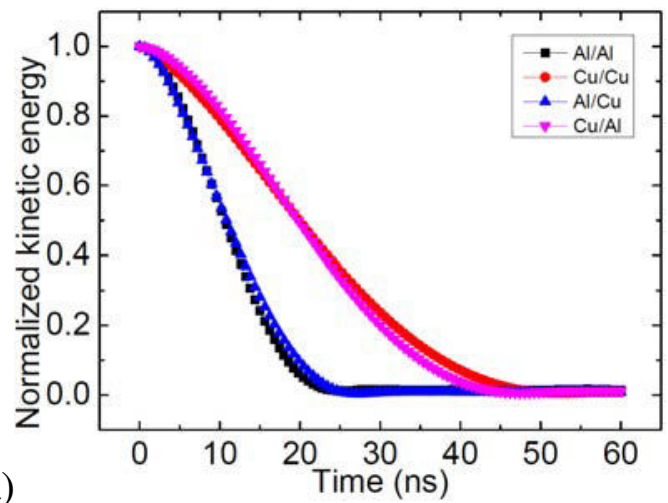

(b)

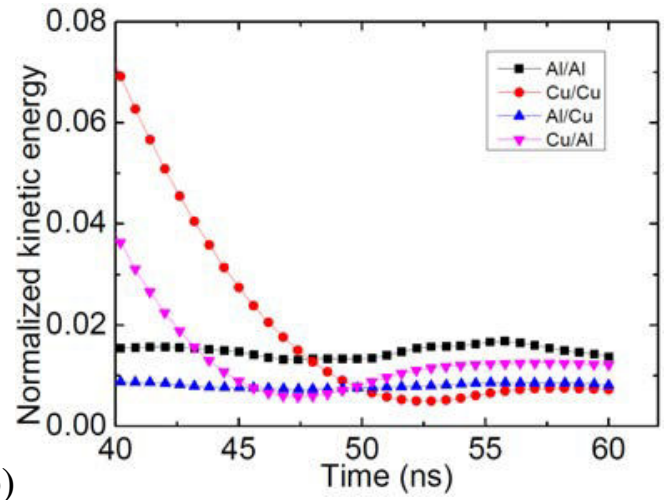

Figure 3.26: Time histories of normalized kinetic energy by using the CEL numerical approach at $500 \mathrm{~m} / \mathrm{s}$. (a) $0-60 \mathrm{~ns}$; (b) $40-60 \mathrm{~ns}$.

\subsection{Discussion}

\subsubsection{Energy dissipation and restitution coefficient}

In most problems, the mechanical engineer seeks to answer this fundamental question: What is the relationship between the velocities before and after impact? The restitution coefficient, which is usually used to characterize the change in kinetic energy during the impact, is one of the important parameters needed to predict post-impact motion of the particle. There are several definitions for the restitution, which were proposed by NEWTON, POISSON and STRONGE [WU 01].

- The restitution coefficient $e$ relates the relative velocities before and after impact was first introduced by Newton[FAI 00]:

$$
v_{1 r}-v_{2 r}=-e\left(v_{1 i}-v_{2 i}\right)
$$

where the subscripts 1 and 2 refer to the particle and substrate separately and $i$ and 
(a)

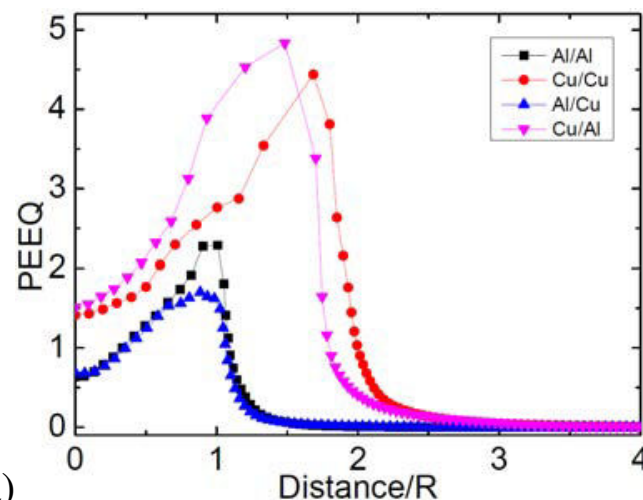

(b)

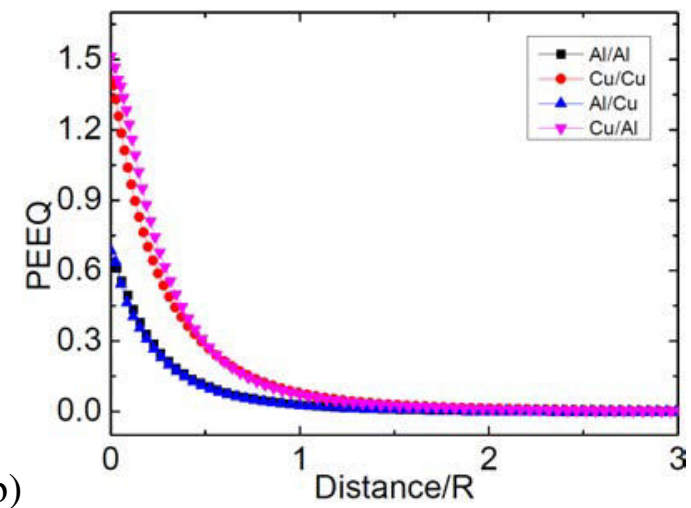

Figure 3.27: Equivalent plastic strain (PEEQ) along the (a) path 1 and (b) path 2 of $\mathrm{Al} / \mathrm{Al}$, $\mathrm{Cu} / \mathrm{Cu}, \mathrm{Al} / \mathrm{Cu}$ and $\mathrm{Cu} / \mathrm{Al}$ by using the CEL numerical approach at $500 \mathrm{~m} / \mathrm{s}$.

(a)

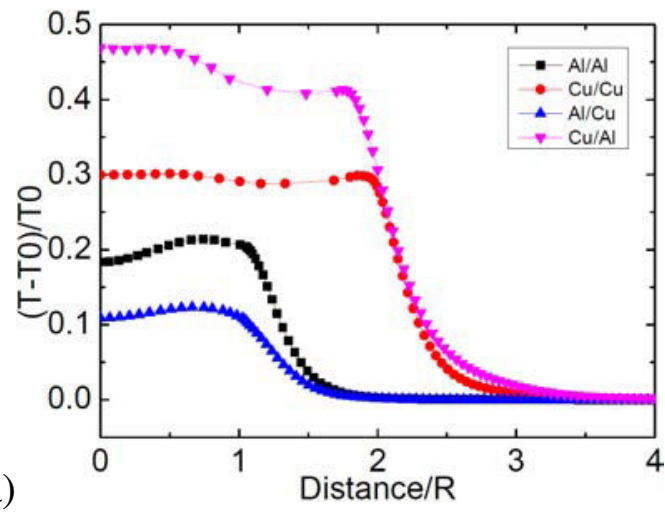

(b)

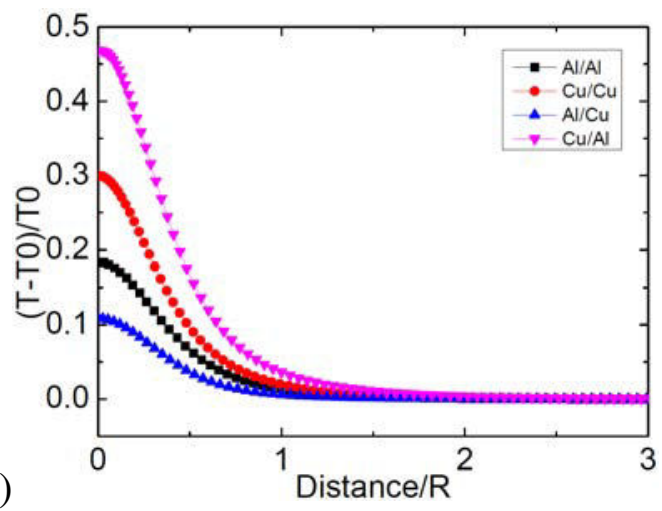

Figure 3.28: Temperature (TEMP) along the (a) path 1 and (b) path 2 of $\mathrm{Al} / \mathrm{Al}, \mathrm{Cu} / \mathrm{Cu}$, $\mathrm{Al} / \mathrm{Cu}$ and $\mathrm{Cu} / \mathrm{Al}$ by using the CEL numerical approach at $500 \mathrm{~m} / \mathrm{s}$.

$r$ stand for initial and rebound states of impact.

- POISSON defined the restitution coefficient as the ratio of the impulse during the restitution phase to the impulse during the compression phase of the impact.

- In STRONGE's definition, the restitution coefficient is expressed as the ratio of work done by the normal force during the restitution to that during the compression.

However, it can be seen from FIG.3.29 that the rebound velocity varies from node to node and from time to time due to the influence of stress wave propagation. Note that the particle has rebounded since $24.6 \mathrm{~ns}$, and the data are collected along the contact surface. A key issue here is how to determine the rebound velocity of the particle.

During the Cold Spray deposition process, the initial kinetic energy would mainly become the elastic strain energy stored in the contact bodies (ALLSE), the plastic strain energy to deform the material plastically (ALLPD) and the energy for the stress wave to propagate. Since the plastic deformation is irreversible, during the restitution, only the stored elastic energy can be recovered as the rebound kinetic energy. Portions of the kinetic energy would be dissipated by unrecoverable plastic deformation and stress wave 
(a)

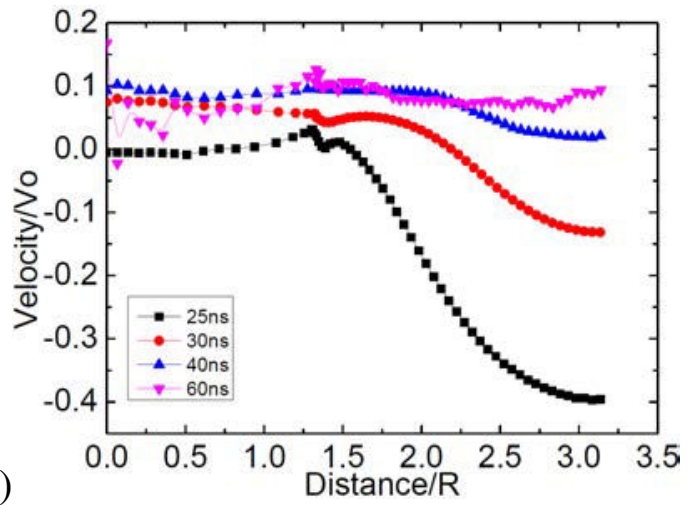

(b)

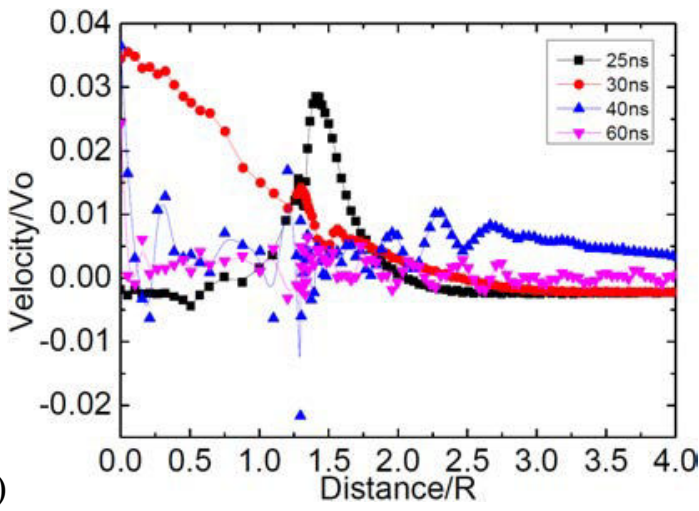

Figure 3.29: Rebound velocities of (a) Al particle and (b) Al substrate at $500 \mathrm{~m} / \mathrm{s}$.

propagation. It is shown in FIG.3.30, the initial kinetic energy is dissipated within the particle and transferred into the substrate as plastic strain energy, and generates plastic deformations, which $96 \%$ of strain energy (ALLIE) is composed by plastic strain energy (ALLPD). The initial kinetic energy loss due to stress wave propagation is only less than $4 \%$.

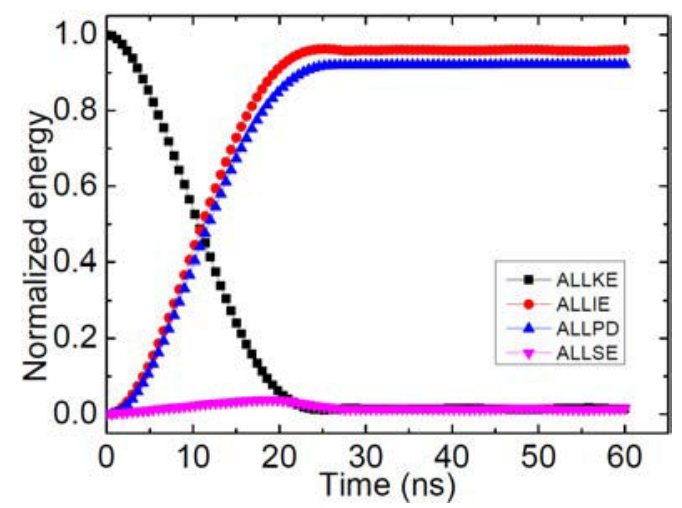

Figure 3.30: Time histories of normalized kinetic energy of $\mathrm{Al} / \mathrm{Al}$ by using the CEL numerical approach at $500 \mathrm{~m} / \mathrm{s}$. ALLKE - Kinetic energy; ALLIE - Total strain energy; ALLPD - Energy dissipated by rate-independent and rate-dependent plastic deformation; ALLSE - Recoverable strain energy.

Therefore, an alternative is to determine the rebound velocity from the rebound kinetic energy as follows:

$$
v_{r}=\sqrt{\frac{2 E_{r}}{m}}
$$

Where $E_{r}$ and $m$ stand for rebound kinetic energy and mass of particle, respectively. The total rebound kinetic energy includes the oscillating energy (FIG.3.26(b)) and so the rebound velocity calculated in this way will be slightly higher than the true value. 
Compared with the total rebound energy the oscillating energy, however, is very small and its significance can be neglected. Thus the restitution coefficient could be written as:

$$
e=\sqrt{\frac{E_{r}}{E_{i}}}
$$

Where the $E_{i}$ stands for initial kinetic energy. The restitution coefficient is shown in FIG.3.31. In the higher initial particle temperature cases, the rebounding of the particle is not observed during the calculation time of $60 \mathrm{~ns}$, so the calculation time is prolonged to $100 n s$ in order to get the rebounding kinetic energy.

(a)
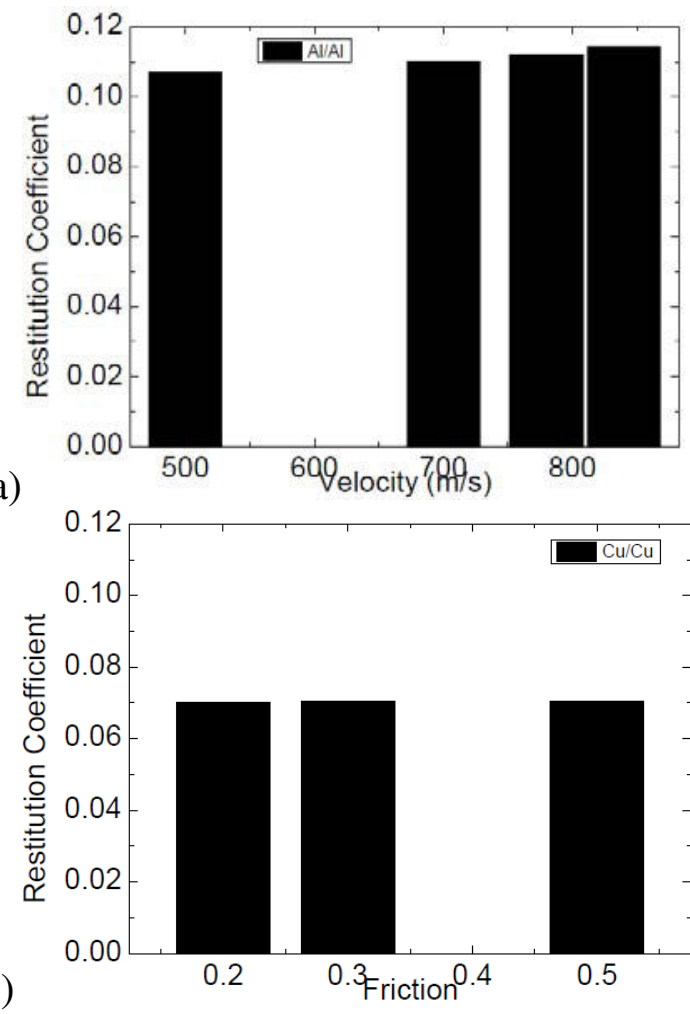

(b)
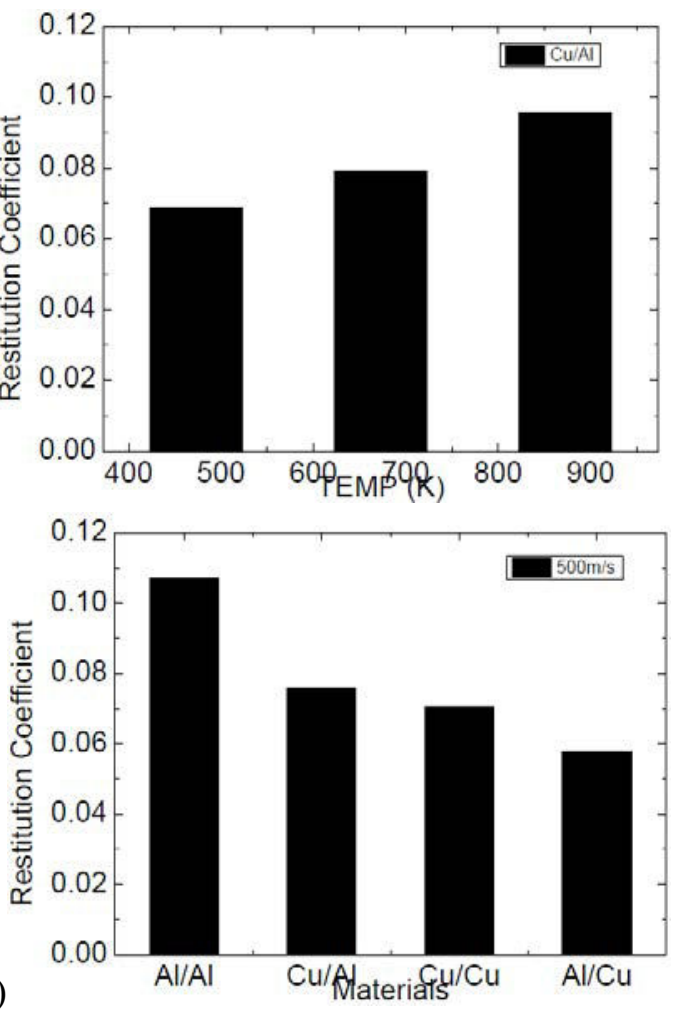

Figure 3.31: Restitution coefficients of (a) different impact velocities, (b) different initial particle temperatures, (c)different friction coefficient and (d) different material combinations.

Obviously, the most significant factor which influences the restitution coefficient is material (FIG.3.31d). Dissimilar material combination has a lower restitution coefficient than that of similar material combination. The friction coefficient has the weakest effect on the restitution coefficient as the FIG.3.31c shows. The restitution coefficient increases slightly along the increasing of impact velocity, this may due to the strain hardening of the material. Anyway, the rebound velocity is approximately constant, which implies the material of the particle yields, deforming plastically. It must be pointed out that if the impact velocity is well lower the critical velocity $(<200 \mathrm{~m} / \mathrm{s})$, then the restitution coefficient increases linearly with the impact velocity; if the impact velocity is well above 
the critical velocity $(>1500 \mathrm{~m} / \mathrm{s})$, the restitution coefficient decreases at higher impact velocities due to the deformations propagating so fast that the material lost its ability to store elastic energy, or material damage at some circumstance [KLI 05]. Between these two limits the rebound velocity is approximately constant as FIG.3.31a shows. The elevating of initial particle temperature also leads to a rise of the restitution coefficient. The mechanical property of a material depends on the temperature, therefore, the particle with higher initial temperature needs less kinetic energy to deform, and it stores more recoverable strain energy consequently. Thus, in a correct simulation, it is necessary to take into account both the working hardening and thermal softening of the material.

It has to be admitted that the restitution coefficients are all higher than zero, which are deviated from the experimental results in some ways. This is a direct result due to the hard contact pressure-overclosure relationship applied to the interaction property. When surfaces are in contact, any contact pressure can be transmitted between them. The surfaces separate if the contact pressure reduces to zero. The interaction between the particle and substrate is not only affected by the pressure, it also involved the substrate surface morphology and harness, van der Waals or electrostatic forces, topochemical reactions, and the repeated impacts coming from the other particles, etc.. The adhesive interactive model, therefore, will be considered to add to the current model for a more precise simulation of interaction.

\subsection{Summary}

The CEL numerical approach has the advantage of catching the particle and substrate deformation both in the solid and viscous states, leading a good correlation with experimental deformed shape of particles as observed at various impact situations.

The material of particle predominantly affects the deposition process and deformation than that of the substrate. Dissimilar material combination has a lower restitution coefficient than that of similar material combination. ТАВ.3.3 presented below schematically demonstrates the effects of the parameters, including velocity, initial particle temperature and friction coefficient, on the outputs we analyzed in this chapter.

\begin{tabular}{|c|c|c|}
\hline Parameters & PEEQ & $\Delta$ TEMP \\
\hline$\uparrow$ Velocity & $\uparrow \uparrow$ & $\uparrow \uparrow$ \\
$\uparrow$ Temperature & $\uparrow \uparrow$ & $\uparrow$ \\
$\uparrow$ Friction & $\uparrow$ & $\rightarrow$ \\
\hline
\end{tabular}

Table 3.3: Schematic illustration of the effects of parameters including Velocity, Temperature and Friction coefficient.

All the parameters studied in this chapter, including velocity, temperature, friction coefficient and material, do not influence the cold spray process independently, actually, they have mutual effects on each other. Taking the velocity and temperature as an example. 
Since particles at higher temperatures need less kinetic energy to plastically deform, properly increasing the particle temperature should allow one to decrease the particle velocity necessary to achieve the same deposition efficiency of the coating, even better quality of the coating. Therefore a lower critical velocity (minimum velocity to avoid particle rebound) may be expected by heating the particles prior to impact. This is in agreement with the experiments of Lee et al.[LEE 07], who showed experimentally that the critical velocity decreases by $50 \mathrm{~m} / \mathrm{s}$ as the process gas temperature increases by $100 \mathrm{~K}$. 


\section{Chapter 4}

\section{Simulation of multiple impacts}

The CEL numerical approach for the analysis of impact behaviors of particle and the substrate has been extensively described in the previous chapter. The calculation of multiple impacts process is of great importance in the clarification of the bonding mechanism as well as the in-depth analysis of coating properties in cold spray. It will be shown in this chapter that, it is possible to estimate the porosity rate and residual stress by using an FCC-like particles arrangement model. The analyses of deformation shape, porosity rate and residual stress, could be combined together to obtain a preliminary understanding of cold spray coating properties. The influences of impact velocity, initial particle temperature and particle size are also discussed in this chapter.

\section{Contents}

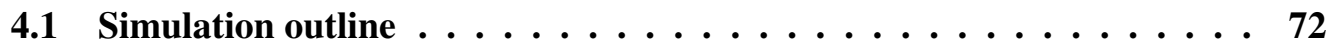

4.2 Deformation shape $\ldots \ldots \ldots \ldots \ldots \ldots \ldots \ldots \ldots$

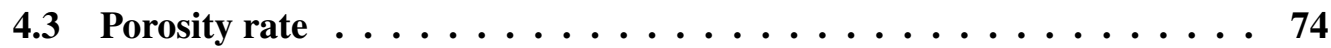

4.4 Residual stress $\ldots \ldots \ldots \ldots \ldots \ldots$

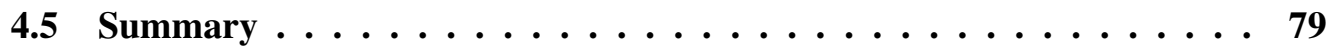




\subsection{Simulation outline}

Traditionally, modeling of cold spray deposited particles has largely focused on systems of one or just a few particles impacting onto a substrate with a view to determining the final shape of the particles after impact. Unlike these traditional approaches, we present simulations of cold spray coating herein with alternative perspectives, which are porosity rate and residual stresses. TAB.4.1 gives the brief outline of the processing conditions for multiple particle impact in this study, focusing on the parameters of impact velocity, initial temperature of particle, particle size and materials. The materials, interaction, analysis procedure, boundary condition and mesh are the same as discussed in the previous chapter.

\begin{tabular}{|c|c|c|c|c|}
\hline Case & Materials & Size $(\mu m)$ & Temperature $(K)$ & Velocity $(\mathrm{m} / \mathrm{s})$ \\
\hline 1 & $\mathrm{Al} / \mathrm{Al}$ & 15 & 413 & 550 \\
2 & $\mathrm{Al} / \mathrm{Cu}$ & 15 & 413 & 550 \\
3 & $\mathrm{Cu} / \mathrm{Cu}$ & 6 & 473 & 600 \\
4 & $\mathrm{Cu} / \mathrm{Al}$ & 6 & 473 & 600 \\
5 & $\mathrm{Cu} / \mathrm{Al}$ & 6 & 473 & 650 \\
6 & $\mathrm{Cu} / \mathrm{Al}$ & 6 & 523 & 600 \\
7 & $\mathrm{Cu} / \mathrm{Al}$ & 6 & 523 & 650 \\
\hline
\end{tabular}

Table 4.1: Calculation plan for investigating multiple impacts.

Considering the computational cost and the continuity feature of coating, it's not necessary to build an arrangement model containing thousands of particles. Inspiring by the cubic crystal system, i.e. each atom at a lattice point parallels to the single particle of powder flow, a Face-Center-Cubic (FCC) particles arrangement model (FIG.4.1c) is used due to its lowest porosity rate of $26 \%$ compared to the Simple Cubic model (FIG.4.1a) and Body-Center-Cubic model (FIG.4.1b), which are $47.7 \%$ and 32\%, respectively. Regarding the particle flow contains different size of particle, the blank space between two particles is filled with another smaller particle as FIG.?? presented, whose size is $2 / 5 D_{p}$. Therefore, the porosity rate of this new particles arrangement model is $21.3 \%$, and hereinafter referred to as a FCC-like model. The dimensions and original mesh are displayed in the FIG.4.3. The boundary of Eulerian material does not have to match element geometry at any time during the analysis and has to be recomputed in each time increment as the material flows through the mesh, the dimensions of Eulerian domain must be large enough (see FIG.4.3) to allow the material to flow freely, meanwhile to prevent the loss of materials after the impact. In order to save the computational cost, the Eulerian domain scales down following the dimensions in the brackets when the particle size decreases from $15 \mu m$ to $6 \mu m$.

It should be mentioned that, the model of multiple particles is different from the actual situation. SAMAREH [SAM 07] developed a MATLAB code to simulated the footprint of more than 5000 particles with different size. His results, as shown in FIG.4.4, inspired us to combine the two different codes together in order to reproduce a more real deposition 
process.
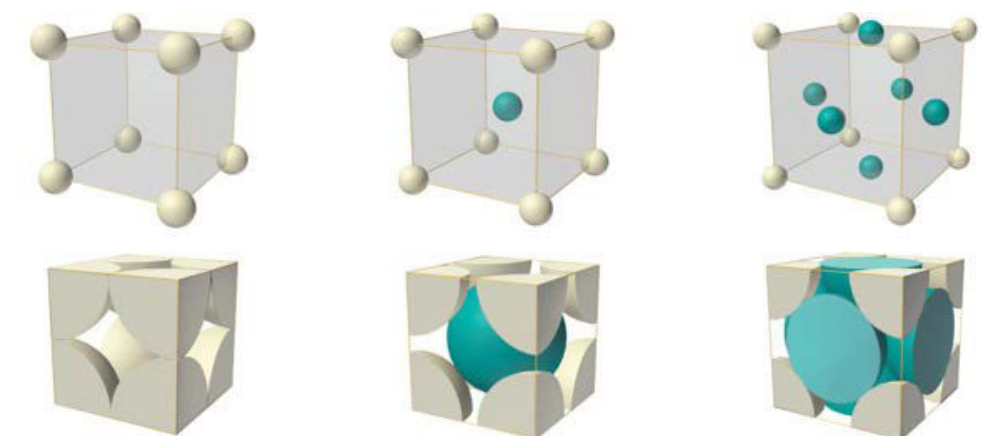

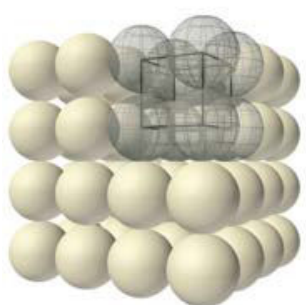

(a) Simple cubic

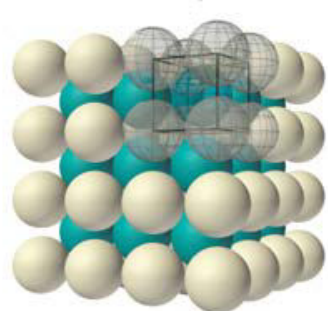

(b) Body-centered cubic

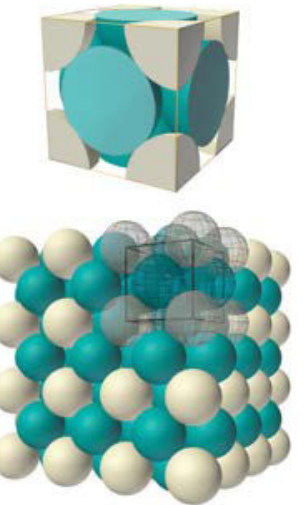

(c) Face-centered cubic

Figure 4.1: Schematic diagrams of three kinds of cubic unit cell, which are (a) simple cubic, (b) body center cubic and (c) face center cubic.

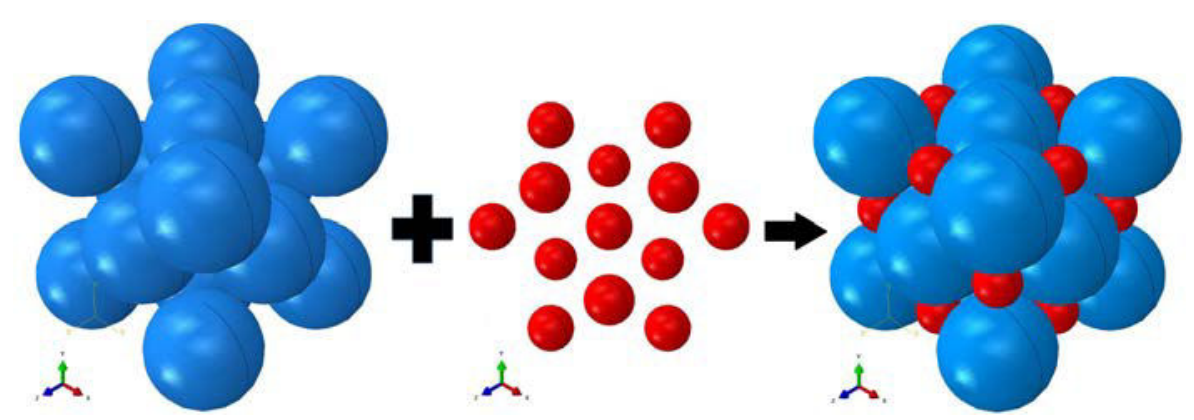

Figure 4.2: Schematic diagram of the combination of two FCC particles arrangement models.

\subsection{Deformation shape}

The deformation behaviors of $\mathrm{Al}$ particles and $\mathrm{Cu}$ particles at different processing conditions obtained from experiments [LUZ 11] and modeled by CEL approach are shown in Fig.4.5 and Fig.4.6, respectively. Microscopically, both coatings are uniform and free of large voids or cracks, and the overall structure is granular. Simulation results give some details about the deformation of particles. Firstly, the interface between particles in the Eulerian domain is almost indistinguishable. Within each time increment, the 


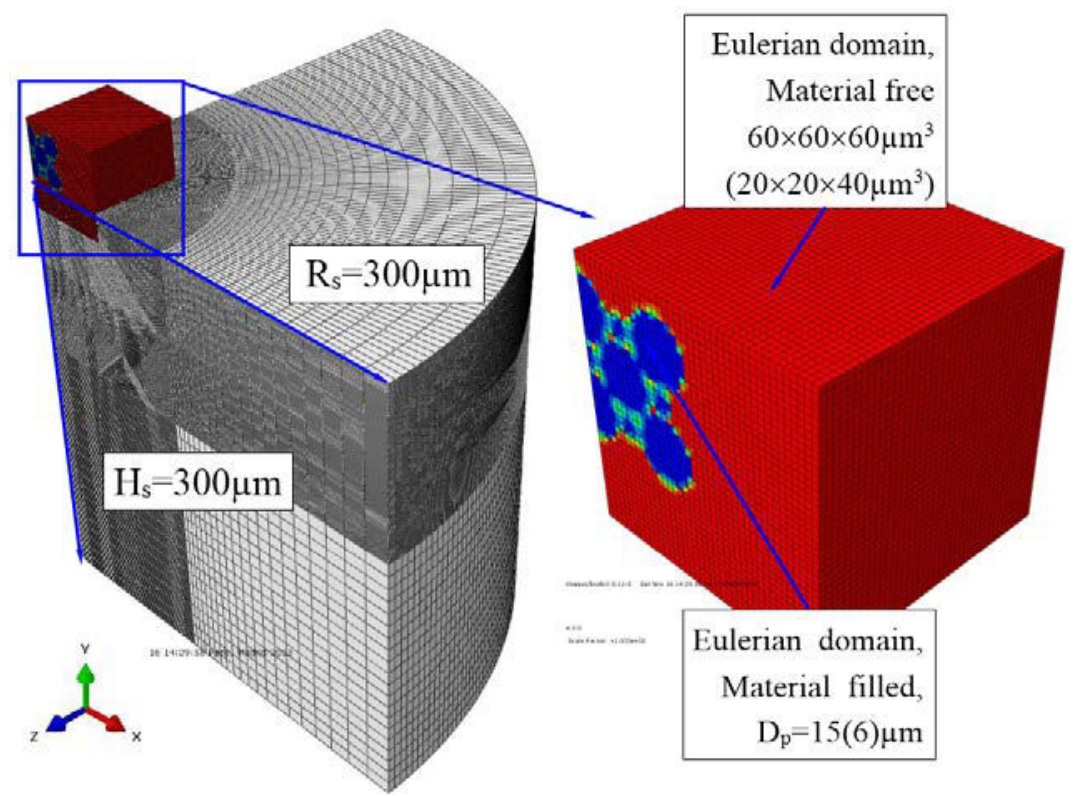

Figure 4.3: Schematic diagram of the 3D CEL model with quarter symmetry.

boundaries of each Eulerian material are reconstructed using the volume fraction data of each Eulerian element. The interface reconstruction algorithm approximates the material boundaries within an element as simple planar facets. This assumption produces a simple, approximate material surface that may be discontinuous between neighboring elements. Therefore, accurate determination of a location of material within an element is possible only for simple geometries, and fine grid resolution is required in most Eulerian analyses. The scattered green elements, which EVF equals to 0.50, reveal the interface between particles and substrate implicitly. As can be seen from FIG.4.6, prior deposited particles are tamped to irregular shapes by further impact of subsequent incident particles, while particles at the top layer deform like a single particle does.

The estimated impact parameters, including contact time, maximum equivalent plastic strain $\left(\mathrm{PEEQ}_{\max }\right)$, maximum temperature $\left(\mathrm{TEMP} \mathrm{P}_{\max }\right)$, mean strain rate $\left(\mathrm{ER}_{\text {mean }}\right)$, maximum contact pressure $\left(\mathrm{CPRESS}_{\max }\right)$ and restitution coefficient $(e)$, are presented in ТАВ.4.2. All the data are selected during the contact time that is defined as the time period from the beginning to the moment which contact pressure on whole contact area equals to 0 .

Under the process conditions of case 1 to 4 mentioned in TAB.4.1, the contact time of similar combination $(\mathrm{Al} / \mathrm{Al}$ and $\mathrm{Cu} / \mathrm{Cu})$ is shorter than that of dissimilar combination. The longest contact time is $94 n s$ for $\mathrm{Cu} / \mathrm{Al}$ case due to the deepest crater of substrate produced by $\mathrm{Cu}$ particles. The center of particles starts to rebound since $38 n s$, but the whole detachment of particles from the substrate costs another 56 ns. Al substrate (Al/Al and $\mathrm{Cu} / \mathrm{Al}$ cases) undergoes larger amount plastic deformation than $\mathrm{Cu}$ substrate. $\mathrm{Al}-$ though the $\mathrm{Cu}$ particles have a higher initial temperature, the $\mathrm{Al} / \mathrm{Al}$ case still obtains a higher value of temperature $(500.5 \mathrm{~K})$ due to its lower specific heat compared to $\mathrm{Cu}$ ma- 


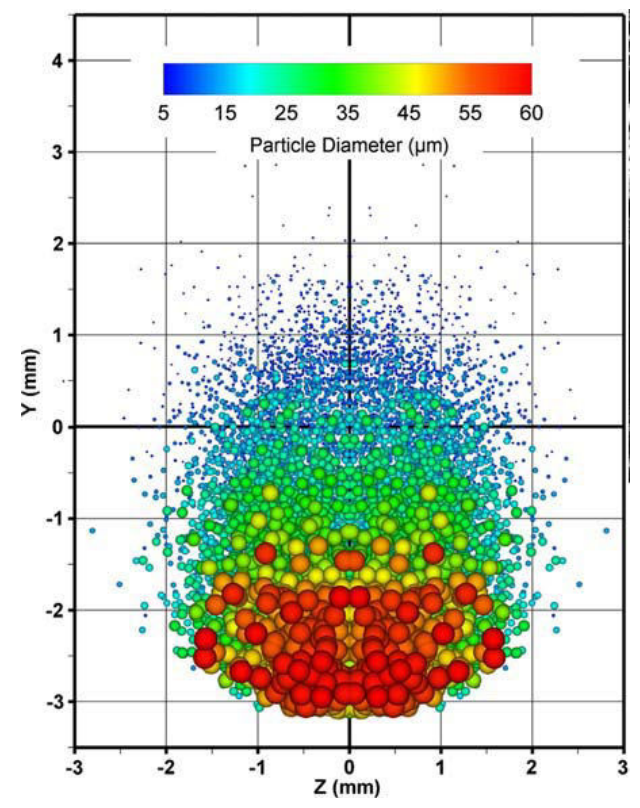

Figure 4.4: Landing location for a particle range between 5 and $60 \mu \mathrm{m}$ for a flat substrate, placed at a standoff distance of $10 \mathrm{~mm}$ [SAM 07].

terial. The impact duration, which is the time period from the beginning to the moment that particles start to rebound, is very short, i.e. $\sim 40-60 \mathrm{~ns}$, but the average strain rate $\left(\sim 10^{5} s^{-1}\right)$ is quite high, which the local strain rate of particles even reaches to $\sim 10^{6} s^{-1}$. The highest strain rate always occurs at the beginning of contact, and it changes relatively little after $20 \mathrm{~ns}$ of contact. Compared to Al, the higher density and higher impact velocity of $\mathrm{Cu}$ enable a higher initial kinetic energy, inducing a higher strain rate and impact pressure. The maximum contact pressure is obtained usually at the first moment of contact, and then it decreases over time. For $\mathrm{Cu} / \mathrm{Cu}$ case, the maximum contact pressure of 12.22 $\mathrm{GPa}$ is obtained at $2 \mathrm{~ns}$. The restitution coefficients of multiple impacts with the different materials group agree well with the results presented in the last chapter, which the minimum restitution coefficient is found in $\mathrm{Al} / \mathrm{Cu}$ case, and the maximum one is found in $\mathrm{Al} / \mathrm{Al}$ case.

\subsection{Porosity rate}

Following the legend colors which correspond to the volume fraction of integration points, where the blue color represents an Eulerian element filled with material, and where, on the contrary, the red color means that the element is empty or fill with voids, it could observe several empty elements in some areas at the interface between particles (red elements displayed in FIG.4.6). These empty elements, in other words, are pores or cavities showing that the coating is somewhat porous. FIG.4.7 gives a clearer view about the feature of pores and their distributions within the particles. The size of pores distributed within $\mathrm{Cu}$ particles is smaller, and their shapes are amorphous. It can be seen 
(a)
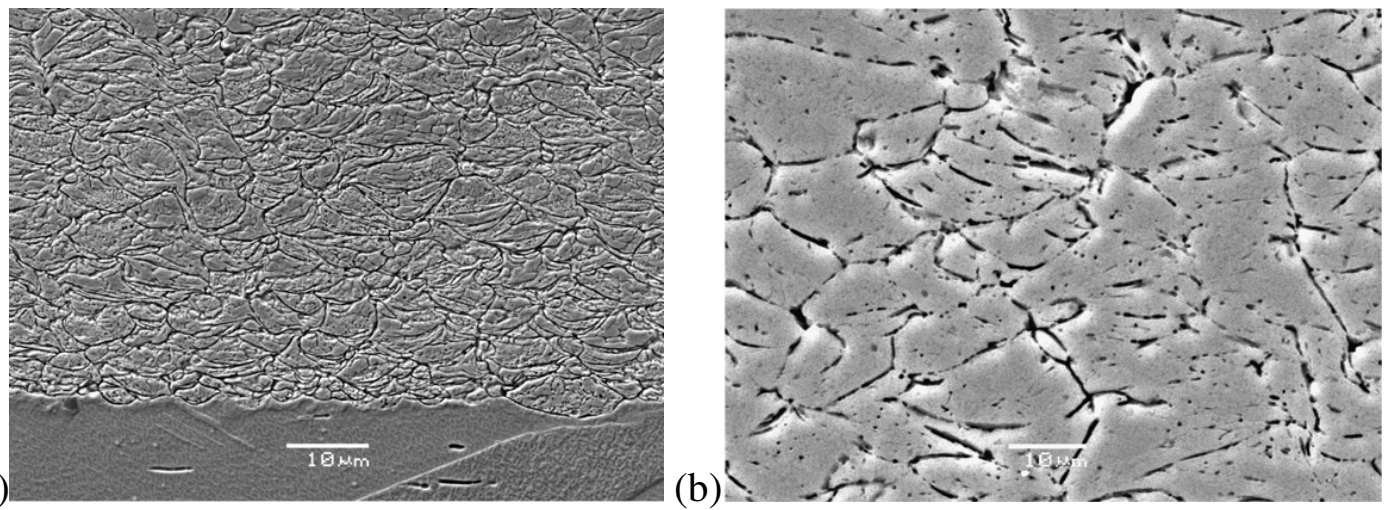

Figure 4.5: Secondary electron micrographs of the cross-section of (a) $\mathrm{Al} / \mathrm{Al}$ and (b) $\mathrm{Cu} / \mathrm{Cu}$.

\begin{tabular}{|c|c|c|c|}
\hline Materials & Contact time $(n s)$ & PEEQ $_{\max }$ & TEMP $_{\max }(K)$ \\
\hline $\mathrm{Al} / \mathrm{Al}$ & 65 & 2.638 & 500.5 \\
$\mathrm{Al} / \mathrm{Cu}$ & 90 & 2.195 & 423.1 \\
$\mathrm{Cu} / \mathrm{Cu}$ & 62 & 2.368 & 452.6 \\
$\mathrm{Cu} / \mathrm{Al}$ & 94 & 2.417 & 468.9 \\
\hline $\mathrm{Materials}$ & $\mathrm{ER}_{\text {mean }}\left(s^{-1}\right)$ & $\mathrm{CPRESS}_{\max }(\mathrm{GPa})$ & $e(\%)$ \\
\hline $\mathrm{Al} / \mathrm{Al}$ & $4.88 \times 10^{5}$ & 5.46 & 15.61 \\
$\mathrm{Al} / \mathrm{Cu}$ & $4.29 \times 10^{5}$ & 7.28 & 8.25 \\
$\mathrm{Cu} / \mathrm{Cu}$ & $8.79 \times 10^{5}$ & 12.22 & 8.50 \\
$\mathrm{Cu} / \mathrm{Al}$ & $9.46 \times 10^{5}$ & 6.55 & 11 \\
\hline
\end{tabular}

Table 4.2: Estimated impact parameters based on the simulations of CEL numerical approach.

from these two figures that, the volume fraction of porosity in the $\mathrm{Cu}$ particles is lower than that of $\mathrm{Al}$ particles.

The method of calculating porosity rate in this study is quite different from the experimental methods. It is very difficult to calculate the exact volume of pores due to their irregular shapes, so this method is just an approximation. Because the mesh structure of Eulerian domain does not change during the simulation and all the element has the same volume, so the volume of pores could be obtained by the number of void elements times the element volume. Then the porosity rate could be obtained approximately as:

$$
\text { Porosity rate }=\frac{\text { number of void elements }}{\text { number of total elements }} \times 100 \%
$$

In this study, instead of the whole volume of deformed particles, one smaller cubic volume is chosen for measuring the porosity rate in order to eliminate the effect of the outer surface of particles. Because in some local area of the outer surface of particles, the EVF of element may approach to 1; when counting the number of void elements, these surface elements will be included unavoidably. 


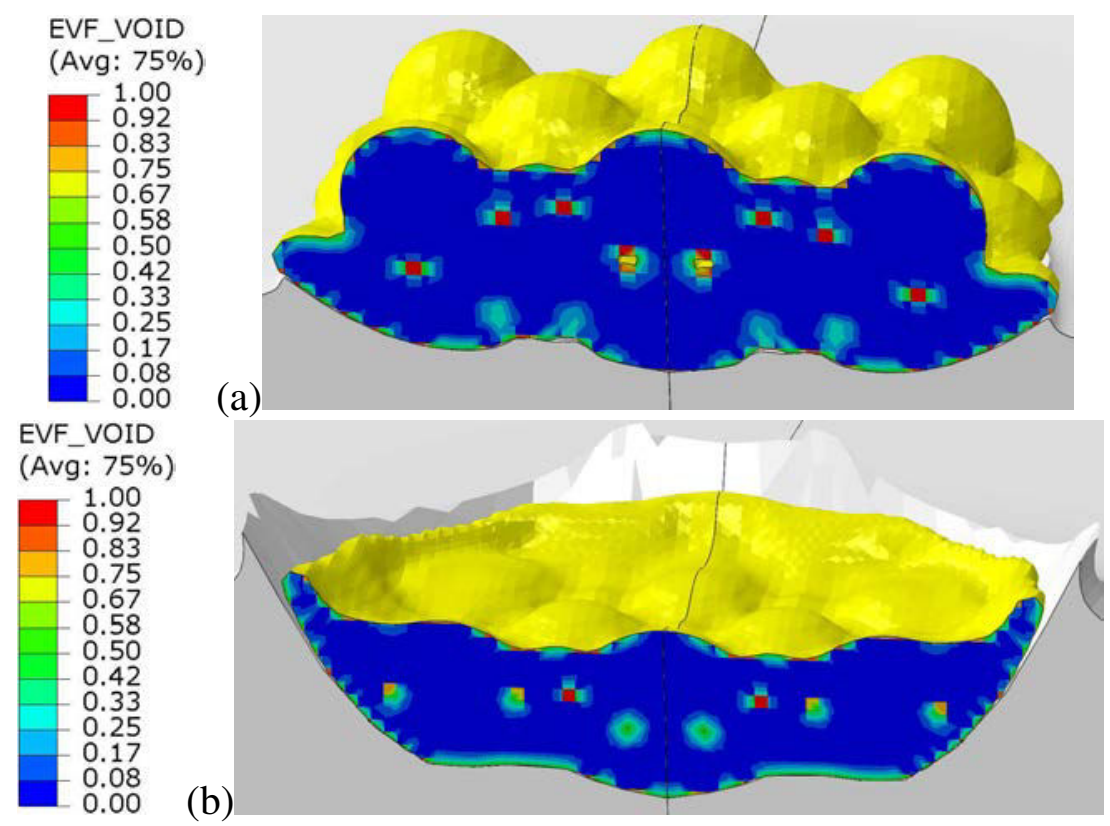

Figure 4.6: Simulation profiles of (a) $\mathrm{Al} / \mathrm{Al}$ and (b) $\mathrm{Cu} / \mathrm{Cu}$ by using the $\mathrm{FCC}$ particles arrangement model.

The first step is to cut the deformed particles into a cubic volume as the FIG.4.8 shows. Separating the elements whose EVF range from 0.9 to 1 is the second step, using the 'Result value' method available in the 'Display group' tool of ABAQUS/CAE. The 'Query' tool is used to count all void elements in the third step. The number of total elements could be obtained by counting all the active elements in the Eulerian domain presented in FIG.4.8b through the same manner.

The estimated porosity rates $\left(\mathrm{PR}_{\mathrm{sim}}\right)$ by using the Equation [?] and method explained above are displayed in TAB.4.3, comparing with the experiment results $\left(\mathrm{PR}_{\exp }\right)$ [LUZ 11] presented in the last column of TAB.4.3. The results clearly show that a higher density of $\mathrm{Cu}$ enable it to form a higher integrity coating than $\mathrm{Al}$. The porosity rate of $\mathrm{Al} / \mathrm{Cu}$

(a)

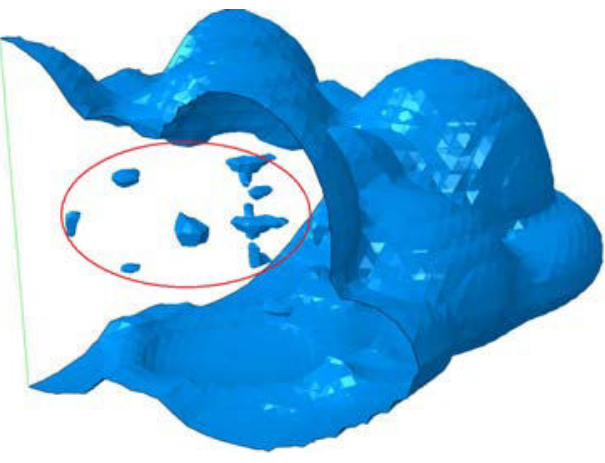

(b)

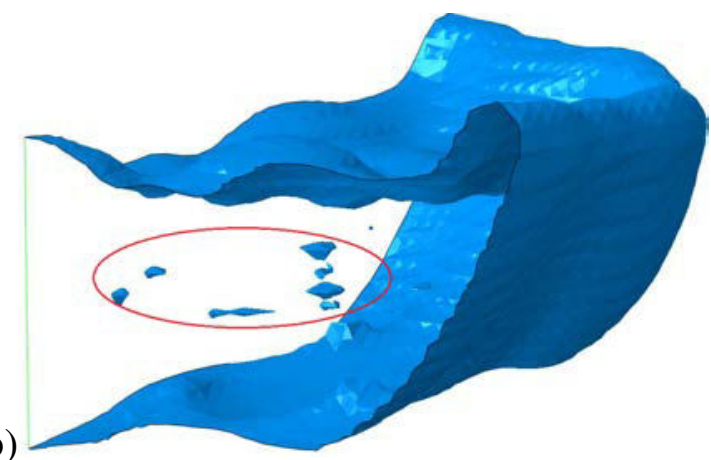

Figure 4.7: Simulation contours of pores for (a) $\mathrm{Al} / \mathrm{Al}$ and (b) $\mathrm{Cu} / \mathrm{Cu}$ by using the FCC particles arrangement model. 
case is more than two times of $\mathrm{Cu} / \mathrm{Cu}$ case. The simulation results agree well with the experiment results, comparing the impact cases of $\mathrm{Al} / \mathrm{Cu}$ and $\mathrm{Cu} / \mathrm{Cu}$.

The impact case 4 of $\mathrm{Cu} / \mathrm{Al}$ obtains the lowest porosity rate $(2.966 \%)$, so this materials group is used to study the influences of velocity and temperature on the porosity rate. The features of voids under the different process conditions are shown in FIG.4.10. It is clear from FIG.4.10b - d, that the volume of pore is smaller, and the pores at the same position are elongated, and some pores disappear like the one close to the right limit of red ellipse figure marked in the FIG.4.10a. TAB.4.1 presents the corresponding data of estimated porosity rate. As the result of case 5 shows that, the porosity rate decreases $11.78 \%$ if the impact velocity increases $50 \mathrm{~m} / \mathrm{s}$. As have discussed in the last chapter, the higher impact velocity, the larger amount of plastic deformation, which indicated the aspect ratio of particles increase with increasing velocity. The decline in volume fraction of the porosity is most due to this larger plastic deformation of the particles. Increasing initial temperature of particles will also reduce the voids, as the result of case 6 illustrates that the porosity rate decreases $6.73 \%$ if the initial temperature rises $50 \mathrm{~K}$. Due to the thermal softening effect, the material of particles undergo larger deformation when the temperature approaching the melting temperature. A straightforward of this phenomenon is the decreasing of the void size, and thus the density of cavity. The porosity rate of case 7 is a result produced by the mutual effect of velocity and temperature. This case produces the minimum porosity rate $2.32 \%$ as anticipating.

The formation mechanism of pores considered in this study is only based on the concept of augmentation of particles, however, there is still other factors which may provoke the initiation of pores. BASSIM [BAS 08] tested the materials in compression at strain rates ranging between $10^{3}$ and $10^{4} s^{-1}$ using direct impact Hopkinson Pressure Bar. He found out that plastic deformation in the materials is dominated by shear strain localization along adiabatic shear bands (ASBs). Cracks are initiated and propagated along these bands leading to failure as shown in FIG.4.11a. BENZERGA [BEN 10] observed the void nucleation from a macroscopic scale (FIG.4.11b), which is also caused by the extreme plastic deformation. It would be interesting to study further the influence of different mechanism on the formation of pores in the cold spray coating.

(a)

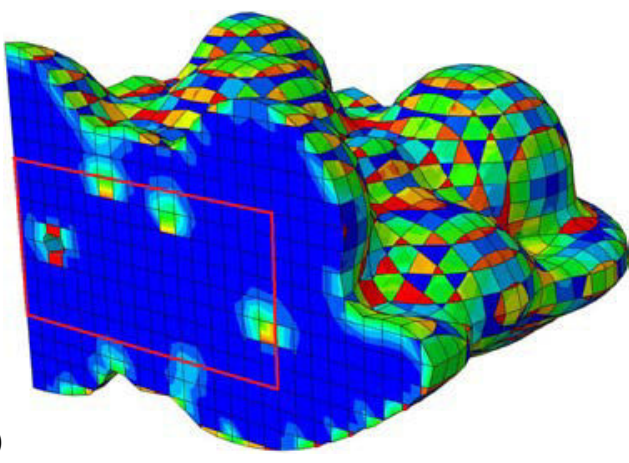

(b)

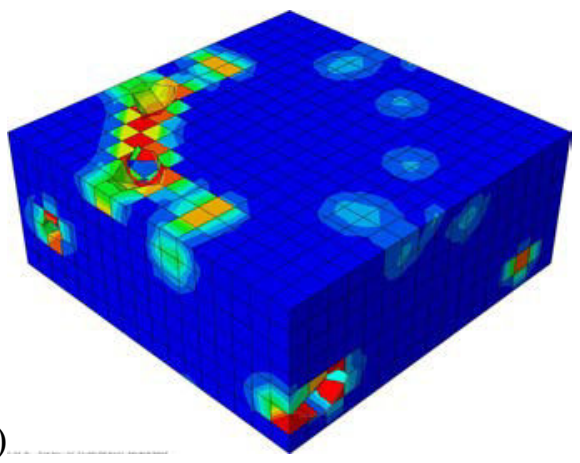

Figure 4.8: Schematic diagrams of (a) deposited particles and (b) cubic volume part for calculating porosity rate. 


\begin{tabular}{|c|c|c|c|}
\hline Case & Materials & $\mathrm{PR}_{\text {sim }}$ (Vol.\%) & $\mathrm{PR}_{\exp }$ (Vol.\%) \\
\hline 1 & $\mathrm{Al} / \mathrm{Al}$ & 5.41 & N/A \\
2 & $\mathrm{Al} / \mathrm{Cu}$ & 6.03 & $6.4 \pm 0.6$ \\
3 & $\mathrm{Cu} / \mathrm{Cu}$ & 2.97 & $1.5 \pm 0.5$ \\
4 & $\mathrm{Cu} / \mathrm{Al}$ & 2.97 & N/A \\
5 & $\mathrm{Cu} / \mathrm{Al}$ & 2.62 & N/A \\
6 & $\mathrm{Cu} / \mathrm{Al}$ & 2.77 & N/A \\
7 & $\mathrm{Cu} / \mathrm{Al}$ & 2.32 & N/A \\
\hline
\end{tabular}

Table 4.3: comparison of predicted volume porosity rate and experiment results.

\subsection{Residual stress}

Coating integrity may be loosely defined as the quality of bonding of between particles within the coating, and between the coating and the substrate [LUZ 11]. Coating integrity is influenced by the residual stresses present in the coating. Given the complexity of the cold spray process, a finite element simulation is best able to capture details the mechanics of stress accumulation. As have introduced in the first chapter, quench, thermal mismatch and peening stresses are all presented in cold sprayed coatings. It is hard to predict the thermal mismatch stress due to the limit of calculation time, which means the substrate-particles system can not be cooled down to room temperature in the simulation. Therefore, only the quenching stress and peening stress are considered in this study, and their combination effects is known as evolving stress, which represents a stress developed when a layer of material is deposited in the substrate [SUH 13].

The simulation results of the through thickness mean stress distributions for different process conditions are shown in FIG.4.12. The data are selected on the axis of symmetry at the end of impact duration, i.e. Y-axis, from the top of particles to the bottom of substrate. Note that the positive stresses represent the compressive stresses in this study, and thus the negative ones represent the tensile stresses. Meanwhile, the positive distance stands for the deformed particles part, and the negative distance stands for the substrate part; then Distance $R=0$ is the position where the interface between particles and substrate locates.

First, a characteristic compressive stress region under the surface of the substrate could be observed, due to the peening stress inducing by large plastic deformation of deposited particles. The maximum compressive stress of substrate produced by Al particles (FIG.4.12a,b) is situated at the depth of around 5R; however, the maximum compressive stress of substrate produced by $\mathrm{Cu}$ particles locates much closer to the substrate surface; and among these four impact cases, the peak value of compressive stress is $841 \mathrm{MPa}$ found in $\mathrm{Cu} / \mathrm{Al}$ impact case. The magnitude of the compressive residual stress reduces after reaching the maximum value along the depth direction, and a small amount of compensatory tensile stress is observed after which the compressive stress becomes negligibly small.

Second, for $\mathrm{Cu}$ particles (FIG.4.12c and d), the whole interface region only contains 
(a)

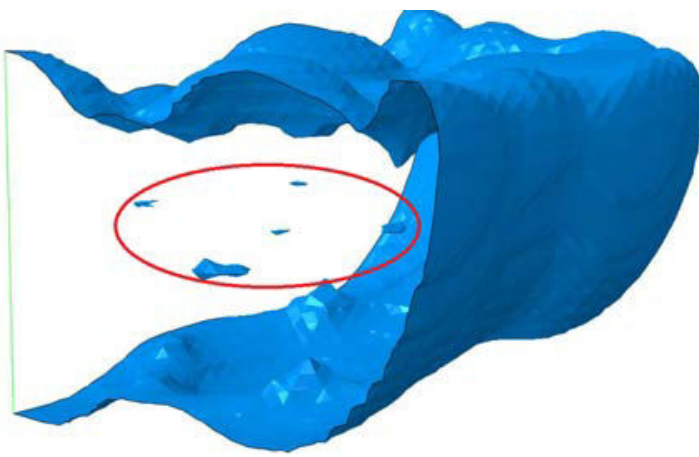

(c)

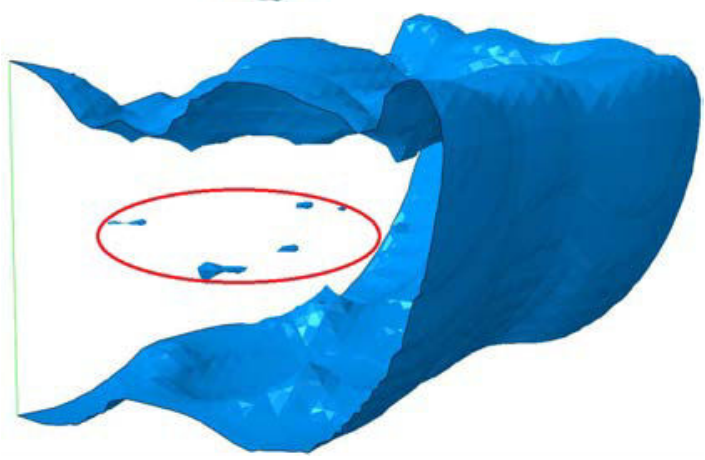

(b)

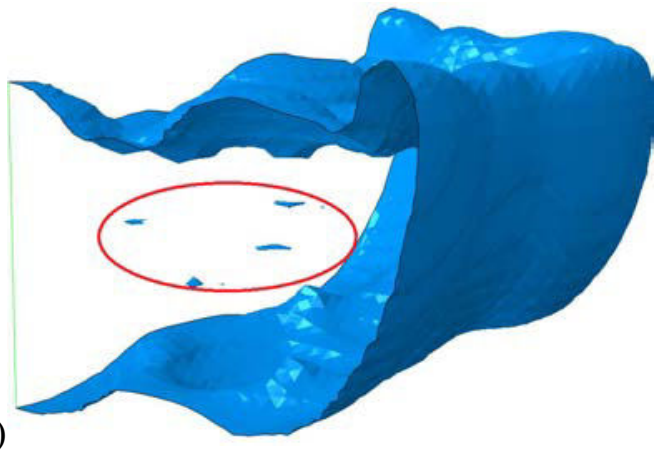

(d)

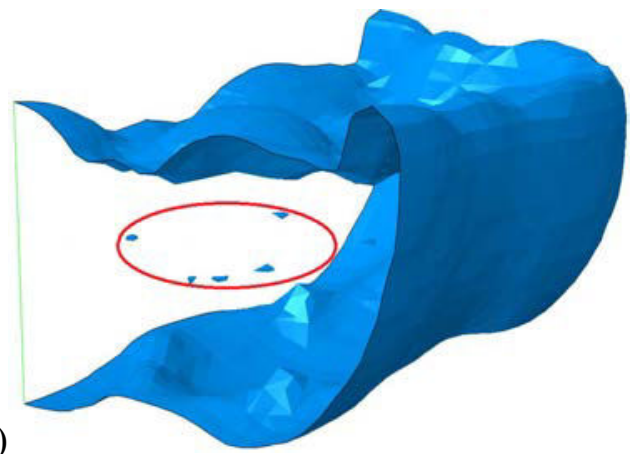

Figure 4.9: Simulation contours of pores for different process conditions of impact case $\mathrm{Cu} / \mathrm{Al}$. (a) case 4 - $600 \mathrm{~m} / \mathrm{s}, 473 \mathrm{~K}$; (b) case 5 - $650 \mathrm{~m} / \mathrm{s}, 473 \mathrm{~K}$; (c) case 6 - $600 \mathrm{~m} / \mathrm{s}$, $523 \mathrm{~K}$; (d) case $7-650 \mathrm{~m} / \mathrm{s}, 523 \mathrm{~K}$.

the compressive stress; for $\mathrm{Al}$ particles, there is tensile stress within the particles, and the tensile stress and compressive stress occur alternately for $\mathrm{Al} / \mathrm{Cu}$ case. One explanation for this behavior is the difference of impact velocity. In our simulation, the impact velocity of Al particle is $550 \mathrm{~m} / \mathrm{s}$, and for $\mathrm{Cu}$ particle is $600 \mathrm{~m} / \mathrm{s}$. The $\mathrm{Cu}$ particles obtain higher kinetic energy, and thus have a larger amount of plastic deformation. As FIG.4.6b illustrated, all the $\mathrm{Cu}$ particles have completely lost their original sphere shape and deformed into a lens-like shape. On the contrary, the top layer of Al particles (FIG.4.6a) has relatively smaller deformation compared to the first layer of particles, and resulting in the tensile stresses distributed among the $\mathrm{Al}$ particles.

Third, the compressive residual stress of substrate produced by $\mathrm{Cu}$ particles is two times higher than that produced by Al particles. The evidently higher values of compressive residual stress in the case of $\mathrm{Cu} / \mathrm{Cu}$ and $\mathrm{Cu} / \mathrm{Al}$ suggest the amount of plasticity on impact is more significant, resulting from the higher initial kinetic energy of $\mathrm{Cu}$ particles. In the case of $\mathrm{Cu} / \mathrm{Cu}$, the interfacial compressive region extended into the substrate to a greater extent of $24 \mathrm{R}$ with a maximum compressive stress adjacent to the interface of approximately $540 \mathrm{MPa}$.

The impact velocity and temperature have significant influences on the distribution of residual stresses. FIG.4.13 displays the simulation results of mean stress distributions for $\mathrm{Cu} / \mathrm{Al}$ with different process conditions. As FIG.4.13b shows, the peak value of compressive residual stress increases $15.9 \%$ when impact velocity increases $50 \mathrm{~m} / \mathrm{s}$; and the depth 
(a)

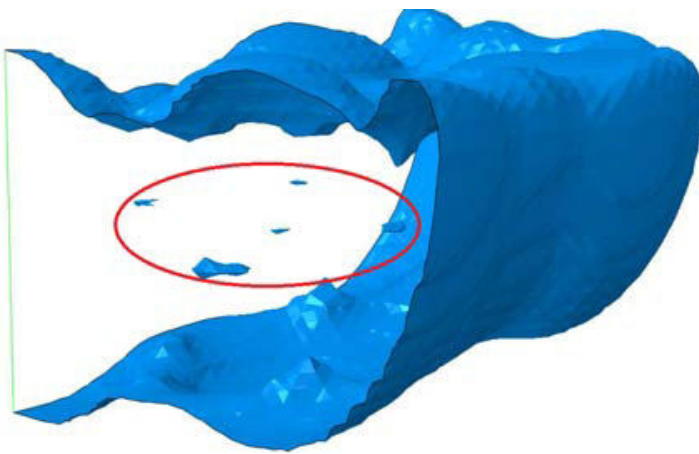

(c)

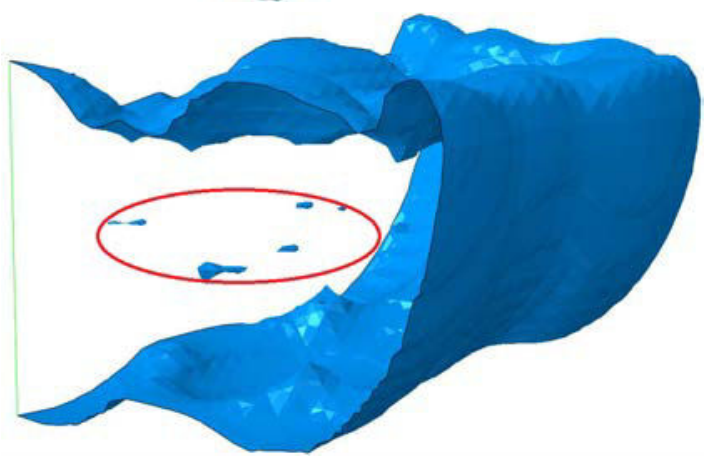

(b)

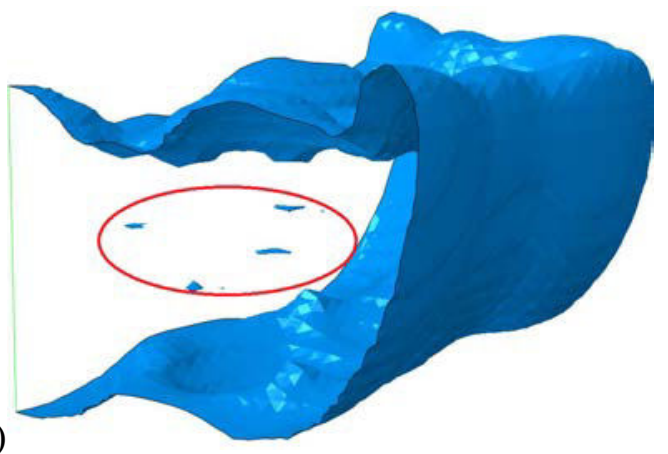

(d)

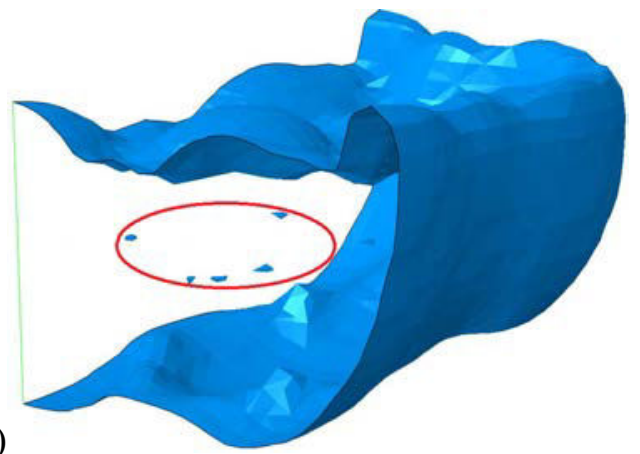

Figure 4.10: Simulation contours of pores for different process conditions of impact case $\mathrm{Cu} / \mathrm{Al}$. (a) case 4 - $600 \mathrm{~m} / \mathrm{s}, 473 \mathrm{~K}$; (b) case 5 - $650 \mathrm{~m} / \mathrm{s}, 473 \mathrm{~K}$; (c) case 6 - $600 \mathrm{~m} / \mathrm{s}$, $523 \mathrm{~K}$; (d) case $7-650 \mathrm{~m} / \mathrm{s}, 523 \mathrm{~K}$.

of compressive residual stress extends 5.23\%. Moreover, the peak value of compressive residual stress increases $8.99 \%$ when initial particle temperature increases $50 \mathrm{~K}$. For the impact case 7 (FIG.4.13d), besides the peak value of compressive residual stress increases $13.15 \%$, the tensile residual stress region is found beneath the depth of $20 \mathrm{R}$ of substrate.

A higher impact velocity leads to a larger amount of plastic deformation, thus the higher compressive residual stress, as well as the higher initial particle temperature. For dissimilar combination as $\mathrm{Cu} / \mathrm{Al}$, particle material at the substrate surface has a relative displacement due to friction effect, resulting in tensile stress. This relative displacement becomes more significant under higher impact velocity and higher initial temperature, therefore the tensile stress at the interface between particle and substrate increases. The rise of tensile stress leads to a declining of compressive stress at the interface between particles and the substrate. Comparing FIG.4.13a and FIG.4.13d, the compressive stress at the interface decreases about $21 \%$.

\subsection{Summary}

The FCC-like model is suitable for the investigation of multiple impacts. It is possible to estimate the porosity rate and residual stresses in function of the material properties and process parameters based on this multiple impacts model. 
(a)

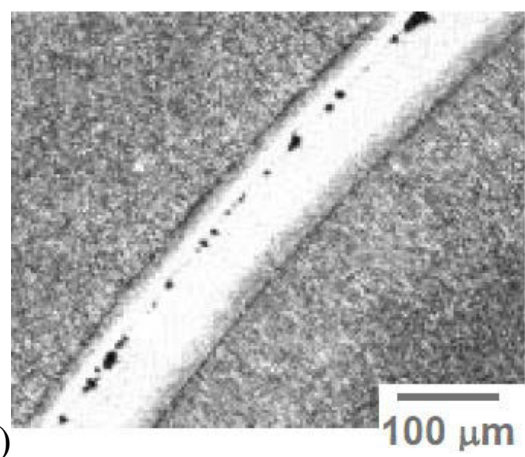

(b)

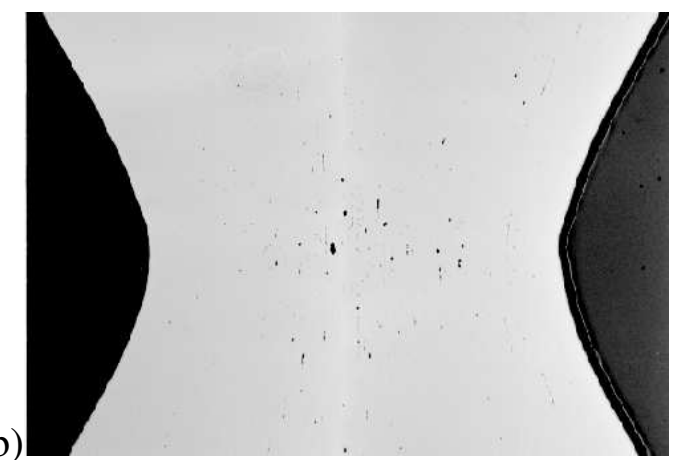

Figure 4.11: (a) Crack initiation along ASB in AISI 4340 steel subjected to high velocity impact [BAS 08], and (b) Void accumulation of ductile fracture in round notched bars of high strength steel [BEN 10].

Thanks to this CEL numerical approach, the feature and distribution of pores could be seen directly. A new method for estimating porosity rate was provided according to the EVF value of Eulerian elements, and the predicted results agree well with the experiment results. The cold spray bonding process is originated from high plastic strain and high plastic strain rate. Therefore, material that undergoes a greater amount of plastic strain ( $\mathrm{Cu}$ in this case) is expected to form a denser coating. The mutual effect of impact velocity and temperature could decrease the porosity rate significantly.

The magnitude and type of residual stresses may vary significantly, depending on the particle/substrate material system. The compressive residual stress induced by cold spray deposition is the manifestation of the different stress generation mechanism. It must be connected not only to the elastic but also, more important, plastic properties of material. The compressive residual stress under the substrate surface increases along with the rise of impact velocity and initial particle temperature.

Further investigation is needed to understand the relationship between the other process parameters except velocity and temperature, and the resultant residual stresses. 

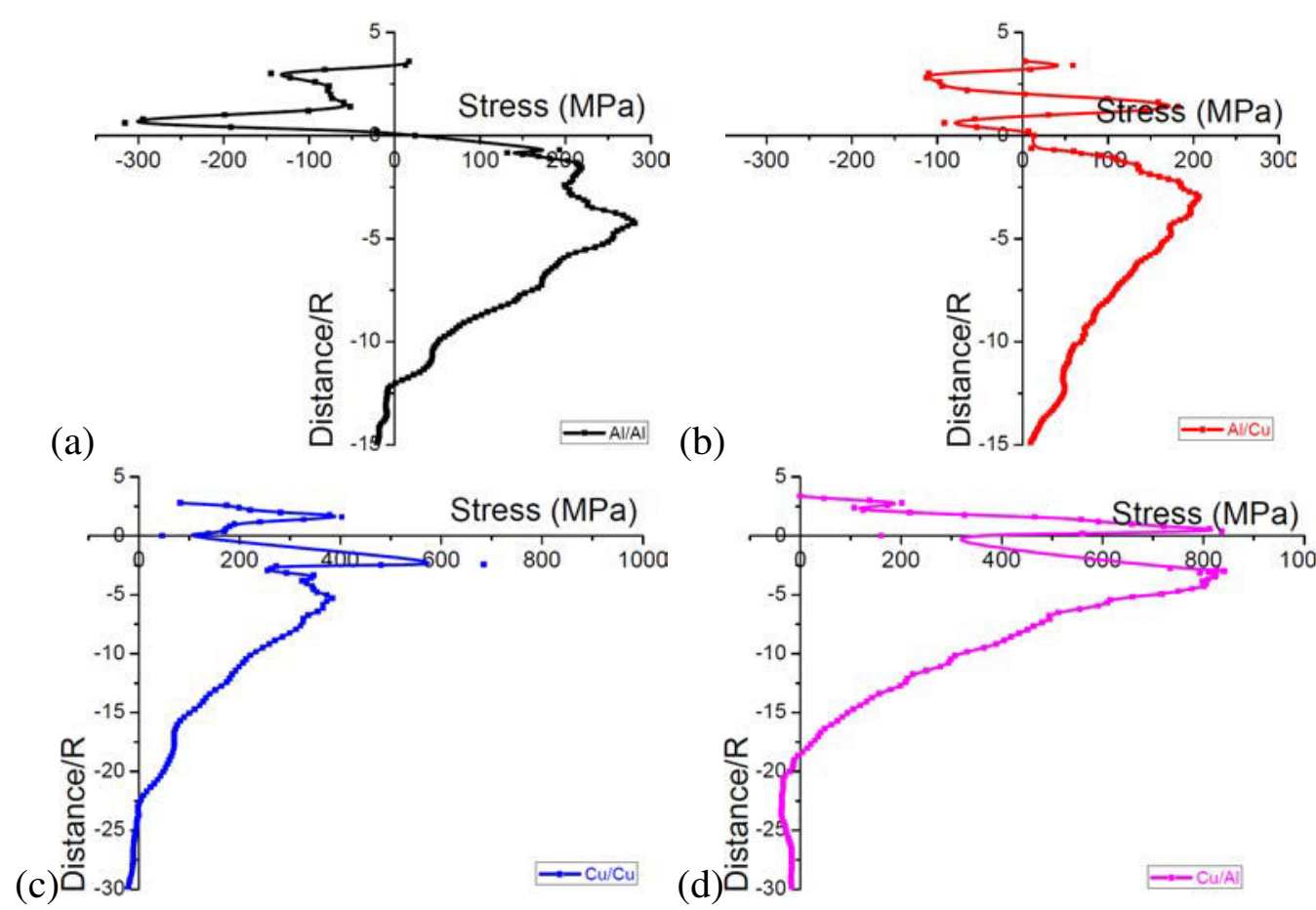

(b)

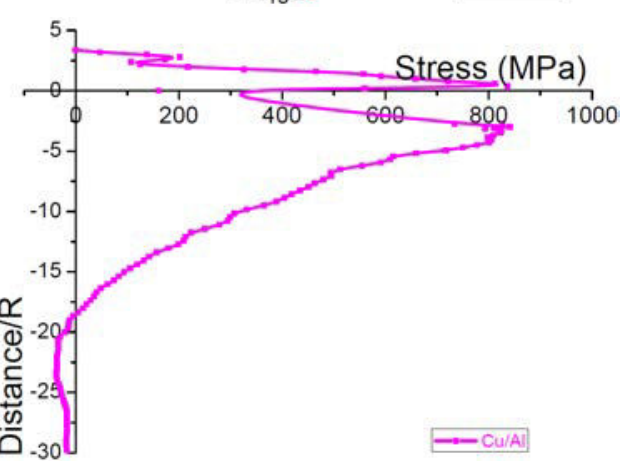

Figure 4.12: Simulation results of the through thickness mean stress distributions for (a) $\mathrm{Al} / \mathrm{Al}$, (b) $\mathrm{Al} / \mathrm{Cu}$, (c) $\mathrm{Cu} / \mathrm{Cu}$ and (d) $\mathrm{Cu} / \mathrm{Al}$.

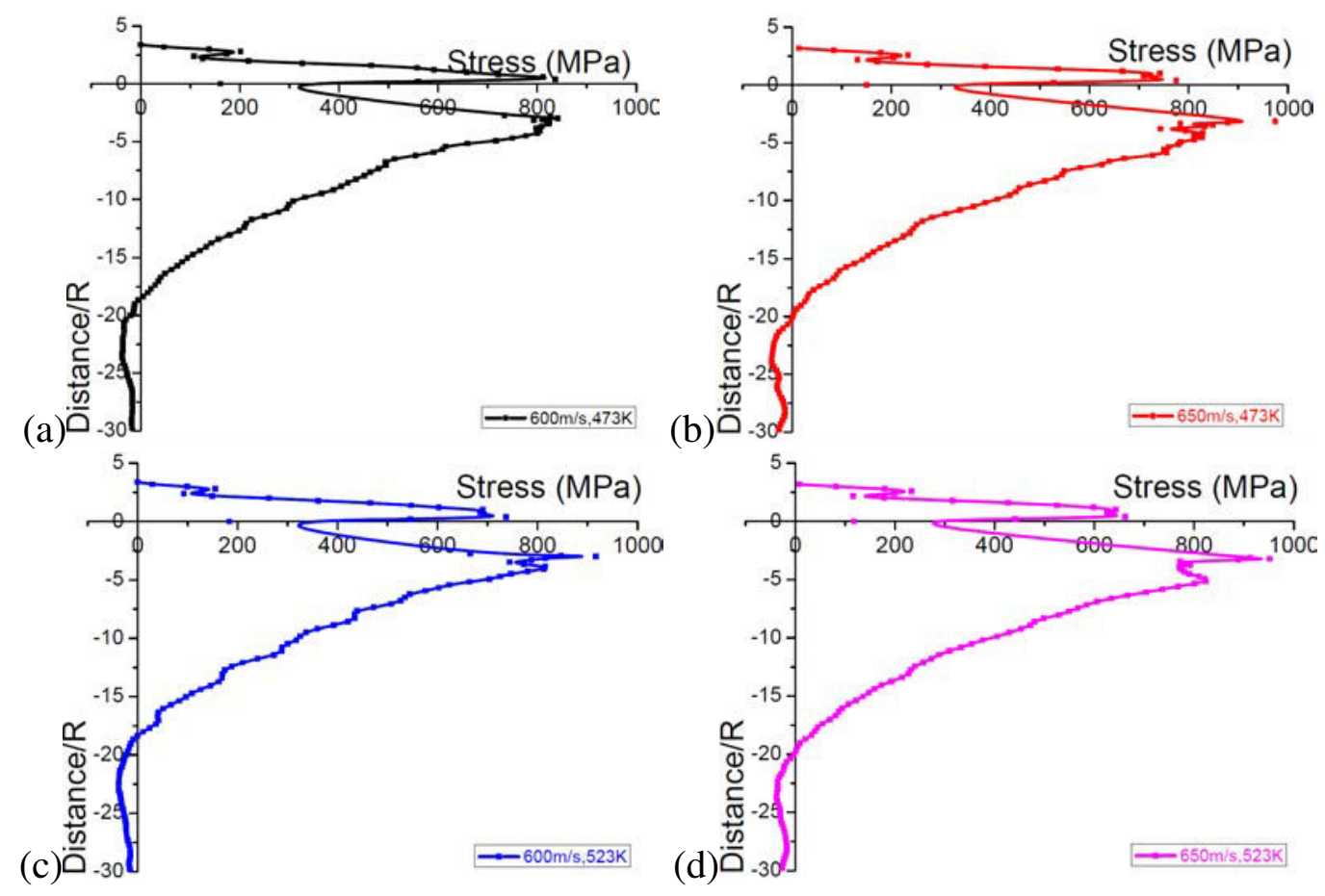

Figure 4.13: Simulation results of the through thickness mean stress distributions for $\mathrm{Cu} / \mathrm{Al}$. (a) case $4-600 \mathrm{~m} / \mathrm{s}, 473 \mathrm{~K}$; (b) case $5-650 \mathrm{~m} / \mathrm{s}, 473 \mathrm{~K}$; (c) case 6 - $600 \mathrm{~m} / \mathrm{s}$, $523 \mathrm{~K}$; (d) case $7-650 \mathrm{~m} / \mathrm{s}, 523 \mathrm{~K}$. 


\section{Chapter 5}

\section{Simulation of erosion}

The normal impact behaviors of particles have been discussed in the previous chapters. In the last chapter of this thesis, therefore, we should advance our study into the oblique impact of particles, since the contact angle is another key parameter of cold spray technology which affects the deposition efficiency of coating. Moreover, tangential sliding of particle due to the bow shock of supersonic gas flow could be the origin of erosion of the substrate surface. The dynamic failure model is first introduced in this study, in order to study the surface erosion of substrate. The effects of contact angle, impact velocity and friction coefficient on the erosion rate are then explored. Further discussion about the propagation of crack provides a better and complete understanding of this oblique impact problem.

\section{Contents}

5.1 Simulation outline $\ldots \ldots \ldots \ldots \ldots \ldots$

5.1 .1 Dynamic failure model . . . . . . . . . . . . . . 83

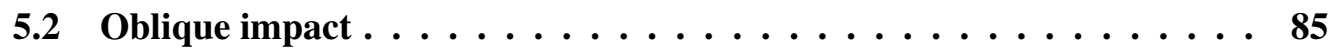

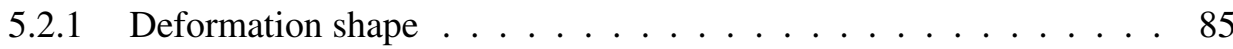

5.2.2 Evolution of the strain and temperature . . . . . . . . . . 86

5.3 Surface erosion . . . . . . . . . . . . . . . . . 87 


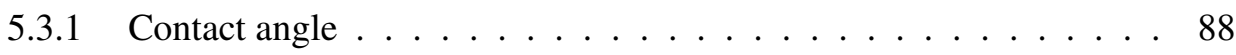

5.3 .2 Velocity . . . . . . . . . . . . . . . 89

5.3 .3 Friction coefficient . . . . . . . . . . . . . . 90

5.3 .4 Multiple impacts . . . . . . . . . . . . . . . . 91

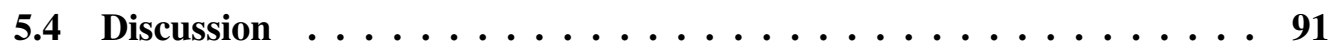

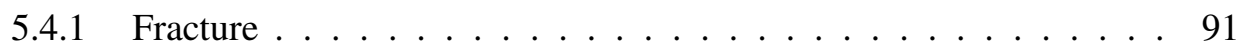

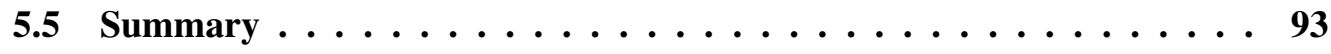




\subsection{Simulation outline}

In this study the contact angle is defined as the angle between the particle velocity vector and the surface of the substrate, as shown in FIG.5.1. Because of the tangential loading, one half model is considered(FIG.5.2). Single impact model, as illustrate in FIG.5.2a, is created for investigating the oblique impact behavior with and without material damage model. ELTOBGY [ELT 05] points out that at least three particles are needed to simulate the erosion process and obtain a stable erosion rate data. Therefore, we build a five particles model, as shown in FIG.5.2b, for the simulation of multiple oblique impacts.

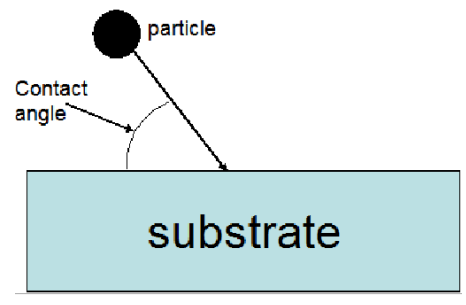

Figure 5.1: Schematic diagram of contact angle.
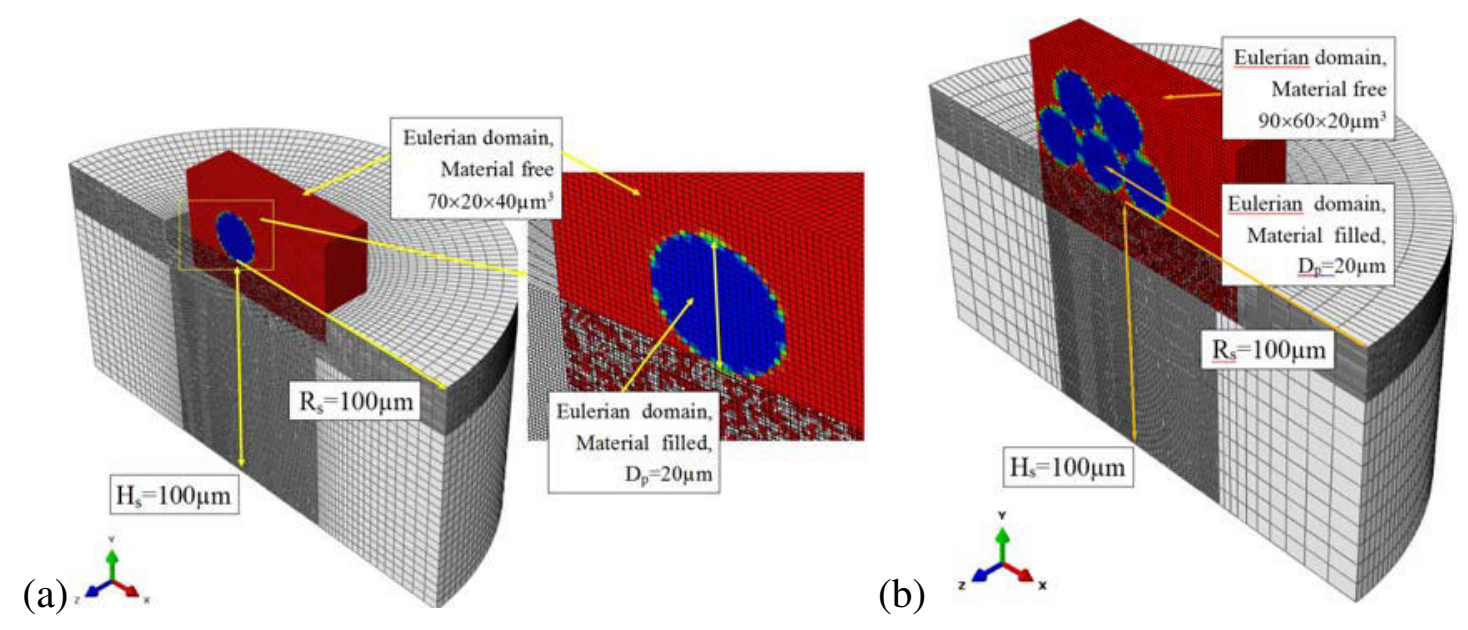

Figure 5.2: Schematic diagrams of (a) one half model and (b) multiple oblique impacts model.

Comparing to the former chapter, the mesh structure and element type are the same. ZSYMM symmetry boundary condition is applied to the X-Y plane of substrate, and the bottom of substrate has no freedom. General contact allows the use of element-based surfaces to model surface erosion for analyses. If an appropriate 'interior' surface is defined, the surface topology will evolve to match the exterior of elements that have not failed. The method about selecting the eroding surfaces is discussed in detail in Appendix H. TAB.5.1 gives the brief outline of the processing conditions for simulation of single particle impact (SP) and multiple particles impacts (MP) in this chapter, focusing on the parameters of contact angle, impact velocity and friction coefficient. 


\begin{tabular}{|c|c|c|c|c|c|}
\hline Case & Materials & Damage & Contact angle $\left(^{\circ}\right)$ & Velocity $(m / s)$ & Friction \\
\hline SP1 & $\mathrm{Cu} / \mathrm{Cu}$ & No & $30-50-70$ & 500 & 0.3 \\
SP2 & $\mathrm{Cu} / \mathrm{Cu}$ & Yes & $10-30-50-70-90$ & 500 & 0.3 \\
SP3 & $\mathrm{Cu} / \mathrm{Cu}$ & Yes & 50 & $400-500-600$ & 0.3 \\
SP4 & $\mathrm{Cu} / \mathrm{Cu}$ & Yes & 50 & 500 & $0.1-0.3-0.5$ \\
MP1 & $\mathrm{Cu} / \mathrm{Cu}$ & Yes & 50 & 500 & 0.3 \\
\hline
\end{tabular}

Table 5.1: Calculation plan for the simulation of oblique impact.

A path along the horizontal direction of substrate surface named path 3 (FIG.5.3) is defined in order to observe simulation results, including equivalent plastic strain and temperature.

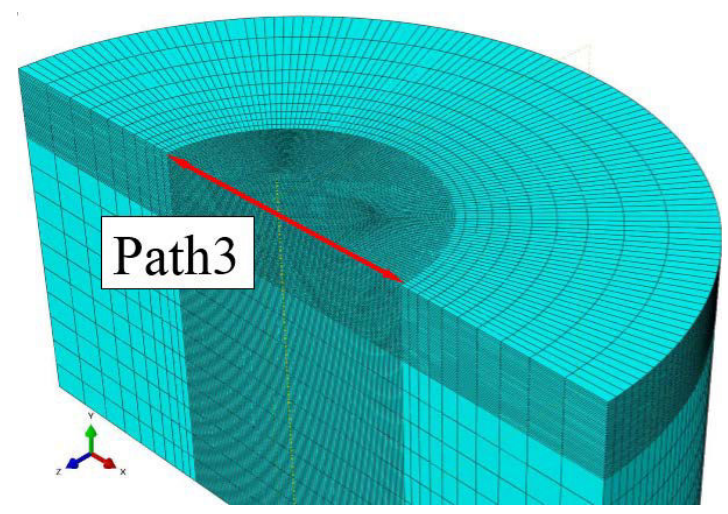

Figure 5.3: Schematic diagram of path 3.

\subsubsection{Dynamic failure model}

The material damage model is introduced for simulating the surface erosion. ABAQUS/Explicit offers two element failure models suitable for high-strain-rate dynamic problems. One is shear failure model driven by plastic yielding, and the other one is tensile failure model driven by tensile loading.

\subsubsection{Shear failure model}

The shear failure model is based on the value of the equivalent plastic strain of element integration points; failure is assumed to occur when the damage parameter exceeds 1 . The damage parameter, $\omega$, is defined as:

$$
\omega=\frac{\bar{\varepsilon}_{0}^{p l}+\sum \Delta \bar{\varepsilon}^{p l}}{\bar{\varepsilon}_{f}^{p l}}
$$


Where $\bar{\varepsilon}_{0}^{p l}$ is any initial value of the equivalent plastic strain, $\Delta \bar{\varepsilon}^{p l}$ is an increment of the equivalent plastic strain, $\bar{\varepsilon}_{f}^{p l}$ is the strain at failure, and the summation is performed over all increments in the analysis.

The strain at failure, $\bar{\varepsilon}_{f}^{p l}$, is assumed to depend on the plastic strain rate, $\dot{\bar{\varepsilon}}^{p l}$; a dimensionless pressure-deviatoric stress ratio, $p / q$ (where $p$ is the pressure stress and $q$ is the Mises stress); and the nondimensional temperature, $\hat{\theta}$, defined earlier in the Johnson-Cook hardening model; and predefined field variables.

The strain at failure is of the form:

$$
\bar{\varepsilon}_{f}^{p l}=\left[d_{1}+d_{2} \exp \left(d_{3} \frac{p}{q}\right)\right]\left[1+d_{4} \ln \left(\frac{\dot{\bar{\varepsilon}}^{p l}}{\dot{\varepsilon}_{0}}\right)\right]\left(1+d_{5} \hat{\theta}\right)
$$

Where $d_{1}-d_{5}$ are failure parameters measured at or below the transition temperature $\theta_{\text {transition }}$, and $\dot{\varepsilon}_{0}$ is the reference strain rate. The values of $d_{1}-d_{5}$ [YIL 11] are given in TAB .5.2. This expression for $\bar{\varepsilon}_{f}^{p l}$ differs from the original formula published by Johnson and Cook [ $\mathrm{JOH} \mathrm{85]} \mathrm{in} \mathrm{the} \mathrm{sign} \mathrm{of} \mathrm{the} \mathrm{parameter} d_{3}$. This difference is motivated by the fact that most materials experience an increase in $\bar{\varepsilon}_{f}^{p l}$ with increasing pressure-deviatoric stress ratio; therefore, $d_{3}$ in the above expression takes positive value.

\subsubsection{Tensile failure model}

The ABAQUS/Explicit tensile failure model uses the hydrostatic pressure stress as a failure measure. The tensile failure criterion assumes that failure occurs when the pressure stress, $p$, becomes more tensile than the user-specified hydrostatic cutoff stress, $\sigma_{\text {cutoff }}$. In our current study $\sigma_{\text {cutoff }}=\sigma_{t}$, the tensile strength of $\mathrm{Cu}$ is shown in TAB.5.2. When the tensile failure criterion is met at an integration point, all the stress components will be set to zero and that material point fails. By default, if all of the material points at any one section of an element fail, the element is removed from the mesh.

\subsection{Oblique impact}

\subsubsection{Deformation shape}

Before proceeding the investigation into the surface erosion, let us first discuss the typical material behavior under oblique impact without considering the material damage. The deformation behaviors of $\mathrm{Cu}$ particle at different contact angles obtained from the experiments [LI 10b] and modeled by the CEL numerical approach are shown in FIG.5.4 and FIG.5.5, respectively.

The deformed shapes obtained by using the CEL numerical approach agree well with the experiment results. The particles present an asymmetrical deformation. A larger tangential displacement of the particle along the substrate surface is observed. The deformation of particle is concentrated on one side at the leading edge of the particle. Owing to the presence of a tangential component of the contact force, a rotational movement of 


\begin{tabular}{|c|c|}
\hline Properties(Unit) & Copper \\
\hline Density, $\rho\left(\mathrm{kg} / \mathrm{m}^{3}\right)$ & $8.9 \times 10^{3}$ \\
\hline Shear Modulus, $G(G P a)$ & 44.7 \\
\hline Tensile strength, $\sigma_{t}(\mathrm{MPa})$ & 220 \\
\hline Thermal conductivity, $\lambda(W / m \cdot K)$ & 232 \\
\hline Specific heat, $c(\mathrm{~J} / \mathrm{kg} \cdot \mathrm{K})$ & 383 \\
\hline Sound velocity, $c_{0}(\mathrm{~m} / \mathrm{s})$ & 3940 \\
\hline Slope in $U_{s}$ versus $U_{p}, s$ & 1.489 \\
\hline Grüneisen coefficient, $\Gamma_{0}$ & 2.02 \\
\hline Yield stress, $A(M P a)$ & 90 \\
\hline Hardening constant, $B(M P a)$ & 292 \\
\hline Hardening exponent, $n$ & 0.31 \\
\hline Strain rate constant, $C$ & 0.025 \\
\hline Thermal softening exponent, $m$ & 1.09 \\
\hline Melting temperature, $T_{m}(K)$ & 1356 \\
\hline Reference temperature, $T_{0}(K)$ & 298 \\
\hline $5 *$ Johnson-Cook failure model, $d_{1}$ & 0.54 \\
\hline$d_{2}$ & 4.89 \\
\hline$d_{3}$ & 3.03 \\
\hline$d_{4}$ & 0.014 \\
\hline$d_{5}$ & 1.12 \\
\hline
\end{tabular}

Table 5.2: Material properties of copper used in the simulation.

the particles along an axis perpendicular to the plane of symmetry occurs. At the trailing edge, the interface may be open resulting in a gap due to this rotational movement.

\subsubsection{Evolution of the strain and temperature}

The simulation contours of the equivalent plastic strain and temperature within the substrate for different contact angles after $60 \mathrm{~ns}$ impact are shown in FIG.5.6 and FIG.5.7, respectively. The maximum equivalent plastic strain situates close to the leading edge of contact area for all of the three impact cases. For clarity, the time history of equivalent plastic strain with various contact angles are plotted in FIG.5.7a. For the contact angle of $70^{\circ}$, before reaching its final plateau at about $45 \mathrm{~ns}$, the equivalent plastic strain increases monotonically; for the contact angle of $30^{\circ}$ and $50^{\circ}$, the saturated limit does not occur at the end of calculations. Approaching to the trialing edge of contact area, the minimum equivalent plastic strain occurs, like FIG.5.6a and b shows; this phenomenon is not observed in the $70^{\circ}$ case (FIG.5.6e and f), on the contrary, a smaller plastic deformation region is found at the bottom of crater.

Secondly, for a constant impact velocity of $500 \mathrm{~m} / \mathrm{s}$, as the contact angle decreases, the tangential force increases, the sliding of particle becomes more dominant resulting in the extending of the contact domain along the horizontal direction of the substrate 

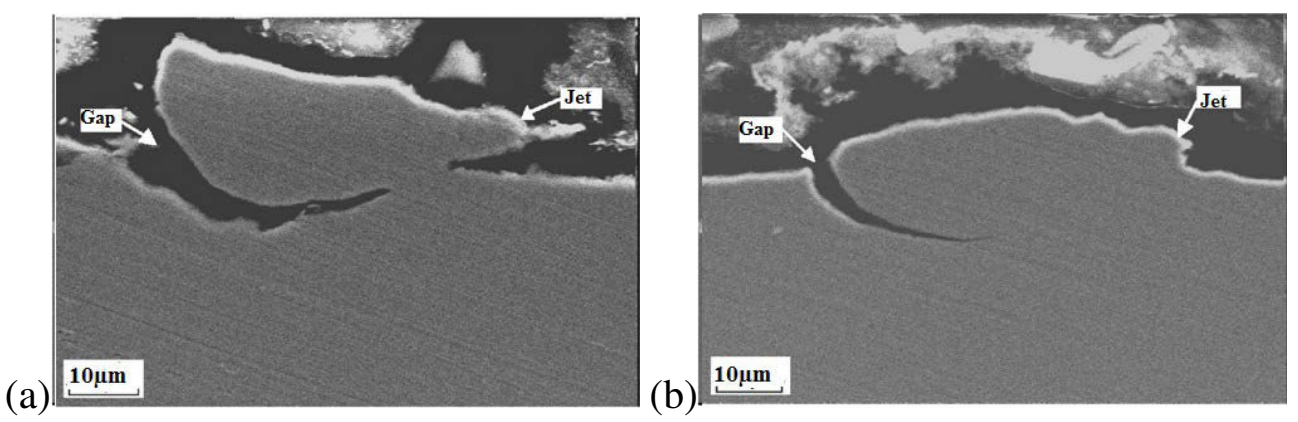

Figure 5.4: SEM images of $\mathrm{Cu} / \mathrm{Cu}$ with contact angle of (a) $50^{\circ}$ and (b) $70^{\circ}$.

(a)

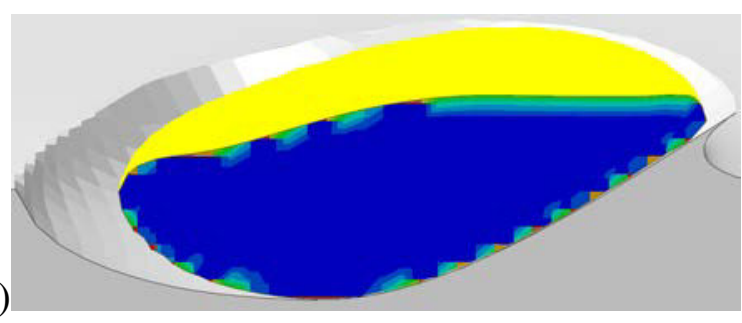

(b)

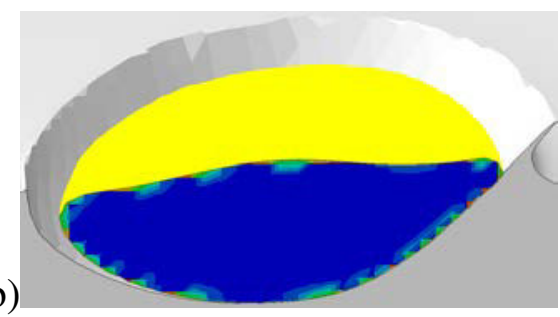

Figure 5.5: Simulation profiles of $\mathrm{Cu} / \mathrm{Cu}$ with contact angle of (a) $50^{\circ}$ and (b) $70^{\circ}$.

surface; on the other hand, the normal component of impact velocity declines, which leads to the shrinking of indentation of crater. The contact area projected onto X-Z plane is found to be more like a half ellipse. The tendency is more significant at a lower contact angle, comparing FIG.5.6b and FIG.5.6f. Additionally, for both the $70^{\circ}$ and $50^{\circ}$ cases, the material jet of substrate is produced at the leading edge of contact area; for $30^{\circ}$ case, however, there is no material jet at the substrate surface.

A higher temperature is gained at the leading edge of contact area, as illustrated in FIG.5.7. $50^{\circ}$ case achieves the highest temperature $500 \mathrm{~K}$ at $31 \mathrm{~ns}$; nevertheless, with a higher contact angle, the heating rate of substrate is faster, which means the $70^{\circ}$ case reaches its maximum temperature firstly, as the blue curve exhibited in FIG.5.8b.

FIG.5.9 and FIG.5.10 show the evolution of the equivalent plastic strain and temperature along the Path 3 on the substrate surface corresponding to various times, respectively. Both the distribution of equivalent plastic strain and temperature are asymmetrical, especially at the lower contact angle of $30^{\circ}$. The tangential impact velocity has a dominant influence on the substrate when the contact angle equals to $30^{\circ}$. The distributions along the negative $\mathrm{X}$-axis is quite few, and stop at the position of $0.5 \mathrm{R}$. However, at higher contact angle of $70^{\circ}$, the plastic deformation region extends over $1 \mathrm{R}$ along the negative $\mathrm{X}$-axis. Moreover, the equivalent plastic strain and temperature distributions of $70^{\circ}$ show two clear peaks unlike the curve shape of $30^{\circ}$, which implies the perpendicular loading becomes prevailing. Although the temperature distribution of $70^{\circ}$ in the initial contact has two maximum value located at the two sides of contact area, only the one located at the positive $\mathrm{X}$-axis remains at the end of the calculation. The longer the contact time, the higher the equivalent plastic strain and the larger the contact area. 


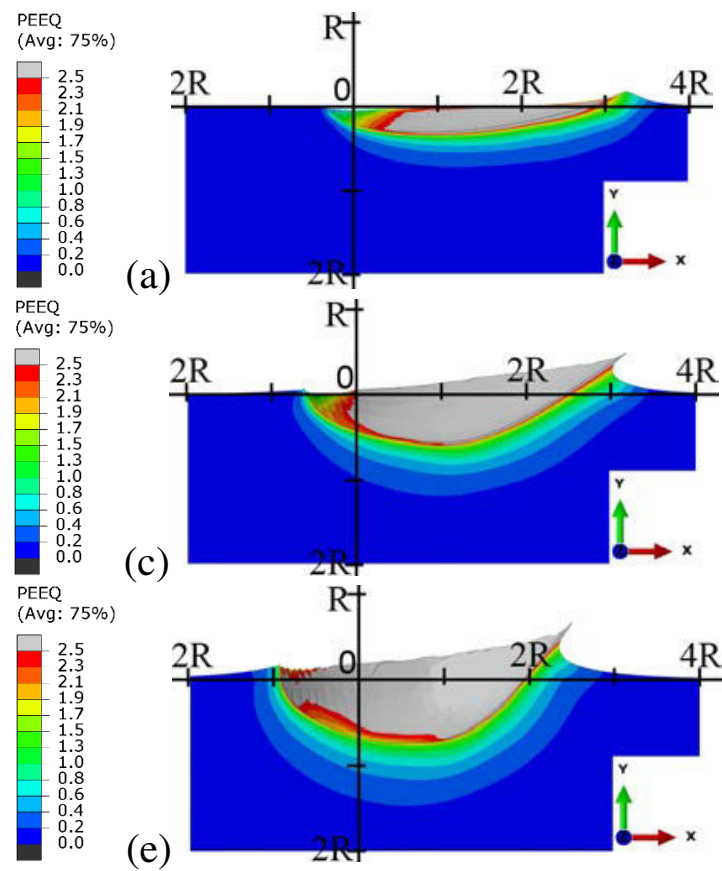

(b)

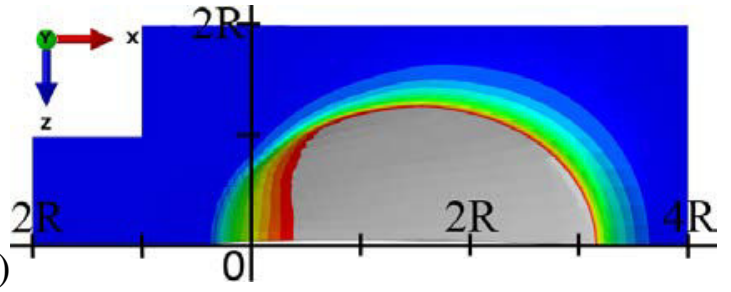

(d)

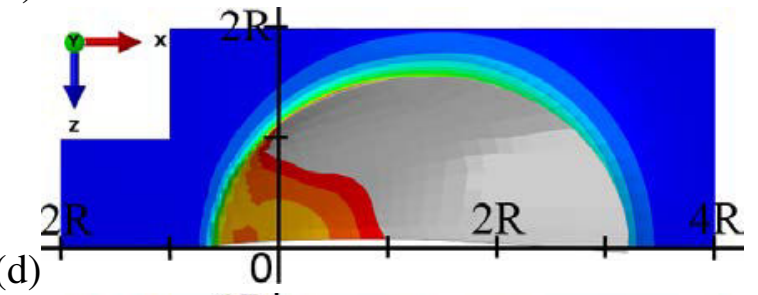

(f)

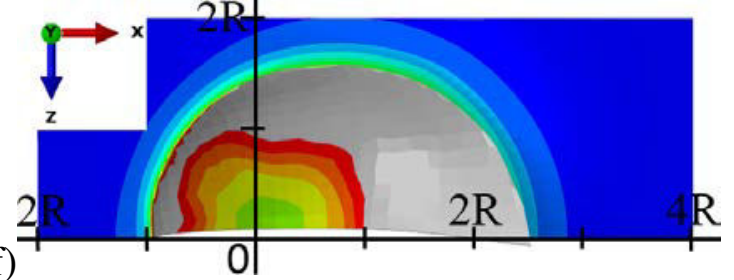

Figure 5.6: The simulation contours of equivalent plastic strain (PEEQ) of $\mathrm{Cu}$ substrate impacted by $\mathrm{Cu}$ particle with contact angle of $30^{\circ}(\mathrm{a}, \mathrm{b}), 50^{\circ}(\mathrm{c}, \mathrm{d})$ and $70^{\circ}(\mathrm{e}, \mathrm{f})$.

The corresponding calculated profiles of the equivalent plastic strain and temperature along the Path 3 are shown in FIG.5.11 with various contact angles. A decrease of the contact angle renders a smaller plastic deformation region between the particle and the substrate. Consequently, the bonding between the particles and the substrate becomes weaker. Overall the temperature at the interface increases when decreasing the contact angle. This can be attributed to the fact that a heat source of friction is created at the contact interface. If one keeps constant the impact velocity it is obvious that a decrease of the impact angle produces both a decrease of the normal component and an increase of the tangential component of the velocity, resulting in creating additional heat by friction. Therefore a decrease of the contact angle results in a local increase of the sliding velocity between the contacting surfaces, thereof increasing the heat generated by friction, see FIG.5.11b.

\subsection{Surface erosion}

The volume erosion rate is defined as a wear volume per unit mass of impacted particles as:

$$
E R_{\text {sim }}=\frac{\text { Vol }_{\text {ero }}}{M_{p s}}
$$

Where the $\mathrm{ER}_{\text {sim }}$ is the volume erosion rate of the particle material $\left(\mathrm{mm}^{3} / \mathrm{g}\right)$, and the 


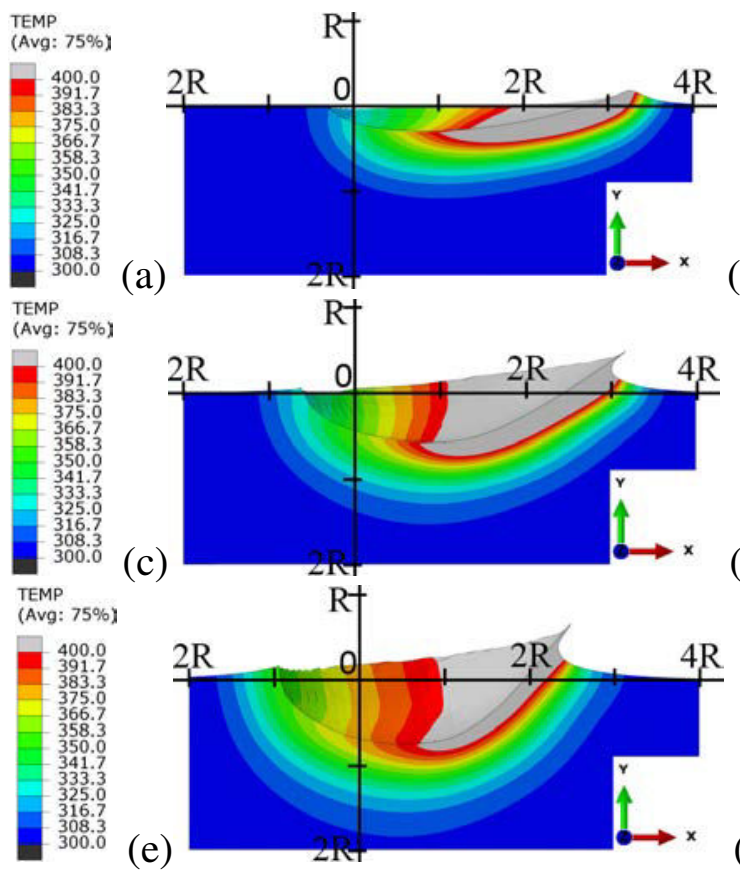

(b)

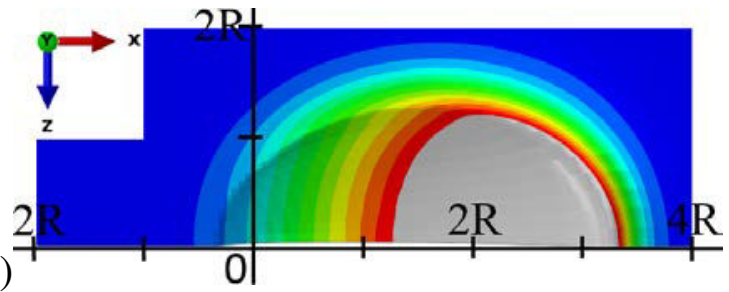

(d)

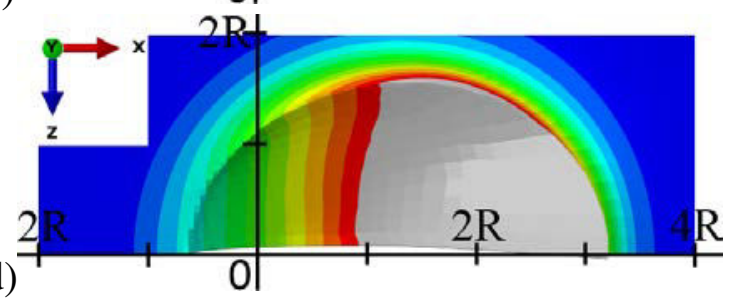

(f)

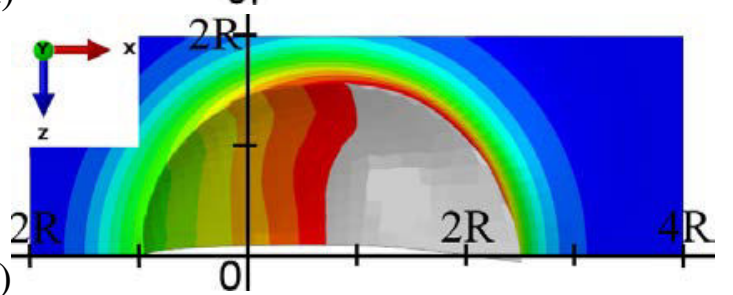

Figure 5.7: The simulation contours of temperature (TEMP) of the $\mathrm{Cu}$ substrate impacted by a $\mathrm{Cu}$ particle with contact angle of $30^{\circ}(\mathrm{a}, \mathrm{b}), 50^{\circ}(\mathrm{c}, \mathrm{d})$ and $70^{\circ}(\mathrm{e}, \mathrm{f})$.

$\mathrm{V}_{\text {ero }}$ and $\mathrm{M}_{\mathrm{ps}}$ stand for the volume of removed elements within the substrate and the total mass of particles, respectively.

As explained in Appendix $\mathrm{H}$, all the elements that may involve in the contact are selected as a element set. Once an element fails, its faces are removed from the contact domain, and any interior faces that have been exposed are activated. A contact edge is removed when all the elements that contain the edge have failed. New contact edges are not created as elements erode.

\subsubsection{Contact angle}

We begin by fixing the impact speed at $500 \mathrm{~m} / \mathrm{s}$ and varying the impact angle from $10^{\circ}$ to $90^{\circ}$. Results shown in FIG.5.12 represent irreversible substrate surface topology versus contact angle. Taking the contact angle of $30^{\circ}$ as an example, the evolution of contact surface of $\mathrm{Cu}$ substrate (as the red rectangular area shown in FIG.5.12a) is illustrated in FIG.5.13. At $36 n s$, a few elements closing to the leading edge of contact domain have been already removed. The equivalent plastic strain of these elements all reach their equivalent plastic strain at failure calculated by the Equation (5.2). Reaching $42 n s$, more and more elements are removed from the substrate surface, and the deformation of the remain elements is larger and severer. The front of the contact domain moves forward along the direction of the tangential velocity component. A fraction of the exterior layer elements is removed as shown in FIG.5.13c, and the faces of the second layer elements of substrate are exposed and activated in the simulation. The majority of contact surface 
(a)

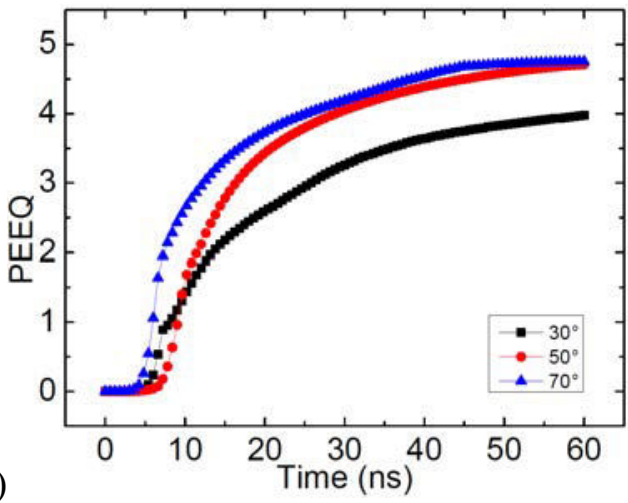

(b)

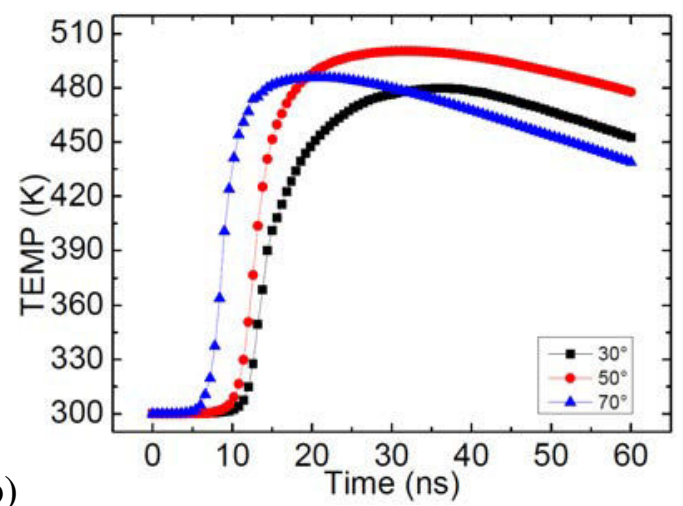

Figure 5.8: Time histories of the (a) equivalent plastic strain (PEEQ) and (b) temperature (TEMP) of the $\mathrm{Cu}$ substrate by using the CEL numerical approach with contact angle of $30^{\circ}, 50^{\circ}$ and $70^{\circ}$.

(a)

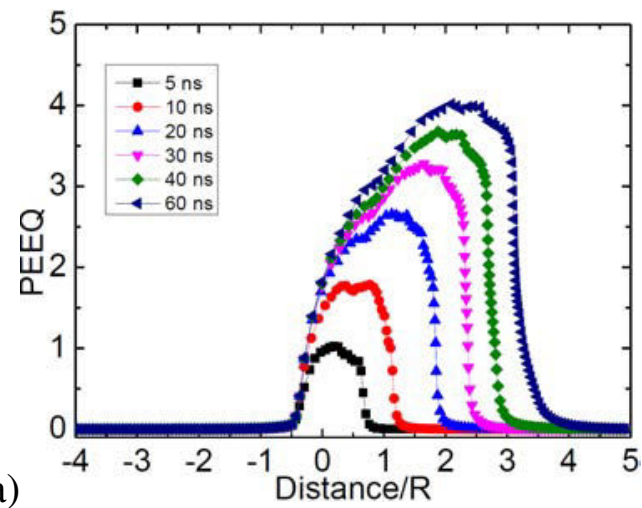

(b)

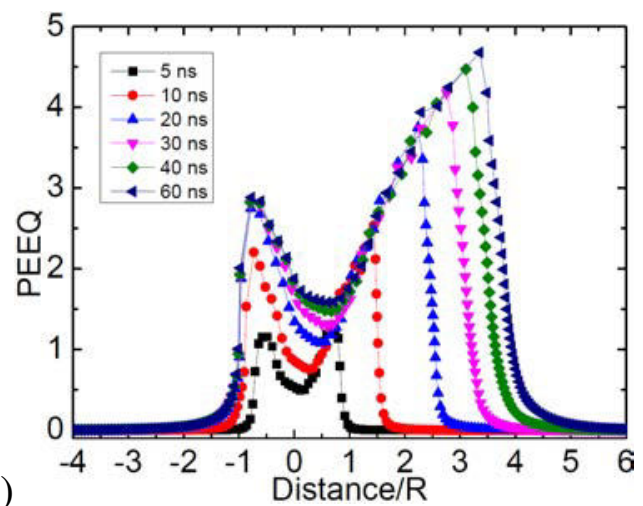

Figure 5.9: Development of the equivalent plastic strain (PEEQ) along the path 3 of the $\mathrm{Cu}$ substrate by using the CEL numerical approach with contact angle of (a) $30^{\circ}$ and (b) $70^{\circ}$.

is eroded till $60 n s$, and the elements have been exposed outside are still involved in the erosion procedure and should be removed as long as they meet the damage initiation criteria. Hence, the elements of substrate surface are removed layer by layer, like the substrate material is eroded piece by piece.

The simulation results show a peak erosion rate at contact angle of around $40^{\circ}$ (FIG.5.14), which is a typical behavior of ductile materials as mentioned in [MOL 02, CHE 03, SHI 99, ELT 05]. The angle at which the peak erosion occur is material dependent. The increasing trend of the penetrate depth shown in FIG.5.14 can be explained by the increasing normal component of the velocity. With higher contact angles, higher normal component of the velocity exists, causing deeper penetration with lower material removal. This has also been observed by SHIMUZU [SHI 01].

FIG.5.15a shows a predicted kinetic energy loss at various contact angles. This result agrees with the results presented in FIG.5.8(a), i.e. the higher the contact angle, the higher the equivalent plastic strain, thus more kinetic energy is lost near the normal contact angle. 
(a)

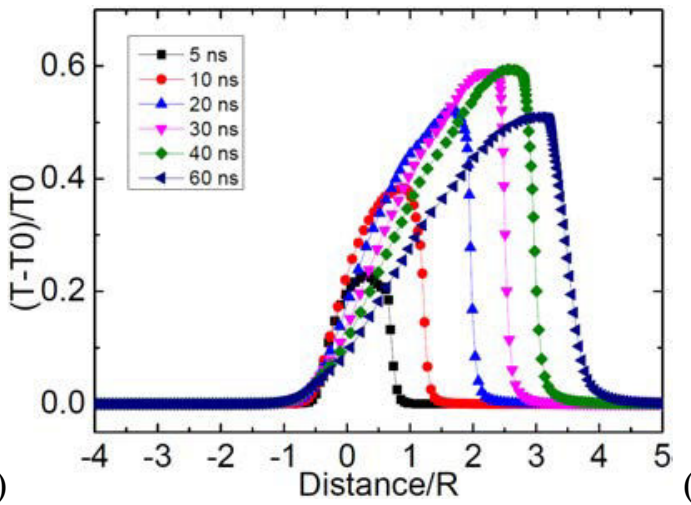

(b)

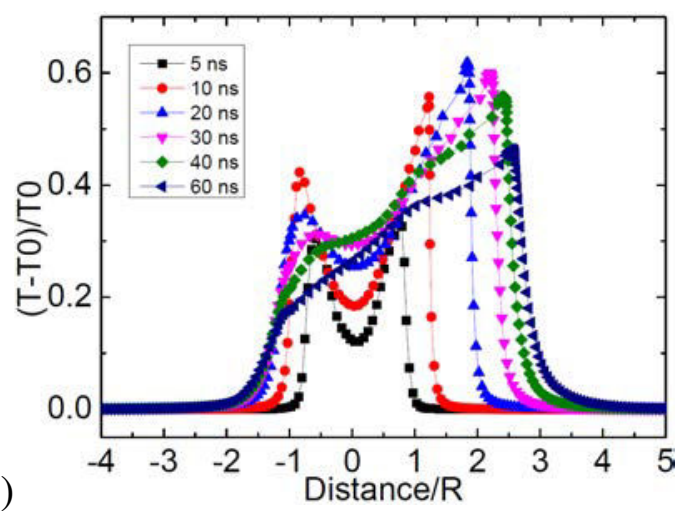

Figure 5.10: Development of the temperature (TEMP) along the path 3 of the $\mathrm{Cu}$ substrate by using the CEL numerical approach with contact angle of (a) $30^{\circ}$ and (b) $70^{\circ}$.

(a)

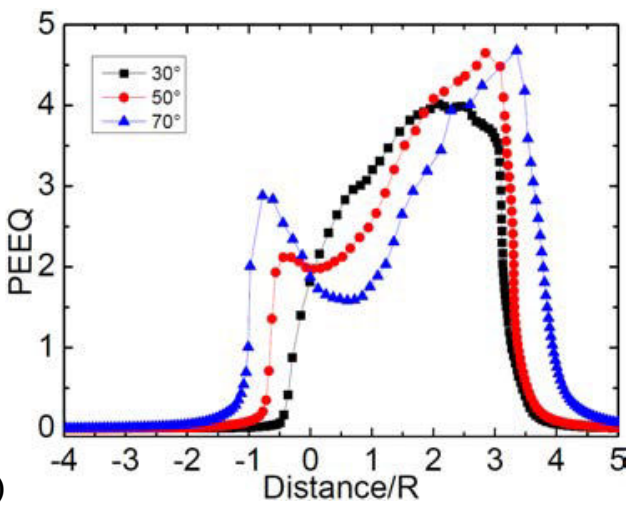

(b)

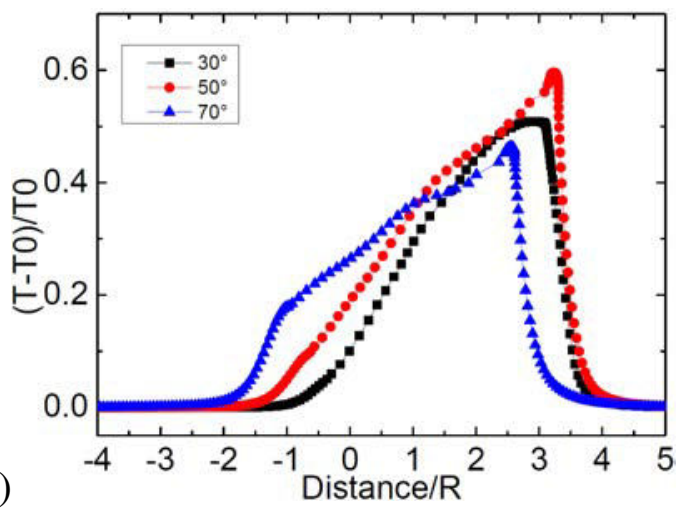

Figure 5.11: Equivalent plastic strain (PEEQ) and temperature (TEMP) along the path 3 of the $\mathrm{Cu}$ substrate by using the CEL numerical approach with contact angle of $30^{\circ}, 50^{\circ}$ and $70^{\circ}$.

FIG.5.15b shows the temperature rise in the substrate at various contact angles. Two main heat sources are considered in the analysis: the heat generated due to the plastic working of the material; and the heat generated as a result of friction. At the contact angle near to $90^{\circ}$, as have discussed in Chapter 3, most of heat is supplied by the plastic deformation of the substrate material, and the friction produces a very limited extent of heat. Conversely, as the contact angle decreases, the sliding velocity and, correspondingly, the effect of friction, increases and the temperature rise becomes steadily higher. Therefore, a maximum rise of temperature must be attained at intermediate angles. Indeed, the computed temperature rise peaks at the contact angle of $50^{\circ}$ as illustrated in FIG.5.15b.

\subsubsection{Velocity}

Next, we fix the contact angle at $50^{\circ}$ and investigate the effect of impact velocity. FIG.5.16 shows the relation between the erosion rate and the impact velocity. Clearly, a higher impact velocity brings about a higher erosion rate. The erosion rate increase with 

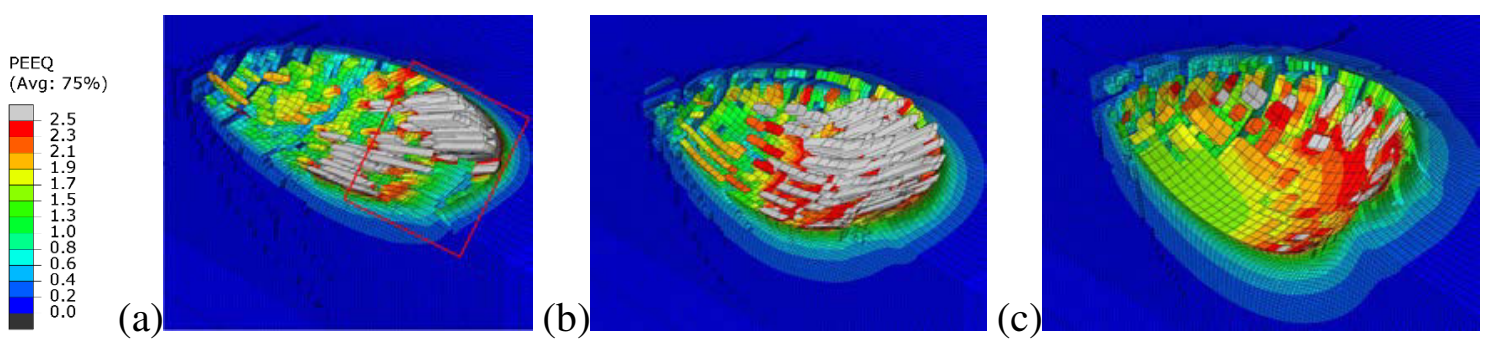

Figure 5.12: Simulation profiles of surface erosion of $\mathrm{Cu}$ substrate impacted by $\mathrm{Cu}$ particle with contact angle of (a) $30^{\circ}$, (b) $50^{\circ}$ and (c) $70^{\circ}$.
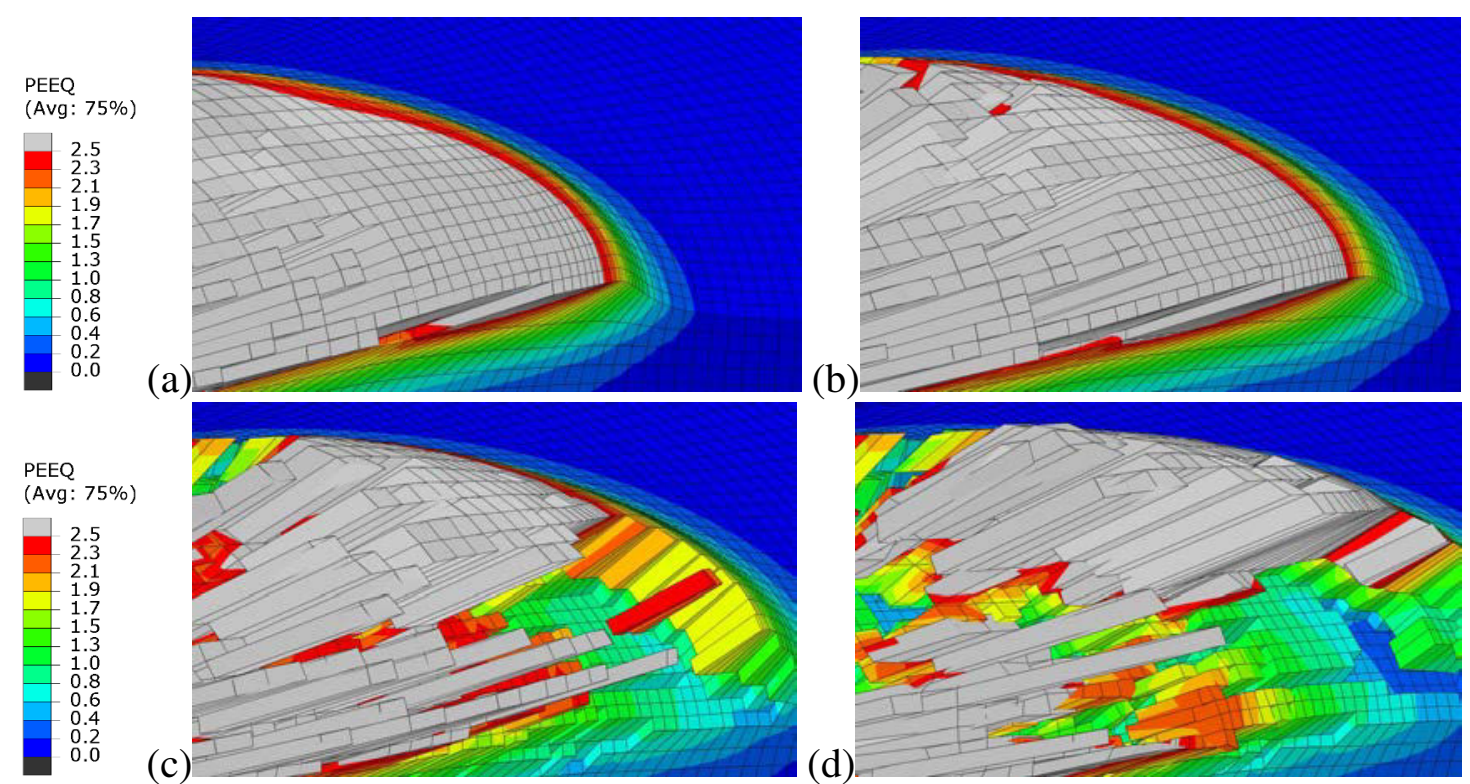

Figure 5.13: The evolution of contact surface of $\mathrm{Cu}$ substrate impacted by a $\mathrm{Cu}$ particle with contact angle of $30^{\circ}$. (a) $36 n s$; (b) $42 n s$; (c) $48 n s$; (c) $60 n s$.

the increase of the impact velocity can be explained by the rise of the kinetic energy of the particles. This leads to more erosion of the material at the same mass of impact particles. Consequently, the indentation is deeper at higher impact velocity as FIG.5.16 displayed.

The erosion rate $E R$ and the impact velocity $V$ has such a relationship [WAN 08, CHE 03]:

$$
E R \propto V^{n}
$$

The relation follows an exponential form with an exponent of 2.04 in this study. RUFF and WIEDERHORN [RUF 79] determined experimentally the velocity exponent to be in the range of $2-3$. The value of the velocity exponent 2 is also present in Finnin's [FIN 60] theories, who conceives erosion as microcutting. Although the finite element simulation does not use any experimentally determined constants, it is able to predict the velocity exponent within the same range. 


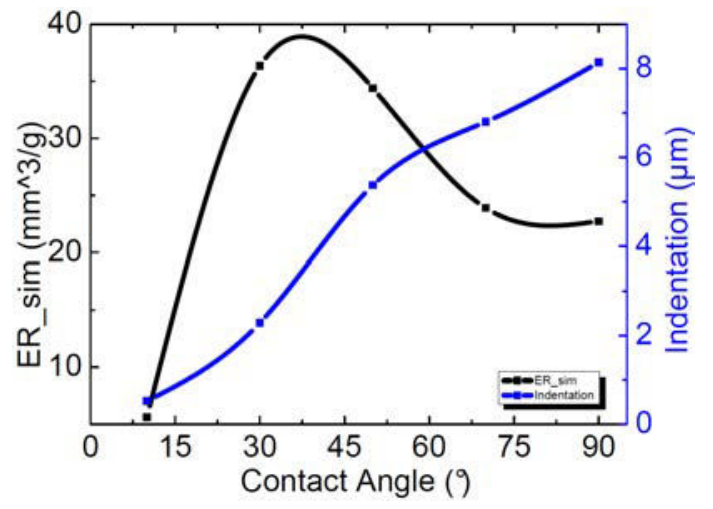

Figure 5.14: Simulation results of erosion rate and indentation versus contact angle.

(a)

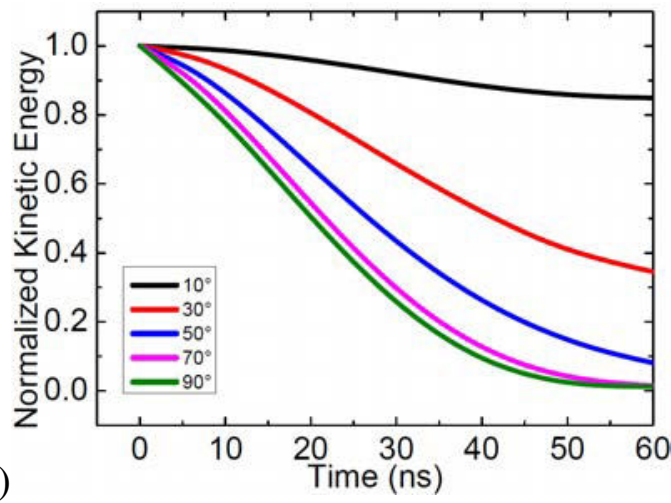

(b)

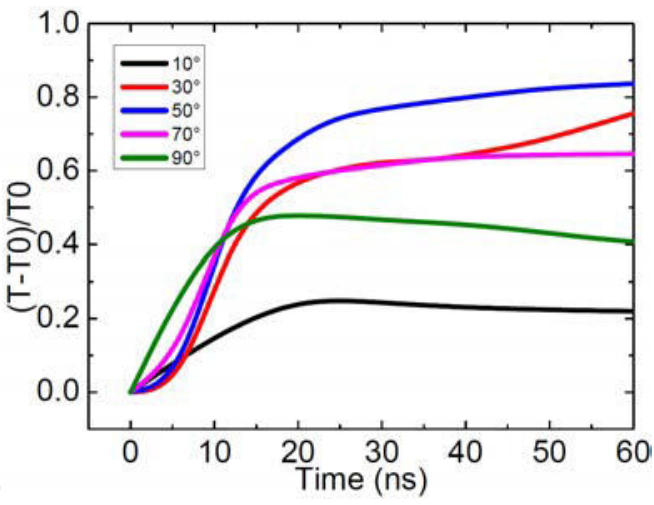

Figure 5.15: Impact of the $\mathrm{Cu}$ substrate by a $\mathrm{Cu}$ particle at $500 \mathrm{~m} / \mathrm{s}$ with various contact angles. (a) Time history of normalized kinetic energy; (b) Time history of normalized temperature rise.

FIG.5.17a shows the predicted kinetic energy loss at various impact velocities. At the fixed contact angle of $50^{\circ}$, the impact velocity has not significant influence on the kinetic energy loss. The critical impact velocity for $\mathrm{Cu} / \mathrm{Cu}$ case is $507 \mathrm{~m} / \mathrm{s}$. When the impact velocity is higher as $600 \mathrm{~m} / \mathrm{s}$, the deformation of substrate happens too rapidly for heat conduction to operate effectively, and the material loses its bearing capacity as a result of thermal softening. According to the localized shear instability theory, the reduction in the flow stress has the effect of limiting the plastic dissipation rate and, consequently, the heating rate [MOL 02, ELT 05]. This trend manifests itself in the declining of the temperature rise at high impact velocities, FIG.5.17b.

\subsubsection{Friction coefficient}

Thirdly, we fix the contact angle at $50^{\circ}$ and the impact velocity at $500 \mathrm{~m} / \mathrm{s}$ and investigate the effect of friction coefficient. The results presented in FIG.5.18 suggest that a higher friction coefficient leads to a higher erosion rate and a deeper crater of substrate. 


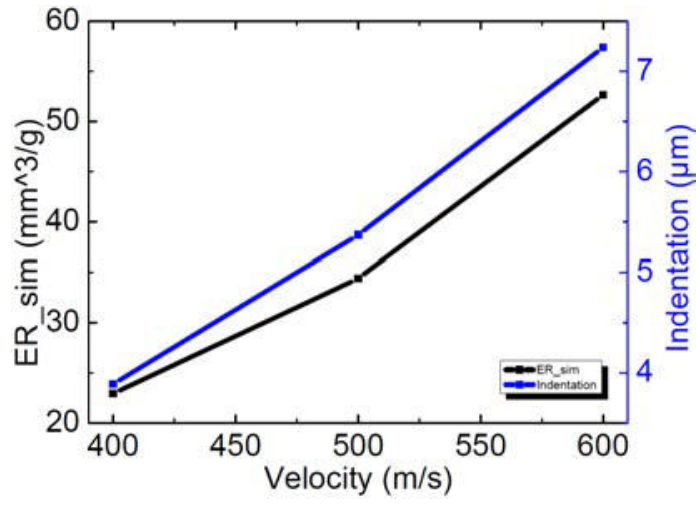

Figure 5.16: Simulation results of erosion rate and indentation versus impact velocity.

(a)

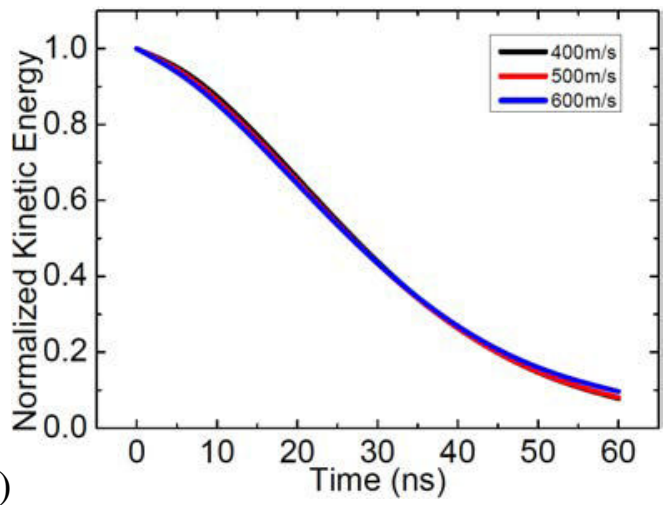

(b)

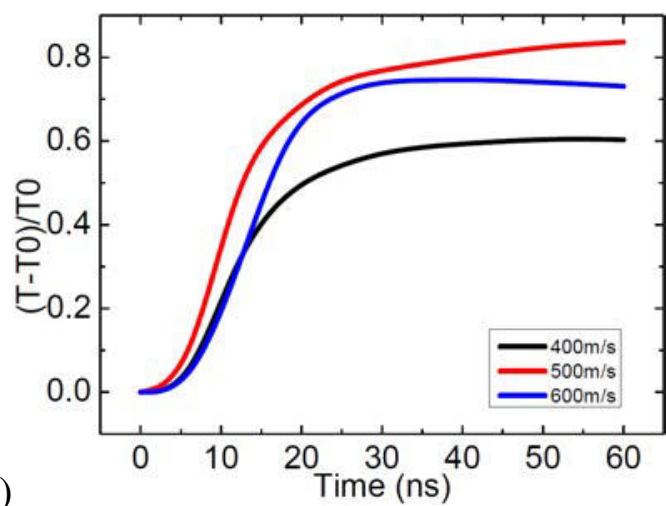

Figure 5.17: Impact of the $\mathrm{Cu}$ substrate by a $\mathrm{Cu}$ particle at various impact velocities with contact angle of $50^{\circ}$. (a) Time history of normalized kinetic energy; (b) Time history of normalized temperature rise.

But the magnitude is obviously lower than that of a lower contact angle or a higher impact velocity, which implies the influence of friction coefficient is limited.

The kinetic energy loss rises about $2 \%$ when the friction coefficient increases from 0.1 to 0.5 as the FIG.5.19a shows. As investigated in the Chapter 3, increasing friction coefficient leads to a sharp decrease of sliding distance, which results in the reducing of friction energy. Consequently, the distribution of friction energy to heat source decreases. That is why the maximum temperature declines when the friction coefficient equals to 0.5 , FIG.5.19b. These observations suggest that, for the $\mathrm{Cu}$ material under consideration, the effective friction coefficient may be a function of sliding velocity. A velocity-dependent friction model should be considered in the further study.

\subsubsection{Multiple impacts}

The simulation results of multiple impacts at impact velocity of $500 \mathrm{~m} / \mathrm{s}$ with contact angle of $50^{\circ}$ are illustrated in FIG.5.20, which depicts the final deformed configurations 


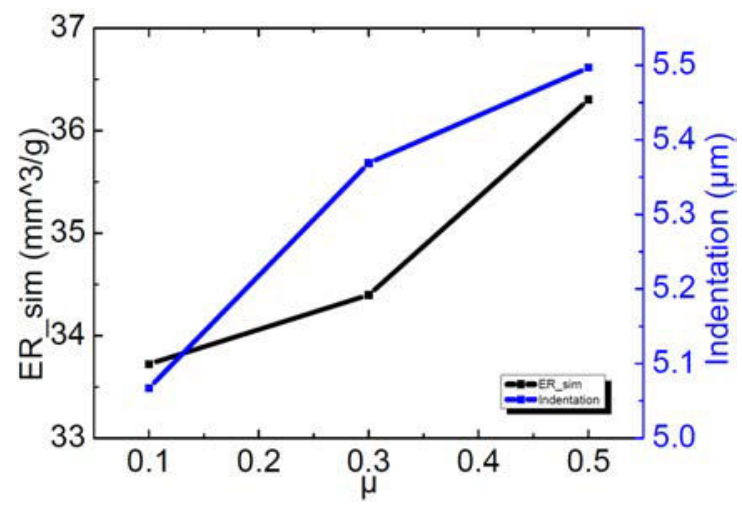

Figure 5.18: Simulation results of erosion rate and indentation versus friction coefficient.
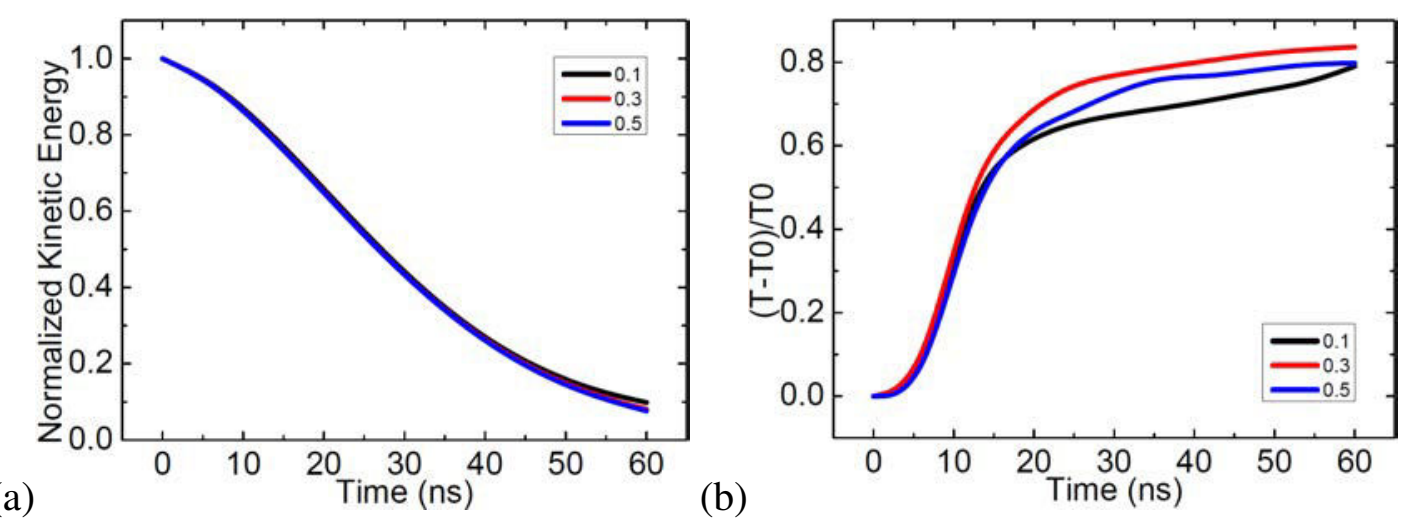

Figure 5.19: Impact of the $\mathrm{Cu}$ substrate by a $\mathrm{Cu}$ particle at $500 \mathrm{~m} / \mathrm{s}$ with various friction coefficient. (a) Time history of normalized kinetic energy; (b) Time history of normalized temperature rise.

of the particles FIG.5.20a, as well as the substrate FIG.5.20b. Both the particles and substrate yield and undergo larger plastic deformations compared to the single impact case, FIG.5.15b. The erosion rate of multiple impacts is $39.153 \mathrm{~mm}^{3} / \mathrm{g}$, which is higher than that of single impact $\left(34.396 \mathrm{~mm}^{3} / \mathrm{g}\right)$, which proves again that a single particle model is not sufficient to simulate the erosion process. Woytowitz [WOY 99], Griffin [GRI 04] and ElTobgy [ELT 05] also give the same conclusion.

Thanks to this multiple impacts model, it is possible to observe the erosion process from a micro perspective. FIG.5.21 illustrates the evolution of contact domain at various times. At $12 n s$, the surface erosion starts near the trailing edge of each crater. Reaching $32 n s$, the surface erosion has extended to the majority of contact domain. Almost all the exterior elements of substrate fail till $76 \mathrm{~ns}$, some of the interior faces have exposed. A whole new contact domain is activated at $100 \mathrm{~ns}$, which means the first layer elements of substrate have been removed all. Based on this algorithm, the active contact domain evolves layer by layer during the analysis as elements fail. 


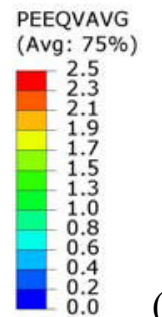

(a)

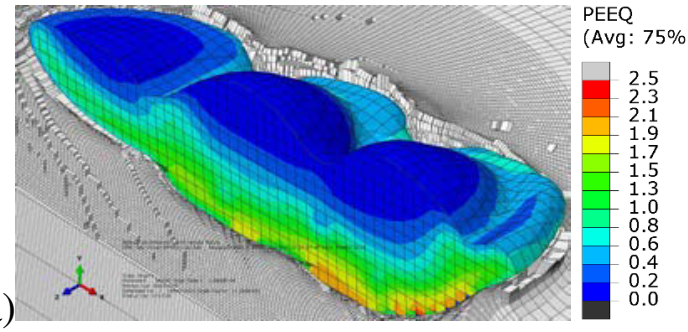

(b)

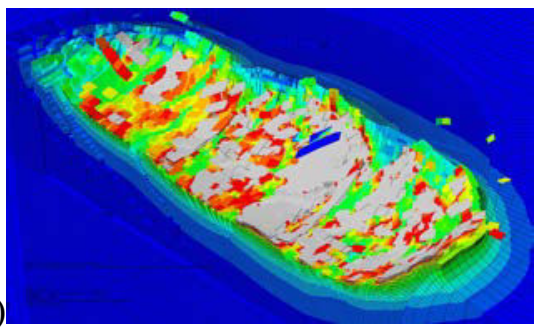

Figure 5.20: Impact of the $\mathrm{Cu}$ substrate by multiple $\mathrm{Cu}$ particle at $500 \mathrm{~m} / \mathrm{s}$ with contact angle of $50^{\circ}$. (a) Deformation shape of particles; (b)Deformation shape of substrate.

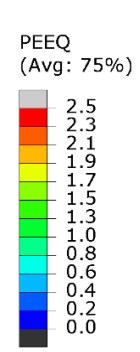

(a)
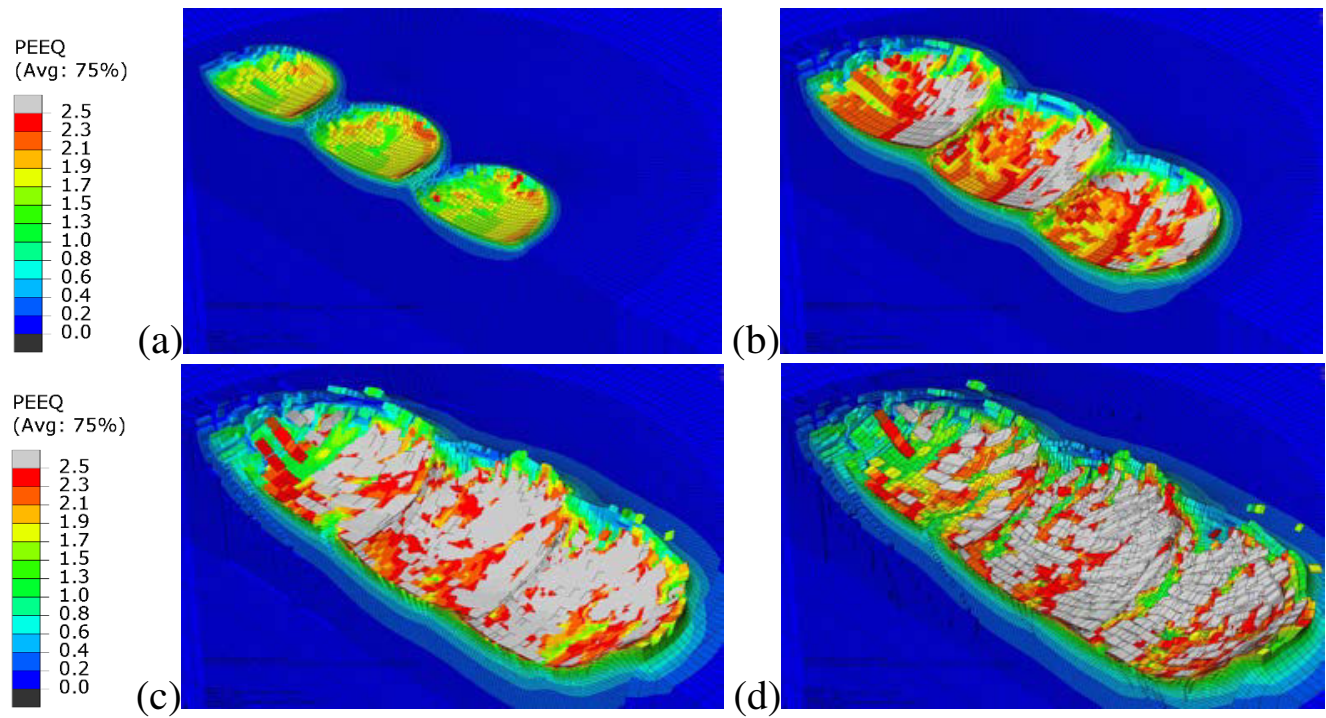

Figure 5.21: The evolution of contact domain of $\mathrm{Cu}$ substrate impacted by multiple $\mathrm{Cu}$ particle at $500 \mathrm{~m} / \mathrm{s}$. (a) $12 \mathrm{~ns}$; (b) $32 \mathrm{~ns}$; (c) $76 \mathrm{~ns}$; (d) $100 \mathrm{~ns}$.

\subsection{Discussion}

\subsubsection{Fracture}

In our study of the surface erosion, there are signs of crack formation below the substrate surface as FIG.5.12 and FIG.5.20 show.

For a ductile material, it has a relatively high resistance to impact due to its good capability to accommodate plastic deformation. It is known that the fracture is generally caused by tensile or shear stress. FIG.5.22 gives schematic diagrams of these two fracture modes. The elements with tensile failure mode mainly distribute around the craters of substrate and under the substrate surface; the elements with shear failure mode, nevertheless, appear more at the contact domain of substrate surface.

It is of importance to investigate how these cracks start and grow in understanding the erosion process and mechanism involved. FIG.5.23 depicts the onset and propagation of cracks. Basically, it contains four steps: void formation, FIG.5.23a; crack formation 


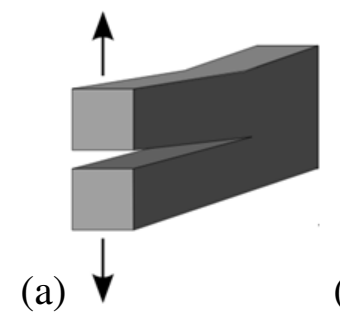

(b)

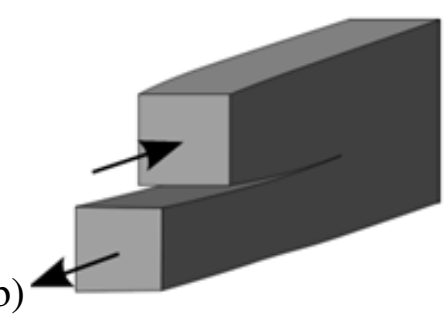

Figure 5.22: The schematic diagrams of (a) tensile failure mode and (b) shear failure mode.

by coalescence of cavities, FIG.5.23b; crack growth, FIG.5.23c; coalescence cracks and erosion by wear particle removal, FIG.5.23d.

Voids are first formed at the trialing edge of the contact domain (the left crater in the figures), which grow outward from the periphery of the crater of substrate. Propagation of horizontal and vertical crack is observed as illustrated in FIG.5.23b. For the contact angle of $50^{\circ}$, the crack propagating in the vertical direction is much quicker than that in the horizontal direction, due to the increase in tensile stress component perpendicular to the cracking direction. With the continuation of oblique load, the propagation of the cracks that are asymmetric about the $\mathrm{Y}$ axis will be enhanced in the subsurface, FIG.5.23c. Some of these cracks may decompose and grow into lateral cracks which propagate approximately parallel to the surface. On the other hand, some of these cracks may merge (FIG.5.23d) and extend further, resulting in the substrate material delamination. Meanwhile, new cracks are formed around the periphery of the other two craters of substrate (the center and the right ones).

According to our simulation results, for a normal impact, at $265 \mathrm{~m} / \mathrm{s}$, the void occurs at the periphery of crater of $\mathrm{Cu}$ substrate. Then we try to find out the damage initiation under different contact angles. We obtain a simple relationship between critical velocity and contact angle as:

$$
v_{\text {ero }}=v_{\pi / 2} \sin (\theta)
$$

Where the $v_{\text {ero }}$ and $v_{\pi / 2}$ stand for the critical velocity under any contact angle $\theta$ and $\pi / 2$, respectively. For normal impact, the $v_{e r o}$ equals to $v_{\pi / 2}$, which for $\mathrm{Cu}$ material is $265 \mathrm{~m} / \mathrm{s}$ in our study, and this $v_{\pi / 2}$ is a function of material, impact velocity, temperature, powder size and so on. For the oblique impact, the critical velocity for activating the surface erosion is lower. Taking contact angle of $30^{\circ}$ as an example, it only needs 130 $\mathrm{m} / \mathrm{s}$ to initiate the formation of void. The fracture we study now is mainly driven by plastic yielding, therefore, another parameter could be used for identifying the damage initiation is the equivalent plastic strain (PEEQ). As long as the PEEQ reaches 1.042, the erosion process starts.

All the discussion presented above is the preliminary investigation on the fracture, the simulation results arouse our interest on the fracture mechanism behind the surface erosion. I have to admit, however, there are still lots of key questions unsolved. Although the element deletion method is remarkably easy to simulation fracture problems within 
(a)

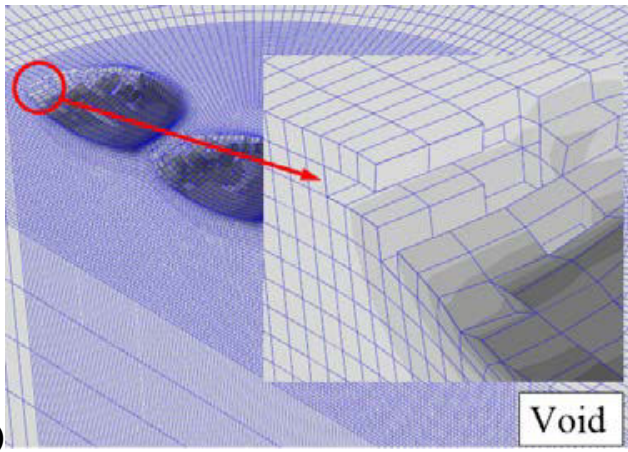

(c)

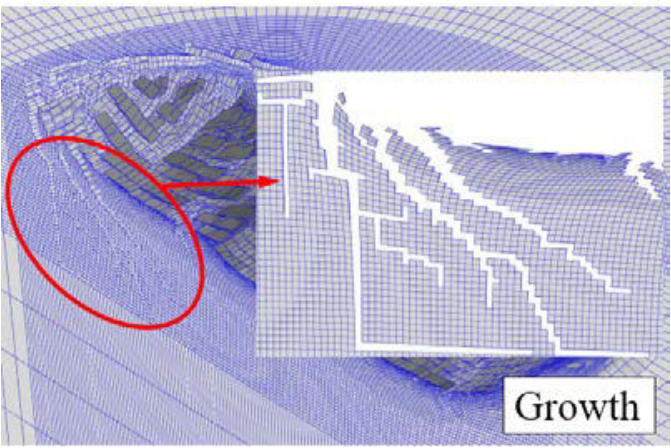

(b)

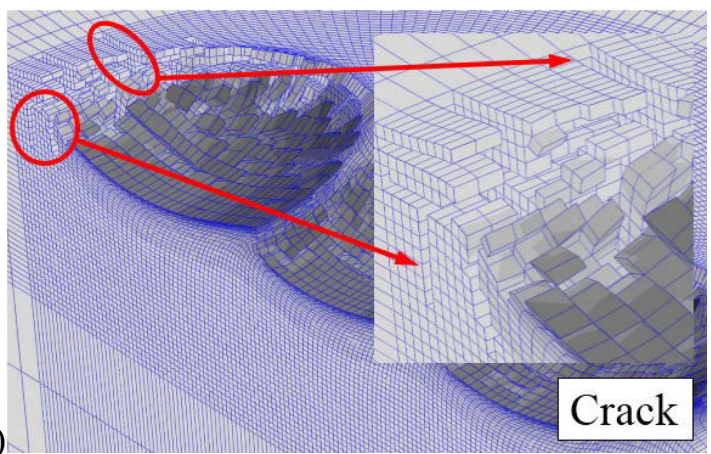

(d)

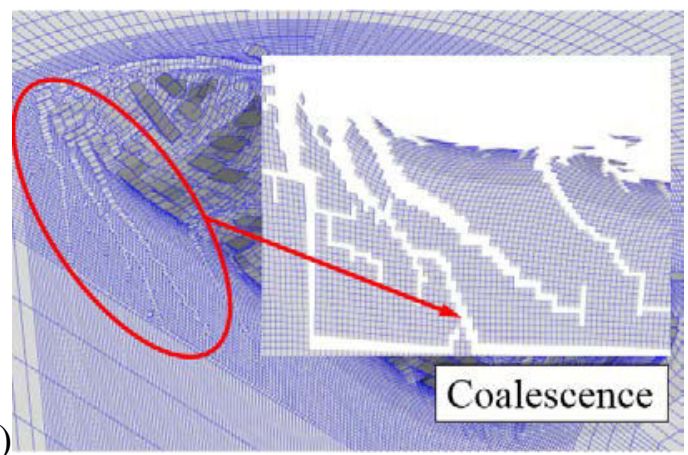

Figure 5.23: The crack propagation within $\mathrm{Cu}$ substrate impacted by multiple $\mathrm{Cu}$ particles at $500 \mathrm{~m} / \mathrm{s}$. (a) $12 \mathrm{~ns}$; (b) $32 \mathrm{~ns}$; (c) $76 \mathrm{~ns}$; (d) $100 \mathrm{~ns}$.

the classical FEM framework, however, as energy is lost when elements are deleted, it is imperative to adjust the constitutive law with respect to the element size lest spurious mesh dependence of the fracture energy be introduced [BEL 13]. Moreover, the element deletion method is heavily mesh-dependent. The quality of this method needs to be evaluate by comparing the simulation results and experimental data. In order to weaken the influence from the mesh dependency, ABAQUS/Explicit offers another general capability for modeling progressive damage and failure in ductile metals. The progressive damage models allow for a smooth degradation of the material stiffness, which makes them suitable for both quasi-static and dynamic situations. The preliminary results are presented in Appendix I.

Crack initiation and propagation are affected by a lot of parameters besides impact velocity and contact angle, what are their influences? It is possible to derive a more accurate and reasonable equation than the Equation (5.5) based on those results. $\mathrm{Cu}$ is one of the ductile material, but what will happen for the brittle material under high velocity oblique impact? How and under what condition do the cracks start and grow? How to describe and identify the dynamic fracture of substrate material? What is the influence of the eroded surface on the deposition process and coating properties? It opens a brand new door for us to study the cold spray, and it will help us to understand the deposition process and bonding mechanism more completely. 


\subsection{Summary}

We have performed finite element simulations of the oblique impact consisting of $\mathrm{Cu}$ particles striking $\mathrm{Cu}$ substrate. Damage accumulation is computed by using both shear and tensile failure models, which dynamically remove elements from the finite element mesh as they fail. The range of impact angles investigated varies from $10^{\circ}$ to $90^{\circ}$, and the impact velocities range from 400 to $600 \mathrm{~m} / \mathrm{s}$, and the friction coefficient changes from 0.1 to 0.5 . The CEL numerical approach has the potential to be further expanded both spatially and temporally for investigating the issues of deformation and crack formation, and to consider the effects of impact velocity and contact angle.

Crack formation and material removal could be simulated, and erosion rates were calculated under different considerations. A lower contact angle, a higher impact velocity and a higher friction coefficient all contribute to a higher erosion rate and a deeper indentation of substrate. For a ductile material as $\mathrm{Cu}$, it followed two fracture modes in our study, which are tensile failure mode and shear failure mode. The former one occurred mainly beneath the substrate surface and nearby the periphery of the craters. The latter one was found on the contact domain of substrate surface.

A preliminary investigation on the propagation of crack was given in the last section. Four steps were found during the propagation of cracks: void formation; crack formation by coalescence of cavities; crack growth; coalescence cracks and erosion by wear particle removal. A simple criterion equation was derived based on the simulation results for predicting the initiation of damage under different contact angles. The equivalent plastic strain could also be a parameter for identifying the onset of damage, which is 1.042 for $\mathrm{Cu}$ in our study. There are still lots of meaningful issues worth to discuss in the future. 


\section{Conclusions}

In this chapter, conclusions related to the deformation behaviors of particles and substrate during normal and oblique impacts are drawn based on the finite element analysis. The main achievements during this study are highlighted followed by a discussion of the limitations and perspectives for future work.

\section{CEL}

The cold spray particle deposition process was simulated by modeling the impact of spherical particles onto a flat substrate under various conditions. The deposition process could be described as a high velocity impact problem, thus how to simulate large deformation behaviors of material was our first problem. We, for the first time, proposed the Couple Eulerian Lagrangian (CEL) numerical approach to solve the high strain rate deformation problem. The capability of the CEL numerical approach in modeling the Cold Spray deposition process was verified through a parametric study, including impact velocity, initial temperature of the particle, friction coefficient and materials. The simulation results by using the CEL numerical approach agree with the experimental results published in the literature. Comparing with other numerical approaches discussed in Chapter 2, which are Lagrangian, ALE and SPH, the CEL analyses are generally more accurate and more robust in higher deformation regimes. Even in the material jet area, where the materials undergo extremely high strain rate plastic deformation, the mesh does not result in distortions. The drawback is the extra computational work.

\section{Multiple impacts}

The primary goal of simulation of particle deposition process is to understand the bonding mechanism between the particles and substrate by observing and analyzing the material behaviors during the very short contact time, which is impossible to accomplish in the experiment. Lots of research mentioned in Chapter 1 has performed this simulation by using single particle impact model. We also did it, and the results were presented in Chapter 2, Chapter 3 and one section of Chapter 5. The more important point is the extension to multiple impacts. Inspiring by the concept of crystal structure, we built a FCC-like particles arrangement model. It could be used to investigate the porosity rate 
and residual stress of deposited particles under various conditions. We observed not only the 3D geometry of voids, but also their distributions and developments in time. The compressive residual stresses at the interface between the particles and the substrate is mainly caused by the large and fast plastic deformation. Another comprehensive multiple impact model is created in Chapter 5 for the simulation of surface erosion.

\section{Parameters}

The parameters considered in this study are materials, impact velocity, temperature and friction coefficient. Material is one of the most important factors that affect the deposition process. We could not say that dissimilar combination is absolutely better than similar ones, or the softer substrate is definitely better than harder one. The choice of material depends on the application of coating, service life, machining cost and may be environment-friendly concerns. Once the material is chosen, the successful deposition could be obtained by optimizing the impact velocity and the initial temperature of particles. ТАВ.C. 1 presented below schematically demonstrates the effects of these parameters on the outputs we analyzed in this study, including Porosity rate, Residual stress and Erosion rate. The temperature mentioned in this study is the initial temperature of particles just before impact. How the particles are heated or cold down in the gas jet is not considered.

\begin{tabular}{|c|c|c|c|c|c|}
\hline Parameters & PEEQ & $\Delta$ TEMP & PorosityRate & ResidualStress & ErosionRate \\
\hline$\uparrow$ Velocity & $\uparrow \uparrow$ & $\uparrow \uparrow$ & $\downarrow \downarrow$ & $\uparrow \uparrow$ & $\uparrow \uparrow$ \\
$\uparrow$ Temperature & $\uparrow \uparrow$ & $\uparrow$ & $\downarrow$ & $\uparrow$ & N/A \\
$\uparrow$ Friction & $\uparrow$ & $\rightarrow$ & N/A & N/A & $\uparrow$ \\
$\uparrow$ Angle & $\uparrow$ & $\uparrow \downarrow$ & N/A & N/A & $\downarrow \downarrow$ \\
\hline
\end{tabular}

Table C.1: Schematic illustration of effects of parameters.

\section{Damage}

The last contribution is the introduction of a dynamic failure model in order to study the surface erosion of substrate induced by low angle impacts. ABAQUS/Explicit provides two element failure models suitable for high-strain-rate dynamic problems. The first one is shear failure model driven by Johnson-Cook plastic yielding; the second one is called tensile failure, for which the material fails when the mean stress reaches the ultimate tensile strength. The modeling of oblique multiple impacts has the potential to investigate the issues of deformation and crack formation both spatially and temporally, and to consider the effects of impact velocity, contact angle and friction coefficient. A preliminary investigation on the propagation of the crack was given in the last section of Chapter 5. For a ductile material such as $\mathrm{Cu}$, it follows two fracture modes in our study, 
which are tensile failure mode and shear failure mode. The former one mainly occurred beneath the substrate surface and the periphery of substrate craters, nevertheless the latter one was found predominately at the surface of substrate. We observed four steps during the propagation of cracks: void formation; crack formation; crack growth; coalescence and failure. A simple criterion equation was derived based on the simulation results for predicting the initiation of damage, where the critical erosion velocity $v_{\text {ero }}$ is a function of the contact angle $\theta$ and normal impact velocity $v_{\pi / 2}$. The critical equivalent plastic strain could also be a parameter for identifying the onset of damage, identified as being 1.042 for $\mathrm{Cu}$ in our study.

\section{Perspectives}

Several topics are worthy of consideration for future work.

Based on the current model, we may complete our parametric study. The effect of carrier gas temperature - on porosity rate, residual stress and erosion rate - could be considered either.

How adhesion forces between the particles and the substrate, or between particles themselves, is a key point that should require more attention. It could be used forward to estimate the critical velocity for particle deposition. A velocity-dependent friction model should be considered in further study as well.

This study has considered impacts between ideally spherical particles and the substrate. In fact, most particles used in cold spray are angular. The effect of the shape should play a role on the porosity rate, mostly for hard particles. The effect of particle size distribution should be also worthwhile to investigate.

Regarding the simulation of erosion, how and under what conditions do cracks initiate and grow remains an important question.

The work presented in this thesis is basically a phenomenal study, and we could deepen out view into nanoscale and explain the material behavior from the perspectives of physics and material science. It will definitely enhance our understanding of high velocity impact problem. 


\section{Appendix A: Significance of the substrate size in simulations of the normal impact of a sphere with a half-space.}

\section{Introduction}

In Finite Element (FE) simulations of a sphere with a half-space, on one hand, the substrate size should be large enough to eliminate the constrains of boundary conditions; on the other hand, it should be as economical as possible to save the computational cost. The stress wave propagation is an unavoidable phenomenon during the impact. Therefore, it is important to choose an appropriate substrate that not only the boundary conditions but also the stress waves has a negligible effect on the impact/rebound behaviors.

The analysis model for the normal impact of a sphere with a substrate is shown in FIG.2.2. Three different sizes are chosen for the substrate: $L=2 R, L=10 R$ and $L=20 R$, where $R=12.5 \mu \mathrm{m}$ is specified to the radius of the sphere. The sphere is discretised with the meshes shown in FIG.2.6. The material properties for the sphere and the substrate are shown in TAB.2.1. The initial impact velocity of $840 \mathrm{~m} / \mathrm{s}$ is considered due to it's the highest velocity employed in this study. The modeling procedures are discussed in Section 2.4.1.

\section{The influence of substrate size}

The simulation results, including the displacement, velocity, temperature and kinetic energy, are shown in FIG.AA.1 for the impact of a $25 \mu \mathrm{m}$ Aluminum particle with an Aluminum substrate at $840 \mathrm{~m} / \mathrm{s}$. It can been seen that all results obtained with the substrate of size $10 R \times 10 R$ and $20 R \times 20 R$ are identical, whereas the results obtained with the substrate size of $2 R \times 2 R$ is different from that obtained with the other two substrates. This indicates that a substrate of size $10 R \times 10 R$ is larger enough to eliminate the influence of the boundary constrains and to represent a half-space for the velocity at least up to 840 $m / s$. 
Appendix A: Significance of the substrate size in simulations of the normal impact of a sphere with a half-space.

At high velocities, the stress waves initiated by the impact could affect the responses during the impact. As for material properties selected, the stress wave velocity $(c=$ $\left.(E / \rho)^{1 / 2}\right)$ is about $5000 \mathrm{~m} / \mathrm{s}$, thus the the time for the longitudinal wave to reflect back to the initial contact point in the substrate of size $L=2 R$ is $T_{2 R}=5 n s$, and in the substrate of size $L=10 R$ is $T_{10 R}=50 \mathrm{~ns}$. The impact duration for the substrate size of $L=2 R$ is 52 $n s$, and for the substrate size of $L=10 R$ is $30 n s$. Note that the impact duration of impact is determined by evaluating the instant when the contact pressure reduces to zero. It can be seen that the longitudinal wave could reflect back to the initial contact points 10 times with a substrate of $L=2 R$ at $840 \mathrm{~m} / \mathrm{s}$. This reflection not only provokes a longer contact, but also a rise of amplitude of deformation. The substrate of $L=2 R$ could no longer represent a semi-infinite space. However, for the impact with a substrate of $L=10 R$, at the impact velocity considered, there is no longitudinal wave reflects back to the initial contact point during the impact duration time. We could conclude that the rebound of particle is not due to the reflection of stress wave, and the substrate of $L=10 R$ is large enough to ignore the influence of stress wave.
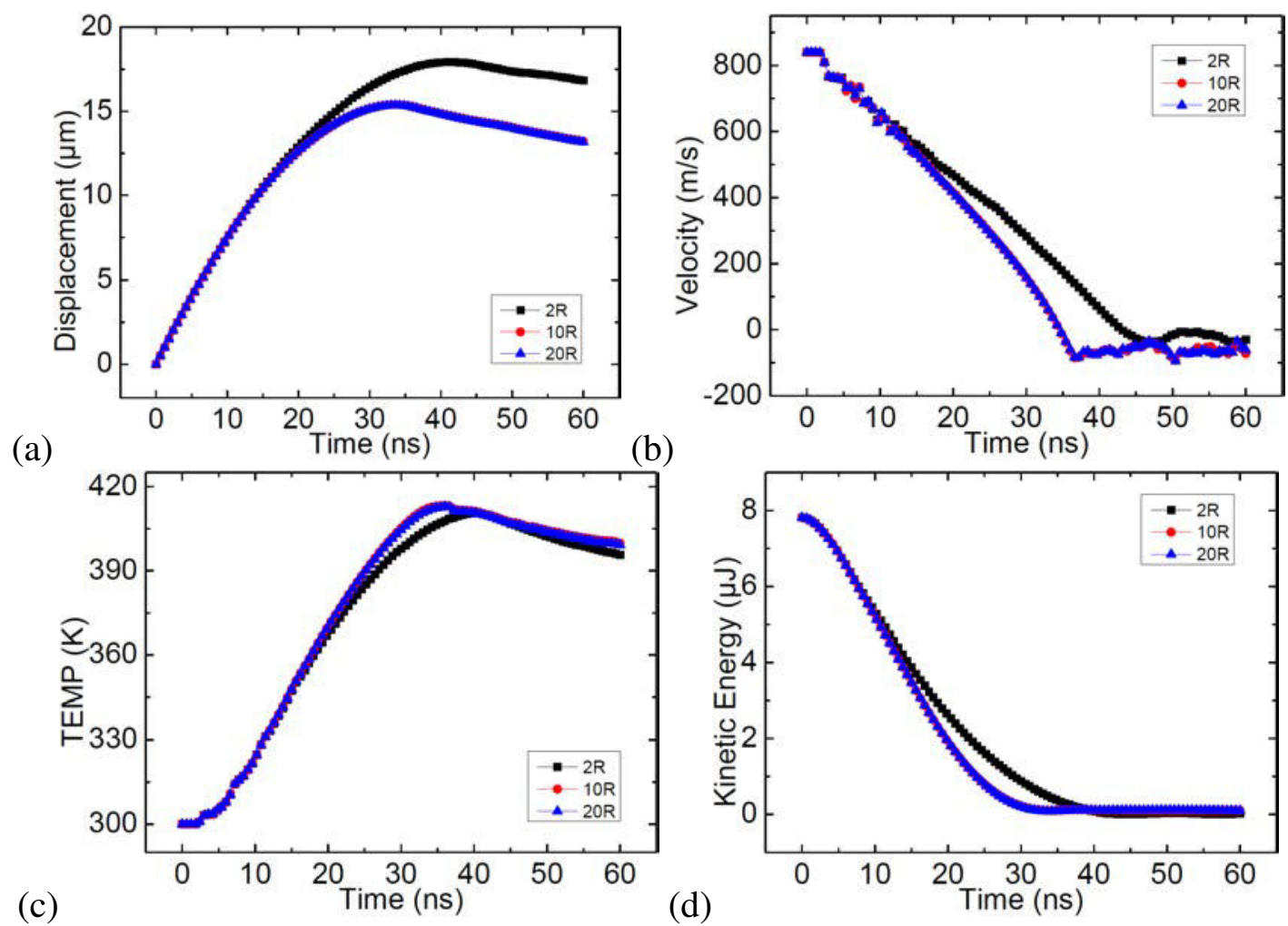

(a)
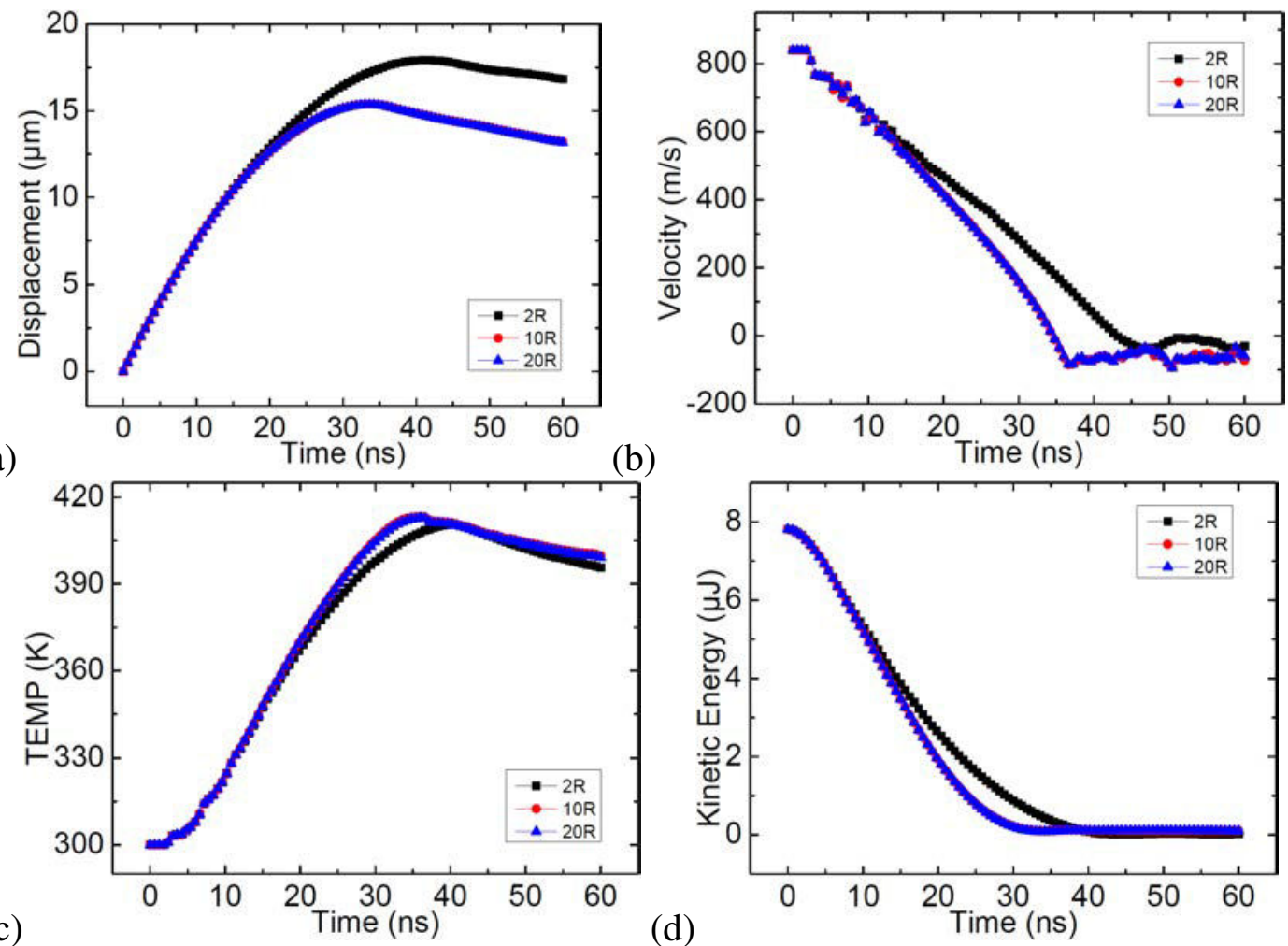

Figure AA.1: Time histories of (a) displacement at the particle center, (b) velocity at the particle center, (c) temperature at the particle center and (d) kinetic energy of particle. 
Appendix A: Significance of the substrate size in simulations of the normal impact of a sphere with a half-space.

\section{Summary}

In this appendix, the significance of substrate size in FE simulations of the normal impact of a sphere with a half-space has been investigated. It has been shown that two of factors need to be considered when constructing the FE models: one is the effect of boundary constrains, which should be so insignificant that the substrate can actually represent a semi-infinite space; the other is the effect of stress wave which is unavoidable in physical impacts, which should exclude its influence on the rebound behavior of particle. Therefore it is suggested that, for all the impact velocities considered in this study, the substrate of size $L=10 R$ is large enough to represent a semi-infinite space. 
Appendix A: Significance of the substrate size in simulations of the normal impact of a sphere with a half-space. 


\section{Appendix B: The temperature-dependent material property for Aluminum and Copper}

\section{Thermal conductivity of Aluminum}

Thermal conductivity $(W / m \cdot K)$, Temperature

$0.000 \mathrm{e}+000,0.0$

$2.3529 \mathrm{e}+004,10.0$

$1.1672 \mathrm{e}+004,20.0$

$4.8952 \mathrm{e}+003,30.0$

$2.3648 \mathrm{e}+003,40.0$

$1.2843 \mathrm{e}+003,50.0$

$7.8583 \mathrm{e}+002,60.0$

$5.1870 \mathrm{e}+002,70.0$

$3.6889 \mathrm{e}+002,80.0$

$3.2384 \mathrm{e}+002,90.0$

$3.0085 \mathrm{e}+002,100.0$

$2.8282 \mathrm{e}+002,110.0$

$2.6898 \mathrm{e}+002,120.0$

$2.5863 \mathrm{e}+002,130.0$

$2.5112 \mathrm{e}+002,140.0$

$2.4587 \mathrm{e}+002,150.0$

$2.4237 \mathrm{e}+002,160.0$

$2.4015 \mathrm{e}+002,170.0$

$2.3883 \mathrm{e}+002,180.0$

$2.3808 \mathrm{e}+002,190.0$

$2.3764 \mathrm{e}+002,200.0$

$2.3731 \mathrm{e}+002,210.0$

$2.3695 \mathrm{e}+002,220.0$

$2.3649 \mathrm{e}+002,230.0$
$2.3592 \mathrm{e}+002,240.0$

$2.3530 \mathrm{e}+002,250.0$

$2.3474 \mathrm{e}+002,260.0$

$2.3443 \mathrm{e}+002,270.0$

$2.3460 \mathrm{e}+002,280.0$

$2.3558 \mathrm{e}+002,290.0$

$2.3720 \mathrm{e}+002,300.0$

$2.3800 \mathrm{e}+002,310.0$

$2.3864 \mathrm{e}+002,320.0$

$2.3915 \mathrm{e}+002,330.0$

$2.3954 \mathrm{e}+002,340.0$

$2.3982 \mathrm{e}+002,350.0$

$2.3999 \mathrm{e}+002,360.0$

$2.4007 \mathrm{e}+002,370.0$

$2.4007 \mathrm{e}+002,380.0$

$2.4000 \mathrm{e}+002,390.0$

$2.3986 \mathrm{e}+002,400.0$

$2.3966 \mathrm{e}+002,410.0$

$2.3941 \mathrm{e}+002,420.0$

$2.3912 \mathrm{e}+002,430.0$

$2.3879 \mathrm{e}+002,440.0$

$2.3842 \mathrm{e}+002,450.0$

$2.3802 \mathrm{e}+002,460.0$

$2.3760 \mathrm{e}+002,470.0$
$2.3715 \mathrm{e}+002,480.0$

$2.3669 \mathrm{e}+002,490.0$

$2.3621 \mathrm{e}+002,500.0$

$2.3571 \mathrm{e}+002,510.0$

$2.3521 \mathrm{e}+002,520.0$

$2.3469 \mathrm{e}+002,530.0$

$2.3416 \mathrm{e}+002,540.0$

$2.3363 \mathrm{e}+002,550.0$

$2.3309 \mathrm{e}+002,560.0$

$2.3254 \mathrm{e}+002,570.0$

$2.3199 \mathrm{e}+002,580.0$

$2.3143 \mathrm{e}+002,590.0$

$2.3086 \mathrm{e}+002,600.0$

$2.3029 \mathrm{e}+002,610.0$

$2.2971 \mathrm{e}+002,620.0$

$2.2912 \mathrm{e}+002,630.0$

$2.2853 \mathrm{e}+002,640.0$

$2.2793 \mathrm{e}+002,650.0$

$2.2731 \mathrm{e}+002,660.0$

$2.2669 \mathrm{e}+002,670.0$

$2.2606 \mathrm{e}+002,680.0$

$2.2541 \mathrm{e}+002,690.0$

$2.2476 \mathrm{e}+002,700.0$

$2.2409 \mathrm{e}+002,710.0$
$2.2341 \mathrm{e}+002,720.0$

$2.2272 \mathrm{e}+002,730.0$

$2.2202 \mathrm{e}+002,740.0$

$2.2130 \mathrm{e}+002,750.0$

$2.2057 \mathrm{e}+002,760.0$

$2.1983 \mathrm{e}+002,770.0$

$2.1908 \mathrm{e}+002,780.0$

$2.1832 \mathrm{e}+002,790.0$

$2.1756 \mathrm{e}+002,800.0$

$2.1678 \mathrm{e}+002,810.0$

$2.1601 \mathrm{e}+002,820.0$

$2.1523 \mathrm{e}+002,830.0$

$2.1445 \mathrm{e}+002,840.0$

$2.1368 \mathrm{e}+002,850.0$

$2.1292 \mathrm{e}+002,860.0$

$2.1217 \mathrm{e}+002,870.0$

$2.1143 \mathrm{e}+002,880.0$

$2.1073 \mathrm{e}+002,890.0$

$2.1004 \mathrm{e}+002,900.0$

$2.0940 \mathrm{e}+002,910.0$

$2.0880 \mathrm{e}+002,920.0$

$2.0824 \mathrm{e}+002,930.0$

$2.0809 \mathrm{e}+002,933.0$ 


\section{Specific heat of Aluminum}

Specific heat $(J / k g \cdot K)$, Temperature

$\begin{array}{llll}4.8166 \mathrm{e}+002,100.0 & 9.1059 \mathrm{e}+002,320.0 & 1.0143 \mathrm{e}+003,540.0 & 1.1221 \mathrm{e}+003,760.0 \\ 5.2824 \mathrm{e}+002,110.0 & 9.1638 \mathrm{e}+002,330.0 & 1.0185 \mathrm{e}+003,550.0 & 1.1285 \mathrm{e}+003,770.0 \\ 5.7060 \mathrm{e}+002,120.0 & 9.2203 \mathrm{e}+002,340.0 & 1.0227 \mathrm{e}+003,560.0 & 1.1350 \mathrm{e}+003,780.0 \\ 6.0903 \mathrm{e}+002,130.0 & 9.2753 \mathrm{e}+002,350.0 & 1.0269 \mathrm{e}+003,570.0 & 1.1418 \mathrm{e}+003,790.0 \\ 6.4382 \mathrm{e}+002,140.0 & 9.3289 \mathrm{e}+002,360.0 & 1.0312 \mathrm{e}+003,580.0 & 1.1487 \mathrm{e}+003,800.0 \\ 6.7525 \mathrm{e}+002,150.0 & 9.3812 \mathrm{e}+002,370.0 & 1.0355 \mathrm{e}+003,590.0 & 1.1558 \mathrm{e}+003,810.0 \\ 7.0358 \mathrm{e}+002,160.0 & 9.4322 \mathrm{e}+002,380.0 & 1.0398 \mathrm{e}+003,600.0 & 1.1632 \mathrm{e}+003,820.0 \\ 7.2907 \mathrm{e}+002,170.0 & 9.4821 \mathrm{e}+002,390.0 & 1.0442 \mathrm{e}+003,610.0 & 1.1708 \mathrm{e}+003,830.0 \\ 7.5198 \mathrm{e}+002,180.0 & 9.5309 \mathrm{e}+002,400.0 & 1.0487 \mathrm{e}+003,620.0 & 1.1786 \mathrm{e}+003,840.0 \\ 7.7251 \mathrm{e}+002,190.0 & 9.5787 \mathrm{e}+002,410.0 & 1.0532 \mathrm{e}+003,630.0 & 1.1867 \mathrm{e}+003,850.0 \\ 7.9091 \mathrm{e}+002,200.0 & 9.6255 \mathrm{e}+002,420.0 & 1.0578 \mathrm{e}+003,640.0 & 1.1950 \mathrm{e}+003,860.0 \\ 8.0738 \mathrm{e}+002,210.0 & 9.6715 \mathrm{e}+002,430.0 & 1.0625 \mathrm{e}+003,650.0 & 1.2036 \mathrm{e}+003,870.0 \\ 8.2213 \mathrm{e}+002,220.0 & 9.7167 \mathrm{e}+002,440.0 & 1.0673 \mathrm{e}+003,660.0 & 1.2124 \mathrm{e}+003,880.0 \\ 8.3533 \mathrm{e}+002,230.0 & 9.7612 \mathrm{e}+002,450.0 & 1.0722 \mathrm{e}+003,670.0 & 1.2216 \mathrm{e}+003,890.0 \\ 8.4717 \mathrm{e}+002,240.0 & 9.8051 \mathrm{e}+002,460.0 & 1.0772 \mathrm{e}+003,680.0 & 1.2310 \mathrm{e}+003,900.0 \\ 8.5782 \mathrm{e}+002,250.0 & 9.8485 \mathrm{e}+002,470.0 & 1.0823 \mathrm{e}+003,690.0 & 1.2407 \mathrm{e}+003,910.0 \\ 8.6743 \mathrm{e}+002,260.0 & 9.8914 \mathrm{e}+002,480.0 & 1.0876 \mathrm{e}+003,700.0 & 1.2506 \mathrm{e}+003,920.0 \\ 8.7614 \mathrm{e}+002,270.0 & 9.9339 \mathrm{e}+002,490.0 & 1.0930 \mathrm{e}+003,710.0 & 1.2609 \mathrm{e}+003,930.0 \\ 8.8410 \mathrm{e}+002,280.0 & 9.9761 \mathrm{e}+002,500.0 & 1.0985 \mathrm{e}+003,720.0 & 1.2641 \mathrm{e}+003,933.0 \\ 8.9141 \mathrm{e}+002,290.0 & 1.0018 \mathrm{e}+003,510.0 & 1.1042 \mathrm{e}+003,730.0 & \\ 8.9820 \mathrm{e}+002,300.0 & 1.0060 \mathrm{e}+003,520.0 & 1.1100 \mathrm{e}+003,740.0 & \\ 9.0457 \mathrm{e}+002,310.0 & 1.0102 \mathrm{e}+003,530.0 & 1.1160 \mathrm{e}+003,750.0 & \end{array}$

\section{Thermal conductivity of Copper}

Thermal conductivity $(W / m \cdot K)$, Temperature

$\begin{array}{llll}5.0540 \mathrm{e}+001,1.0 & 4.2006 \mathrm{e}+002,101.0 & 3.8950 \mathrm{e}+002,201.0 & 3.8652 \mathrm{e}+002,301.0 \\ 4.9856 \mathrm{e}+002,11.0 & 4.1364 \mathrm{e}+002,111.0 & 3.8916 \mathrm{e}+002,211.0 & 3.8628 \mathrm{e}+002,311.0 \\ 8.6654 \mathrm{e}+002,21.0 & 4.0817 \mathrm{e}+002,121.0 & 3.8902 \mathrm{e}+002,221.0 & 3.8606 \mathrm{e}+002,321.0 \\ 9.9310 \mathrm{e}+002,31.0 & 4.0357 \mathrm{e}+002,131.0 & 3.8899 \mathrm{e}+002,231.0 & 3.8585 \mathrm{e}+002,331.0 \\ 8.8242 \mathrm{e}+002,41.0 & 3.9977 \mathrm{e}+002,141.0 & 3.8899 \mathrm{e}+002,241.0 & 3.8566 \mathrm{e}+002,341.0 \\ 7.1324 \mathrm{e}+002,51.0 & 3.9669 \mathrm{e}+002,151.0 & 3.8896 \mathrm{e}+002,251.0 & 3.8547 \mathrm{e}+002,351.0 \\ 5.8975 \mathrm{e}+002,61.0 & 3.9427 \mathrm{e}+002,161.0 & 3.8882 \mathrm{e}+002,261.0 & 3.8529 \mathrm{e}+002,361.0 \\ 5.0690 \mathrm{e}+002,71.0 & 3.9241 \mathrm{e}+002,171.0 & 3.8849 \mathrm{e}+002,271.0 & 3.8511 \mathrm{e}+002,371.0 \\ 4.6132 \mathrm{e}+002,81.0 & 3.9104 \mathrm{e}+002,181.0 & 3.8789 \mathrm{e}+002,281.0 & 3.8494 \mathrm{e}+002,381.0 \\ 4.3484 \mathrm{e}+002,91.0 & 3.9010 \mathrm{e}+002,191.0 & 3.8696 \mathrm{e}+002,291.0 & 3.8476 \mathrm{e}+002,391.0\end{array}$


Appendix B: The temperature-dependent material property for Aluminum and Copper

$\begin{array}{llll}3.8459 \mathrm{e}+002,401.0 & 3.7606 \mathrm{e}+002,651.0 & 3.5898 \mathrm{e}+002,901.0 & 3.4220 \mathrm{e}+002,1151.0 \\ 3.8440 \mathrm{e}+002,411.0 & 3.7550 \mathrm{e}+002,661.0 & 3.5824 \mathrm{e}+002,911.0 & 3.4159 \mathrm{e}+002,1161.0 \\ 3.8422 \mathrm{e}+002,421.0 & 3.7493 \mathrm{e}+002,671.0 & 3.5751 \mathrm{e}+002,921.0 & 3.4099 \mathrm{e}+002,1171.0 \\ 3.8402 \mathrm{e}+002,431.0 & 3.7434 \mathrm{e}+002,681.0 & 3.5677 \mathrm{e}+002,931.0 & 3.4038 \mathrm{e}+002,1181.0 \\ 3.8382 \mathrm{e}+002,441.0 & 3.7373 \mathrm{e}+002,691.0 & 3.5605 \mathrm{e}+002,941.0 & 3.3977 \mathrm{e}+002,1191.0 \\ 3.8360 \mathrm{e}+002,451.0 & 3.7312 \mathrm{e}+002,701.0 & 3.5533 \mathrm{e}+002,951.0 & 3.3916 \mathrm{e}+002,1201.0 \\ 3.8338 \mathrm{e}+002,461.0 & 3.7249 \mathrm{e}+002,711.0 & 3.5461 \mathrm{e}+002,961.0 & 3.3854 \mathrm{e}+002,1211.0 \\ 3.8314 \mathrm{e}+002,471.0 & 3.7184 \mathrm{e}+002,721.0 & 3.5390 \mathrm{e}+002,971.0 & 3.3792 \mathrm{e}+002,1221.0 \\ 3.8289 \mathrm{e}+002,481.0 & 3.7119 \mathrm{e}+002,731.0 & 3.5319 \mathrm{e}+002,981.0 & 3.3728 \mathrm{e}+002,1231.0 \\ 3.8262 \mathrm{e}+002,491.0 & 3.7052 \mathrm{e}+002,741.0 & 3.5249 \mathrm{e}+002,991.0 & 3.3664 \mathrm{e}+002,1241.0 \\ 3.8234 \mathrm{e}+002,501.0 & 3.6985 \mathrm{e}+002,751.0 & 3.5180 \mathrm{e}+002,1001.0 & 3.3598 \mathrm{e}+002,1251.0 \\ 3.8204 \mathrm{e}+002,511.0 & 3.6916 \mathrm{e}+002,761.0 & 3.5112 \mathrm{e}+002,1011.0 & 3.3530 \mathrm{e}+002,1261.0 \\ 3.8173 \mathrm{e}+002,521.0 & 3.6846 \mathrm{e}+002,771.0 & 3.5044 \mathrm{e}+002,1021.0 & 3.3461 \mathrm{e}+002,1271.0 \\ 3.8140 \mathrm{e}+002,531.0 & 3.6776 \mathrm{e}+002,781.0 & 3.4977 \mathrm{e}+002,1031.0 & 3.3389 \mathrm{e}+002,1281.0 \\ 3.8105 \mathrm{e}+002,541.0 & 3.6705 \mathrm{e}+002,791.0 & 3.4910 \mathrm{e}+002,1041.0 & 3.3316 \mathrm{e}+002,1291.0 \\ 3.8069 \mathrm{e}+002,551.0 & 3.6633 \mathrm{e}+002,801.0 & 3.4844 \mathrm{e}+002,1051.0 & 3.3239 \mathrm{e}+002,1301.0 \\ 3.8030 \mathrm{e}+002,561.0 & 3.6561 \mathrm{e}+002,811.0 & 3.4779 \mathrm{e}+002,1061.0 & 3.3160 \mathrm{e}+002,1311.0 \\ 3.7990 \mathrm{e}+002,571.0 & 3.6488 \mathrm{e}+002,821.0 & 3.4715 \mathrm{e}+002,1071.0 & 3.3078 \mathrm{e}+002,1321.0 \\ 3.7948 \mathrm{e}+002,581.0 & 3.6415 \mathrm{e}+002,831.0 & 3.4651 \mathrm{e}+002,1081.0 & 3.2992 \mathrm{e}+002,1331.0 \\ 3.7905 \mathrm{e}+002,591.0 & 3.6341 \mathrm{e}+002,841.0 & 3.4588 \mathrm{e}+002,1091.0 & 3.2902 \mathrm{e}+002,1341.0 \\ 3.7859 \mathrm{e}+002,601.0 & 3.6267 \mathrm{e}+002,851.0 & 3.4526 \mathrm{e}+002,1101.0 & 3.2807 \mathrm{e}+002,1351.0 \\ 3.7812 \mathrm{e}+002,611.0 & 3.6193 \mathrm{e}+002,861.0 & 3.4464 \mathrm{e}+002,1111.0 & 3.2739 \mathrm{e}+002,1358.0 \\ 3.7763 \mathrm{e}+002,621.0 & 3.6119 \mathrm{e}+002,871.0 & 3.4402 \mathrm{e}+002,1121.0 & \\ 3.7712 \mathrm{e}+002,631.0 & 3.6045 \mathrm{e}+002,881.0 & 3.4341 \mathrm{e}+002,1131.0 & \\ 3.7660 \mathrm{e}+002,641.0 & 3.5971 \mathrm{e}+002,891.0 & 3.4280 \mathrm{e}+002,1141.0 & \\ & & & \end{array}$

\section{Specific heat of Copper}

Specific heat $(J / k g \cdot K)$, Temperature

\begin{abstract}
$1.2961 \mathrm{e}-002,1.0$
$1.1439 \mathrm{e}+000,11.0$

$8.2366 \mathrm{e}+000,21.0$

$2.9369 \mathrm{e}+001,31.0$

$6.2167 \mathrm{e}+001,41.0$

$1.0060 \mathrm{e}+002,51.0$

$1.3848 \mathrm{e}+002,61.0$

$1.7372 \mathrm{e}+002,71.0$

$2.0417 \mathrm{e}+002,81.0$

$2.3035 \mathrm{e}+002,91.0$

$2.5277 \mathrm{e}+002,101.0$
\end{abstract}

$2.7189 \mathrm{e}+002,111.0$

$2.8813 \mathrm{e}+002,121.0$

$3.0190 \mathrm{e}+002,131.0$

$3.1355 \mathrm{e}+002,141.0$

$3.2342 \mathrm{e}+002,151.0$

$3.3181 \mathrm{e}+002,161.0$

$3.3898 \mathrm{e}+002,171.0$

$3.4517 \mathrm{e}+002,181.0$

$3.5058 \mathrm{e}+002,191.0$

$3.5537 \mathrm{e}+002,201.0$

$3.5970 \mathrm{e}+002,211.0$
$3.6365 \mathrm{e}+002,221.0$

$3.6730 \mathrm{e}+002,231.0$

$3.7069 \mathrm{e}+002,241.0$

$3.7382 \mathrm{e}+002,251.0$

$3.7667 \mathrm{e}+002,261.0$

$3.7918 \mathrm{e}+002,271.0$

$3.8126 \mathrm{e}+002,281.0$

$3.8278 \mathrm{e}+002,291.0$

$3.8362 \mathrm{e}+002,301.0$

$3.8488 \mathrm{e}+002,311.0$

$3.8612 \mathrm{e}+002,321.0$
$3.8735 \mathrm{e}+002,331.0$

$3.8856 \mathrm{e}+002,341.0$

$3.8977 \mathrm{e}+002,351.0$

$3.9096 \mathrm{e}+002,361.0$

$3.9213 \mathrm{e}+002,371.0$

$3.9329 \mathrm{e}+002,381.0$

$3.9444 \mathrm{e}+002,391.0$

$3.9557 \mathrm{e}+002,401.0$

$3.9669 \mathrm{e}+002,411.0$

$3.9779 \mathrm{e}+002,421.0$

$3.9888 \mathrm{e}+002,431.0$ 
Appendix B: The temperature-dependent material property for Aluminum and Copper

$\begin{array}{llll}3.9996 \mathrm{e}+002,441.0 & 4.2026 \mathrm{e}+002,661.0 & 4.3756 \mathrm{e}+002,881.0 & 4.6175 \mathrm{e}+002,1101.0 \\ 4.0102 \mathrm{e}+002,451.0 & 4.2106 \mathrm{e}+002,671.0 & 4.3842 \mathrm{e}+002,891.0 & 4.6326 \mathrm{e}+002,1111.0 \\ 4.0207 \mathrm{e}+002,461.0 & 4.2186 \mathrm{e}+002,681.0 & 4.3928 \mathrm{e}+002,901.0 & 4.6481 \mathrm{e}+002,1121.0 \\ 4.0310 \mathrm{e}+002,471.0 & 4.2264 \mathrm{e}+002,691.0 & 4.4016 \mathrm{e}+002,911.0 & 4.6641 \mathrm{e}+002,1131.0 \\ 4.0411 \mathrm{e}+002,481.0 & 4.2343 \mathrm{e}+002,701.0 & 4.4106 \mathrm{e}+002,921.0 & 4.6806 \mathrm{e}+002,1141.0 \\ 4.0512 \mathrm{e}+002,491.0 & 4.2420 \mathrm{e}+002,711.0 & 4.4197 \mathrm{e}+002,931.0 & 4.6977 \mathrm{e}+002,1151.0 \\ 4.0611 \mathrm{e}+002,501.0 & 4.2498 \mathrm{e}+002,721.0 & 4.4291 \mathrm{e}+002,941.0 & 4.7153 \mathrm{e}+002,1161.0 \\ 4.0708 \mathrm{e}+002,511.0 & 4.2575 \mathrm{e}+002,731.0 & 4.4387 \mathrm{e}+002,951.0 & 4.7336 \mathrm{e}+002,1171.0 \\ 4.0804 \mathrm{e}+002,521.0 & 4.2652 \mathrm{e}+002,741.0 & 4.4485 \mathrm{e}+002,961.0 & 4.7524 \mathrm{e}+002,1181.0 \\ 4.0899 \mathrm{e}+002,531.0 & 4.2729 \mathrm{e}+002,751.0 & 4.4585 \mathrm{e}+002,971.0 & 4.7719 \mathrm{e}+002,1191.0 \\ 4.0993 \mathrm{e}+002,541.0 & 4.2805 \mathrm{e}+002,761.0 & 4.4688 \mathrm{e}+002,981.0 & 4.7920 \mathrm{e}+002,1201.0 \\ 4.1085 \mathrm{e}+002,551.0 & 4.2882 \mathrm{e}+002,771.0 & 4.4793 \mathrm{e}+002,991.0 & 4.8127 \mathrm{e}+002,1211.0 \\ 4.1176 \mathrm{e}+002,561.0 & 4.2959 \mathrm{e}+002,781.0 & 4.4901 \mathrm{e}+002,1001.0 & 4.8342 \mathrm{e}+002,1221.0 \\ 4.1266 \mathrm{e}+002,571.0 & 4.3036 \mathrm{e}+002,791.0 & 4.5013 \mathrm{e}+002,1011.0 & 4.8563 \mathrm{e}+002,1231.0 \\ 4.1355 \mathrm{e}+002,581.0 & 4.3113 \mathrm{e}+002,801.0 & 4.5127 \mathrm{e}+002,1021.0 & 4.8792 \mathrm{e}+002,1241.0 \\ 4.1442 \mathrm{e}+002,591.0 & 4.3191 \mathrm{e}+002,811.0 & 4.5245 \mathrm{e}+002,1031.0 & 4.9028 \mathrm{e}+002,1251.0 \\ 4.1528 \mathrm{e}+002,601.0 & 4.3270 \mathrm{e}+002,821.0 & 4.5366 \mathrm{e}+002,1041.0 & 4.9273 \mathrm{e}+002,1261.0 \\ 4.1614 \mathrm{e}+002,611.0 & 4.3348 \mathrm{e}+002,831.0 & 4.5491 \mathrm{e}+002,1051.0 & 4.9525 \mathrm{e}+002,1271.0 \\ 4.1698 \mathrm{e}+002,621.0 & 4.3428 \mathrm{e}+002,841.0 & 4.5619 \mathrm{e}+002,1061.0 & 4.9785 \mathrm{e}+002,1281.0 \\ 4.1781 \mathrm{e}+002,631.0 & 4.3509 \mathrm{e}+002,851.0 & 4.5752 \mathrm{e}+002,1071.0 & 5.0053 \mathrm{e}+002,1291.0 \\ 4.1864 \mathrm{e}+002,641.0 & 4.3590 \mathrm{e}+002,861.0 & 4.5889 \mathrm{e}+002,1081.0 & 5.0303 \mathrm{e}+002,1300.0 \\ 4.1945 \mathrm{e}+002,651.0 & 4.3673 \mathrm{e}+002,871.0 & 4.6030 \mathrm{e}+002,1091.0 & \end{array}$




\section{Appendix C: Comparison of Analysis Procedures}

In this study, the Dynamic-Explicit procedure including the adiabatic heating effects (step1), and the Dynamic Temperature-Displacement Explicit procedure (step2) available in ABAQUS are used to clarify the influence of analysis procedure.

For easy to compare the results, all the set-up information is same for two different procedures; i.e., the diameter is 25 , the velocity is $500 \mathrm{~m} / \mathrm{s}$, the friction coefficient is 0.3 , the initial temperature of particle and substrate are both $300 \mathrm{~K}$. The total solution time is set as $60 \mathrm{~ns}$.

\section{Results and discussion}

The contour of equivalent plastic strain (PEEQ) and temperature (TEMP) obtained by using the two analysis procedures are show in FIG.AC.1 and FIG.AC.2, respectively. The evolution of the equivalent plastic strain and temperature for the key element is shown in FIG.AC.3. The maximum value of PEEQ is 22.1 for step 1, 4.8 for step 2; and the maximum value of temperature is $2327.4 \mathrm{~K}$ for step $1,477.5 \mathrm{~K}$ for step 2.

Firstly, the simulation results of PEEQ and TEMP obtained by using the DynamicExplicit procedure including the adiabatic heating are significantly higher than that obtained by using the second procedure (step2), which beyond the reasonable range. The experimental result shows that the critical velocity of Al is $766 \mathrm{~m} / \mathrm{s}$, which means that the adiabatic shear instability appears at and above this impact velocity. The impact velocity of $500 \mathrm{~m} / \mathrm{s}$ is applied in the study of this section, thus there is no way to observe the second rise of PEEQ and the extremely high temperature, even higher than its melting temperature. Secondly, the deformation shape predicted by using the two different analysis procedures are different. The outer region of the interface between particle and substrate deforms into a small and thin jet-region by using the first analysis procedure, however, in FIG.AC.2, this phenomenon does not exist. Such excessive mesh distortion leads to convergence difficulty, and should be avoid as much as possible in the calculations.

Based on the above discussion, the Dynamic Temp-Disp Explicit procedure is chosen for the next investigation of effects of various parameters. 
(a)

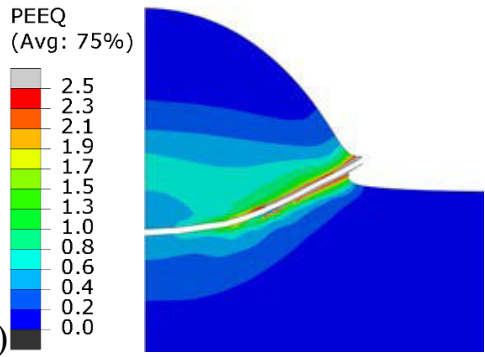

(b)

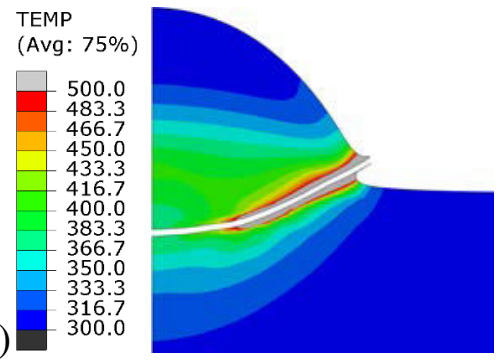

Figure AC.1: Simulated contours of PEEQ (a) and TEMP (b) of Al/Al using the Dynamic-Explicit including adiabatic heating procedure.
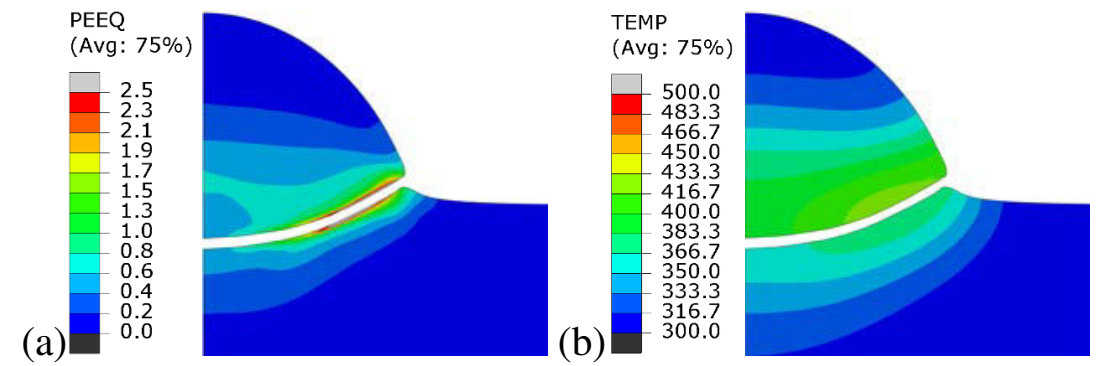

Figure AC.2: Simulated contours of PEEQ (a) and TEMP (b) of Al/Al using the Dynamic Temp-Disp Explicit procedure.

(a)

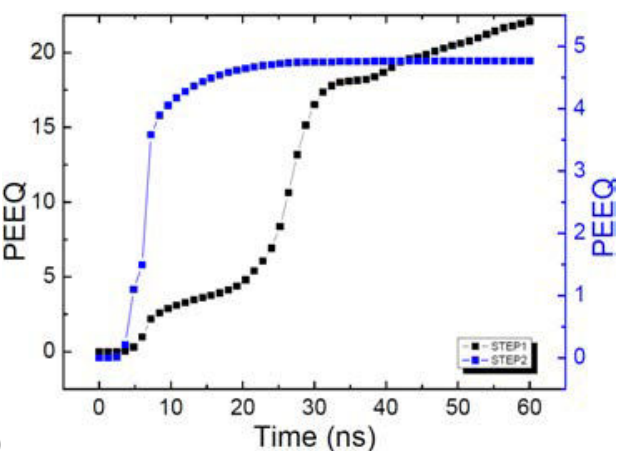

(b)

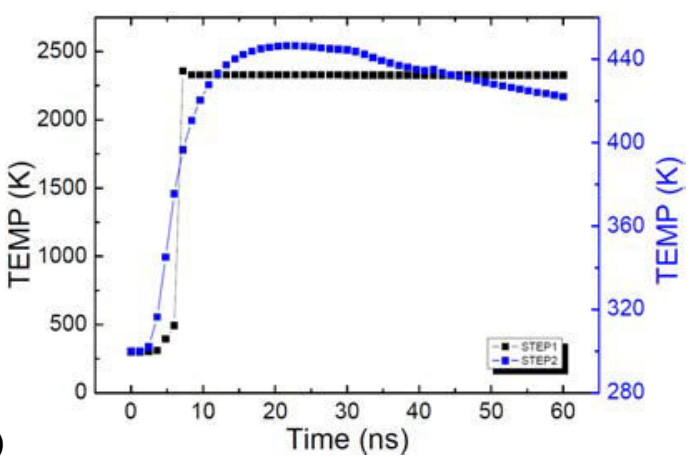

(b) TEMP of Al/Al by using two different analysis procedures at $500 \mathrm{~m} / \mathrm{s}$. 


\section{Appendix D: Contact pair algorithm in ABAQUS/Explicit}

\section{Contact properties}

\section{Mechanical contact properties}

The contact pressure-overclosure relationship used in this study is referred to as the 'hard' contact model. Hard contact implies that:

- The surfaces transmit no contact pressure unless the nodes of the slave surface contact the master surface;

- No penetration is allowed at each constraint location (depending on the constraint enforcement method used, this condition will either be strictly satisfied or approximated);

- There is no limit to the magnitude of contact pressure that can be transmitted when the surfaces are in contact.

The classical isotropic Coulomb friction model is used in this study for modeling the friction relationship between particle and substrate. The basic concept of the Coulomb friction model is to relate the maximum allowable frictional (shear) stress across an interface to the contact pressure between the contacting bodies. In the basic form of the Coulomb friction model, two contacting surfaces can carry shear stresses up to a certain magnitude across their interface before they start sliding relative to one another; this state is known as sticking. The Coulomb friction model defines this critical shear stress, $\tau_{\text {crit }}$, at which sliding of the surfaces starts as a fraction of the contact pressure, $p$, between the surfaces $\left(\tau_{c r i t}=\mu p\right)$. The stick/slip calculations determine when a point transitions from sticking to slipping or from slipping to sticking. The fraction, $\mu$, is known as the coefficient of friction as FIG.AD.1 shows. The basic friction model assumes that $\mu$ is the same in all directions (isotropic friction).

\section{Thermal contact properties}

In the cold spray particle contact case, the heating is assumed to be adiabatic, i.e., heat transfer is not considered. In modelling of high strain rate phenomena, the validity of this assumption can be assessed with respect to the value of the dimensionless parameter 


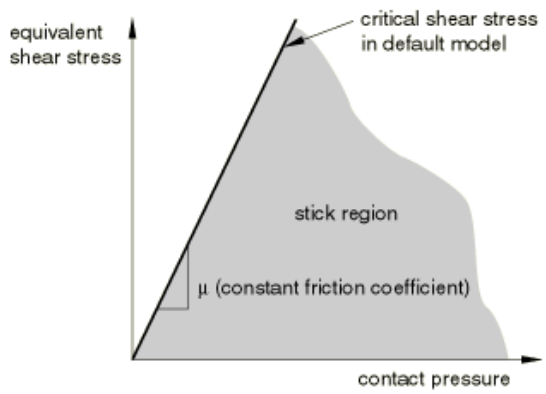

Figure AD.1: Schematic diagram of basic Coulomb friction model.

$x^{2} / D_{t h} t$, in which $x$ is a characteristic system dimension, $D_{t h}$ is thermal diffusivity and $t$ is the process time [ASS 03]. A value of $x^{2} / D_{t h} t$ equal or above unity is considered to justify the assumption of adiabatic heating. The value of this parameter can be shown to be above unity for the case of particle impact in cold gas spraying, by taking typical values of $10^{-6} \mathrm{~m}^{2} / \mathrm{s}$ for $D_{t h}, 40 \mathrm{~ns}$ for $t$ and $1 \mu \mathrm{m}$ for the element size in a particle of $25 \mu \mathrm{m}$ in diameter. At very small length scales, heat conduction would be dominated by a wave propagation mechanism rather than by diffusion. This implies that with decreasing particle size, the speed of heat propagation would approach that of plastic waves, and would in any case be limited by the speed of sound in the particle. In other words, for high speed impacts of much smaller particles, heat conduction could be even slower than what is predicted by the diffusive heat equation. Therefore, it could still be reasonable to assume adiabatic heating even for the impact of very small particles. It has also been suggested that the dissipation of kinetic energy into heat is strain rate dependent, i.e., the fraction of plastic work dissipated into heat would be larger for higher strain rates. This would further support the assumption of adiabatic heating in cold gas sprayed particles. Therefore, the thermal contact properties of conductance and radiation are ignored in this study.

\section{Contact formulations}

The contact formulation for the contact pair algorithm in ABAQUS/Explicit includes:

- The contact surface weighting (balanced or pure master-slave);

- The sliding formulation (finite, small, or infinitesimal).

\section{Contact surface weighting}

Both the pure master-slave and the balanced master-slave contact algorithms are available in ABAQUS/Explicit. Generally, contact constraints in a finite element model are applied in a discrete manner, meaning that for hard contact a node on one surface is constrained to not penetrate the other surface. In pure master-slave contact, the node with the constraint is part of the slave surface, and the nodes on the master surface can, in prin- 
ciple, penetrate the slave surface unhindered (see FIG.AD.2). For balanced master-slave contact ABAQUS/Explicit calculates the contact constraints twice for each set of surfaces in contact, in the form of penalty forces: once with the first surface acting as the master surface and once with the second surface acting as the master surface. The weighted average of the two corrections (or forces) is applied to the contact interaction. Balanced master-slave contact minimizes the penetration of the contacting bodies and, thus, provides better enforcement of contact constraints and more accurate results in most cases. For two element-based deformable surfaces contact each other, the balanced masterslave weighting is used.

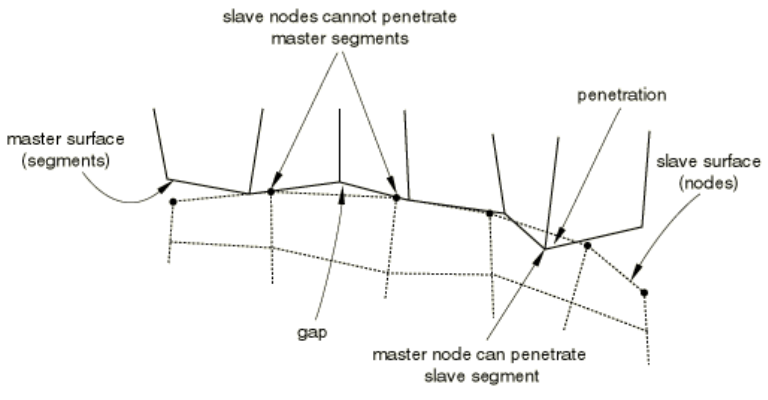

Figure AD.2: Schematic diagram of master surface penetrations into the slave surface.

\section{Sliding formulation}

In ABAQUS/Explicit there are three approaches to account for the relative motion of the two surfaces forming a contact pair:

- Finite sliding, which is the most general and allows any arbitrary motion of the surfaces;

- Small sliding, which assumes that although two bodies may undergo large motions, there will be relatively little sliding of one surface along the other;

- Infinitesimal sliding and rotation, which assumes that both the relative motion of the surfaces and the absolute motion of the contacting bodies are small.

Therefore, due to the nonlinear geometric effect of particle, one chooses the Finite sliding formulation in this study. In FIG.AD.3 node 101 of slave surface ASURF may come into contact anywhere along the master surface $B S U R F$. While in contact, it is constrained to slide along $B S U R F$, irrespective of the orientation and deformation of this surface. Node 101 is in contact with the element face with end nodes 201 and 202 at time $t_{1}$. The load transfer at this time occurs between node 101 and nodes 201 and 202 only. Later on, at time $t_{2}$, node 101 may find itself in contact with the element face with end nodes 501 and 502. Then the load transfer will occur between node 101 and nodes 501 and 502. This is a possible evolution of the contact between node 101 and its master surface $B S U R F$. 


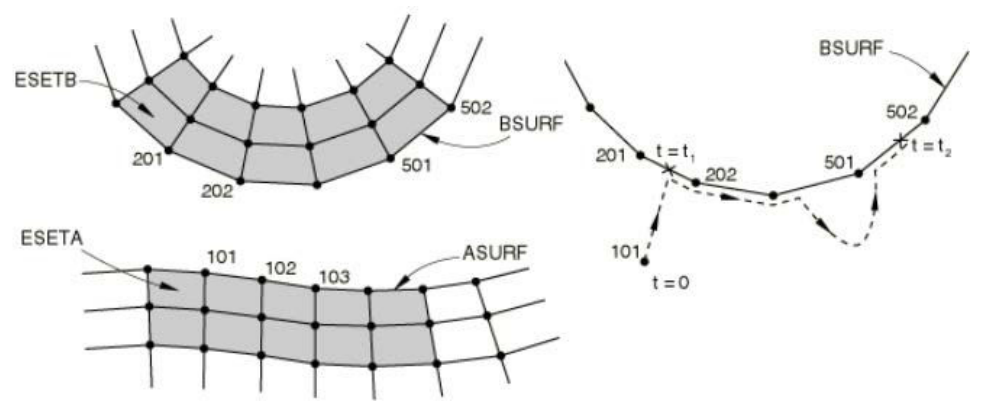

Figure AD.3: Schematic diagram of finite sliding formulation.

\section{Contact tracking algorithms}

Finite-sliding tracking is designed to be robust, yet computationally efficient. This algorithm assumes that the incremental relative tangential motion between surfaces does not significantly exceed the dimensions of the master surface facets, but there is no limit to the overall relative motion between surfaces. The contact search algorithm uses a global search at the beginning of each step, and a hierarchical global/local search algorithm is used for the other increments. For a balanced master-slave contact pair, the contact search computations are performed twice for each contact pair.

The global search, as FIG.AD.4(a) illustrated, computes the distance from node 50 to all of the master surface facets in the same bucket as node 50. It determines that the nearest facet on the master surface to node 50 is the facet of element 10 . Node 100 is the node on this facet that is nearest to node 50, and it is designated the tracked master surface node. This search is conducted for each slave node, comparing each node against all of the facets on the master surface that are in the same bucket. By default, ABAQUS/Explicit performs a global search every one hundred increments for two-surface contact pairs.

In the local search shown in FIG.AD.4(b), node 50 moves as shown during an increment. In the first iteration of the search ABAQUS/Explicit finds that the master surface facet on element 10 is still the closest facet of those attached to node 100 but that node 101 is now the tracked master surface node. Because the previously tracked node was node 100, ABAQUS/Explicit performs another iteration. In this second iteration a new element, element 11 , is found to be the closest facet and the closest master surface node is 102. Another iteration is performed because the identity of the tracked master surface node changed. In the third iteration the identity of the tracked node does not change, so ABAQUS/Explicit designates node 102 as the tracked master surface node for slave node 50. A local search is substantially less expensive computationally than a global search.

\section{Contact constraint enforcement methods}

ABAQUS/Explicit uses two different methods to enforce contact constraints:

- The kinematic contact algorithm uses a kinematic predictor/corrector contact algorithm to strictly enforce contact constraints (for example, no penetrations are 

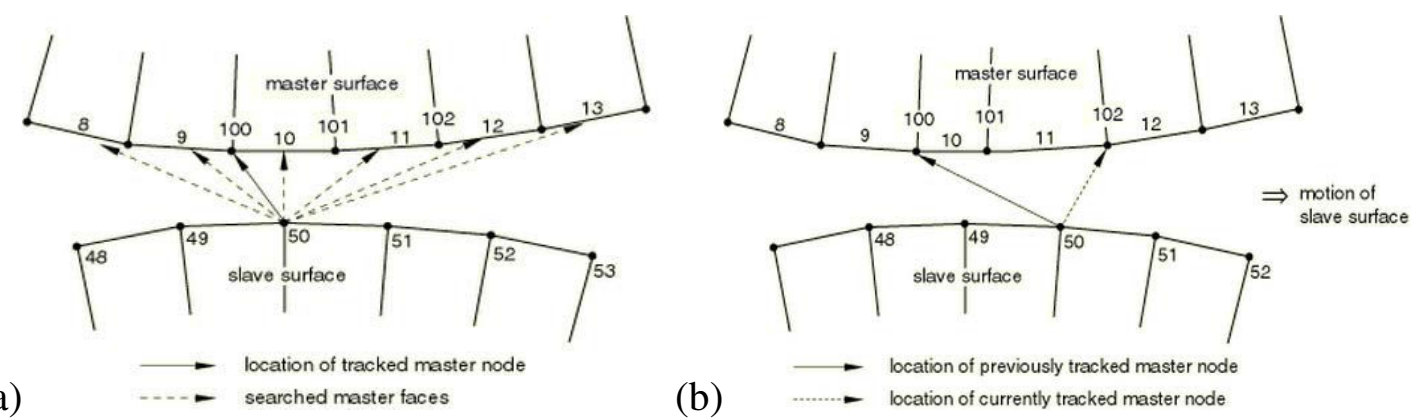

Figure AD.4: Schematic diagrams of (a) global search and (b) local search.

allowed);

- The penalty contact algorithm has a weaker enforcement of contact constraints but allows for treatment of more general types of contact.

For node-to-face contact, forces that are a function of the penetration distance are applied to the slave nodes to oppose the penetration, while equal and opposite forces act on the master surface at the penetration point. The master surface contact forces are distributed to the nodes of the master faces being penetrated. One set of forces is calculated considering one surface as the master surface, and the other forces are calculated considering that same surface as the slave surface. The penalty contact algorithm for balanced master-slave contact surfaces is to weight each of the two sets of forces equally. 


\section{Appendix E: ALE adaptive mesh controls}

\section{Mesh smoothing}

Mesh smoothing algorithms based only on minimizing element distortion tend to reduce the mesh refinement in areas of concave curvature, especially as the curvature evolves. Having sufficient mesh refinement near highly curved boundaries is often important to model both the shape and volume of the domain. To prevent the natural reduction in mesh refinement of areas near evolving concave curvature, ABAQUS/Explicit uses solution-dependent meshing to focus mesh gradation toward these areas automatically. The aggressiveness of the solution-dependent meshing is governed by the curvature refinement weight, $\alpha_{c}$. By default, $\alpha_{c}=1$, which corresponds to an aggressively level that has been chosen to work well on a wide variety of problems.

Volume smoothing relocates a node by computing a volume-weighted average of the element centers in the elements surrounding the node. In FIG.AE.1 the new position of node $M$ is determined by a volume-weighted average of the positions of the element centers, $C$, of the four surrounding elements. The volume weighting will tend to push the node away from element center $C_{1}$ and toward element center $C_{3}$, thus reducing element distortion. Volume smoothing is very robust and it works well for both structured and highly unstructured domains.

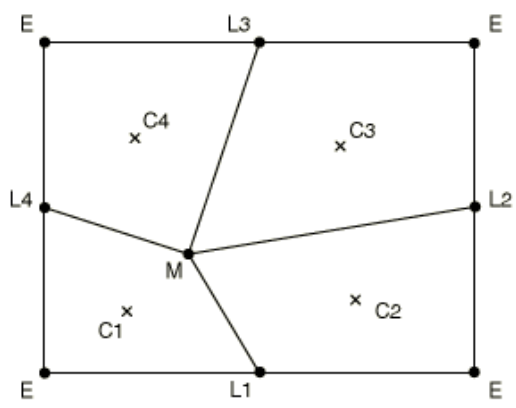

Figure AE.1: Schematic diagram of relocation of a node during a mesh sweep. 


\section{Boundary smoothing}

Adaptive mesh constraints must not conflict with other mesh constraints inherent to Lagrangian and sliding boundary regions; therefore, adaptive mesh constraints can be applied only tangentially to a Lagrangian or sliding boundary region. This restriction implies that adaptive mesh constraints can be applied only in two directions in a threedimensional boundary region, in one direction in a two-dimensional boundary region, or in one direction on a Lagrangian or active geometric edge. It may not always be feasible to adhere to this rule, particularly if the boundary experiences finite rotation. Therefore, if the normal to a boundary region is not perpendicular to a prescribed mesh constraint at a node, it is always moved along the current surface of the boundary region so that the projection of the mesh motion in the direction of the prescribed constraint is correct (see FIG.AE.2).

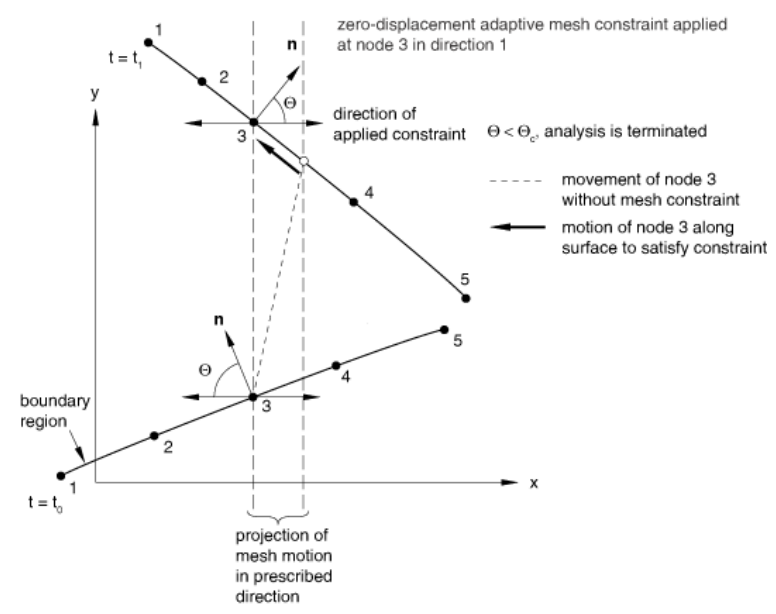

Figure AE.2: Schematic diagram of enforcing a spatial mesh constraint.

If the normal to the boundary region approaches the direction of the applied mesh constraint, large mesh motions will be required to satisfy the constraint. By default, an analysis is terminated if the angle between the normal to the boundary region and the direction of the prescribed constraint becomes less than the mesh constraint angle, $\theta_{C}=$ $60^{\circ}\left(5^{\circ} \leq \theta_{C} \leq 85^{\circ}\right)$. Since independent mesh motion cannot be prescribed perpendicular to these edges, an analysis is terminated if the angle between the prescribed constraint and the plane perpendicular to the edge falls below the specified mesh constraint angle.

\section{Advection}

An element variable, $\phi$, is remapped from the old mesh to the new mesh by first determining a linear distribution of the variable in each old element, as illustrated in FIG.AE.3 for a simple one-dimensional mesh. The linear distribution of $\phi$ in the middle element depends on the values of $\phi$ in the two adjacent elements. To construct the linear 
distribution:

1. A quadratic interpolation is constructed from the constant values of at the integration points of the middle element and in its adjacent elements.

2. A trial linear distribution, $\phi_{\text {trial }}$, is found by differentiating the quadratic function to find the slope at the integration point of the middle element.

3. The trial linear distribution in the middle element is limited by reducing its slope until its minimum and maximum values are within the range of the original constant values in the adjacent elements.

This process is referred to as flux-limiting and is essential to ensure that the advection is monotonic.

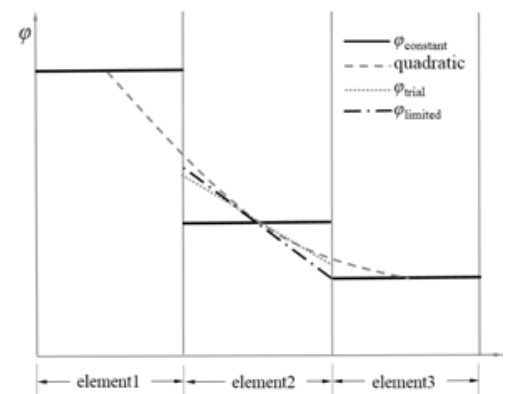

Figure AE.3: Schematic diagram of second-order advection. 
Appendix E: ALE adaptive mesh controls

150 


\section{Appendix F: Finite element conversion to SPH particles}

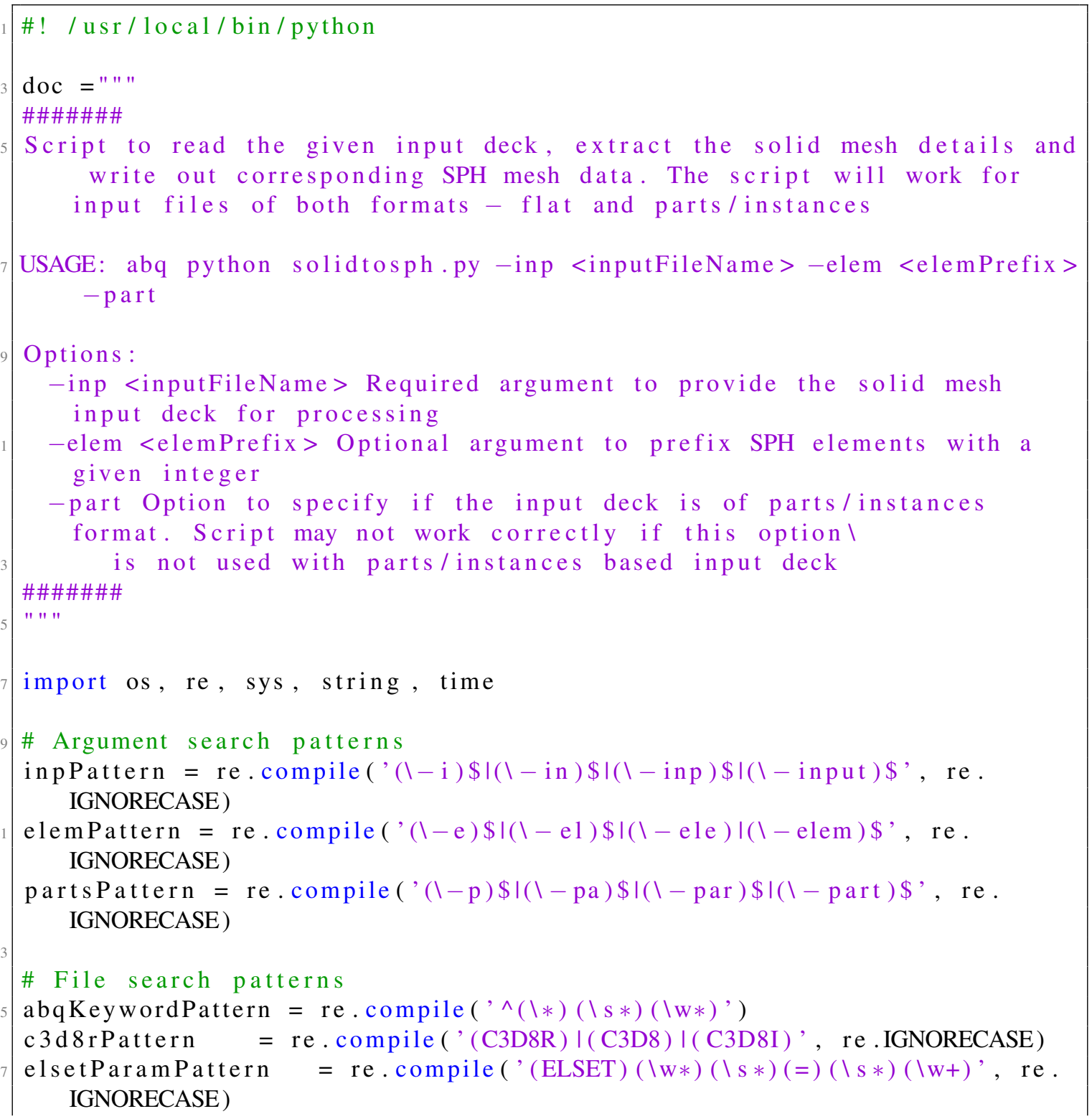




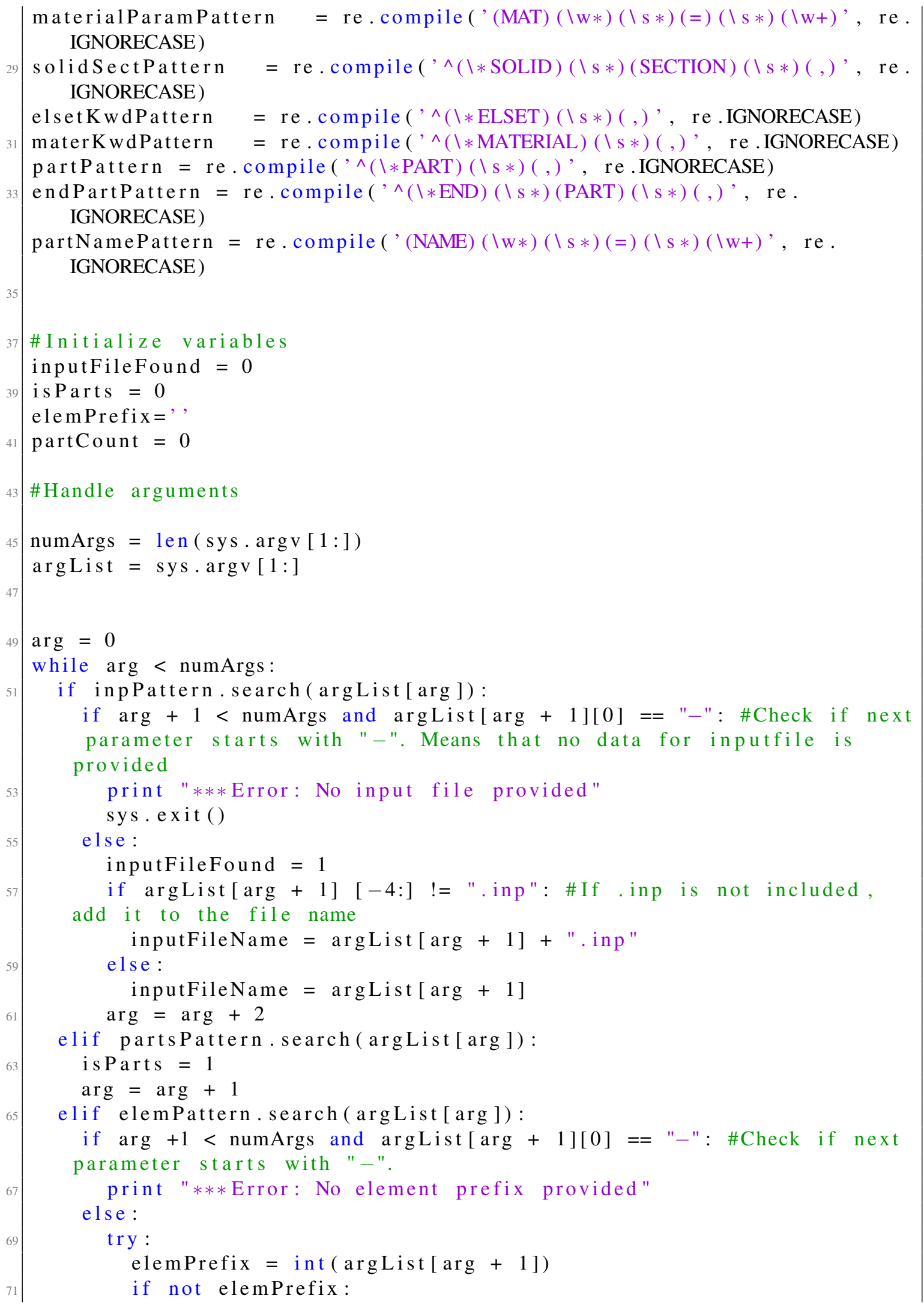




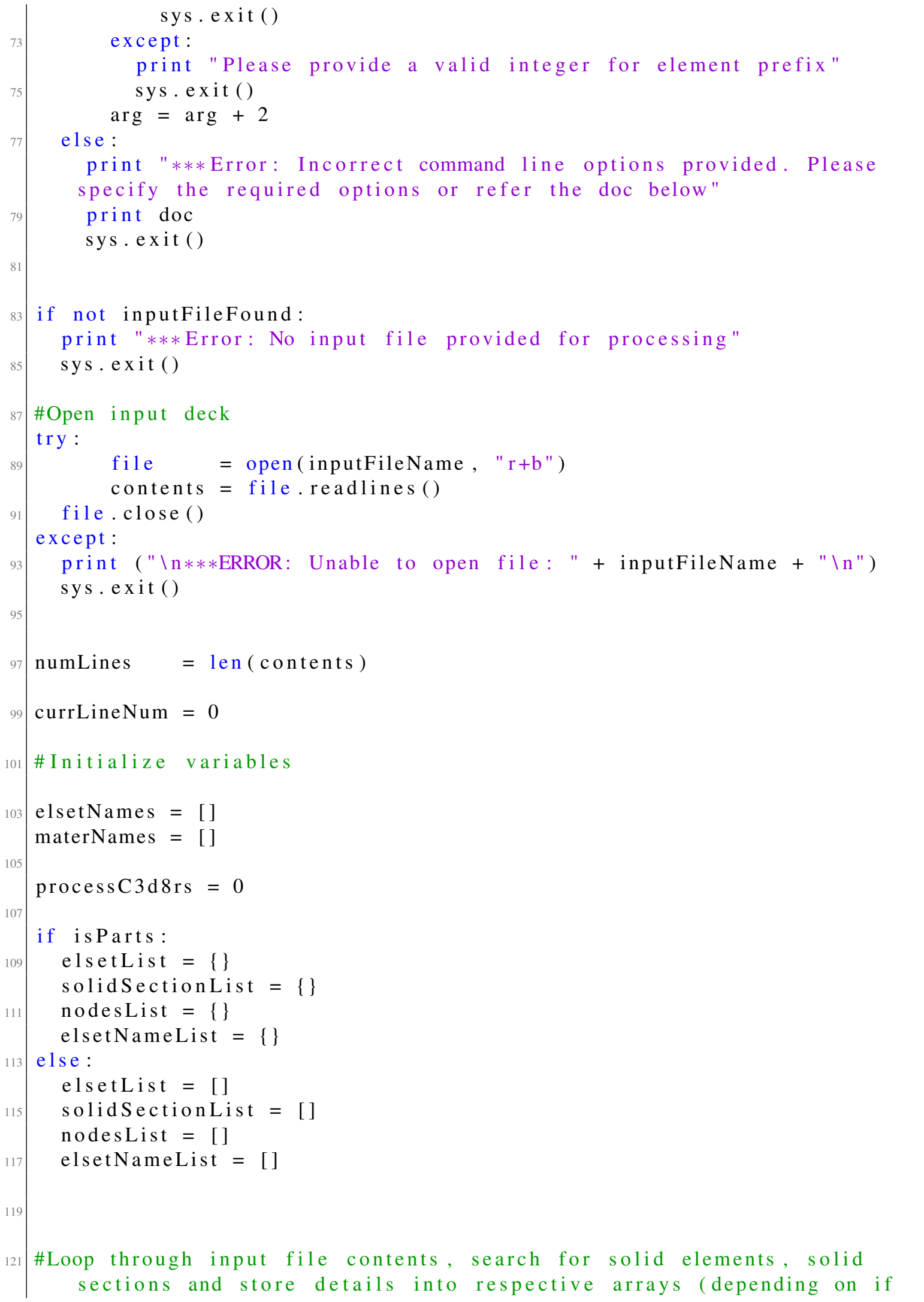




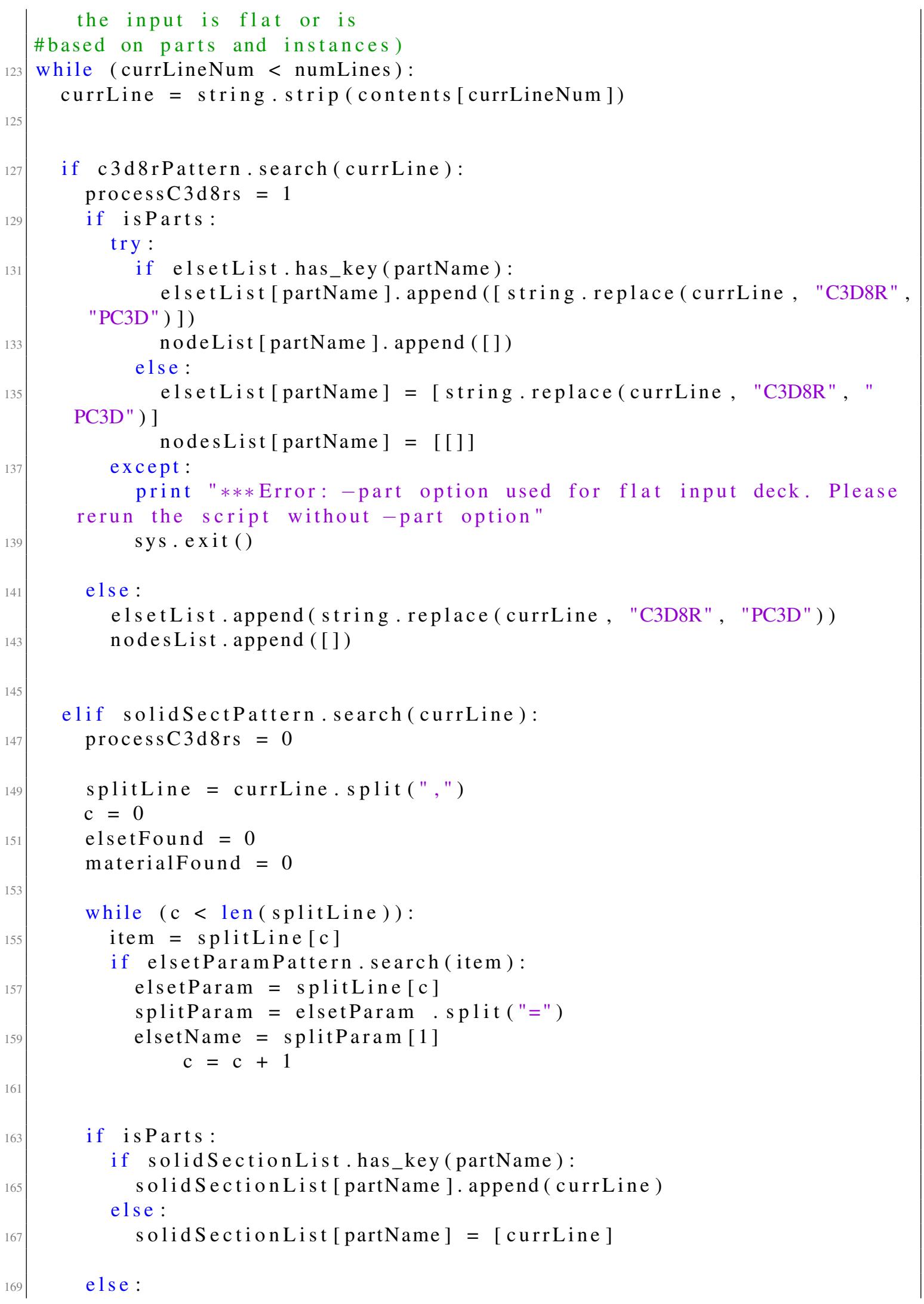




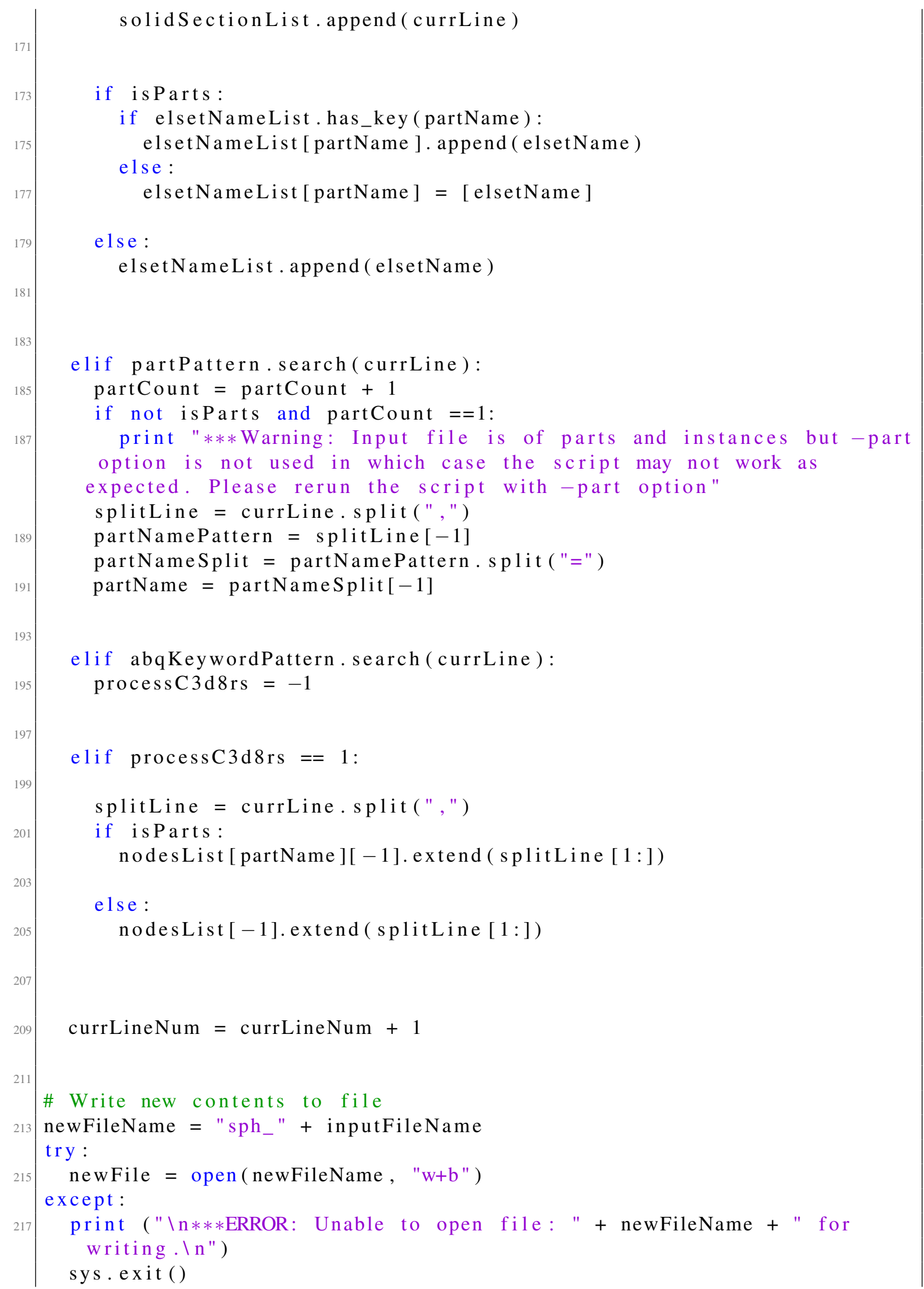


newLine $=$ ',

newFile. write (newLine $+" \backslash n "$ )

newLine $={ }^{*} *{ }_{*}-$ START SPH MESH $_{-}$

newFile. write (newLine $+" \backslash n "$ )

newLine $={ }^{*} * *$

newFile.write (newLine $+" \backslash n ")$

if is Parts :

for pNum in elsetList.keys():

newLine $=$ '*Part, Name $={ }^{\prime}+\operatorname{str}(\mathrm{pNum})$

newFile. write (newLine $+" \backslash n ")$

for eNum in range(len(elsetList[pNum])):

newLine $=$ elsetList $[\mathrm{pNum}][\mathrm{eNum}]$

newFile. write (newLine $+" \backslash n "$ )

nodesUniqSorted $=\operatorname{sorted}(\operatorname{set}($ nodesList $[\mathrm{pNum}][\mathrm{eNum}]))$

for nodeNum in nodesUniqSorted:

newLine $=\operatorname{str}($ elemPrefix $)+\operatorname{string} \cdot \operatorname{strip}($ nodeNum $)+{ }^{\prime},{ }_{+},{ }^{+}+$ str (nodeNum)

newFile. write (newLine $+" \backslash n ")$

newLine $={ }^{\prime} *$

newFile. write (newLine $+" \backslash n ")$

for solidSec in solidSectionList [pNum]:

newLine $=$ solidSec

newFile. write (newLine $+" \backslash n "$ )

newLine $=$ '< particleRadius $>$,'

newFile. write (newLine $+" \backslash n "$ )

newLine $=$

newFile. write (newLine $+" \backslash n ")$

for elsetName in elsetNameList[pNum]:

nSetName $=$ elsetName + ' ${ }^{-}$Nodes'

surfName $=$ elsetName + ' ${ }_{-}$Surf,

newLine $={ }^{\prime} *$ Nset, Nset $={ }^{\prime}+$ nSetName,+ , Elset $={ }^{\prime}+$ elsetName newFile. write (newLine $+" \backslash n "$ )

newLine $=$ ' $*$ Surface, Type $=$ Node, Name $=$ ' + surfName

newFile. write (newLine $+" \backslash n "$ )

newLine $=$ nSetName + , ,

newFile.write (newLine $+" \backslash n ")$

newLine $=$ ',

newFile. write (newLine $+" \backslash n "$ )

newLine $={ }^{\prime} *$ End Part ${ }^{\prime}$

newFile. write (newLine $+" \backslash n ")$

newLine $=$ '

newFile. write (newLine $+" \backslash n ")$ 


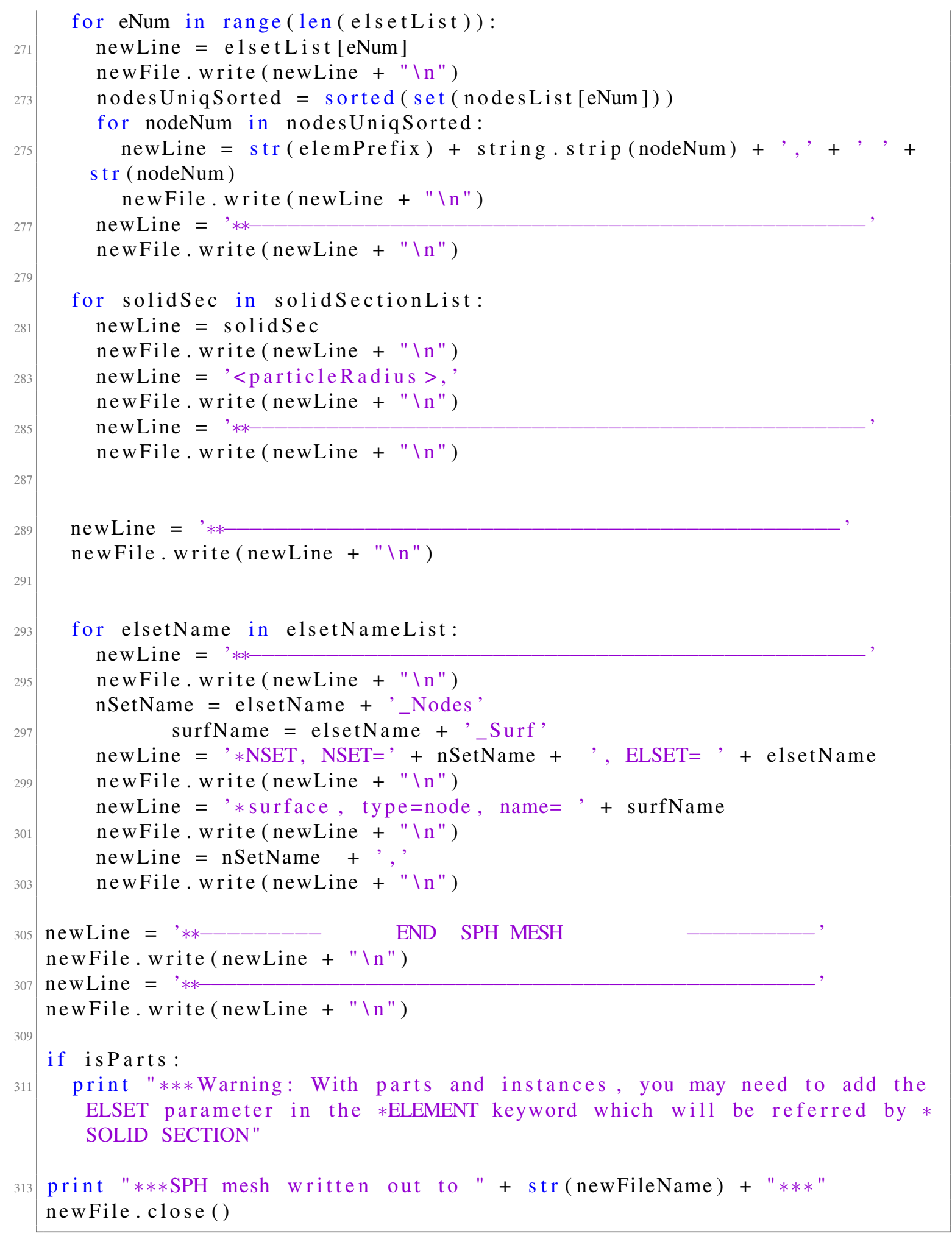

solidtosph.py 


\section{Appendix G: Assigning materials to Eulerian part}

\section{Volume fraction tool}

The procedure as following descriptions for how to use the volume fraction tool to assign materials to Eulerian part instances is shown in FIG.AG.1.

1. Using Part module in ABAQUS/CAE create a reference part that corresponds to the geometry of the desired Eulerian material region.

2. Instance the reference part within the Eulerian part instance. The reference part instance should spatially correspond to the desired Eulerian material region.

3. Use the volume fraction tool to create a discrete field based on a comparison of the reference part instance and the Eulerian part instance.

4. Define a material assignment predefined field for the Eulerian part instance using the discrete field created by the volume fraction tool.

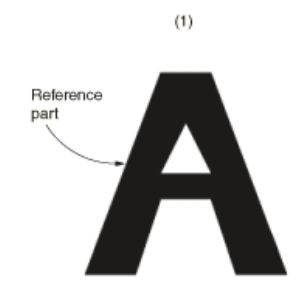

(3)

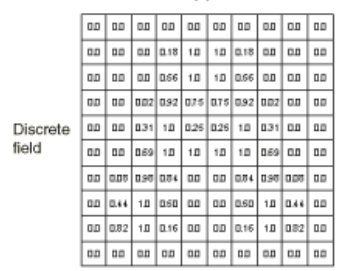

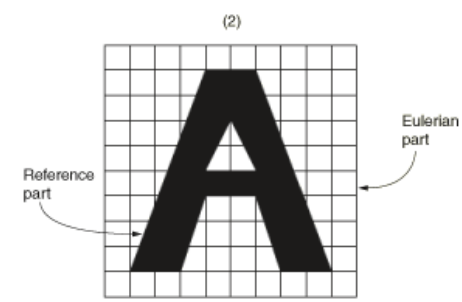

(4)

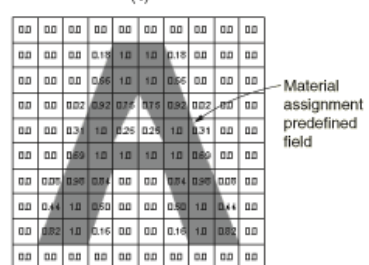

Figure AG.1: Procedure for using the volume fraction tool. 


\section{Appendix H: Modeling surface erosion}

The general contact algorithm modifies the list of contact faces and contact edges that are active in the contact domain based on the failure status of the underlying elements. General contact considers a face only if its underlying element has not failed and it is not coincident with a face from an adjacent element that has not failed; thus, exterior faces are initially active, and interior faces are initially inactive. Once an element fails, its faces are removed from the contact domain, and any interior faces that have been exposed are activated. A contact edge is removed when all the elements that contain the edge have failed. New contact edges are not created as elements erode. Based on this algorithm, the active contact domain evolves during the analysis as elements fail (see FIG.AH.1 for an example of an eroding solid).

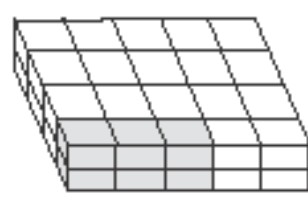

surtace topology before the shaded elements have fajled

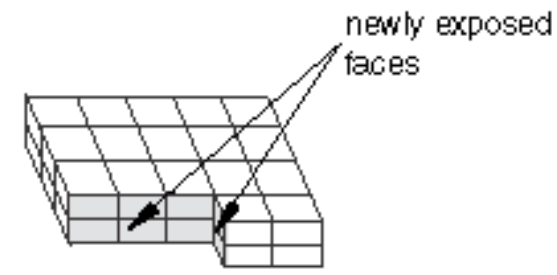

surface topology after failure

Figure AH.1: Topology of an eroding contact surface.

You can control whether contact nodes remain in the contact domain after all the surrounding elements have failed. By default, these nodes remain in the contact domain and act as free-floating point masses that can experience contact with faces that are still part of the contact domain. You can specify that nodes of element-based surfaces should erode once all contact faces and contact edges to which they are attached have eroded.

For a solid element mesh consisting of elements that may fail, every face that can potentially be involved in contact (both exterior and interior faces) should be included in the contact domain. The general contact algorithm will activate and deactivate faces as necessary when elements fail.

For example, you define an element set ELERODE that contains all the solid elements in the model that refer to a material failure model. First, you must create a surface SURFERODE containing all of the interior and exterior faces of these elements. You 
could define this surface using the automatic free surface and interior surface generation methods called Select from all entities available in the tool bar of ABAQUS/CAE.

Next, you must construct the contact domain. Defining 'automatic' general contact for the entire model is not sufficient because the contact domain created when this method is used does not include any interior faces. Therefore, you must define the pairwise interactions with the erodable surface explicitly in the contact inclusions definition. In the Edit included pairs window, you select (ALL*) as First surface, and (SURFERODE) as the second face.

The amount of contact data used to describe the surface topology is proportional to the number of faces included in the contact domain. Including a large number of interior faces in the contact domain can potentially increase memory use significantly compared to analyses in which the contact domain is defined using only exterior faces. Consider creating a surface on a cubic mesh of C3D8R elements with $n$ elements per side. A surface including the exterior faces of the mesh (suitable for modeling contact without element failure) would contain $6 n^{2}$ element faces. A surface including both exterior and interior faces of the mesh (suitable for modeling contact with element failure for every element in the mesh) would contain $6 n^{3}$ element faces. For large meshes the memory use can increase easily by an order of magnitude when interior element faces are included in the contact domain to model erosion. Therefore, it is recommended to include only those interior element faces in the contact domain that could possibly participate in contact. 


\section{Appendix I: Progressive damage and failure}

\section{General framework for modeling damage and failure}

ABAQUS/Explicit offers a general framework for material failure modeling. FIG.AI.1 illustrates a typical uni-axial stress-strain response of a ductile metal material. The material response is initially linear elastic, $a-b$, followed by plastic yielding with strain hardening, $b-c$. Beyond point $c$ there is a marked reduction of load-carrying capacity until rupture, $c-d$. In the FIG.AI. $1 \sigma_{y 0}$ and $\bar{\varepsilon}_{0}^{p l}$ are the yield stress and equivalent plastic strain at the onset of damage, and $\bar{\varepsilon}_{f}^{p l}$ is the equivalent plastic strain at failure; that is, when the overall damage variable reaches the value $D=1$.

When material damage occurs, the stress-strain relationship no longer accurately represents the material's behavior. Continuing to use the stress-strain relationship introduces a strong mesh dependency based on strain localization, such that the energy dissipated decreases as the mesh is refined. HILLERBORG [HIL 76] proposed to use the stressdisplacement relationship after damage instead of the stress - strain response curve. The implementation of this stress - displacement concept in a finite element model requires the definition of a characteristic length, $L$, associated with an integration point. The fracture energy required to open a unit area of crack is given as:

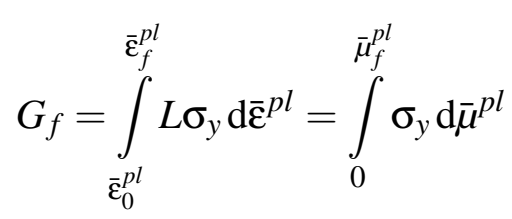

This expression introduces the definition of the equivalent plastic displacement, $\bar{\mu}^{p l}$, as the fracture work conjugate of the yield stress after the onset of damage (work per unit area of the crack). Before damage initiation $\dot{\bar{\mu}}^{p l}=0$; after damage initiation $\dot{\bar{\mu}}^{p l}=L \dot{\bar{\varepsilon}}^{p l}$.

Thus, in ABAQUS the specification of a failure mechanism consists of four distinct parts:

- the definition of the effective (or undamaged) material response $\left(a-b-c-d^{\prime}\right.$ in FIG.AI.1),

- a damage initiation criterion ( $c$ in FIG.AI.1),

- a damage evolution law ( $c-d$ in FIG.AI.1), 
- a choice of element deletion ( $d$ in FIG.AI.1).

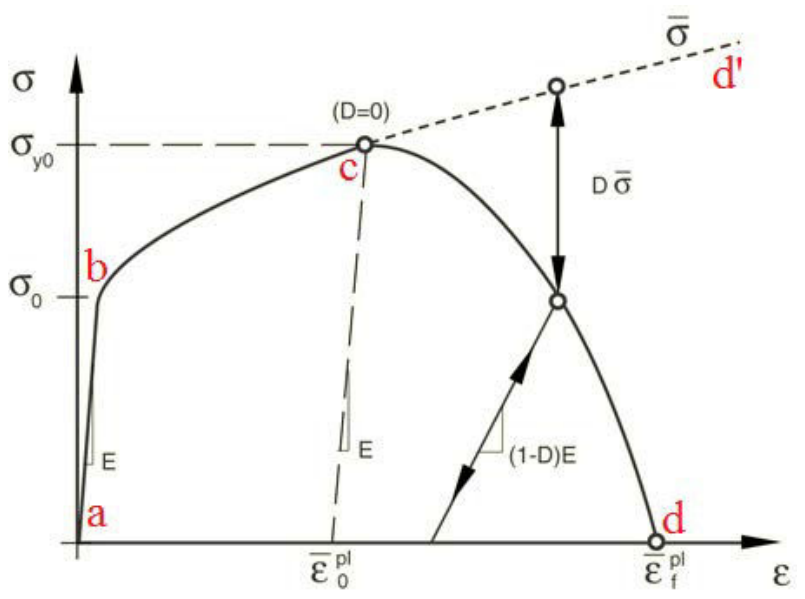

Figure AI.1: A typical uniaxial stress-strain response of a ductile metal specimen.

\section{Damage initiation criterion}

Two main mechanisms can cause the fracture of a ductile metal: ductile fracture due to the nucleation, growth, and coalescence of voids; and shear fracture due to shear band localization. The data of damage initiation criterion for Aluminum used in this section are displayed in Appendix J.

\section{Ductile criterion}

The ductile criterion is a phenomenological model for predicting the onset of damage due to nucleation, growth, and coalescence of voids. The model assumes that the equivalent plastic strain at the onset of damage, $\bar{\varepsilon}_{D}^{p l}$, is a function of stress triaxiality $\eta=-p / q$ and strain rate $\dot{\bar{\varepsilon}}^{p l}$.

The criterion for damage initiation is met when the following condition is satisfied:

$$
\omega_{D}=\int \frac{d \bar{\varepsilon}^{p l}}{\bar{\varepsilon}_{D}^{p l}\left(\eta, \dot{\bar{\varepsilon}}^{p l}\right)}=1
$$

Where $\omega_{D}$ is a state variable that increases monotonically with plastic deformation. At each increment during the analysis the incremental increase in $\omega_{D}$ is computed as:

$$
\Delta \omega_{D}=\frac{\Delta \bar{\varepsilon}^{p l}}{\bar{\varepsilon}_{D}^{p l}\left(\eta, \dot{\bar{\varepsilon}}^{p l}\right)} \geqslant 0
$$




\section{Shear criterion}

The shear criterion is a phenomenological model for predicting the onset of damage due to shear band localization. The model assumes that the equivalent plastic strain at the onset of damage, $\bar{\varepsilon}_{S}^{p l}$, is a function of the shear stress ratio $\theta_{S}$ and strain rate $\dot{\bar{\varepsilon}}^{p l}$. Here the $\theta_{S}=\left(q+k_{S} p\right) / \tau_{\max }$, where the $\tau_{\max }$ is the maximum shear stress. $k_{S}$ is a material parameter, and a typical value of for aluminum is $k_{S}=0.3$ [HOO 04]. The criterion for damage initiation is met when the following condition is satisfied:

$$
\omega_{S}=\int \frac{d \bar{\varepsilon}^{p l}}{\bar{\varepsilon}_{S}^{p l}\left(\eta_{S}, \dot{\bar{\varepsilon}}^{p l}\right)}=1
$$

Where $\omega_{S}$ is a state variable that increases monotonically with plastic deformation proportional to the incremental change in equivalent plastic strain. At each increment during the analysis the incremental increase in $\omega_{S}$ is computed as:

$$
\Delta \omega_{S}=\frac{\Delta \bar{\varepsilon}^{p l}}{\bar{\varepsilon}_{S}^{p l}\left(\eta_{S}, \dot{\bar{\varepsilon}}^{p l}\right)} \geqslant 0
$$

\section{Damage evolution}

A linear evolution of the damage variable with effective plastic displacement is applied. once the damage initiation criterion is met, the damage variable increases according to:

$$
\dot{d}=\frac{L \dot{\bar{\varepsilon}}^{p l}}{\bar{\mu}_{f}^{p l}}=\frac{\dot{\bar{\mu}}^{p l}}{\bar{\mu}_{f}^{p l}}
$$

Where the equivalent plastic displacement at failure is computed as:

$$
\bar{\mu}_{f}^{p l}={\frac{2 G_{f}}{\sigma \quad y 0}}
$$

This definition ensures that when the effective plastic displacement reaches the value $\bar{\mu}_{f}^{p l}$, the material stiffness will be fully degraded. Elements are deleted upon reaching maximum degradation.

\section{Simulation outline}

The same five particles model, as shown in FIG.5.2b, is used in this appendix. The diameter of copper particle is $20 \mu \mathrm{m}$, and the contact angle is $50^{\circ}$, and the impact velocity is $500 \mathrm{~m} / \mathrm{s}$ with a friction coefficient of 0.3 . It is hard to find the appropriate experimental data of damage initiation criterion of Copper, thus we choose the Aluminum as the substrate material due to its data are provided by the ABAQUS Example Problems 
Manuel (Section 2.1.16). All the material property used in the simulation are presented in TAB.C.2.

\begin{tabular}{|c|c|c|}
\hline Properties(Unit) & Aluminum & Copper \\
\hline Density, $\rho\left(\mathrm{kg} / \mathrm{m}^{3}\right)$ & $2.7 \times 10^{3}$ & $8.9 \times 10^{3}$ \\
Shear Modulus, $G(\mathrm{GPa})$ & 27 & 44.7 \\
Tensile strength, $\sigma_{t}(\mathrm{MPa})$ & 311 & 220 \\
Thermal conductivity, $\lambda(\mathrm{W} / \mathrm{m} \cdot \mathrm{K})$ & 237.2 & 232 \\
Specific heat, $\mathrm{c}(\mathrm{J} / \mathrm{kg} \cdot \mathrm{K})$ & 898.2 & 383 \\
Sound velocity, $\mathrm{c}_{0}(\mathrm{~m} / \mathrm{s})$ & 5386 & 3940 \\
Slope in $U_{s}$ versus $U_{p}, s$ & 1.339 & 1.489 \\
Grüneisen coefficient, $\Gamma_{0}$ & 1.97 & 2.02 \\
Yield stress, $A(\mathrm{MPa})$ & 148.4 & 90 \\
Hardening constant, $B(\mathrm{MPa})$ & 345.5 & 292 \\
Hardening exponent, $n$ & 0.183 & 0.31 \\
Strain rate constant, $C$ & 0.001 & 0.025 \\
Thermal softening exponent, $m$ & 0.895 & 1.09 \\
Melting temperature, $T_{m}(K)$ & 916 & 1356 \\
Reference temperature, $T_{0}(K)$ & 293 & 298 \\
Johnson-Cook failure model & & \\
$d_{1}$ & 0.071 & 0.54 \\
$d_{2}$ & 1.248 & 4.89 \\
$d_{3}$ & 1.142 & 3.03 \\
$d_{4}$ & 0.0147 & 0.014 \\
$d_{5}$ & 1 & 1.12 \\
\hline
\end{tabular}

Table C.2: Material properties of aluminum and copper used in the simulation.

\section{Results and discussion}

The overall deformed shape of the Copper particles and Aluminum substrate obtained by using the Progressive damage and failure method is shown in FIG.AI.1, and the deformed shape predicted by using the Dynamic failure method ([?]) is shown in FIG.AI.2 for comparison.

Firstly, the deformation shape predicted by these two damage models are totally different. The depth of crater in FIG.AI.1 is much deeper than that in FIG.AI.2; and the horizontal and vertical displacement of particles in FIG.AI.1 is totally different from that calculated by using the dynamic failure model. All the simulation results, including stress, strain, temperature, energy, etc., are dissimilar. All we could know is that the computational cost of using progressive damage failure model is lower. Moreover, the damage surface of substrate predicted by using the progressive damage failure model is smoother. 
Without comparison with the experimental results, it is hard to evaluate the capacity of each model.

In conclusion, both these two models are capable of modeling the surface erosion with appropriate material data. The experimental data is eagerly needed to validate the simulation results.

(a)

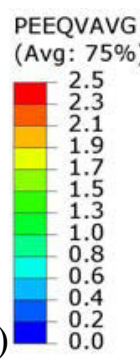

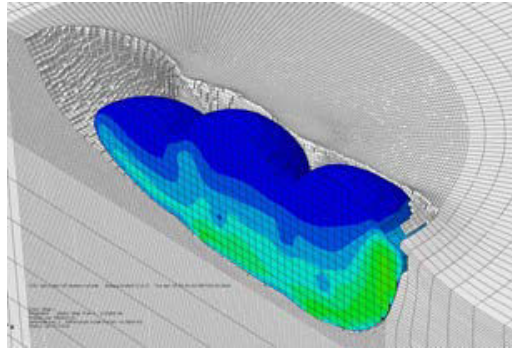

(b)

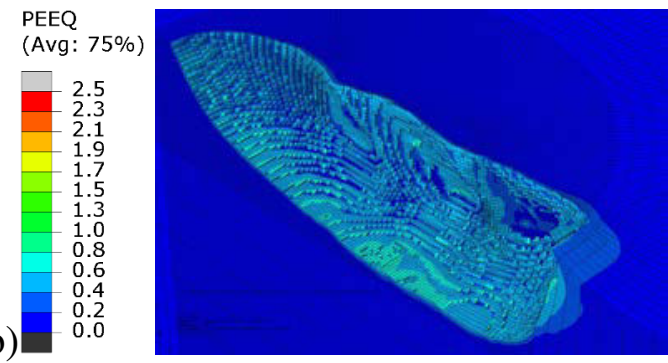

Figure AI.1: Impact of $\mathrm{Al}$ substrate by multiple $\mathrm{Cu}$ particles at $500 \mathrm{~m} / \mathrm{s}$ with contact angle of $50^{\circ}$. (a) Deformation profile of particles by using the progressive damage and failure model; (b) Deformation profile of substrate by using the progressive damage and failure model.

(a)

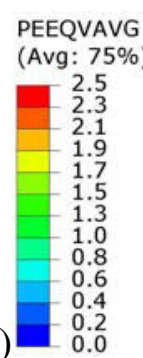

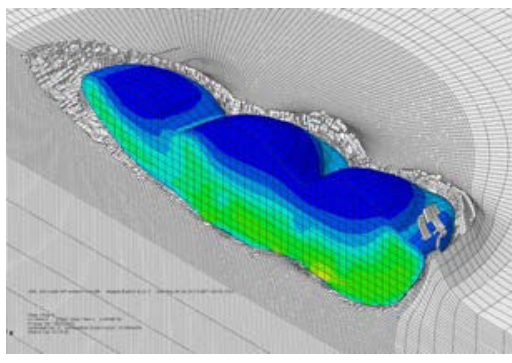

(b)

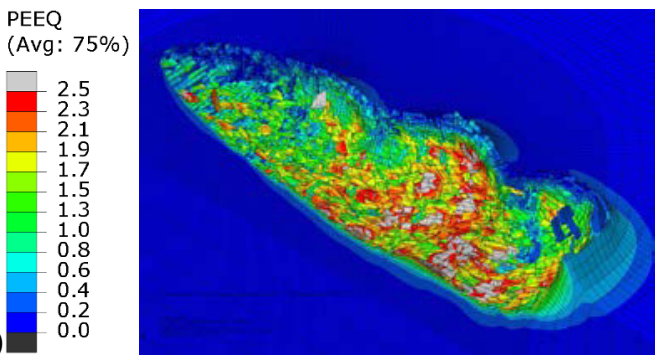

Figure AI.2: Impact of $\mathrm{Al}$ substrate by multiple $\mathrm{Cu}$ particles at $500 \mathrm{~m} / \mathrm{s}$ with contact angle of $50^{\circ}$. (a) Deformation profile of particles by using the dynamic failure model; (b) Deformation profile of substrate by using the dynamic failure model. 


\section{Appendix J: Damage initiation criterion}

\section{Ductile criterion}

Fracture Strain, Stress Triaxiality, Strain Rate

$3.3238 \mathrm{e}+01,-3.3333 \mathrm{e}+00,1.00 \mathrm{e}-04$

$3.3238 \mathrm{e}+01,-3.3333 \mathrm{e}-01,1.00 \mathrm{e}-04$

$2.3381 \mathrm{e}+01,-2.6667 \mathrm{e}-01,1.00 \mathrm{e}-04$

$1.6447 \mathrm{e}+01,-2.0000 \mathrm{e}-01,1.00 \mathrm{e}-04$

$1.1570 \mathrm{e}+01,-1.3333 \mathrm{e}-01,1.00 \mathrm{e}-04$

$8.1394 \mathrm{e}+00,-6.6667 \mathrm{e}-02,1.00 \mathrm{e}-04$

$5.7268 \mathrm{e}+00,0.0000 \mathrm{e}+00,1.00 \mathrm{e}-04$

$4.0303 \mathrm{e}+00,6.6667 \mathrm{e}-02,1.00 \mathrm{e}-04$

$2.8377 \mathrm{e}+00,1.3333 \mathrm{e}-01,1.00 \mathrm{e}-04$

$2.0000 \mathrm{e}+00,2.0000 \mathrm{e}-01,1.00 \mathrm{e}-04$

$1.4124 \mathrm{e}+00,2.6667 \mathrm{e}-01,1.00 \mathrm{e}-04$

$1.0013 \mathrm{e}+00,3.3333 \mathrm{e}-01,1.00 \mathrm{e}-04$

7.1550e-01, 4.0000e-01, 1.00e-04

$5.1920 \mathrm{e}-01,4.6667 \mathrm{e}-01,1.00 \mathrm{e}-04$

$3.8780 \mathrm{e}-01,5.3333 \mathrm{e}-01,1.00 \mathrm{e}-04$

$3.0480 \mathrm{e}-01,6.0000 \mathrm{e}-01,1.00 \mathrm{e}-04$

2.6000e-01, 6.6667e-01, 1.00e-04

$2.4758 \mathrm{e}-01,7.3010 \mathrm{e}-01,1.00 \mathrm{e}-04$

$3.0283 \mathrm{e}-01,8.5097 \mathrm{e}-01,1.00 \mathrm{e}-04$

$6.1953 \mathrm{e}-01,1.0237 \mathrm{e}+00,1.00 \mathrm{e}-04$

$1.9018 \mathrm{e}+00,1.2435 \mathrm{e}+00,1.00 \mathrm{e}-04$

$7.5608 \mathrm{e}+00,1.5058 \mathrm{e}+00,1.00 \mathrm{e}-04$

$7.5608 \mathrm{e}+00,3.3333 \mathrm{e}+00,1.00 \mathrm{e}-04$

$3.3238 \mathrm{e}+01,-3.3333 \mathrm{e}+00,1.00 \mathrm{e}-03$

$3.3238 \mathrm{e}+01,-3.3333 \mathrm{e}-01,1.00 \mathrm{e}-03$

$2.3381 \mathrm{e}+01,-2.6667 \mathrm{e}-01,1.00 \mathrm{e}-03$

$1.6447 \mathrm{e}+01,-2.0000 \mathrm{e}-01,1.00 \mathrm{e}-03$

$1.1570 \mathrm{e}+01,-1.3333 \mathrm{e}-01,1.00 \mathrm{e}-03$ 8.1394e+00, -6.6667e-02, 1.00e-03

$5.7268 \mathrm{e}+00,0.0000 \mathrm{e}+00,1.00 \mathrm{e}-03$

$4.0303 \mathrm{e}+00,6.6667 \mathrm{e}-02,1.00 \mathrm{e}-03$

$2.8377 \mathrm{e}+00,1.3333 \mathrm{e}-01,1.00 \mathrm{e}-03$

$2.0000 \mathrm{e}+00,2.0000 \mathrm{e}-01,1.00 \mathrm{e}-03$

$1.4124 \mathrm{e}+00,2.6667 \mathrm{e}-01,1.00 \mathrm{e}-03$

$1.0013 \mathrm{e}+00,3.3333 \mathrm{e}-01,1.00 \mathrm{e}-03$

$7.1550 \mathrm{e}-01,4.0000 \mathrm{e}-01,1.00 \mathrm{e}-03$

$5.1920 \mathrm{e}-01,4.6667 \mathrm{e}-01,1.00 \mathrm{e}-03$

$3.8780 \mathrm{e}-01,5.3333 \mathrm{e}-01,1.00 \mathrm{e}-03$

$3.0480 \mathrm{e}-01,6.0000 \mathrm{e}-01,1.00 \mathrm{e}-03$

$2.6000 \mathrm{e}-01,6.6667 \mathrm{e}-01,1.00 \mathrm{e}-03$

$2.4758 \mathrm{e}-01,7.3010 \mathrm{e}-01,1.00 \mathrm{e}-03$

$3.0283 \mathrm{e}-01,8.5097 \mathrm{e}-01,1.00 \mathrm{e}-03$

$6.1953 \mathrm{e}-01,1.0237 \mathrm{e}+00,1.00 \mathrm{e}-03$

$1.9018 \mathrm{e}+00,1.2435 \mathrm{e}+00,1.00 \mathrm{e}-03$

$7.5608 \mathrm{e}+00,1.5058 \mathrm{e}+00,1.00 \mathrm{e}-03$

$7.5608 \mathrm{e}+00,3.3333 \mathrm{e}+00,1.00 \mathrm{e}-03$

$8.4132 \mathrm{e}+01,-3.3333 \mathrm{e}+00,2.50 \mathrm{e}+02$

$8.4132 \mathrm{e}+01,-3.3333 \mathrm{e}-01,2.50 \mathrm{e}+02$

$4.7327 \mathrm{e}+01,-2.6667 \mathrm{e}-01,2.50 \mathrm{e}+02$

$2.6621 \mathrm{e}+01,-2.0000 \mathrm{e}-01,2.50 \mathrm{e}+02$

$1.4974 \mathrm{e}+01,-1.3333 \mathrm{e}-01,2.50 \mathrm{e}+02$

$8.4238 \mathrm{e}+00,-6.6667 \mathrm{e}-02,2.50 \mathrm{e}+02$

$4.7393 \mathrm{e}+00,0.0000 \mathrm{e}+00,2.50 \mathrm{e}+02$

$2.6675 \mathrm{e}+00,6.6667 \mathrm{e}-02,2.50 \mathrm{e}+02$

$1.5034 \mathrm{e}+00,1.3333 \mathrm{e}-01,2.50 \mathrm{e}+02$

$8.5084 \mathrm{e}-01,2.0000 \mathrm{e}-01,2.50 \mathrm{e}+02$ 
Appendix J: Damage initiation criterion

$4.8779 \mathrm{e}-01,2.6667 \mathrm{e}-01,2.50 \mathrm{e}+02$

$2.9076 \mathrm{e}-01,3.3333 \mathrm{e}-01,2.50 \mathrm{e}+02$

$1.9263 \mathrm{e}-01,4.0000 \mathrm{e}-01,2.50 \mathrm{e}+02$

$1.6006 \mathrm{e}-01,4.6667 \mathrm{e}-01,2.50 \mathrm{e}+02$

$1.8195 \mathrm{e}-01,5.3333 \mathrm{e}-01,2.50 \mathrm{e}+02$

$2.6576 \mathrm{e}-01,6.0000 \mathrm{e}-01,2.50 \mathrm{e}+02$

$4.4001 \mathrm{e}-01,6.6667 \mathrm{e}-01,2.50 \mathrm{e}+02$

$7.4341 \mathrm{e}-01,7.3010 \mathrm{e}-01,2.50 \mathrm{e}+02$

$2.0883 \mathrm{e}+00,8.5097 \mathrm{e}-01,2.50 \mathrm{e}+02$

$9.2599 \mathrm{e}+00,1.0237 \mathrm{e}+00,2.50 \mathrm{e}+02$

$6.1718 \mathrm{e}+01,1.2435 \mathrm{e}+00,2.50 \mathrm{e}+02$

$5.9367 \mathrm{e}+02,1.5058 \mathrm{e}+00,2.50 \mathrm{e}+02$

$5.9367 \mathrm{e}+02,3.3333 \mathrm{e}+00,2.50 \mathrm{e}+02$

$8.4132 \mathrm{e}+01,-3.3333 \mathrm{e}+00,1.00 \mathrm{e}+03$

$8.4132 \mathrm{e}+01,-3.3333 \mathrm{e}-01,1.00 \mathrm{e}+03$

$4.7327 \mathrm{e}+01,-2.6667 \mathrm{e}-01,1.00 \mathrm{e}+03$

$2.6621 \mathrm{e}+01,-2.0000 \mathrm{e}-01,1.00 \mathrm{e}+03$

$1.4974 \mathrm{e}+01,-1.3333 \mathrm{e}-01,1.00 \mathrm{e}+03$

$8.4238 \mathrm{e}+00,-6.6667 \mathrm{e}-02,1.00 \mathrm{e}+03$
$4.7393 \mathrm{e}+00,0.0000 \mathrm{e}+00,1.00 \mathrm{e}+03$

$2.6675 \mathrm{e}+00,6.6667 \mathrm{e}-02,1.00 \mathrm{e}+03$

$1.5034 \mathrm{e}+00,1.3333 \mathrm{e}-01,1.00 \mathrm{e}+03$

$8.5084 \mathrm{e}-01,2.0000 \mathrm{e}-01,1.00 \mathrm{e}+03$

$4.8779 \mathrm{e}-01,2.6667 \mathrm{e}-01,1.00 \mathrm{e}+03$

$2.9076 \mathrm{e}-01,3.3333 \mathrm{e}-01,1.00 \mathrm{e}+03$

$1.9263 \mathrm{e}-01,4.0000 \mathrm{e}-01,1.00 \mathrm{e}+03$

$1.6006 \mathrm{e}-01,4.6667 \mathrm{e}-01,1.00 \mathrm{e}+03$

$1.8195 \mathrm{e}-01,5.3333 \mathrm{e}-01,1.00 \mathrm{e}+03$

$2.6576 \mathrm{e}-01,6.0000 \mathrm{e}-01,1.00 \mathrm{e}+03$

$4.4001 \mathrm{e}-01,6.6667 \mathrm{e}-01,1.00 \mathrm{e}+03$

$7.4341 \mathrm{e}-01,7.3010 \mathrm{e}-01,1.00 \mathrm{e}+03$

$2.0883 \mathrm{e}+00,8.5097 \mathrm{e}-01,1.00 \mathrm{e}+03$

$9.2599 \mathrm{e}+00,1.0237 \mathrm{e}+00,1.00 \mathrm{e}+03$

$6.1718 \mathrm{e}+01,1.2435 \mathrm{e}+00,1.00 \mathrm{e}+03$

$5.9367 \mathrm{e}+02,1.5058 \mathrm{e}+00,1.00 \mathrm{e}+03$

$5.9367 \mathrm{e}+02,3.3333 \mathrm{e}+00,1.00 \mathrm{e}+03$

\section{Shear criterion}

Fracture Strain, Stress Stress Ratio, Strain Rate

$2.7610 \mathrm{e}-01,-1.0000 \mathrm{e}+01,1.00 \mathrm{e}-04$

$2.7610 \mathrm{e}-01,1.4236 \mathrm{e}+00,1.00 \mathrm{e}-04$

$2.6130 \mathrm{e}-01,1.4625 \mathrm{e}+00,1.00 \mathrm{e}-04$

$2.5300 \mathrm{e}-01,1.5013 \mathrm{e}+00,1.00 \mathrm{e}-04$

$2.5100 \mathrm{e}-01,1.5401 \mathrm{e}+00,1.00 \mathrm{e}-04$

$2.5510 \mathrm{e}-01,1.5789 \mathrm{e}+00,1.00 \mathrm{e}-04$

$2.6560 \mathrm{e}-01,1.6177 \mathrm{e}+00,1.00 \mathrm{e}-04$

$2.8250 \mathrm{e}-01,1.6566 \mathrm{e}+00,1.00 \mathrm{e}-04$

$3.0650 \mathrm{e}-01,1.6954 \mathrm{e}+00,1.00 \mathrm{e}-04$

$3.3790 \mathrm{e}-01,1.7342 \mathrm{e}+00,1.00 \mathrm{e}-04$

$3.7780 \mathrm{e}-01,1.7730 \mathrm{e}+00,1.00 \mathrm{e}-04$

$4.2690 \mathrm{e}-01,1.8118 \mathrm{e}+00,1.00 \mathrm{e}-04$

$4.8650 \mathrm{e}-01,1.8506 \mathrm{e}+00,1.00 \mathrm{e}-04$

$5.5810 \mathrm{e}-01,1.8895 \mathrm{e}+00,1.00 \mathrm{e}-04$

$6.4350 \mathrm{e}-01,1.9283 \mathrm{e}+00,1.00 \mathrm{e}-04$

$7.4480 \mathrm{e}-01,1.9671 \mathrm{e}+00,1.00 \mathrm{e}-04$

$8.6440 \mathrm{e}-01,2.0059 \mathrm{e}+00,1.00 \mathrm{e}-04$
$1.0053 \mathrm{e}+00,2.0447 \mathrm{e}+00,1.00 \mathrm{e}-04$

$1.1710 \mathrm{e}+00,2.0835 \mathrm{e}+00,1.00 \mathrm{e}-04$

$1.3655 \mathrm{e}+00,2.1224 \mathrm{e}+00,1.00 \mathrm{e}-04$

$1.5937 \mathrm{e}+00,2.1612 \mathrm{e}+00,1.00 \mathrm{e}-04$

$1.8611 \mathrm{e}+00,2.2000 \mathrm{e}+00,1.00 \mathrm{e}-04$

$1.8611 \mathrm{e}+00,1.0000 \mathrm{e}+01,1.00 \mathrm{e}-04$

$2.7610 \mathrm{e}-01,-1.0000 \mathrm{e}+01,1.00 \mathrm{e}-03$

$2.7610 \mathrm{e}-01,1.4236 \mathrm{e}+00,1.00 \mathrm{e}-03$

$2.6130 \mathrm{e}-01,1.4625 \mathrm{e}+00,1.00 \mathrm{e}-03$

$2.5300 \mathrm{e}-01,1.5013 \mathrm{e}+00,1.00 \mathrm{e}-03$

$2.5100 \mathrm{e}-01,1.5401 \mathrm{e}+00,1.00 \mathrm{e}-03$

$2.5510 \mathrm{e}-01,1.5789 \mathrm{e}+00,1.00 \mathrm{e}-03$

$2.6560 \mathrm{e}-01,1.6177 \mathrm{e}+00,1.00 \mathrm{e}-03$

$2.8250 \mathrm{e}-01,1.6566 \mathrm{e}+00,1.00 \mathrm{e}-03$

$3.0650 \mathrm{e}-01,1.6954 \mathrm{e}+00,1.00 \mathrm{e}-03$

$3.3790 \mathrm{e}-01,1.7342 \mathrm{e}+00,1.00 \mathrm{e}-03$

$3.7780 \mathrm{e}-01,1.7730 \mathrm{e}+00,1.00 \mathrm{e}-03$ 
$4.2690 \mathrm{e}-01,1.8118 \mathrm{e}+00,1.00 \mathrm{e}-03$ $4.8650 \mathrm{e}-01,1.8506 \mathrm{e}+00,1.00 \mathrm{e}-03$ $5.5810 \mathrm{e}-01,1.8895 \mathrm{e}+00,1.00 \mathrm{e}-03$ $6.4350 \mathrm{e}-01,1.9283 \mathrm{e}+00,1.00 \mathrm{e}-03$ $7.4480 \mathrm{e}-01,1.9671 \mathrm{e}+00,1.00 \mathrm{e}-03$ $8.6440 \mathrm{e}-01,2.0059 \mathrm{e}+00,1.00 \mathrm{e}-03$ $1.0053 \mathrm{e}+00,2.0447 \mathrm{e}+00,1.00 \mathrm{e}-03$ $1.1710 \mathrm{e}+00,2.0835 \mathrm{e}+00,1.00 \mathrm{e}-03$ $1.3655 \mathrm{e}+00,2.1224 \mathrm{e}+00,1.00 \mathrm{e}-03$ $1.5937 \mathrm{e}+00,2.1612 \mathrm{e}+00,1.00 \mathrm{e}-03$ $1.8611 \mathrm{e}+00,2.2000 \mathrm{e}+00,1.00 \mathrm{e}-03$ $1.8611 \mathrm{e}+00,1.0000 \mathrm{e}+01,1.00 \mathrm{e}-03$ $3.3382 \mathrm{e}-01,-1.0000 \mathrm{e}+01,2.50 \mathrm{e}+02$ $3.3382 \mathrm{e}-01,1.4236 \mathrm{e}+00,2.50 \mathrm{e}+02$ $3.3361 \mathrm{e}-01,1.4625 \mathrm{e}+00,2.50 \mathrm{e}+02$ $3.3552 \mathrm{e}-01,1.5013 \mathrm{e}+00,2.50 \mathrm{e}+02$ $3.3955 \mathrm{e}-01,1.5401 \mathrm{e}+00,2.50 \mathrm{e}+02$ $3.4572 \mathrm{e}-01,1.5789 \mathrm{e}+00,2.50 \mathrm{e}+02$ $3.5409 \mathrm{e}-01,1.6177 \mathrm{e}+00,2.50 \mathrm{e}+02$ $3.6473 \mathrm{e}-01,1.6566 \mathrm{e}+00,2.50 \mathrm{e}+02$ $3.7765 \mathrm{e}-01,1.6954 \mathrm{e}+00,2.50 \mathrm{e}+02$ $3.9297 \mathrm{e}-01,1.7342 \mathrm{e}+00,2.50 \mathrm{e}+02$ $4.1077 \mathrm{e}-01,1.7730 \mathrm{e}+00,2.50 \mathrm{e}+02$ $4.3117 \mathrm{e}-01,1.8118 \mathrm{e}+00,2.50 \mathrm{e}+02$ $4.5430 \mathrm{e}-01,1.8506 \mathrm{e}+00,2.50 \mathrm{e}+02$ $4.8038 \mathrm{e}-01,1.8895 \mathrm{e}+00,2.50 \mathrm{e}+02$ $5.0943 \mathrm{e}-01,1.9283 \mathrm{e}+00,2.50 \mathrm{e}+02$ $5.4171 \mathrm{e}-01,1.9671 \mathrm{e}+00,2.50 \mathrm{e}+02$ $5.7742 \mathrm{e}-01,2.0059 \mathrm{e}+00,2.50 \mathrm{e}+02$ $6.1678 \mathrm{e}-01,2.0447 \mathrm{e}+00,2.50 \mathrm{e}+02$
$6.6005 \mathrm{e}-01,2.0835 \mathrm{e}+00,2.50 \mathrm{e}+02$ 7.0762e-01, 2.1224e+00, 2.50e+02 $7.5956 \mathrm{e}-01,2.1612 \mathrm{e}+00,2.50 \mathrm{e}+02$ $8.1630 \mathrm{e}-01,2.2000 \mathrm{e}+00,2.50 \mathrm{e}+02$ $8.1630 \mathrm{e}-01,1.0000 \mathrm{e}+01,2.50 \mathrm{e}+02$ $3.3382 \mathrm{e}-01,-1.0000 \mathrm{e}+01,1.00 \mathrm{e}+03$ $3.3382 \mathrm{e}-01,1.4236 \mathrm{e}+00,1.00 \mathrm{e}+03$ $3.3361 \mathrm{e}-01,1.4625 \mathrm{e}+00,1.00 \mathrm{e}+03$ $3.3552 \mathrm{e}-01,1.5013 \mathrm{e}+00,1.00 \mathrm{e}+03$ $3.3955 \mathrm{e}-01,1.5401 \mathrm{e}+00,1.00 \mathrm{e}+03$ $3.4572 \mathrm{e}-01,1.5789 \mathrm{e}+00,1.00 \mathrm{e}+03$ $3.5409 \mathrm{e}-01,1.6177 \mathrm{e}+00,1.00 \mathrm{e}+03$ $3.6473 \mathrm{e}-01,1.6566 \mathrm{e}+00,1.00 \mathrm{e}+03$ $3.7765 \mathrm{e}-01,1.6954 \mathrm{e}+00,1.00 \mathrm{e}+03$ $3.9297 \mathrm{e}-01,1.7342 \mathrm{e}+00,1.00 \mathrm{e}+03$ $4.1077 \mathrm{e}-01,1.7730 \mathrm{e}+00,1.00 \mathrm{e}+03$ $4.3117 \mathrm{e}-01,1.8118 \mathrm{e}+00,1.00 \mathrm{e}+03$ $4.5430 \mathrm{e}-01,1.8506 \mathrm{e}+00,1.00 \mathrm{e}+03$ $4.8038 \mathrm{e}-01,1.8895 \mathrm{e}+00,1.00 \mathrm{e}+03$ $5.0943 \mathrm{e}-01,1.9283 \mathrm{e}+00,1.00 \mathrm{e}+03$ $5.4171 \mathrm{e}-01,1.9671 \mathrm{e}+00,1.00 \mathrm{e}+03$ $5.7742 \mathrm{e}-01,2.0059 \mathrm{e}+00,1.00 \mathrm{e}+03$ $6.1678 \mathrm{e}-01,2.0447 \mathrm{e}+00,1.00 \mathrm{e}+03$ $6.6005 \mathrm{e}-01,2.0835 \mathrm{e}+00,1.00 \mathrm{e}+03$ $7.0762 \mathrm{e}-01,2.1224 \mathrm{e}+00,1.00 \mathrm{e}+03$ $7.5956 \mathrm{e}-01,2.1612 \mathrm{e}+00,1.00 \mathrm{e}+03$ $8.1630 \mathrm{e}-01,2.2000 \mathrm{e}+00,1.00 \mathrm{e}+03$ $8.1630 \mathrm{e}-01,1.0000 \mathrm{e}+01,1.00 \mathrm{e}+03$ 


\section{Bibliography}

[AKB 12] Akbarzadeh E., Elsaadawy E., Sherik A., Spelt J., Papini M.

The solid particle erosion of 12 metals using magnetite erodent. Wear, vol. 282-283, 2012, p. 40-51.

[AQU 01] AQUaro D., Fontani E.

Erosion of Ductile and Brittle Materials. Meccanica, vol. 36, $\mathrm{n}^{\circ}$ 6, 2001, p. 651-661.

[ASS 03] Assadi H., Gartner F., Stoltenhoff T., Kreye H.

Bonding mechanism in cold gas spraying. Acta Materialia, vol. 51, $\mathrm{n}^{\mathrm{o}}$ 15, 2003, p. $4379-4394$.

[BAE 08] Bae G., Xiong Y., Kumar S., Kang K., Lee C.

General aspects of interface bonding in kinetic sprayed coatings. Acta Materialia, vol. $56, \mathrm{n}^{\mathrm{o}} 17,2008$, p. $4858-4868$.

[BAS 08] BASSIM M. N., ODESHI A.

Shear strain localisation and fracture in high strength structural materials. Archives of Materials Science, vol. 70, 2008, p. 69-74.

[BEC 81] BECKMANn G., GotZMAnN J.

Analytical model of the blast wear intensity of metals based on a general arrangement for abrasive wear. Wear, vol. 73, no 2, 1981, p. 325-353.

[BEL 13] BelytschKo T., LiU W., Moran B., ElKhodary K.

Nonlinear Finite Elements for Continua and Structures. Wiley Desktop Editions Wiley, 2013.

[BEN 10] BenZerga A. A., Leblond J. B.

Ductile fracture by void growth to coalescence. Advances in Applied Mechanics, vol. 44, 2010, p. 169-305.

[BIT 63] BITTER J.

A study of erosion phenomena part I. Wear, vol. 6, $\mathrm{n}^{\mathrm{o}}$ 1, 1963, p. 5-21.

[BUZ 13] BuZYurkin A. E., Kraus E. I., LuKyanov Y. L.

Study of the conditions of fracture at explosive compaction of powders. Fracture and Structural Integrity, vol. 24, 2013, p. 102-111.

[CAL 11] CALEYRON F.

Simulation numérique par la méthode SPH de fuites de fluide consécutives à la déchirure d? un réservoir sous impact. PhD Thesis, INSA de Lyon, Lyon, FR, October 2011. 
[CAL 13] Caleyron F., Combescure A., Faucher V., Potapov S. $\{\mathrm{SPH}\}$ modeling of fluid-"solid interaction for dynamic failure analysis of fluid-filled thin shells. Journal of Fluids and Structures, vol. 39, n 0, 2013, p. 126-153.

[CHA 05] Champagne V. J., Helfritch D., Leyman P., Grendahl S., Klotz B. Interface material mixing formed by the deposition of copper on aluminum by means of the cold spray process. Journal of Thermal Spray Technology, vol. 14, $\mathrm{n}^{\mathrm{0}}$ 3, 2005, p. 330-334.

[CHA 07] CHAMPAGNE V.

The Cold Spray Materials Deposition Process: Fundamentals and Applications. Series in Metals and Surface Engineering Series Woodhead Publishing Limited, 2007.

[CHA 11] Chavan N. M., Ramakrishna M., Phani P. S., Rao D. S., SunDARARAJAN G.

The influence of process parameters and heat treatment on the properties of cold sprayed silver coatings. Surface and Coatings Technology, vol. 205, $\mathrm{n}^{\mathrm{O}}$ 20, 2011, p. $4798-4807$.

[CHE 03] CHEN Q., LI D.

Computer simulation of solid particle erosion. Wear, vol. 254, n ${ }^{\mathrm{o}} 3-4,2003$, p. 203210.

[COM 08] Combescure A., Maurel B., Potapov S.

Modelling dynamic fracture of thin shells filled with fluid: a fully SPH model. Mécanique Industries, vol. 9, 2008, p. 167-174.

[DAS11] DASSAULT Systemes, Abaqus Analysis User's Manue, version 6.11 édition, 2011.

[ELT 05] ElTobgy M., Ng E., Elbestawi M.

Finite element modeling of erosive wear. International Journal of Machine Tools and Manufacture, vol. 45, $\mathrm{n}^{\mathrm{O}}$ 11, 2005, p. 1337-1346.

[FAI 00] FAIK S., WiTTEMAN H.

Modeling of Impact Dynamics: A Literature Survey. International ADAMS User Conference, Toronto, Canada, 2000.

[FIN 60] FINNIE I.

Erosion of surfaces by solid particles. Wear, vol. 3, no 2, 1960, p. 87-103.

[GHE 11] Ghelichi R., Bagherifard S., Guagliano M., Verani M. Numerical simulation of cold spray coating. Surface and Coatings Technology, vol. $205, \mathrm{n}^{\text {o }} 23-24,2011$, p. 5294-5301.

[GHE 12] Ghelichi R., MacDonald D., BAgherifard S., JAHED H., Guagliano M., Jodoin B.

Microstructure and fatigue behavior of cold spray coated A15052. Acta Materialia, vol. $60, \mathrm{n}^{\mathrm{o}} 19,2012$, p. 6555-6561.

[GRI 04] GRIFFIn D., DAADbin A., DATTA S.

The development of a three-dimensional finite element model for solid particle erosion on an alumina scale/MA956 substrate. Wear, vol. 256, n 9-10, 2004, p. 900-906. 
[GRU 03] Grujicic M., Saylor J., Beasley D., DeRosset W., Helfritch D.

Computational analysis of the interfacial bonding between feed-powder particles and the substrate in the cold-gas dynamic-spray process. Applied Surface Science, vol. 219, no 3-4, 2003, p. 211-227.

[GRU 04] Grujicic M., Zhao C., DeRosset W., Helfritch D.

Adiabatic shear instability based mechanism for particles/substrate bonding in the cold-gas dynamic-spray process. Materials \&amp; Design, vol. 25, $\mathrm{n}^{\mathrm{o}}$ 8, 2004, p. $681-688$.

[GUE 09] Guetta S., Berger M., Borit F., Guipont V., Jeandin M., Boustie M., ICHikawa Y., SAKaguchi K., Ogawa K.

Influence of Particle Velocity on Adhesion of Cold-Sprayed Splats. Journal of Thermal Spray Technology, vol. 18, 2009, p. 331-342.

[GUI 11] Guida M., Marulo F., Meo M., Grimaldi A., Olivares G.

SPH - Lagrangian study of bird impact on leading edge wing. Composite Structures, vol. 93, no 3, 2011, p. 1060-1071.

[HEN 13] Henryk P., Lidia L.-D., MARIUSz P.

Microstructure and Phase Constitution Near the Interface of Explosively Welded Aluminum/Copper Plates. Metallurgical and Materials Transactions A, vol. 44, $\mathrm{n}^{\mathrm{O}} 8$, 2013, p. 3836-3851.

[HIL 76] Hillerborg A., Modéer M., Petersson P.-E.

Analysis of crack formation and crack growth in concrete by means of fracture mechanics and finite elements. Cement and Concrete Research, vol. 6, $\mathrm{n}^{\mathrm{o}}$ 6, 1976, p. 773-781.

[HOO 04] Hooputra H., Gese H., Dell H., Werner H.

A comprehensive failure model for crashworthiness simulation of aluminium extrusions. International Journal of Crashworthiness, vol. 9, $\mathrm{n}^{\circ}$ 5, 2004, p. 449-464.

[HUT 76] Hutchings I., Winter R., Field J.

Solid Particle Erosion of Metals: The Removal of Surface Material by Spherical Projectiles. Proceedings of the Royal Society of London. Series A, Mathematical and Physical Sciences, vol. 348 The Royal Society, 1976, p. 379-392.

[JOH 83] Johnson G. R., COOK W. H.

A Constitutive Model and Data for Metals Subjected to Large Strains, High Strain Rates and High Temperatures. Proceeding of the 7th International Symposium on Ballistics, Hague, Netherlands, 1983 p. 541-547.

[JOH 85] JOHnSON G. R., COOK W. H.

Fracture characteristics of three metals subjected to various strains, strain rates, temperatures and pressures. Engineering Fracture Mechanics, vol. 21, n ${ }^{\circ}$ 1, 1985, p. 31-48.

[KIN 08] King P., ZAhiRi S., JAHEDi M.

Focused ion beam micro-dissection of cold-sprayed particles. Acta Materialia, vol. 56, $n^{\mathrm{o}} 19,2008$, p. 5617-5626. 
[KIN 10] King P. C., BAE G., ZahiRi S. H., Jahedi M., LeE C.

An Experimental and Finite Element Study of Cold Spray Copper Impact onto Two Aluminum Substrates. Journal of Thermal Spray Technology, vol. 19, $\mathrm{n}^{\mathrm{o}}$ 3, 2010, p. 620-634.

[KLE 07] KLEIS I., KULU P.

Solid Particle Erosion: Occurrence, Prediction and Control. Springer, 2007.

[KLI 05] Klinkov S. V., Kosarev V. F., Rein M.

Cold spray deposition: Significance of particle impact phenomena. Aerospace Science and Technology, vol. 9, $\mathrm{n}^{\mathrm{o}}$ 7, 2005, p. 582-591.

[LEE 04] LeE H. Y., YU Y. H., LeE Y. C., Hong Y. P., Ko K. H. Interfacial studies between cold sprayed WO3 Y2O3 films and Si substrate. Applied Surface Science, vol. 227, $\mathrm{n}^{\mathrm{O}}$ 1-4, 2004, p. 244-249.

[LEE 07] LEE J., ShIN S., KiM H., LEE C.

Effect of gas temperature on critical velocity and deposition characteristics in kinetic spraying. Applied Surface Science, vol. 253, n ${ }^{\circ}$ 7, 2007, p. 3512-3520.

[LEE 11] Lee M.-W., PARK J.-J., Kim D.-Y., Yoon S. S., Kim H.-Y., James S. C., ChandRA S., COYLE T.

Numerical Studies on the Effects of Stagnation Pressure and Temperature on Supersonic Flow Characteristics in Cold Spray Applications. Journal of Thermal Spray Technology, vol. 20, 2011, p. 1085-1097.

[LI 05] Li C., Li W.-Y., WANG Y., YANG G., FuKanuma H.

A theoretical model for prediction of deposition efficiency in cold spraying. Thin Solid Films, vol. 489, $\mathrm{n}^{\mathrm{o}} 1-2,2005$, p. 79-85.

[Li 06] Li W., Liao H., Li C., Li G., Coddet C., Wang X.

On high velocity impact of micro-sized metallic particles in cold spraying. Applied Surface Science, vol. 253, no 5, 2006, p. 2852-2862.

[LI 07a] LI G., WANG X., LI W.

Effect of different incidence angles on bonding performance in cold spraying. Transactions of Nonferrous Metals Society of China, vol. 17, $\mathrm{n}^{\mathrm{0}}$ 1, 2007, p. 116-121.

[LI 07b] Li W., Liao H., Li C., BANG H.-S., Coddet C.

Numerical simulation of deformation behavior of $\mathrm{Al}$ particles impacting on $\mathrm{Al}$ substrate and effect of surface oxide films on interfacial bonding in cold spraying. Applied Surface Science, vol. 253, n ${ }^{\mathrm{O}}$ 11, 2007, p. 5084-5091.

[LI 09a] LI W., GAO W.

Some aspects on 3D numerical modeling of high velocity impact of particles in cold spraying by explicit finite element analysis. Applied Surface Science, vol. 255, $\mathrm{n}^{\mathrm{O}} 18$, 2009, p. 7878-7892.

[LI 09b] Li W., Zhang C., Li C., LiaO H.

Modeling Aspects of High Velocity Impact of Particles in Cold Spraying by Explicit Finite Element Analysis. Journal of Thermal Spray Technology, vol. 18, 2009, p. 921933. 
[LI 10a] LI W., LI C., YANG G.

Effect of impact-induced melting on interface microstructure and bonding of cold sprayed zinc coating. Applied Surface Science, vol. 257, no 5, 2010, p. 1516-1523.

[LI 10b] Li W., Yin S., WANG X.

Numerical investigations of the effect of oblique impact on particle deformation in cold spraying by the SPH method. Applied Surface Science, vol. 256, n ${ }^{0}$ 12, 2010, p. $3725-3734$.

[LUZ 11] LUZIN V., SPENCER K., ZHANG M.

Residual stress and thermo-mechanical properties of cold spray metal coatings. Acta Materialia, vol. 59, $\mathrm{n}^{\mathrm{O}}$ 3, 2011, p. 1259-1270.

[MAN 11a] Manap A., OKabe T., Ogawa K.

Computer simulation of cold sprayed deposition using smoothed particle hydrodynamics. Procedia Engineering, vol. 10, 2011, p. 1145-1150.

[MAN 11b] ManaP A., OGaWA K., OKABE T.

Numerical Analysis of Interfacial Bonding of Aluminum Powder Particle and Aluminum Substrate by Cold Spray Technique using the SPH Method. Proceedings of the JSME/ASME 2011 ICMP2011, Corvallis,Oregon,USA, 2011.

[MAN 11c] MANAP A. N. A.

Mechanical analysis of interfacial bonding of cold sprayed metallic particles usin smoothed particle hydrodynamics method. PhD Thesis, Tohoku University, Sendai, JP, July 2011.

[MAN 12] MANAP A., OGAWA K., OKaBe T.

Numerical Analysis of Interfacial Bonding of Al-Si Particle and Mild Steel Substrate by Cold Spray Technique Using the SPH Method. Journal of Solid Mechanics and Materials Engineering, vol. 6, 2012, p. 241-250.

[MAR 06] Marx S., PAul A., KÖHLER A., HütTl G.

Cold spraying: Innovative layers for new applications. Journal of Thermal Spray Technology, vol. $15, \mathrm{n}^{\mathrm{O}}$ 2, 2006, p. 177-183.

[MAU 08a] Maurel B., Combescure A.

An SPH shell formulation for plasticity and fracture analysis in explicit dynamics. International Journal for Numerical Methods in Engineering, vol. 76, $\mathrm{n}^{\mathrm{o}}$ 7, 2008, p. 949-971.

[MAU 08b] MAUREL B.

Modelisation par la méthode SPH de l'impact d'un reservoir rempli de fluide. $\mathrm{PhD}$ Thesis, INSA de Lyon, Lyon, FR, January 2008.

[MCC 00] McCune R., Donlon W., Popoola O., Cartwright E.

Characterization of copper layers produced by cold gas-dynamic spraying. Journal of Thermal Spray Technology, vol. 9, $\mathrm{n}^{\mathrm{o}}$ 1, 2000, p. 73-82.

[MOL 02] MOLINARi J., ORTIZ M.

A study of solid-particle erosion of metallic targets. International Journal of Impact Engineering, vol. 27, $\mathrm{n}^{\mathrm{o}} 4,2002$, p. 347-358. 
[OGA 08] Ogawa K., Ito K., IChimura K., IChikawa Y., Ohno S., Onda N. Characterization of Low-Pressure Cold-Sprayed Aluminum Coatings. Journal of Thermal Spray Technology, vol. 17, 2008, p. 728-735.

[OKA 93] OKa Y., Matsumura M., Kawabata T.

Relationship between surface hardness and erosion damage caused by solid particle impact. Wear, vol. 162-164, Part B, 1993, p. 688-695.

[OKA 03] OKA Y. I., NAGAHASHI K.

Measurements of plastic strain around indentations caused by the impact of round and angular particles, and the origin of erosion. Wear, vol. 254, $\mathrm{n}^{\mathrm{o}} 12,2003$, p. 12671275 .

[OKA 05a] OKa Y., OKamura K., Yoshida T.

Practical estimation of erosion damage caused by solid particle impact: Part 1: Effects of impact parameters on a predictive equation. Wear, vol. 259, $\mathrm{n}^{\mathrm{o}} 1-6,2005$, p. 95101.

[OKA 05b] OKA Y., YoshidA T.

Practical estimation of erosion damage caused by solid particle impact: Part 2: Mechanical properties of materials directly associated with erosion damage. Wear, vol. 259, no 1-6, 2005, p. 102-109.

[OKA 05c] OKa Y. I., Nagahashi K., Ishit Y., KobaYashi Y., Tsumura T.

Damage behaviour of metallic materials caused by subsonic to hypervelocity particle impact. Wear, vol. 258, $\mathrm{n}^{\mathrm{o}} 1-4,2005$, p. 100-106.

[OKA 09] OKa Y., Mihara S., Yoshida T.

Impact-angle dependence and estimation of erosion damage to ceramic materials caused by solid particle impact. Wear, vol. 267, no 1-4, 2009, p. 129-135.

[PAP 06] Papyrin A., Kosarev V., Klinkov S., Alkhimov A., Fomin V. Cold Spray Technology. Elsevier Science, 2006.

[PAT 08] Pattison J., Celotto S., Khan A., O’Neill W.

Standoff distance and bow shock phenomena in the Cold Spray process. Surface and Coatings Technology, vol. 202, $\mathrm{n}^{\mathrm{O}}$ 8, 2008, p. 1443-1454.

[QIU 11] QIU G., GRABE S. H. J.

Application of a Coupled Eulerian-Lagrangian approach on geomechanical problems involving large deformations. Computers and Geotechnics, vol. 38, $\mathrm{n}^{\mathrm{O}} 1,2011$, p. 3039.

[RAM 04] RAMSDALE R.

. « Engineer's Handbook». www.engineershandbook.com/Tables/frictioncoefficients.htm, 2004.

[RUF 79] RUFF A. W., WiEdERHORN S. M.

Erosion by Solid Particle Impact. Interim Report no NBSIR78-1575, January 1979, National Measurement Laboratory, Center for Materials Science and National Bureau of Standards Washington D.C. 20234. 
[SAM 07] SAMAREH B., Dolatabadi A.

A Three-Dimensional Analysis of the Cold Spray Process: The Effects of Substrate Location and Shape. Journal of Thermal Spray Technology, vol. 16, 2007, p. 634642.

[SHI 99] Shimizu K., Noguchi T., Seitoh H., Muranaka E.

FEM analysis of the dependency on impact angle during erosive wear. Wear, vol. 233235,1999 , p. 157-159.

[ShI 01] Shimizu K., Noguchi T., Seitoh H., OKada M., Matsubara Y. FEM analysis of erosive wear. Wear, vol. 250, $\mathrm{n}^{\mathrm{o}}$ 1-12, 2001, p. 779-784.

[SHR 11] SHROTA A., BAKER M.

Inverse identification of Johnson-Cook material parameters from machining simulations. Advanced Materials Research, vol. 223, 2011, p. 277-285.

[SHU 09] Shuo Y., WANG X., Li W., XU B.

Numerical Investigation on Effects of Interactions Between Particles on Coating Formation in Cold Spraying. Journal of Thermal Spray Technology, vol. 18, 2009, p. 686-693.

[SMO 11] SMOJVER I., IVANČEVIĆ D.

Bird strike damage analysis in aircraft structures using Abaqus/Explicit and coupled Eulerian Lagrangian approach. Composites Science and Technology, vol. 71, $\mathrm{n}^{\mathrm{O}} 4$, 2011, p. 489-498.

[STE 99] Steenkiste T. V., Smith J., Teets R., Moleski J., Gorkiewicz D., Tison R., Marantz D., Kowalsky K., II W. R., Zajchowski P., Pilsner B., MCCUNE R., BARNETT K.

Kinetic spray coatings. Surface and Coatings Technology, vol. 111, n ${ }^{\circ} 1,1999$, p. 6271.

[STE 02] Steenkiste T. V., Smith J., Teets R.

Aluminum coatings via kinetic spray with relatively large powder particles. Surface and Coatings Technology, vol. 154, $\mathrm{n}^{\mathrm{O}} 2-3,2002$, p. 237-252.

[SUD 07] Sudharshan P. P., Srinivasa R. D., Joshi S., Sundararajan G. Effect of Process Parameters and Heat Treatments on Properties of Cold Sprayed Copper Coatings. Journal of Thermal Spray Technology, vol. 16, $\mathrm{n}^{\mathrm{O}}$ 3, 2007, p. 425-434.

[SUH 13] Suhonen T., Varis T., Dosta S., Torrell M., Guilemany J.

Residual stress development in cold sprayed $\mathrm{Al}, \mathrm{Cu}$ and Ti coatings. Acta Materialia, vol. 61, no 17, 2013, p. 6329-6337.

[SWE 94] Swegle J., Attaway S., Heinstein M., Mello F., Hicks D. An analysis of smoothed particle hydrodynamics. Mar 1994.

[TSU 97a] TSUi Y., Clyne T.

An analytical model for predicting residual stresses in progressively deposited coatings Part 1: Planar geometry. Thin Solid Films, vol. 306, n 1, 1997, p. 23-33. 
[TSU 97b] Tsui Y., Clyne T.

An analytical model for predicting residual stresses in progressively deposited coatings Part 2: Cylindrical geometry. Thin Solid Films, vol. 306, n ${ }^{0}$ 1, 1997, p. 34-51.

[TSU 97c] TSUi Y., Clyne T.

An analytical model for predicting residual stresses in progressively deposited coatings Part 3: Further development and applications. Thin Solid Films, vol. 306, ${ }^{\circ}$ 1, 1997, p. 52-61.

[VEN 11] Venkatesh L., Chavan N. M., Sundararajan G.

The Influence of Powder Particle Velocity and Microstructure on the Properties of Cold Sprayed Copper Coatings. Journal of Thermal Spray Technology, vol. 20, n 5, 2011, p. 1009-1021.

[WAN 08] WANG Y., YANG Z.

Finite element model of erosive wear on ductile and brittle materials. Wear, vol. 265, $n^{0}$ 5-6, 2008, p. 871-878.

[WOY 99] Woytowitz P., Richman R.

Modeling of damage from multiple impacts by spherical particles. Wear, vol. 233235, 1999, p. 120-133.

[WU 01] WU C.

Finite Element Analysis of Particle Impact Problems. PhD Thesis, Aston University, Birmingham, UK, 2001.

[XIO 08] Xiong Y., Kang K., Bae G., Yoon S., Lee C.

Dynamic amorphization and recrystallization of metals in kinetic spray process. Applied Physics Letters, vol. 92, $\mathrm{n}^{\mathrm{o}}$ 19, 2008, Page 194101.

[YIL 11] Yildirim B., Muftu S., Gouldstone A.

Modeling of high velocity impact of spherical particles. Wear, vol. 270, $\mathrm{n}^{0}$ 9-10, 2011, p. 703-713.

[YIN 10] YIN S., WANG X., XU B., LI W.

Examination on the Calculation Method for Modeling the Multi-Particle Impact Process in Cold Spraying. Journal of Thermal Spray Technology, vol. 19, 2010, p. 10321041

[YIN 11] Yin S., WANG X., Li W., JiE H.

Effect of substrate hardness on the deformation behavior of subsequently incident particles in cold spraying. Applied Surface Science, vol. 257, $\mathrm{n}^{\mathrm{O}}$ 17, 2011, p. 7560-7565.

[ZAH 06] Zahiri S. H., Fraser D., Gulizia S., JAHEdi M.

Effect of processing conditions on porosity formation in cold gas dynamic spraying of copper. Journal of Thermal Spray Technology, vol. 15, $\mathrm{n}^{\mathrm{O}}$ 3, 2006, p. 422-430.

[ZAH 09] ZAHIRI S. H., ANTONIO C. I., JAHEDi M.

Elimination of porosity in directly fabricated titanium via cold gas dynamic spraying. Journal of Materials Processing Technology, vol. 209, nº 2, 2009, p. 922-929. 
NOM: XIE

DATE de SOUTENANCE: Le 27 Mai 2014

Prénoms: Jing

TITRE: SIMULATION OF COLD SPRAY PARTICLE DEPOSITION PROCESS

NATURE: Doctorat

École doctorale: MEGA

Spécialité: Mécanique - Génie Mécanique - Génie Civil

RÉSUMÉ:

Cold spray is a rapidly developing coating technology for depositing materials in the solid state. The cold spray particle deposition process was simulated by modeling the high velocity impacts of spherical particles onto a flat substrate under various conditions. We, for the first time, proposed the Couple Eulerian Lagrangian (CEL) numerical approach to solve the high strain rate deformation problem. The capability of the CEL numerical approach in modeling the Cold Spray deposition process was verified through a systematic parameter study, including impact velocity, initial particle temperature, friction coefficient and materials combination. The simulation results by using the CEL numerical approach agree with the experimental results published in the literature. Comparing with other numerical approaches, which are Lagrangian, ALE and SPH, the CEL analyses are generally more accurate and more robust in higher deformation regimes. Besides simulating the single particle impact problem, we also extended our study into the simulation of multiple impacts. A FCC-like particles arrangement model that inspired by the crystal structure was built to investigate the porosity rate and residual stress of deposited particles under various conditions. We observed not only the 3D profiles of voids, but also their distributions and developments during different procedures. Higher impact velocity and higher initial temperature of particles are both of benefit to produce a denser cold spray coating. The compressive residual stresses existed in the interface between the particle and substrate is mainly caused by the large and fast plastic deformation. Another simplified model for multiple impacts was created for the simulation of surface erosion. A severe surface erosion is the result of a high impact velocity, a high friction coefficient and a low contact angle. Two element failure models suitable for high-strain-rate dynamic problems were introduced in this study. One is shear failure model driven by Johnson-Cook plastic yielding; another one is called tensile failure model, which the material fails when the mean stress reaches the ultimate tensile strength. For a ductile material as Copper, it followed two fracture modes in our study, which are tensile failure mode and shear failure mode. The former one mainly occurred beneath the substrate surface and the periphery of substrate craters, nevertheless the latter one was found predominately at the surface of craters. Four steps were found during the propagation of crack: void formation; crack formation; crack growth; coalescence and failure. A simple criterion equation was derived based on the simulation results for predicting the initiation of damage, which the erosion velocity $v_{\text {ero }}$ is a function of contact angle $\theta$ and erosion velocity for normal impact $v_{\pi / 2}$. The equivalent plastic strain could also be a parameter for identifying the onset of damage, identified as being 1.042 for Copper in our study.

MOTS-CLÉS: Cold Spray, CEL, multiple impacts, Porosity rate, Residual stress, Erosion rate, Fracture

Laboratoire(s) de recherche: Laboratoire de Mécanique des Contacts et des Structures

UMR CNRS 5259 - INSA de Lyon

18-20 rue des Sciences

69621 Villeurbanne Cedex FRANCE

Directeur de thèse: Monsieur le Professeur Daniel NÉLIAS

Président du jury: le président

Composition du jury: Vincent GUIPONT Ghislain MONTAVON Bernard NORMAND Kazuhiro OGAWA Yuji ICHIKAWA Stéphane KNITTEL
Philippe BERTRAND

Daniel NÉLIAS

Vincent ROBIN
Alain COMBESCURE

Hélène WALTER-LE BERRE 